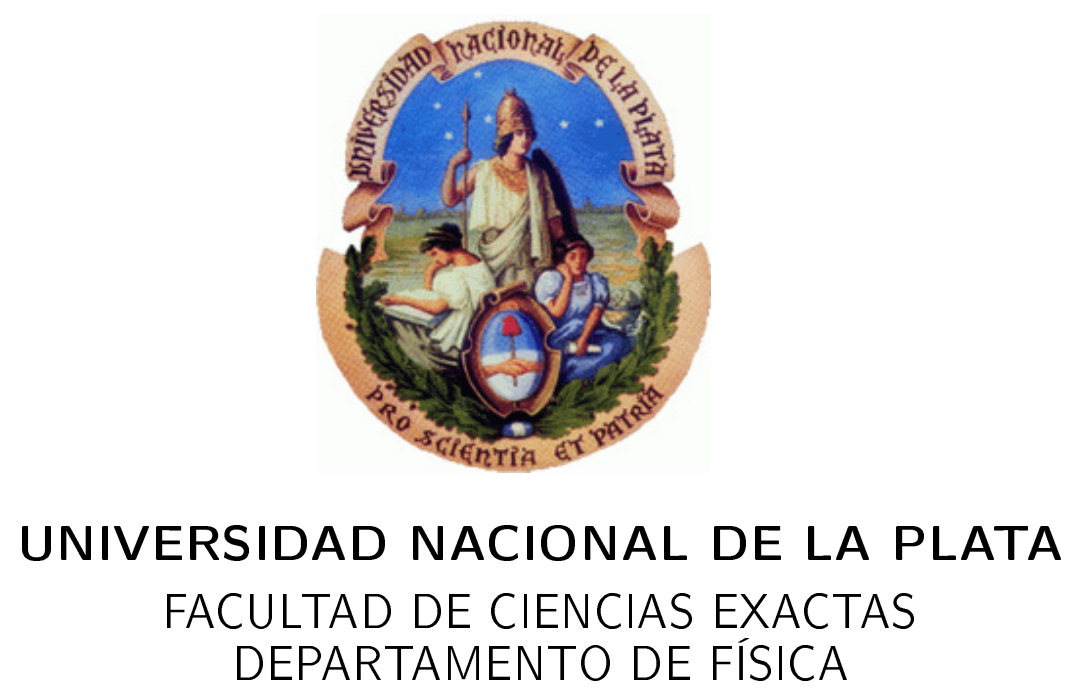

Tesis presentada para optar al grado de Doctor de la Facultad de Ciencias Exactas

\title{
Dinámica fuera de equilibrio de sistemas cuánticos aislados
}

Lic. Emilio Nicolas Nessi

Director: Dr. Carlos Aníbal lucci

Año: 2015 

No importa qué tan profundo sea nuestro conocimiento de las ideas abstrusas, es como un cabello en la inmensidad del vacío. Por muy grande que sea nuestra experiencia en las cosas de este mundo, es como una gota votada en un abismo insondable.

- Tokusan. 



\section{Agradecimientos}

Los años de vida son una cosa preciosa. El alma humana liba el néctar de la experiencia a cada segundo, a través de cada fenómeno que se posa frente al ojo de la conciencia, sin importar si lo consideramos importante o no. La suma de la experiencia vital de una persona es un grueso entramado de experiencias e interacciones que van perfeccionando al alma, le van dando sabiduría, van despertando el amor en ella. Cada persona que nos cruzamos es, en ese instante, la persona más importante del mundo para nosotros, cada cosa que hacemos es, en ese instante, la cosa más importante del mundo, no puede ser de otra manera. Han pasado ya más de doce años desde que empecé mis estudios universitarios, y casi cinco desde que comencé con los estudios de posgrado. Fueron años muy intensos y he acumulado mucho néctar desde diferentes fuentes. Es virtualmente imposible agradecer a todos los que merecen el agradecimiento por todo lo que han hecho por mí, ya que incluso a veces lo han hecho inconcientemente, sin darse cuenta. Vaya entonces un agradecimiento general, desde el fondo del corazón, a todos.

En una vena un poco menos universal, quiero agradecer a mi director Aníbal Iucci por todos estos años de trabajo codo a codo tratando de entender los problemas que estudiábamos y de comunicar nuestro entendimiento a la comunidad científica (una tarea no menos ardua que la primera). Quiero agradecer a Carlos Naón por haberme abierto las puertas de su grupo. A mis compañeros de oficina por los innumerables favores (grandes y pequeños) que me han facilitado sin nunca buscar nada a cambio.

Quiero agradecer a mis padres por haberme acompañado y dado soporte a mis iniciativas durante tantos años.

Por último, quiero agradecer a Micaela, mi compañera del Camino, por ser el tutor recto sin el que esta pequeña planta hubiera crecido torcida. 



\section{Resumen}

En esta tesis se estudia la dinámica de no equilibrio de sistemas cuánticos aislados de muchos cuerpos. El protocolo de no equilibrio estudiado es el de un quench cuántico, es decir, una perturbación abrupta al sistema que se produce al variar instantáneamente uno de los parámetros del Hamiltoniano. Estamos particularmente interesados en las escalas de tiempo y en los mecanismos de relajación de los sistemas cuánticos aislados. Para atacar este problema utilizamos una variedad de técnicas analíticas para indagar diversos modelos. En el Capítulo 2 utilizamos la bosonización en una dimensión para estudiar la relajación por dephasing (el mecanismo que domina a tiempos cortos) en un sistema de fermiones unidimensionales con interacciones de corto y largo alcance. En el Capítulo 3 investigamos la formación de estados metaestables de tiempos cortos (estados pretermalizados) como fruto de la evolución por dephasing en un sistema de fermiones en dos dimensiones usando bosonización multidimensional. En el Capítulo 4 deducimos ecuaciones de movimiento para funciones de correlación de pocos puntos en sistemas débilmente interactuantes utilizando el formalismo del operador proyección. En el Capítulo 5 utilizamos estas ecuaciones para analizar la dinámica de tiempos largos de un sistema unidimensional de fermiones cerca del punto integrable $X X Z$. En este Capítulo también estudiamos la dinámica de un sistema unidimensional de bosones usando la aproximación truncada de Wigner (TWA por sus siglas en inglés), una aproximación válida en el límite semiclásico. Los hallazgos de nuestro trabajo pueden enunciarse de manera suscinta: cuando los canales de relajación del sistema son escasos y para energías lo suficientemente bajas emergen estados metaestables entre el estado inicial y el estado final de la evolución. Éstos pueden tener vidas medias muy grandes y obturar completamente la observación del equilibrio térmico en simulaciones numéricas o en experimentos. Los estados metaestables surgen como resultado del dephasing entre ciertos modos cuasilibres del sistema. Además, tales estados estacionarios admiten una descripción estadística en términos de un ensamble de Gibbs generalizado que toma en cuenta todas las cantidades conservadas del modelo efectivo de tiempos cortos. A tiempos mayores las colisiones inelásticas llevan al equilibrio térmico final. En el Capítulo 6 se detallan las conclusiones de nuestro trabajo y diversas perspectivas abiertas. 



\section{Índice general}

Agradecimientos

Resumen III

Introducción 1

1. Termalización y dinámica en sistemas cuánticos aislados 7

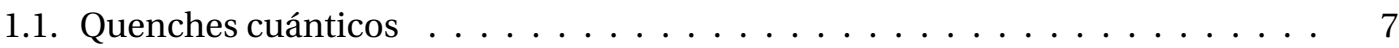

1.2. Termalización cuántica: Ensambles canónicos y GGE . . . . . . . . . . . . . . . . 8

1.3. Hipótesis de Autoestados Termalizados . . . . . . . . . . . . . . . . . . . . 12

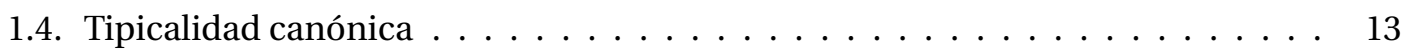

1.5. Dinámica de no equilibrio . . . . . . . . . . . . . . . . . . . . . 14

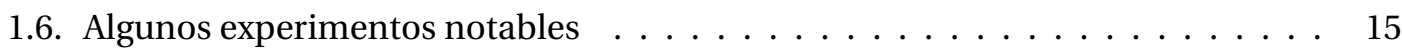

2. Dephasing y el ensamble de Gibbs generalizado 19

2.1. El modelo de Luttinger y bosonización . . . . . . . . . . . . . . . . . . 20

2.2. Quenches en la interacción y dephasing . . . . . . . . . . . . . 23

2.3. Ensamble generalizado de Gibbs . . . . . . . . . . . . . . . . . . . . 27

2.4. Relevancia del ML para la dinámica de modelos microscópicos . . . . . . . . . . 37

3. Pretermalización $\quad 43$

3.1. Análisis perturbativo de un quench de interacción en un gas de Fermi . . . . . 44

3.2. Líquidos de Fermi en equilibrio y bosonización . . . . . . . . . . . . . . . . 46

3.3. Quenches en la interacción y bosonización . . . . . . . . . . . . . . . 52

3.4. Cálculo de las funciones de correlación después de un quench utilizando boso-

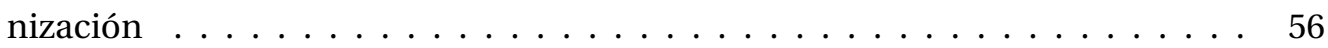

3.4.1. Resolviendo la dinámica . . . . . . . . . . . . . . . . . . . 57

3.4.2. Función de Green fermiónica . . . . . . . . . . . . . . . . . . . . . 59

3.4.3. Estado estacionario . . . . . . . . . . . . . . . . . . . 63

3.4.4. Ensamble de Gibbs generalizado . . . . . . . . . . . . . . . . . 65

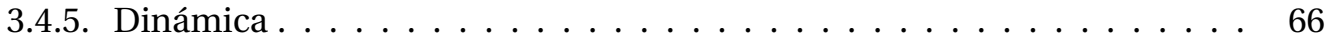

3.5. Otras perspectivas teóricas sobre la pretermalización y evidencias experimentales 68 
4. Proyección dinámica y ecuaciones de movimiento fuera de equilibrio 73

4.1. Modelo y quench . . . . . . . . . . . . . . . . 76

4.2. Técnica del operador proyección y ecuaciones de movimiento . . . . . . . . 76

4.3. Aproximaciones adicionales . . . . . . . . . . . . . . . 82

4.3.1. Aproximación de tiempos cortos . . . . . . . . . . . . . . 82

4.3.2. Ecuación de Boltzmann . . . . . . . . . . . . . . . . 82

4.3.3. Aproximaciones y mecanismos de relajación . . . . . . . . . . . . . . . . . . . . . . . . . . . . .

4.4. Funciones de correlación de orden más alto . . . . . . . . . . . . . . 83

5. Estados pretermalizados y comportamiento tipo vidrio en sistemas casi integrables $\begin{array}{ll}\text { en una dimensión } & 89\end{array}$

5.1. Fermiones débilmente interactuantes . . . . . . . . . . . . . . . . . 91

5.1.1. Modelo . . . . . . . . . . . . . . . . . 91

5.1.2. Dinámica en el límite termodinámico . . . . . . . . . . . . . . . . 93

5.1.3. Dinámica de sistemas finitos y escalas de tiempo . . . . . . . . . . . . 96

5.1.4. Discusión . . . . . . . . . . . . . . . . . . 100

5.2. Bosones fuertemente interactuantes: Estados pretermalizados y heterogeneidad

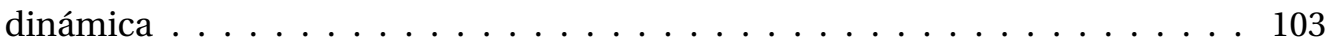

5.2.1. El caso particular del modelo de Bose-Hubbard . . . . . . . . . . . . 103

5.2.2. Dinámica cuántica: Resultados de la TWA . . . . . . . . . . . . . 104

5.2.3. Propiedades de las soluciones clásicas . . . . . . . . . . . . . 110

5.3. Estados pretermalizados lejos de un punto integrable . . . . . . . . . . . . 121

$\begin{array}{lr}\text { 6. Conclusiones y perspectivas } & 123\end{array}$

A. Apéndice $\quad 125$

A.1. Propiedades del operador proyección $P(t) \ldots \ldots \ldots \ldots$. . . . . . . . 125

A.2. Exponenciales ordenadas . . . . . . . . . . . . . . . . . 127

A.2.1. Representación de interacción . . . . . . . . . . . . . . . . 127

A.2.2. Representación de Heisenberg . . . . . . . . . . . . . . . . 128

$\begin{array}{lr}\text { Bibliografía } & 140\end{array}$ 


\section{Introducción}

Nuestra experiencia cotidiana está conformada, en gran medida, por fenómenos fuera de equilibrio. El mismísimo concepto de la vida está relacionado intrínsecamente con el de estructura disipativa, es decir, un sistema abierto que opera fuera del equilibrio termodinámico y que presenta alguna especie de estado estacionario con un alto grado de ordenamiento de los componentes [116]. El no equilibrio está ligado a la diversidad, mientras que el equilibrio a la uniformidad. De hecho, son innumerables las maneras de sacar a un sistema fuera de equilibrio, pero, dados los valores de las cantidades conservadas globales de un sistema (como la energía y el número de partículas), hay sólo un estado compatible con el equilibrio termodinámico.

Existe un hecho que es tan fascinante como la diversidad del no equilibrio y es la expectación de que un sistema, luego de haber sido perturbado (mucho o poco) fuera de equilibrio, debe llegar, al cabo de una evolución autónoma (aislado del entorno) a un estado compatible con el equilibrio térmico. De hecho, muchas definiciones operativas de equilibrio térmico se basan en esta conjetura, como por ejemplo, la presentada en el libro de Ruelle [128]:

«Los estados de equilibrio pueden definirse operativamente. El estado de un sistema aislado tiende a un estado de equilibrio cuando el tiempo tiende a $+\infty$ ».

La variedad del no equilibrio debe colapsar en la uniformidad del equilibrio entonces. ¿Cómo es esto posible? $i$ Hay alguna clase de universalidad en ese proceso o es necesario analizar caso por caso? ¿Cuáles son los mecanismos que gobiernan su dinámica? Estas preguntas han motivado el trabajo de muchos físicos por generaciones, entre los cuales el más notable es, quizás, Ludwig Boltzmann. Además, estos interrogantes, irresueltos muchos de ellos, custionan las bases mismas de la Mecánica Estadística, la cual brinda una potente descripción de los sistemas en equilibrio pero no explica cómo emerge este estado a partir de una situación genérica [128].

En esta tesis buscamos hacer una contribución a esta área de la física. Para ello nos centraremos en la dinámica de no equilibrio de sistemas aislados en regímenes en que los efectos cuánticos no pueden despreciarse.

Señalemos que para sacar a un sistema aislado del equilibrio debemos romper su aislación 
con el entorno. Razonemos así: el sistema fue preparado en un cierto estado inicial a través de un proceso que ignoramos. Supongamos que se encuentra en equilibrio (por ejemplo, en un estado estacionario de un cierto Hamiltoniano). Si dejamos al sistema aislado en su estado de equilibrio, éste permanecerá en ese estado por toda la eternidad. Para sacarlo de equilibrio debemos ejercer trabajo sobre él, debemos inyectarle una cierta cantidad de energía. Aquí es donde entra el protocolo de no equilibrio, el cual puede incluir cualquier tipo de acción ejercida sobre el sistema para sacarlo de su letargo inicial. Nos concentraremos en protocolos de no equilibrio que puedan modelarse a través de una dependencia temporal en los parámetros del Hamiltoniano del sistema. En especial, consideraremos el caso en que el Hamiltoniano cambia abruptamente en un cierto instante de tiempo. Esto definie un Hamiltoniano inicial, que nos posibilita preparar al sistema en un cierto estado estacionario inicial, y un Hamiltoniano de evolución. Para observar equilibración la etapa en la que el Hamiltoniano varía con el tiempo, el cambio abrupto, debe tener una extensión temporal finita y pequeña. Es decir, en algún momento debemos dejar de bombear energía en el sistema y dejarlo evolucionar por su cuenta. Este escenario fue bautizado como «quench cuántico» en la literatura y es el protocolo de no equilibrio más simple que podamos imaginar.

A pesar de ser un problema histórico, el estudio de la dinámica de no equilibrio de sistema cuánticos aislados ha vuelto a ganar atención debido al desarrollo de tecnologías que permiten recrear en el laboratorio esta situación de no equilibrio, en la cual el sistema bajo estudio permanece aislado del entorno por períodos de tiempo prolongados permitiendo observar su evolución y relajación. Esto se logra manipulando agregados de átomos (cuyo número puede variar desde unos pocos hasta una cantidad macroscópica) a una temperatura del orden del $n K$ para la cual los efectos cuánticos son importantes [11]. Su contenedor está constituido por el campo electromagnético estacionario generado por un cierto arreglo de lásers o imanes. Además, estos lasers pueden crear una red óptica, introduciendo un potencial periódico para el movimiento atómico. La capacidad de variar la geometría de este arreglo utilizando herramientas ópticas de precisión, en conjunción con el hecho de que los átomos (por más que sean neutros eléctricamente) interactúan con ese campo, permite escencialmente diseñar el Hamiltoniano del sistema a medida, y lo que es muy importante para el problema que pretendemos estudiar, modificarlo en tiempo real, algo que era prácticamente imposible en los sistemas tradicionales de materia condensada. El experimento de Kinoshita et al. [76], en el cual se observó que un gas unidimensional de bosones preparado en una condición inicial de no equilibrio no alcanzaba el equilirbio térmico (ni ningún tipo de estado estacionario) en las escalas de tiempo experimentales, despertó un gran interés en la comunidad y reavivó la temática en cuestión.

En general, se espera que un sistema en el límite termodinámico alcance el estado de equilibrio térmico luego de un quench. Es decir, que alcance un estado que pueda describirse, bajo ciertas condiciones (volveremos sobre esto con todo detalle en el desarrollo de la tesis), por una matriz densidad que represente alguno de los ensambles canónicos de la Mecánica Estadística. Por ejemplo, si $\rho(t)$ es la matriz densidad del sistema en el instante de tiempo $t$, esperamos que $\rho(t \rightarrow \infty) \propto e^{-\beta H}$, en donde $\beta$, la inversa de la temperatura, queda fijada 
por la energía del sistema (la cual se conserva luego del quench debido a la evolución unitaria). Sin embargo, para sistemas integrables, aquellos que poseen un número extensivo de cantidades conservadas, debemos revisar tal expectación. De hecho, en el aporte seminal de la Ref. [124] fue propuesto que un sistema integrable debe relajar luego de un quench a un estado compatible con una matriz densidad que tenga en cuenta todas las cantidades conservadas del modelo, y no sólo la energía o el número de partículas. En símbolos, si $I_{\alpha}$ son las cantidades conservadas del sistema, se espera que éste relaje a un estado que quede bien descripto por una matriz densidad $\rho(t \rightarrow \infty) \propto e^{-\sum_{\alpha} \lambda_{\alpha} I_{\alpha}}$, en donde $\lambda_{\alpha}$ son multiplicadores de Lagrange que quedan fijados por el valor de expectación de las cantidades conservadas en el estado inicial (más sobre esto en el capítulo 1). Tales ensambles son llamados ensambles de Gibbs generalizados (GGE). La validez del GGE ha sido demostrada para una variedad de sistemas integrables [17, 3, 18, 30, 67, 44, 108, 98]. En cuanto a la transición integrabilidad-no integrabilidad, hay resultados recientes que apuntan al hecho de que una ruptura infinitesimal de la integrabilidad es suficiente para asegurar, en el límite termodinámico, que las características del estado asintótico del sistema sean térmicas [122].

Respecto a la dinámica, existen varios canales de relajación que posibilitan alcanzar el equilibrio térmico. A tiempos cortos domina el llamado dephasing o desfasaje (el que estudiaremos en detalle en el Capítulo 2), un mecanismo genérico que no depende de los detalles de las interacciones entre las partículas del sistema $[8,57]$. De hecho, este mecanismo está presente también para sistemas sin interacciones, en cuyo caso es el único posible y es análogo a la expansión balística incial en fluidos clásicos. El dephasing no es suficiente para llevar al sistema al equilibrio térmico, es decir, para borrar completamente los detalles sobre las condiciones iniciales. Para ello es necesario que existan otros canales de relajación, tales como colisiones entre las partículas (como las que son modeladas mediante una ecuación de Boltzmann). Sin embargo, hay casos en los que estos canales de relajación están altamente suprimidos, por ejemplo, en las cercanías de un punto integrable en sistemas unidimensionales (este será el tema del Capítulo 5). En tales casos el sistema indefectiblemente quedará atrapado en estados metaestables no térmicos que sólo han perdido parte de la memoria de los detalles de las condiciones iniciales gracias al dephasing. En otras palabras, existirá una clara separación entre las escalas de tiempo en las que domina el dephasing y los demás canales de relajación. La probabilidad de ocurrencia de eventos de relajación diferentes del dephasing (como colisiones con transferencia no nula de momento) representará, entonces, una medida de la vida media de tales estados metaestables. Tales estados que surgen a tiempos cortos como resultado de la dinámica de dephasing son designados como «estados pretermalizados» en la literatura $[95,40,78]$. Haciendo un poco de etimología podemos señalar que el concepto de la pretermalización surgió en el contexto de la dinámica de no equilibrio de modelos de la física de altas energías, los cuales son útiles para describir las primeras etapas de evolución del universo primigenio y las colisiones entre iones pesados en experimentos de aceleradores tales como el RHIC [6]. En tales sistemas se descubrió que ciertas cantidades pueden alcanzar su valor térmico en etapas tempranas de la evolución luego de un quench como fruto únicamente del dephasing. Es decir, el dephasing es suficiente para borrar totalmente los detalles de la 
condición inicial al nivel de ciertos observables, entre los cuales el ejemplo más prominente es la energía cinética del sistema [8]. Se dice que tales cantidades pretermalizan. En el marco del estudio de la dinámica de no equilibrio de sistemas más propios de materia condensada (un líquido de Fermi, ejemplo sobre el que discutiremos extensivamente en el Capítulo 3) [95] se encontró el mismo fenómeno, pero con la novedad de que éste estaba asociado con la emergencia de tales estados metaestables que pasaron a llamarse, por extensión, estados pretermalizados.

Se ha visto que en sistemas débilmente no integrables tales estados metaestables pueden describirse en términos de un GGE, es decir, utilizando una matriz densidad que contenga la información acerca de ciertas cantidades conservadas efectivas del modelo en cuestión [78]. La estrecha relación que liga el dephasing, el GGE y la pretermalización es uno de los puntos neurálgicos de esta tesis, al que están dedicados los Capítulos 2 y 3 . Básicamente, pudimos corroborar que en los sistemas estudiados la dinámica de dephasing a tiempos cortos puede capturarse a través de un modelo efectivo integrable, por más que el sistema sea no integrable. El GGE que describe el estado pretermalizado está entonces naturalmente relacionado con tal modelo efectivo. En capítulos siguientes también argumentaremos a favor de la generalidad de esta conclusión.

El otro punto principal de esta tesis es determinar las escalas de tiempo de la relajación de un sistema aislado. En particular, hemos investigado cuáles son las variables relevantes que determinan la vida media de los estados pretermalizados y para qué regiones de parámetros éstos están ausentes (relajación en un solo paso). El resultado de tal investigación está presentado en el Capítulo 5. Nuestros resultados revelan que en regiones de parámetros en las cuales los canales de relajación se encuentran restringidos (en las cercanías de un punto integrable en sistemas unidimensionales por ejemplo) y cuando el quench es lo suficientemente débil (la energía del estado inicial es cercana a la energía del estado fundamental del Hamiltoniano que gobierna la evolución) tales estados metaestables pueden tener una vida media muy grande, lo cual puede hacer prácticamente imposible la observación del estado de equilibrio térmico en los experimentos.

Desde el punto de vista técnico, la dinámica de no equilibrio de un sistema cuántico aislado de muchos cuerpos plantea un desafío tremendo. Por ejemplo, los métodos numéricos estándar están confinados a tiempos cortos y sistemas grandes o a sistemas pequeños y tiempos arbitrarios, como por ejemplo el método del grupo de renormalización de la matriz densidad dependiente del tiempo (t-DMRG por sus siglas en inglés) o diagonalización exacta, respectivamente. Los métodos analíticos usuales (tales como el formalismo de Keldysh) también poseen muchas limitaciones. En esta tesis hemos usado una variedad de técnicas no convencionales para atacar el problema del no equilibrio. Para obtener los resultados presentados en los primeros capítulos de esta tesis, utilizamos la técnica de la bosonización, tanto en $D=1$ como en dimensión superior. Esta técnica es uno de los pilares para atacar sistemas unidimensionales en equilibrio [51], aunque también ha sido extendida para poder describir sistemas en dimensión superior [65]. En el transcurso de las investigaciones que 
son reportadas en esta tesis extendimos su validez más allá de los límites del equilibrio, de manera de poder capturar la dinámica de tiempos cortos luego de un quench débil. Para acceder a escalas de tiempos mayores fue necesario explorar otras técnicas. El Capítulo 4 está especialmente consagrado a presentar los resultados de esta exploración. En él se deduce un sistema de ecuaciones de evolución para funciones de correlación de pocos puntos que describen la dinámica de sistemas débilmente interactuantes luego de un quench. Estas ecuaciones fueron deducidas utilizando el formalismo del operador proyección [54, 12, 126], una técnica muy conocida en el campo de la física estadística de no equilibrio.

Hemos organizado la exposición de la siguiente manera: en el Capítulo 1, para poner el trabajo en contexto, presentamos algunas generalidades sobre la termalización cuántica y comentamos los experimentos más relevantes en este campo. Las contribuciones originales de la tesis se desarrollan a partir del Capítulo 2, en el cual analizamos en detalle uno de los mecanismos más importantes de relajación en sistemas cuánticos aislados, el dephasing entre modos cuasilibres, el cual domina a tiempos cortos. En el Capítulo 3 abordamos el tema de la emergencia de estados metaestables no térmicos que se interponen entre el estado inicial y el estado asintótico del sistema, los cuales surgen como resultado de la dinámica de dephasing (estados pretermalizados). En el Capítulo 4 presentamos la técnica del operador proyección la cual nos servirá luego para deducir ecuaciones que gobiernan la dinámica de las funciones de correlación de pocos puntos con las que podremos acceder a la dinámica de tiempos largos. En el Capítulo 5 analizamos en dos sistemas unidimensionales las variables más relevantes que determinan la vida media de los mencionados estados pretermalizados, y por lo tanto, modifican las escalas de tiempos para la termalización. En uno de los casos utiliszamos las ecuaciones de movimiento deducidas en el capítulo precedente y en el otro la aproximación truncada de Wigner, una técnica semiclásica. En este capítulo, además, describimos paralelos fenomenológicos entre la física de la pretermalización en sistemas cuánticos aislados y la de los sistemas vidriosos.

El contenido original de esta tesis doctoral está publicado (o aceptado/enviado para publicar) en los siguientes artículos:

- "Quantum Quench Dynamics of the Coulomb Luttinger Model". Emilio Nicolas Nessi y Aníbal Iucci. Phys. Rev. B 87, 085137 (2013).

- "Quantum Quench and Prethermalization Dynamics in A Two-Dimensional Fermi Gas with Long-range Interactions ”. Emilio Nicolas Nessi, Aníbal Iucci y Miguel Cazalilla. Phys. Rev. Lett. 113, 210402 (2014).

- "Equations of Motion for the Out-of-Equilibrium Dynamics of Isolated Quantum Systems from the Projection Operator Technique". Emilio Nicolas Nessi y Aníbal Iucci. Jour. Phys.: Conf. Ser. 568, 012013 (2014). Proceedings of 27th Conference on Low Temperature Physics.

- "Prethermalization and glassiness in the bosonic Hubbard model". Ignacio Salazar 


\section{Índice general}

Landea y Emilio Nicolas Nessi. Phys. Rev. A. 91, 063601 (2015).

- "Glass-like behavior in a system of one dimensional fermions after a quantum quench". Emilio Nicolas Nessi y Aníbal Iucci. Preprint: arXiv: 1503.02507 (2015, enviado a Physical Review B). 


\section{Termalización y dinámica en sistemas cuánticos aislados}

\subsection{Quenches cuánticos}

El problema general que nos estamos planteando es la evolución fuera de equilibrio de un sistema cuántico aislado. De todos los protocolos de no equilibrio imaginables, a lo largo de esta tesis consideraremos el de una perturbación abrupta, un quench cuántico. Para realizar un quench cuántico necesitamos dos ingredientes, un estado inicial con matriz densidad $\rho(t=0)$ y un Hamiltoniano de evolución $H$. La condición de no equilibrio es $[H, \rho(0)] \neq 0$. Nos interesa indagar sobre el estado del sistema a tiempos finitos. En la representación de Schrödinger es la matriz densidad que evoluciona según la ecuación de Liouville-Von Neumann $(\hbar=1)$ :

$$
i \frac{d \rho(t)}{d t}=[H, \rho(t)]
$$

lo que implica que $\rho(t)=e^{-i H t} \rho(0) e^{i H t}$. En este tipo de protocolos de no equilibrio se inyecta una cantidad extensiva de energía en el sistema. De hecho, supongamos que el estado inicial del sistema es el estado fundamental $\left|\Psi_{0}\right\rangle\left(\rho(0)=\left|\Psi_{0}\right\rangle\left\langle\Psi_{0}\right|\right)$ de un cierto Hamiltoniano $H_{0}$. En el contexto de los experimentos realizados con átomos fríos, $H_{0}$ correspondería a una cierta configuración de los lásers que conforman la red óptica. Preparar el estado $\left|\Psi_{0}\right\rangle$ correspondería a enfriar adiabáticamente (idealmente hasta el cero absoluto) la muestra de materia en presencia de la configuración de lásers que determinan $H_{0}$. Una vez que hemos enfriado la muestra podemos desconectar todo contacto con el exterior. Si realmente hemos preparado el estado $\left|\Psi_{0}\right\rangle$ no habrá evolución en los observables en tiempos subsiguientes dado que el estado del sistema será un estado estacionario del Hamiltoniano $H_{0}$ determinado por la configuración de lásers. Para realizar el quench debemos modificar abruptamente (en comparación con las escalas de tiempo propias de la muestra de materia que estemos considerando) la configuración de los lásers para que pasen a simular el Hamiltoniano $H$. Para ello debemos ejercer un cierto trabajo sobre el sistema, es decir, debemos romper, por un instante, la aislación perfecta. Una vez que la configuración de lásers de $H$ está fija dejamos evolucionar el sistema. Entonces, como se dijo, para realizar el quench cuántico fue necesario 
ejercer un trabajo extensivo (dado que modificamos la configuración de lásers en toda la extensión del sistema).

Dentro de los quenches cuánticos, el escenario que más hemos estudiado es el de un quench de interacción, es decir, la situación en la que el estado inicial es un estado estacionario (autoestado o estado térmico por ejemplo) de un Hamiltoniano no interactuante, mientras que el Hamiltoniano de evolución involucra interacciones entre los componentes. Este escenario puede realizarse en experimentos con átomos fríos por medio de las llamadas resonancias de Feschbach [11] o manipulando la profundidad de la red óptica en algunos casos [55]. Cabe mencionar que el quench de interacción es un protocolo de no equilibrio ilustre. De hecho, puede demostrarse que la hipótesis de caos molecular invocada por Boltzmann para deducir su ecuación es equivalente a que inicialmente las partículas estén completamente descorrelacionadas (sean partículas libres) [143], lo que implica que la ecuación de Boltzmann describe un quench de interacción clásico.

\subsection{Termalización cuántica: Ensambles canónicos y GGE}

El concepto clave detrás de la termalización en sistemas clásicos aislados es la ergodicidad, es decir, el hecho de que el promedio temporal de un cierto observable sea igual al promedio sobre todas las configuraciones que tengan la misma energía que el sistema que evoluciona. En otras palabras, que el promedio temporal del observable sea igual al promedio microcanónico. Para generalizar el concepto de termalización a sistemas cuánticos no es posible enfocarnos en la evolución del estado cuántico propiamente dicho, dado que este nunca relajará. Por ejemplo, en la base de autoestados de $H$, los elementos de matriz de la matriz densidad a tiempo $t$ son

$$
\rho_{n m}(t)=\left\langle\Psi_{n}|\rho(t)| \Psi_{m}\right\rangle=e^{-i\left(E_{n}-E_{m}\right) t} \rho_{n m}(0)
$$

en donde $\left|\Psi_{\alpha}\right\rangle$ son los autoestados del Hamiltoniano de evolución $H$ con autovalor $E_{\alpha}$. En particular, si consideramos un estado inicial puro $\operatorname{Tr}\left[\rho(0)^{2}\right]=1$, dado que la evolución unitaria conserva la traza, éste permanecerá puro para todo tiempo posterior y nunca podrá dar lugar en un estado mezcla con $\operatorname{Tr}\left[\rho^{2}\right]<1$, como se espera para un estado térmico. Tampoco aporta información analizar el comportamiento de los observables, debido a que en general es posible que estos no alcancen ningún estado estacionario, sea éste térmico o no. De hecho, consideremos la evolución de los operadores hermíticos

$$
O_{m, n}=\left|\Psi_{m}\right\rangle\left\langle\Psi_{n}|+| \Psi_{n}\right\rangle\left\langle\Psi_{m}\right|,
$$

Su valor de expectación dependiente del tiempo,

$$
\left\langle\psi(t)\left|O_{m, n}\right| \psi(t)\right\rangle=\left\langle\psi(0) \mid \Psi_{m}\right\rangle\left\langle\Psi_{n} \mid \psi(0)\right\rangle e^{i\left(E_{m}-E_{n}\right) t}+\text { h.c. },
$$


en donde $|\psi(t)\rangle=e^{-i H t}|\psi(0)\rangle$ es la función de onda dependiente del tiempo ( $|\psi(0)\rangle$ es el estado puro inicial), es oscilante y nunca alcanza un estado estacionario.

Detrás de estos ejemplos que nos llevan a considerar cuidadosamente la definición de termalización cuántica se encuentra el hecho de que la evolución unitaria de un sistema cuántico aislado conserva la información. De hecho, si $\rho(t)$ es la solución de la ecuación de Liouville Ec. (4.4), es fácil ver que la entropía de Von Neumann asociada $S(t)=\operatorname{Tr}[\rho(t) \log \rho(t)]$ es constante en el tiempo. Eso significa que la información acerca de la condición inicial nunca se pierde. Sin embargo, el hecho de que un sistema termalice está indisolublemente asociado a que debe alcanzar un estado que contenga información sólo acerca de unas ciertas cantidades conservadas globales del sistema, como la energía o el número de partículas. En otras palabras, el sistema debe alcanzar un estado que no guarde memoria de los detalles de la condición inicial.

La solución a esta paradoja está en focalizarnos en el estado de un cierto subsistema $A$ de nuestro sistema cuántico y en los observables locales $O$ que están deifnidos en él, y no en el estado global [4]. Esta observación también es lógica desde el punto de vista experimental, dado que las propiedades de un sistema pueden medirse experimentalmente sólo a través de medidas de observables locales. Con esto en mente, definamos primero relajación. Para ello, consideremos la matriz densidad de un subsistema $A$ (pequeño en relación al sistema total) con complemento $\bar{A}$, la cual puede calcularse a partir de la matriz densidad global $\rho$ como $\rho_{A}=\operatorname{Tr}_{\bar{A}}[\rho]$. Diremos que un sistema relaja si existe el límite del promedio temporal de la matriz densidad reducida de cualquier subsistema $A^{1}$, es decir, si existe una matriz densidad estacionaria $\rho_{\infty}$ tal que

$$
\lim _{T \rightarrow \infty} \frac{1}{T} \int_{0}^{T} d t \rho_{A}(t)=\operatorname{Tr}_{\bar{A}}\left[\rho_{\infty}\right] .
$$

Esta condición debe cumplirse para todo subsistema pequeño A. La Ec. (1.5) implica que el valor estacionario de cualquier observable local $O$ puede ser predecido por $\rho_{\infty}$ :

$$
\lim _{T \rightarrow \infty} \frac{1}{T} \int_{0}^{T} d t \operatorname{Tr}[O \rho(t)]=\operatorname{Tr}\left[O \rho_{\infty}\right],
$$

y lo mismo vale para cualquier función de correlación que involucre operadores en un subsistema acotado. Si bien estamos definiendo relajación en base a la existencia del límite del promedio temporal, para completar la definición, es natural requerir que las fluctuaciones alrededor de ese promedio sean pequeñas [144]. Por ejemplo, las fluctuaciones de valor de expectación propuesto en la Ec. (1.4) van a ser del mismo orden que el promedio mismo dado que hay una sola función oscilante. Sin embargo, notemos que éste es el valor de expectación de un observable altamente no local, construído en base a los autoestados del Hamiltoniano. Si la matriz densidad reducida relaja según la definición dada antes, podemos preguntarnos

\footnotetext{
${ }^{1}$ Esto se denomina relajación débil. También podría ocurrir una relajación fuerte, en la que la matriz densidad reducida relaja directamente sin necesidad de tomar el promedio temporal [4].
} 
acerca de sus propiedades. Diremos que el sistema termaliza si $\rho_{\infty}$ se corresponde con alguno de los ensambles canónicos de la mecánica estadística. Por ejemplo, que

$$
\rho_{\infty}=\frac{1}{Z} e^{-\beta_{e f} H},
$$

en donde la función de partición es $Z=\operatorname{Tr}\left[e^{-\beta_{e f} H}\right]$ y la temperatura efectiva $\beta_{e f}$ viene determinada por la relación $\operatorname{Tr}[H \rho(0)]=\frac{\operatorname{Tr}\left[H e^{-\beta_{e f} H}\right]}{\operatorname{Tr}\left[e^{-\beta_{e}{ }^{H}}\right]}$. Recordemos también que en el límite termodinámico todos los ensambles canónicos (microcanónico, canónico y gran canónico) proveen predicciones equivalentes.

En el marco de esta definición de termalización cuántica, la termalización del sistema cuántico aislado puede ser sólo aparente en el sentido de que a medida que consideramos subsistemas cada vez más grandes, esperamos que la matriz densidad reducida ya no relaje. Una pregunta interesante es si existe algún tamaño característico de subsistemas por encima de los cuales se pierde la relajación [10]. Dicho de otra manera, si fuéramos capaces de acceder a funciones de correlación de un número arbitrario de puntos deberíamos constatar que éstas se alejan de la forma térmica al aumentar su orden. La explicación intuitiva de esta perspectiva es que al considerar sólo observables y funciones de correlación locales, el resto del sistema actúa como un baño térmico para el subsistema que las contiene.

Sin embargo, la situación puede cambiar si el sistema es integrable. En dinámica clásica la definición de sistema integrable es precisa, se trata de un sistema con tantas cantidades conservadas en involución (cuyos corchetes de Poisson son nulos) como grados de libertad. En el dominio cuántico proveer una definición general de integrabilidad es todavía un problema abierto [28]. Sin embargo, según todos los criterios, un modelo cuántico integrable posee cantidades conservadas más allá de la energía o el número de partículas. Sin embargo, en esta tesis nos restringiremos a considerar el caso más simple de modelo integrable cuántico, los modelos cuadráticos (sin interacciones). En un modelo cuadrático $H=\sum_{q} \epsilon(q) \alpha_{q}^{\dagger} \alpha_{q}$, en donde $\epsilon(q)$ es la relación de dispersión y los operadores $\alpha(q)$ obedecen leyes de conmutación o anticonmutación canónicas, las cantidades conservadas, cuyo número viene dado por el tamaño del sistema, son los números de ocupación $\hat{n}(q)=\alpha_{q}^{\dagger} \alpha_{q}$ de los modos que diagonalizan el Hamiltoniano, dado que $[\hat{n}(q), H]=0$. Estudiaremos en detalle uno de tales modelos en el Capítulo 2. Para un modelo integrable, sea cuadrático o no, se espera que las cantidades conservadas adicionales obstaculicen la termalización y que el estado final sea no térmico ${ }^{2}$. Aún así podemos esperar que, aunque la dinámica esté altamente constreñida por las cantidades conservadas, exista alguna especie de «ergodicidad» dentro de ese espacio restringido y que, por lo tanto, sea posible describir al estado final con alguna especie de ensamble estadístico. En este plan, la mejor apuesta que podemos hacer acerca de cuál va a ser el estado final contando únicamente con la información de cuál es el estado inicial y cuáles son las cantidades conservadas $I_{q}$ que consideramos relevantes viene dada por la matriz

\footnotetext{
${ }^{2}$ Esto puede depender de las condiciones iniciales. Se ha visto que en ciertos sistemas integrables, si el estado inicial es lo suficientemente complicado o si su energía es lo suficientemente alta, el estado final es térmicol23].
} 
densidad

$$
\rho_{G g}=\frac{e^{-\sum_{q} \lambda(q) I(q)}}{Z_{G g}},
$$

en donde $G g$ significa ensamble de Gibbs generalizado (GGE por sus siglas en inglés), $Z_{G g}=$ $\operatorname{Tr}\left[e^{-\sum_{q} \lambda(q) I(q)}\right]$ y los multiplicadores de Lagrange están fijados por las condiciones iniciales,

$$
\operatorname{Tr}[\rho(0) I(q)]=\operatorname{Tr}\left[\rho_{G g} I(q)\right] .
$$

La matriz densidad Ec. (1.8) es la matriz densidad que maximiza la entropía de Von Neumann $S=\operatorname{Tr}[\rho \log \rho]$ sujeta a los constraints Ec. (1.9) y representa, como se mencionó anteriormente, la mejor apuesta que podemos hacer acerca de cuál va a ser el estado final del sistema contando con la información acerca del valor de las cantidades conservadas. En este caso no estamos haciendo una descripción de mecánica estadística del estado final del sistema a la manera de Gibbs con la maquinaria de los ensambles estadísticos y el teorema ergódico, sino que más bien estamos haciendo un ejercicio de inferencia lógica en condiciones de información incompleta. Este enfoque de la mecánica estadística (y de la física en general) fue defendido brillantemente por Jaynes [70,71]. Con este preámbulo debería quedar claro que $a$ priori la matriz densidad Ec. (3.80) no tiene por qué representar el estado final del sistema. Sin embargo, para modelos cuadráticos o que pueden mapearse a un modelo cuadrático (el modelo de Ising 1D en un campo transverso, cadena XY, hard core bosons, etc.) puede demostrarse en general [31, 3] que, en el límite termodinámico, si $A$ es un subsistema de nuestro sistema total, $\bar{A}$ es su complemento y $\rho(t)$ es la matriz densidad del sistema completo,

$$
\lim _{t \rightarrow \infty} \operatorname{Tr}_{\bar{A}}[\rho(t)]=\operatorname{Tr}_{\bar{A}}\left[\rho_{G g}\right],
$$

es decir, todas las matrices densidad reducidas quedan descriptas por el GGE. El mecanismo de relajación al GGE en este caso es el dephasing, el cual analizaremos en detalle en el capítulo siguiente. Resulta suficiente tomar como cantidades conservadas sólo a los números de ocupación $\hat{n}(q)$ de los modos que diagonalizan el Hamiltoniano, es decir, no es necesario considerar, por ejemplo, productos de esos números de ocupación, los cuales, sin embargo, también son cantidades conservadas. Además de la prueba formal de su validez, el GGE ha sido verificado explícitamente en una gran número de modelos cuadráticos [124, 17, 3, 18], incluyendo el modelo de Luttinger [30,67], el ejemplo particular que analizaremos en el capítulo siguiente. En el caso de modelos integrables que no pueden mapearse a un modelo cuadrático tales como aquellos que pueden resolverse a través del ansatz de Bethe (modelo XXZ, modelo de Lieb-Liniger, etc.) existe una cantidad importante de resultados que apuntan a la validez del GGE $[44,108,98]$. Sin embargo, la elección del conjunto mínimo de cantidades conservadas necesarias para construir el ensamble de Gibbs generalizado que describa el estado asintótico del sistema es aún un problema abierto [142] y, más importante, una prueba general de su validez aún no ha sido descubierta. En particular, no se han descubierto las condiciones necesarias o suficientes que deben cumplir tanto el estado inicial como el 
Hamiltoniano de evolución para asegurar la validez del GGE. Por ejemplo, en la Ref. [ 142] se estudió un quench en la cadena de Heisenberg anisotrópica con un estado de Neel inicial y se encontró que el GGE construído con las cantidades conservadas que provee el ansatz de Bethe no describe correctamente el estado asintótico de tiempos largos.

\subsection{Hipótesis de Autoestados Termalizados}

En sistemas clásicos el mecanismo fundamental para alcanzar el equilibrio térmico en sistemas aislados es la ergodicidad. En sistemas cuánticos, el mecanismo fundamental detrás de la termalización es uno de los problemas abiertos más importantes en esta área [111]. Una de las propuestas más populares es la Hipótesis de Autoestados Termalizados (ETH por sus siglas en inglés)de Deutsch y Srednicki [38, 134]. Para formular este escenario, consideremos que la expansión del estado inicial $\left|\psi_{0}\right\rangle$ en la base de autoestados $\left|\Psi_{\alpha}\right\rangle$ del Hamiltoniano de evolución $H$ es

$$
\left|\psi_{0}\right\rangle=\sum_{\alpha} C_{\alpha}\left|\Psi_{\alpha}\right\rangle
$$

en donde $C_{\alpha}$ son ciertos coeficientes complejos. La evolución de la función de onda del sistema es

$$
|\psi(t)\rangle=e^{-i H t}\left|\psi_{0}\right\rangle=\sum_{\alpha} C_{\alpha} e^{-i E_{\alpha} t}\left|\Psi_{\alpha}\right\rangle,
$$

en donde $E_{\alpha}$ son las autoenergías de $H$. La evolución temporal del valor de expectación de un dado operador $A$ vendrá dada por

$$
\langle A(t)\rangle \equiv\langle\psi(t)|A| \psi(t)\rangle=\sum_{\alpha, \beta} C_{\alpha}^{*} C_{\beta} e^{i\left(E_{\alpha}-E_{\beta}\right) t} A_{\alpha \beta}
$$

con $A_{\alpha \beta} \equiv\left\langle\Psi_{\alpha}|A| \Psi_{\beta}\right\rangle$. El promedio a tiempos largos del observable resulta entonces

$$
\lim _{T \rightarrow \infty} \frac{1}{T} \int_{0}^{T} d t\langle A(t)\rangle=\sum_{\alpha}\left|C_{\alpha}\right|^{2} A_{\alpha \alpha}
$$

En ausencia de degeneraciones en el espectro, si el valor del observable relaja a algún valor fijo, debe hacerlo a (1.14). La afirmación de que el ensamble microcanónico reproduce el promedio de tiempos largos del valor de expectación puede escribirse como

$$
\sum_{\alpha}\left|C_{\alpha}\right|^{2} A_{\alpha \alpha}=\langle A\rangle_{\text {microcan. }}\left(E_{0}\right) \equiv \frac{1}{\mathscr{N}_{E_{0}, \Delta E}} \sum_{\left|E_{0}-E_{\alpha}\right|<\Delta E} A_{\alpha, \alpha}
$$

en donde $E_{0}=\left\langle\psi_{0}|H| \psi_{0}\right\rangle$ es la energía dele estado inicial, $\Delta E$ es la ventana microcanónica de energía (suficientemente grande como para incluir una cantidad macroscópica de estados pero pequeña comparada con las escalas de energía del sistema) y la normalización $\mathscr{N}_{E_{0}, \Delta E}$ es el número de estados dentro de esa ventana de energía alrededor de $E_{0}$. En el límite 
termodinámico los niveles están infinitamente juntos y debemos reemplazar sumas por integrales convenientemente. La universalidad termodinámica es evidente en esta igualdad: mientras que el miembro izquierdo depende de las condiciones iniciales a través de los $C_{\alpha}$, el miembro derecho depende sólo de la energía del estado inicial. Uno de los mecanismos que puede explicar esta universalidad es la ETH:

Los valores de expectación en autoestados $A_{\alpha \alpha}$ prácticamente no fluctúan entre autoestados cercanos en energía.

Dado que $\frac{1}{\mathcal{N}_{E_{0}, \Delta E}} \sum_{\left|E_{0}-E_{\alpha}\right|<\Delta E} 1=\sum_{\alpha}\left|C_{\alpha}\right|^{2}=1$ el promedio de tiempos largos y el promedio microcanónico resultan iguales con la única condición de que la distribución de energías del estado inicial sea lo suficientemente angosta. Existen también otros mecanismos posibles para realizar esta igualdad, pero se ha comprobado que la ETH es el mecanismo detrás de la termalización en varios sistemas (ver [125] y sus referencias).

Se ha demostrado que la termalización y la ETH dejan de valer para sistemas estrictamente integrables (finitos o en el límite termodinámico) y cerca de un punto integrable en sistemas finitos [121]. En particular, se ha visto que cuanto más pequeño sea el sistema es necesaria una ruptura de integrabilidad más grande para que la ETH y con ella la termalización, funcionen. Se supone que en el límite termodinámico una ruptura infinitesimal de la integrabilidad basta para que la ETH valga y para que el sistema, por lo tanto, termalice. Recientemente se ha aportado fuerte evidencia a favor de esta conjetura [122]. Por otro lado, los indicadores de caos cuántico, como ciertas propiedades estadísticas del autoespectro del Hamiltoniano, también cambian abruptamente ante una ruptura infinitesimal de la integrabilidad en el límite termodinámico [129].

\subsection{Tipicalidad canónica}

Existe una visión sobre las bases de la mecánica estadística y la termalización cuántica que no invoca promedios sobre ensambles o promedios temporales. Es una visión puramente cuántica que no tiene análogo clásico y fue propuesta por Popescu y colaboradores en la Ref. [115]. Consideremos que el estado cuántico de un sistema aislado grande, que llamaremos el universo, es un estado puro. Es decir, no hay carencia de información subjetiva sobre el estado del universo, como la que estaría codificada en las probabilidades clásicas que son utilizadas para construir matrices densidades de estados mezcla. La entropía del sistema es cero. Sin embargo, al considerar una parte del universo, a la que llamaremos sistema $(S)$, es posible que su estado no sea puro debido al entrelazamiento cuántico con el resto del universo (el entorno $E$ ). Por lo tanto, hay una carencia objetiva de conocimiento acerca del estado del sistema, a pesar de que sepamos todo acerca del estado del universo. La entropía del sistema es diferente de cero. Lo que fue probado en [115] es que la termalización es una característica genérica de los estados puros del universo, en el sentido de que para casi cualquier estado puro del universo, el estado reducido del sistema es un estado canónico mezcla como los 
considerados al hablar sobre la definición de termalización.

Más concretamente, consideremos que el estado del universo obedece una ligadura global $R$ (que su energía tenga un valor definido, por ejemplo). Es posible representar tal ligadura restringiendo el estado del sistema $S$ y del entorno $E$ a un cierto subespacio $\mathscr{H}_{R}$ del espacio de Hilbert total $\mathscr{H}_{R} \subseteq \mathscr{H}_{E} \otimes \mathscr{H}_{S}$. Se define $\mathscr{E}_{R}$, el estado equiprobable del universo correspondiente a la restricción $R$, como

$$
\mathscr{E}_{R}=\frac{\mathbf{1}_{R}}{d_{R}}
$$

en donde $\mathbf{1}_{R}$ es el operador identidad en $\mathscr{H}_{R} \mathrm{y} d_{R}$ es su dimensión. $\mathscr{E}_{R}$ es el estado máximamente mezclado en $\mathscr{H}_{R}$, en el cual cada estado puro tienen la misma probabilidad. Por otro lado, se define el estado canónico del sistema $\Omega_{S}$ correspondiente a la ligadura $R$ al estado del sistema correspondiente al estado $\mathscr{E}_{R}$ del universo:

$$
\Omega_{S}=\operatorname{Tr}_{E}\left[\mathscr{E}_{R}\right]
$$

En la Ref. [115] se utilizó el lema de Levy para demostrar que para casi cualquier estado puro del universo, el estado reducido del sistema está muy cerca (según una distancia bien definida en el espacio de las matrices densidad) al estado canónico $\Omega_{S}$. Es decir, para casi cualquier estado puro del universo, el sistema se comporta como si el universo estuviera en el estado $\mathscr{E}_{R}$. La conexión con la mecánica estadística viene de considerar que la ligadura sobre el estado del universo es la energía. En tal caso puede mostrarse que el estado canónico del sistema resulta ser $\Omega_{S} \propto \operatorname{Tr}_{E}\left[e^{-\beta H_{U}}\right]$, en donde $H_{U}$ es el Hamiltoniano del universo y $\beta$ es la temperatura que queda fija al elegir un dado valor de la energía para el universo. Es decir, en esta perspectiva, la termalización de un sistema cuántico aislado no es demostrada en base a consideraciones dinámicas, es decir, infiriendo las características del estado final a partir de la condición inicial utilizando la dinámica del sistema (como en el enfoque de la ETH), sino que, en cambio, se utiliza el hecho de que, dado que casi para cualquier estado puro del universo sus subsistemas se encontrarán en un estado canónico, puede anticiparse que casi cualquier evolución que el universo realice a partir de una condición inicial atípica (aquella en que los subsistemas no se encuentran en el estado canónico) llevará a un estado en el que los sistemas están en un estado canónico.

Como hemos visto antes, los sistemas integrables en general no termalizan. Sería interesante entonces ver cómo se entiende esto desde el punto de vista de la tipicalidad canónica. Posiblemente el argumento falle debido que para un sistema integrable no es suficiente considerar una sola ligadura $R$ (como la energía) sino un número extensivo de ellas.

\subsection{Dinámica de no equilibrio}

Una vez que hemos visto que el estado asintótico de un sistema cuántico aislado puede parecerse a un estado térmico y cuáles son los mecanismos detrás de ese fenómeno, vale 
preguntarse cómo llega el sistema a tal estado térmico, es decir, cuál es la dinámica de tiempos finitos. Este es el tema fundamental que será desarrollado a lo largo de la tesis. A grandes rasgos, existen tres escenarios posibles para la dinámica:

1. No hay relajación, $\rho_{\infty}$ no existe.

2. Relajación en una sola etapa, no hay estados estacionarios intermedios.

3. Relajación en dos o más etapas, hay estados estacionarios intermedios entre la condición inicial y el estado térmico final.

En esta tesis estaremos interesados en particular en los escenarios 2 y 3 . En el Capítulo 3 mostraremos cómo emergen estados metaestables de tiempos cortos llamados estados pretermalizados (escenario 3). En el Capítulo 5 analizaremos la vida media de esos estados metaestables, incluyendo el estudio de las regiones de parámetros en las que están ausentes (escenario 2). En particular, si bien en el límite termodinámico sólo una rotura infinitesimal de la integrabilidad es necesaria para asegurar que las propiedades de tiempos largos sean térmicas, veremos que la dinámica del sistema hacia el estado térmico es cualitativamente diferente lejos de la integrabilidad y cerca de ella.

\subsection{Algunos experimentos notables}

Como fue mencionado en la Introducción, el estudio de sistemas cuánticos aislados fuera de equilibrio ha sido impulsado, en gran medida, por los avances en los experimentos con átomos fríos cargados en redes ópticas. Haremos aquí una breve reseña de los experimentos más importantes.

Quizás el primer trabajo experimental que explotó la capacidad de los átomos fríos de simular la dinámica de no equilibrio de sistemas cuánticos aislados fue el trabajo de Greiner et al. [55] en el que se observó el colapso y resurgimiento de la amplitud de la función de onda colectiva de un condensado de Bose-Einstein luego de cambiar abruptamente la intensidad de la interacción entre los átomos del condensado.

Sin embargo, el experimento que gatilló la mayor cantidad de actividad teórica en este campo fue el de Kinoshita et al. [76]. En ese experimento un arreglo de tubos unidimensionales de átomos de ${ }^{87} R b$ fue preparado en una superposición de estados con momento opuesto. Luego se dejaba evolucionar a cada uno de las réplicas del sistema y se monitereaba la distribución de momentos, promediada sobre todas las réplicas del sistema, a diferentes tiempos de evolución. Incluso después de escalas de tiempo suficientemente grandes como para que se produjeran miles de colisiones entre los átomos, la distribución de momentos no relajó a la forma correspondiente al equilibrio térmico (ver Fig. 1.1). Probablemente, la explicación teórica de los resultados de este experimento se encuentra en el hecho de que el sistema experimental utilizado era una realización prácticamente perfecta del modelo de Lieb-Liniger, 
un modelo unidimensional de bosones interactuantes via un potencial de contacto. Este modelo es uno de los ejemplos más conocidos de sistema integrable que no puede mapearse a un modelo cuadrático. La falta de termalización fue atribuída entonces a la presencia de un gran número de cantidades conservadas durante la evolución.

Otro experimento representativo en este campo es el de Cheneau et al. [33] en el que se estudió la propagación de correlaciones luego de cambiar abruptamente la intensidad de las interacciones en un gas de átomos de Rubidio. En este experimento se pudo observar experimentalmente el llamado efecto cono de luz que se genera debido a que las correlaciones luego de un quench se propagan a una velocidad finita. Analizaremos este importante efecto en el capítulo siguiente.

Finalmente, mencionaremos el estudio de la Ref. [139], en el que se observó la relajación de un gas de bosones ultrafríos a partir de un estado inicial inhomogéneo. En este trabajo se pudo observar la relajación al estado térmico en escalas de tiempo menores que la duración del experimento para interacciones lo suficientemente débiles. Las curvas experimentales obtenidas en este trabajo se comparan muy bien con los resultados de la técnica del grupo de renormalización de la matriz densidad dependiente del tiempo (t-DMRG). Lo que es más interesante es que los resultados experimentales permiten acceder a tiempos más largos que las simulaciones numéricas, dando el primer paso en el programa de la "simulación cuántica», es decir, la utilización de sistemas de átomos fríos para investigar las propiedades de sistemas de muchos cuerpos de manera más eficiente que lo que es actualmente posible usando sistemas de cómputo clásicos (ver Fig. 1.2). En el Capítulo 5 analizaremos teóricamente una situación parecida a la configuración de este experimento. 


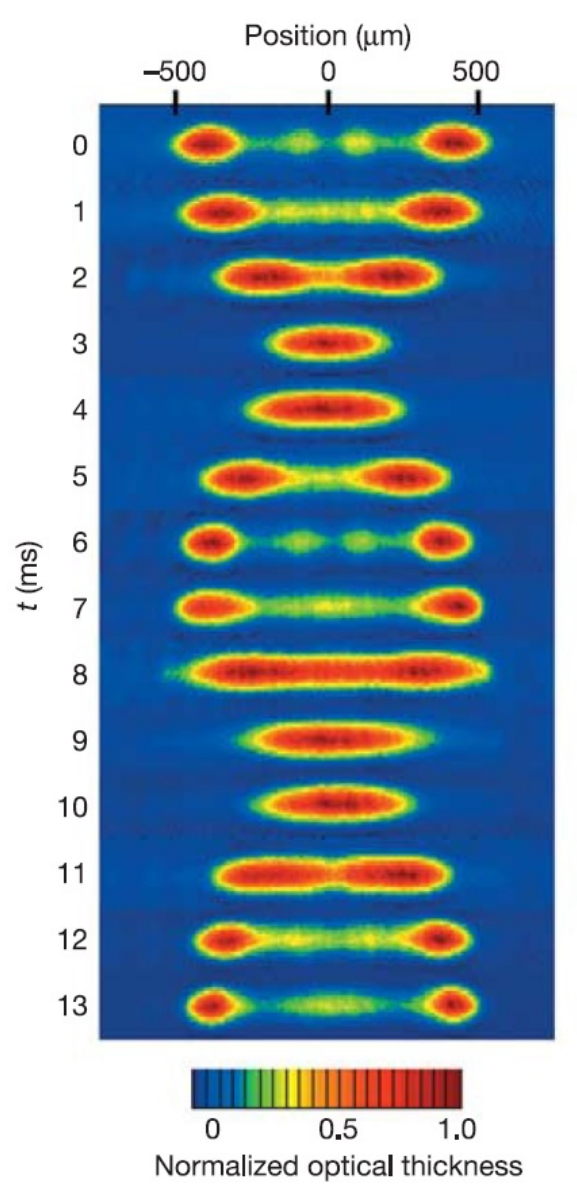

Figura 1.1: Experimento de Kinoshita et al. Diferentes estadíos de la evolución de un gas de bosones fuertemente interactuantes preparados inicialmente en dos nubes con momentos opuestos. Las imágenes son gráficos de color falso de la densidad de átomos en el sistema unidimensional. El sistema se encuentra dentro de una trampa parabólica, ló que implica que el estado de equilibrio correspondería a tener una sola nube de átomos en el centro de la trampa. Se observa que aún luego de varias oscilaciones este estado no es alcanzado. Tomado de [76]. 


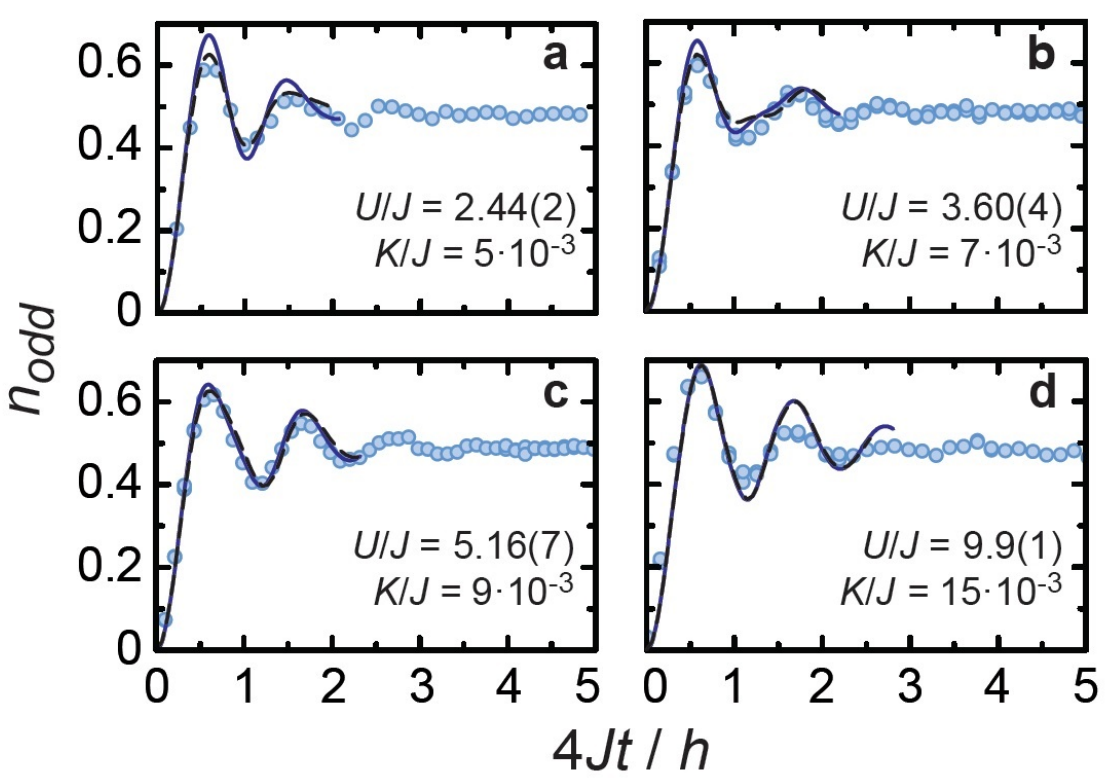

Figura 1.2: En el experimento de la Ref. [139] se prepara inicialmente un estado inhomogéneo de átomos de ${ }^{87} R b$ (bosones) en una red óptica unidimensional, tal que los sitios pares contienen un átomo y los impares están vacíos. Luego se deja evolucionar al sistema con un Hamiltoniano invariante traslacional con interacciones de corto alcance (que puede modelarse con el Hamiltoniano de Hubbard). En la figura se muestra la relajación de la densidad de átomos en un sitio impar para diferentes intensidades de la interacción $U / J$, mostrando que el sistema tiende rápidamente al estado térmico con un promedio de $1 / 2$ bosón por sitio. Los círculos celestes son los datos experimentales mientras que las líneas puntedas y contínuas son simulaciones de DMRG. Puede verse claramente el acuerdo entre los resultados experimentales y teóricos y, lo que es más, que los datos experimentales alcanzan escalas de tiempo más allá de lo que puede predecirse confiablemente usando las simulaciones numéricas. Tomado de [139]. 


\section{Dephasing y el ensamble de Gibbs generalizado}

Un rasgo universal de la dinámica fuera de equilibrio de sistemas cuánticos aislados es la dinámica de desfasaje o dephasing a tiempos cortos. Este régimen es análogo a la expansión balística que ocurre en los primeros instantes de tiempo de la evolución de líquidos, vidrios y otros fluidos clásicos. El dephasing es un efecto típico que surge de sumar un cierto conjunto de funciones oscilantes del tiempo con un espectro de frecuencias lo suficientemente denso: en los instantes iniciales todas las oscilaciones están aproximadamente en fase, pero a medida que pasa el tiempo comienzan a «desfasarse» debido a la diferencia de frecuencias y su promedio decae a cero. En el caso de quenches débiles el comportamiento oscilante viene dado por la dinámica de ciertos modos cuasilibres de baja energía que son excitados con el quench. Sin embargo, el concepto no está restringido a quenches débiles. Por ejemplo, el dephasing puede surgir al analizar la dinámica de modelos de campo medio [57] que, si bien tienen Hamiltonianos cuadráticos, pueden modelar sistemas con interacciones fuertes, lo que permite estudiar quenches fuertes [130].

El dephasing es un mecanismo que permite al sistema perder rápidamente memoria de las condiciones iniciales, al menos al nivel de ciertas funciones de correlación simples, es decir, genera una dinámica efectivamente irreversible en el sistema aislado.Sin embargo, en general, el dephasing conduce a estados estacionarios que no son térmicos sino que todavía guardan mucha memoria del estado inicial. De hecho, si a tiempos cortos podemos describir un sistema como un conjunto de excitaciones cuasilibres (aproximadamente no interactuantes en una cierta escala de tiempo de la dinámica) de baja energía, eso quiere decir que existen, al menos en la descripción efectiva, un número extensivo de cantidades conservadas, los números de ocupación de tales modos. La descripción efectiva del sistema es, por lo tanto, integrable. Al intentar una descripción estadística de los estados estacionarios que son alcanzados como fruto del dephasing debemos tener en cuenta la presencia de todas esas cantidades conservadas. Los ensambles construidos de esa manera son llamados ensambles de Gibbs generalizados. Por su misma naturaleza, la dinámica de dephasing, que domina a tiempos cortos por sobre otros mecanismos de relajación tales como las colisiones inelásticas, es independiente de los detalles de la interacción. 
En este capítulo ejemplificaremos y profundizaremos sobre estos conceptos usando un modelo paradigmático de la física cuántica en una dimensión (1D), el modelo de Luttinger (ML). Este modelo de fermiones interactuantes no presenta excitaciones partícula-agujero renormalizadas como sus contrapartes en dimensión mayor, sino que las excitaciones del sistema son únicamente colectivas (holones y spinones) y pueden representarse por medio de un conjunto de modos bosónicos no interactuantes. Dado que el el modelo bosónico es cuadrático, el dephasing entre estos modos constituye el único mecanismo de relajación del sistema. El ML no es sólo un modelo de juguete en el que pueden calcularse todas las funciones de correlación, sino que, en equilibrio este modelo es el punto fijo del grupo de renormalización de una gran variedad de modelos unidimensinonales que no poseen gaps en sus espectros. Fuera de equilibrio, como veremos más adelante, la dinámica de dephasing de los modos bosónicos del NL también captura la dinámica a tiempos cortos de estos modelos.

En este capítulo, todos los resultados relacionados con el quench de interacción en el ML con interacciones de Coulomb [101] y la comparación con un modelo en la red [102] constituyen aportes originales de esta tesis. Más allá de los resultados particulares, el panorama conceptual presentado también es novedoso.

\subsection{El modelo de Luttinger y bosonización}

El ML describe un sistema de fermiones interactuantes en una dimensión [137, 88, 92, 51]. La simplificación clave del modelo consiste en que suypone una relación de dispersión (desnuda) lineal, la cual induce una clara separación enre partículas que se mueven hacia la derecha y hacia la izquierda. El Hamiltoniano del ML es

$$
H_{L M}=H_{0}+H_{2}+H_{4}
$$

en donde

$$
H_{0}=\sum_{q, r=R, L} v_{F} q: c_{q, r}^{\dagger} c_{q, r}:
$$

es la parte libre de $H_{L M}$. Aquí, $c_{q, r}^{\dagger}$ y $c_{q, r}$ son operadores que crean o destruyen fermiones con momento $q . v_{F}$ es la velocidad de Fermi. En principio, asumimos un sistema finito de longitud $L$ con condiciones de contorno periódicas, pero estaremos interesados mayormente en el límite termodinámico $L \rightarrow \infty$. El índice $r$ etiqueta quiralidad de las partículas, y sus posibles valores son $L$ (rama izquierda del espectro) y $R$ (rama derecha). El orden normal fermiónico denotado con :.... : es necesario para remover de los valores de expectación las conrtibuciones infinitas debidas al hecho de que el estado fundamental del modelo es un mar de Fermi [59], es decir, el estado en el cual todos los niveles de una partícula con $q<0$ están ocupados.

El scattering entre partículas es parametrizado usando dos funciones, $g_{2}(q)$ and $g_{4}(q)$, que 
están relacionadas con los procesos que intercambian fermiones entre las dos ramas del espectro y aquellos que dejan a los fermiones en su rama, respectivamente. En términos de estas funciones, la parte de interacción del $H_{L M}$ es

$$
\begin{aligned}
& H_{2}=\frac{1}{L} \sum_{q} g_{2}(q): \rho_{q R} \rho_{q L}: \\
& H_{4}=\frac{1}{2 L} \sum_{q} g_{4}(q): \rho_{q R} \rho_{-q R}+\rho_{q L} \rho_{-q L}:
\end{aligned}
$$

en donde hemos definido densidades en el espacio de momentos como $\rho_{q r}=\sum_{k}: c_{k-q, r}^{\dagger} c_{k r}$ : Siempre consideraremos el caso $g_{2}(q)=g_{4}(q)=V(q)$, en donde $V(q)$ es la transformada de Fourier del potencial de interacción entre los fermiones $V(x)$. Para más detalles acera de este modelo pueden consultarse los primeros capítulos de la Ref. [51].

Las excitaciones del sistema son quasipartículas bosónicas [92, 51]. Para constatar esto, primero notemos que los operadores densidad $\rho_{q r}$ obedecen las siguientes reglas de conmutación,

$$
\left[\rho_{q r}^{\dagger}, \rho_{r^{\prime} q^{\prime}}\right]=-r \delta_{r r^{\prime}} \delta_{q q^{\prime}} n_{q}
$$

en donde hemos definido el entero $n_{q}=\frac{L q}{2 \pi}$. El álgebra especial de la Eq. (2.5) puede transformarse en el álgebra bosónica usual introduciendo los siguientes operadores:

$$
b(q)=\frac{1}{\sqrt{\left|n_{q}\right|}} \begin{cases}\rho_{q R}, & \text { for } q>0 \\ \rho_{q L}, & \text { for } q<0 .\end{cases}
$$

Nótese que las componentes con $q=0$ (modos cero) requieren un tratamiento separado dado que $\rho_{0 r}=N_{r}$ es la desviación, en relación con el estado fundamental, del número de fermiones de una dada quiralidad. Es usual introducir las combinaciones

$$
N=N_{R}+N_{L}, \quad J=N_{R}-N_{L}
$$

En términos de estos operadores y de los operadores bosónicos introducidos en Eq. (2.6) el 
Hamiltoniano del ML se escribe

$$
\begin{aligned}
H_{0}= & \sum_{q \neq 0} v_{F}|q| b_{q}^{\dagger} b_{q}+\frac{\pi v_{F}}{2 L}\left(N^{2}+J^{2}\right), \\
H_{2}= & \frac{1}{2} \sum_{q \neq 0} V(q)|q|\left[b_{q} b_{-q}+b_{q}^{\dagger} b_{-q}^{\dagger}\right] \\
& +\frac{\pi V(0)}{2 L}\left(N^{2}-J^{2}\right) \\
H_{4}= & \sum_{q \neq 0} V(q)|q| b_{q}^{\dagger} b_{q} \\
& +\frac{\pi V(0)}{2 L}\left(N^{2}+J^{2}\right) .
\end{aligned}
$$

Ignorando la parte de los modos cero (que no va a contribuir en el límite termodinámico $L \rightarrow \infty$ ), podemos diagonalizar el Hamiltoniano bosónico utilizando una transformación de Bogoliubov, introduciendo nuevos operadores $a_{q}$ y $a_{q}^{\dagger}$ de la siguiente manera:

$$
\left(\begin{array}{c}
a_{q} \\
a_{-q}^{\dagger}
\end{array}\right)=\left(\begin{array}{cc}
\cosh \theta(q) & \sinh \theta(q) \\
\sinh \theta(q) & \cosh \theta(q)
\end{array}\right)\left(\begin{array}{c}
b_{q} \\
b_{-q}^{\dagger}
\end{array}\right),
$$

en donde el parámetro de la transformación $\theta(q)$ satisface la relación

$$
\tanh 2 \theta(q)=\frac{V(q)}{2 \pi v_{F}+V(q)} .
$$

En términos de los nuevos operadores el Hamiltoniano toma forma diagonal:

$$
H_{L M}=H_{0}+H_{2}+H_{4}=\sum_{q \neq 0} \epsilon(q) a_{q}^{\dagger} a_{q}+\text { modos cero, }
$$

en donde $\epsilon(q)=v_{F}|q| \sqrt{1+2 \frac{V(q)}{\pi \nu_{F}}}$ es la relación de dispersión de los modos bosónicos. Vemos entonces que el ML es equivalente a un conjunto de osciladores armónicos. Es posible encontrar una expresión para el campo fermiónico completamente en función de los campos bosónicos. Definiendo el campo fermiónico $\psi_{r}(x)=\frac{1}{s q r t L} \sum_{q} e^{i q x} c_{q, r}$ es posible mostrar que

$$
\psi_{r}(x)=F_{r} \frac{1}{\sqrt{2 \pi a}} e^{-i \phi_{r}(x)}
$$

en donde

$$
\phi_{r}(x)=-r \sum_{q \neq 0} \frac{e^{-a q / 2}}{\sqrt{n_{q}}}\left(e^{i r q x} b_{q r}+e^{-i r q x} b_{q r}^{\dagger}\right)
$$

con $a \rightarrow 0^{+1}$ y los $F_{r}$, llamados generalmente factores de Klein, son operadores unitarios

\footnotetext{
${ }^{1}$ Para implementar la bosonización es útil introducir a mano un cutoff ultravioleta $a$ [51]. Dado que para
} 
que satisfacen $\left\{F_{r}^{\dagger}, F_{r^{\prime}}\right\}=2 \delta_{r r^{\prime}}$. La llamada identidad de bosonización, Eq. (2.16), junto con el carácter cuadrático del Hamiltoniano bosónico permiten calcular todas las funciones de correlación del modelo en equilibrio. Esto es de una importancia particular dado que el ML es el punto fijo del grupo de renormalización de un gran conjunto de modelos sin gap en una dimensión [59, 58], llamados Líquidos de Luttinger.

\subsection{Quenches en la interacción y dephasing}

Para analizar la dinámica fuera de equilibrio del ML utilizaremos un protocolo bastante particular, un quench en el alcance de la interacción. Consideremos que a $t=0$ el sistema está preparado en el estado fundamental $|G S\rangle_{i}$ del Hamiltoniano $H_{i}$, definido según la Ec. (2.1) con un potencial de interacción $V_{i}(q)$. El Hamiltoniano que en adelante genera la dinámica del sistema, $H_{f}$, tiene un potencial de interacción diferente $V_{f}(q)$. Al nivel de la representación bosónica, ambos Hamiltonianos pueden ponerse en forma diagonal por medio de sendas transformaciones de Bogoliubov caracterizadas por parámetros $\theta_{i}(q)$ y $\theta_{f}(q)$, relacionadas con los respectivos potenciales por la Ec. (2.14). La solución de la dinámica se reduce entonces a un cambio de base realizado por una serie de transformaciones de Bogoliubov anidadas, de manera de encontrar la dependencia temporal de los operadores que diagonalizan la versión bosónica de $H_{f}$.

Definiremos $\left\{a_{q}, a_{-q}^{\dagger}\right\}\left(\left\{\alpha_{q}, \alpha_{-q}^{\dagger}\right\}\right)$ como los operadores que diagonalizan $H_{i}\left(H_{f}\right)$. Dado que trabajaremos en la representación de Heisenberg, la evolución del sistema puede calcularse conociendo la dependencia temporal de los operadores $\left\{a_{q}, a_{-q}^{\dagger}\right\}$, cuya acción sobre el estado inicial es conocida:

$$
a_{q}|G S\rangle_{i}=0
$$

Por otro lado los operadores $\left\{\alpha_{q}, \alpha_{-q}^{\dagger}\right\}$ son los modos libres de nuestro problema. Estos operadores poseen una dependencia temporal puramente oscilatoria $e^{i H_{f} t} \alpha_{q} e^{-i H_{f} t}=\alpha_{q}(t)=$ $\alpha_{q} \exp \left[-i \epsilon_{f}(q) t\right]$. Los operadores número de coupación asociados con tales operadores $n_{q} \equiv \alpha_{q}^{\dagger} \alpha_{q}$ son cantidades conservadas por la dinámica $\left[n_{q}, H_{f}\right]=0$. Por lo tanto, en la dinámica del ML hay $L$ cantidades conservadas.

Usaremos la forma explícita de las transformaciones de Bogoliubov que relacionan estos operadores con la base libre $\left\{b_{q}, b_{-q}^{\dagger}\right\}$ definida más arriba:

$$
\left(\begin{array}{c}
a_{q} \\
a_{-q}^{\dagger}
\end{array}\right)=\mathscr{M}^{(i)}\left(\begin{array}{c}
b_{q} \\
b_{-q}^{\dagger}
\end{array}\right),
$$

potenciales bien comportados las integrales resultantes están bien definidas es posible tomar el límite $a \rightarrow 0$ al final de los cálculos sin mayores problemas. 
y

$$
\left(\begin{array}{c}
\alpha_{q} \\
\alpha_{-q}^{\dagger}
\end{array}\right)=\mathscr{M}^{(f)}\left(\begin{array}{c}
b_{q} \\
b_{-q}^{\dagger}
\end{array}\right),
$$

en donde

$$
\mathscr{M}^{(l)}=\left(\begin{array}{cc}
\cosh \left(\theta_{l}(q)\right) & \sinh \left(\theta_{l}(q)\right) \\
\sinh \left(\theta_{l}(q)\right) & \cosh \left(\theta_{l}(q)\right)
\end{array}\right)
$$

Con $l=i, f$. La transformación que relaciona la base inicial y la final es entonces:

$$
\begin{aligned}
& \left(\begin{array}{c}
a_{q} \\
a_{-q}^{\dagger}
\end{array}\right)=\mathscr{M}^{(i)}\left(\mathscr{M}^{(f)}\right)^{-1}\left(\begin{array}{c}
\alpha_{q} \\
\alpha_{-q}^{\dagger}
\end{array}\right) \\
& =\left(\begin{array}{cc}
\cosh \left(\theta_{f}(q)-\theta_{i}(q)\right) & \sinh \left(\theta_{f}(q)-\theta_{i}(q)\right) \\
\sinh \left(\theta_{f}(q)-\theta_{i}(q)\right) & \cosh \left(\theta_{f}(q)-\theta_{i}(q)\right)
\end{array}\right)\left(\begin{array}{c}
\alpha_{q} \\
\alpha_{-q}^{\dagger}
\end{array}\right) .
\end{aligned}
$$

Utilizando la dependencia temporal de los operadores $\left\{\alpha_{q}, \alpha_{-q}^{\dagger}\right\}$, llegamos a la solución formal de la dinámica:

$$
a_{q}(t)=f(q, t) a_{q}+g^{*}(q, t) a_{-q}^{\dagger}
$$

en donde

$$
\begin{aligned}
& f(q, t)=\cos \left(\epsilon_{f}(q) q t\right)-i \sin \left(\epsilon_{f}(q) t\right) \cosh \left[2\left(\theta_{f}(q)-\theta_{i}(q)\right)\right], \\
& g(q, t)=i \sin \left(\epsilon_{f}(q) q t\right) \sinh \left[2\left(\theta_{f}(q)-\theta_{i}(q)\right)\right] .
\end{aligned}
$$

Ahora utilicemos la solución para calcular la evolución de algunas cantidades relevantes. Comencemos con la densidad de energía cinética $e_{k i n}(t)=\frac{1}{L}{ }_{i}\left\langle G S\left|e^{i H_{f} t} H_{0} e^{-i H_{f} t}\right| G S\right\rangle_{i}$. Por simplicidad, consideremos un estado inicial no interactuante, $\theta_{i}=0$. En tal caso la evolución de la energía cinética toma la forma simple:

$$
e_{k i n}(t)=\frac{\nu_{F}}{L} \sum_{q>0} q \sinh ^{2}\left(2 \theta_{f}(q)\right)\left(1-\cos \left(2 \epsilon_{f}(q) t\right)\right) .
$$

Vemos entonces que la dinámica de este observable consiste en la superposición de términos oscilantes, cada uno de los cuales está relacionado con uno de los modos libres de nuestro sistema. Pasando al límite termodinámico, en el cual las sumas sobre momento se transforman 
en integrales, obtenemos

$$
e_{k i n}(t)=\frac{v_{F}}{2 \pi} \int_{0}^{\infty} d q q \sinh ^{2}\left(2 \theta_{f}(q)\right)\left(1-\cos \left(2 \epsilon_{f}(q) t\right)\right)
$$

Para cualquier potencial bien comportado cerca de $q=0$ el término oscilante desaparece para tiempos grandes ya que estamos integrando una función bien comportada contra una función que oscila alrededor de cero con una frecuencia que es proporcional a $t$. Esto es el dephasing en acción. Por más que la dinámica sea Hamiltoniana y que, adicionalmente, contemos con un número extensivo de cantidades conservadas el observable $e_{k i n}(t)$ va irreversiblemente a un límite bien definido para $t \rightarrow \infty$. Debemos mencionar que existe un "límite bien definido" a $t \rightarrow \infty$ sólo si el sistem está en el límite termodinámico, un sistema a tamaño finito exhibirá recurrencias para tiempos $t \sim n / L$ donde $n$ es un entero.

Para un potencial de corto alcance dado por

$$
\sinh 2 \theta_{l}(q) \approx e^{-i R_{0} q}\left(K_{l}-K_{l}^{-1}\right) / 2, \quad \epsilon(q) \approx v_{l}(0) q
$$

en donde $l=i, f$ dado que la misma aproximación puede usarse para definir el Hamiltoniano inicial o el final, $R_{0}$ es el alcance del potencial, $v(q)$ es la velocidad renormalizada de los modos libres (cuya dependencia en el momento es despreciable) y $K_{l}$ es el llamado parámetro de Luttinger que parametriza la intensidad de la interacción, obtenemos

$$
e_{k i n}(t)-e_{k i n}(\infty) \simeq-\frac{v_{F}}{4 \pi} \frac{\left(K_{f}-K_{f}^{-1}\right)^{2}}{4} \frac{1}{4 v^{2}(0) t^{2}},
$$

para $t \gg v(0) / R_{0}$. Más adelante compararemos este resultado con la dinámica de un modelo en la red con interacciones de corto alcance.

Pasemos ahora a un ejemplo un poco más demandante desde el punto de vista técnico, el cálculo de la matriz densidad de una partícula (función de Green de tiempos iguales). Veremos que en este ejemplo, también, la relajación es provista por el dephasing. Consideraremos el caso en que la condición inicial viene dada por un gas de fermiones interactuando con un potencial de corto alcance descripto por la aproximación ad hoc Ec. (2.28), un estado inicial no interactuante puede recobrarse tomando $K_{i}=1$. La evolución subsecuente está dictada por un Hamiltoniano con interacción $V_{f}(q)$ y ángulo de Bogoliubov $\theta_{f}(q)$. La matriz densidad de una partícula se define como

$$
C_{\psi_{r}}(x, t)=\left\langle e^{i H_{f} t} \psi_{r}^{\dagger}(x) \psi_{r}(0) e^{-i H_{f} t}\right\rangle,
$$

La transformada de Fourier de esta función de correlación es la distribución de momentos instantánea de los fermiones, $n(k, t)$. Usando la identidad de bosonización Ec. 2.16, la fórmula de Baker-Hausdorff y el hecho de que, dado que estamos tratando con una teoría de bosones libres (Hamiltoniano cuadrático), $\left\langle e^{D}\right\rangle=e^{\langle D\rangle+\frac{1}{2}\left\langle(D-\langle D\rangle)^{2}\right\rangle}$ para cualquier operador $D$ lineal en los campos $b_{q}$, obtenemos el siguiente resultado para la matriz densidad en el límite 
termodinámico:

$$
C_{\psi_{r}}(x, t)=C_{\psi_{r}}^{(i)}(x) \exp \left\{\frac{\left(K_{i}+K_{i}^{-1}\right)}{2} \Phi_{1}(x, t)+\frac{\left(K_{i}-K_{i}^{-1}\right)}{2} \Phi_{2}(x, t)\right\},
$$

en donde las funciones

$$
\Phi_{1}(x, t)=-2 \int_{0}^{\infty} \frac{d q}{q} \sinh ^{2}\left(2 \theta_{f}(q)\right) \sin ^{2}\left(\epsilon_{f}(q) t\right)(1-\cos (q x))
$$

$\mathrm{y}$

$$
\Phi_{2}(x, t)=2 \int_{0}^{\infty} \frac{d q}{q} \cosh \left(2 \theta_{f}(q)\right) \sinh \left(2 \theta_{f}(q)\right) \sin ^{2}\left(\epsilon_{f}(q) t\right)(1-\cos (q x))
$$

son independientes de las condiciones iniciales. En estas ecuaciones

$$
C_{\psi_{r}}^{(i)}(x)=\frac{i}{2 \pi}|x|^{-1 / 2\left(K_{i}+K_{i}^{-1}\right)}
$$

es la función de correlación en $t=0$, es decir, la función de correlación en el estado fundamental de $H_{i}$. Examinando las expresiones para las funciones $\Phi(x, t)$ queda claro que la relajación temporal tiene la misma naturaleza que la encontrada anteriormente para la energía cinética, el dephasing entre los modos bosónicos libres. Al tomar el límite $t \rightarrow \infty$, el $\sin ^{2}$ se promedia a 1/2 y de esta manera emerge el estado estacionario final independiente del tiempo. También puede observarse que la dependencia temporal está "entrelazada" con la dependencia espacial, algo que dará lugar al llamado efecto horizonte, el cual será analizado más adelante.

Para futura referencia, consideremos un estado inicial no interactuante, para el cual las Ecs. (2.31)-(2.32) valen tomando $K_{i}=1$. En tal caso la distribución de momentos en el estado inicial es una función escalón con una discontinuidad en el momento de Fermi $k_{F}$ de magnitud $Z=1$. Dado que las interacciones tienden a disminuir tal discontinuidad $(Z=0$ para un líquido de Luttinger en equilibrio y $Z<1$ para un líquido de Fermi en equilibrio) resulta natural investigar la evolución temporal del salto $Z(t)$ de la distribución de momentos instantánea de los fermiones $n(k, t)$ en el momento de Fermi $k_{F}$. La definición de $Z(t)$ es

$$
Z(t)=\lim _{k \rightarrow k_{F}^{+}} n(k, t)-\lim _{k \rightarrow k_{F}^{-}} n(k, t),
$$

en donde $n(k, t)$ es la transformada de Fourier instantánea de la matriz densidad de una partícula Ec. (2.30). Para un Hamiltoniano final con interacciones de corto alcance parametrizadas por $R_{0}, K_{f}$ y $v_{f}$, para tiempos suficientemente cortos $R_{0} \ll 2 v_{f} t \ll x$ la matriz densidad de una partícula adquiere la forma $C_{\psi_{r}}(x, t)=\frac{i Z(t)}{2 \pi x}$, con $[67,120]$

$$
Z(t)=t^{-\frac{1}{4}\left(K_{f}^{2}-K_{f}^{-2}-2\right)}
$$

Vemos entonces que a tiempos cortos el sistema se comporta como un líquido de Fermi con un residuo de cuasipartícula que decae como una ley de potencias. Estos resultados, para el 
caso de interaccione de corto alcance fueron encontrados por Cazalilla e Iucci[30, 67]. En esta tesis nos concentraremos en otro potencial de interés: el potencial de Coulomb [101]. En ese caso asumimos $V_{f}(x)=\frac{e^{2}}{\sqrt{x^{2}+d^{2}}}$, cuya transformada de Fourier es $V_{f}(q)=2 e^{2} K_{0}(q d)$, en donde $K_{0}(\zeta)$ es la función de Bessel modificada de segunda especie de orden cero y $d$ es un cutoff de distancias cortas. Esta forma fenomenológica fue propuesta para describir la interacción de Coulomb no apantallada en cables semiconductores [53] aislados, en cuyo caso $d$ está asociado con las dimensiones transversales del cable. La descripción de las propiedades de equilibrio del Hamiltoniano con tales interacciones fue realizado por primera vez por Schulz [131] y continuado por otros [69, 141, 52]. El espectro bosónico es de tipo plasmónico,

$$
\epsilon_{f}(q)=|q| \nu_{F} \sqrt{1+2 g K_{0}(q d)} \sim|q| \log ^{1 / 2}\left(\frac{1}{q d}\right),
$$

$\operatorname{con} g=\frac{e^{2}}{\pi v_{F}}$. La forma logarítmica es un resultado aproximado para $q$ pequeño. La energía de los bosones va a 0 para $q \rightarrow 0$, mientras que su velocidad de grupo $v_{f}(q)=\frac{d \varepsilon_{f}(q)}{d q}$ diverge como $v_{F} \sqrt{1-2 g \log (q d)}$ para $q \ll d^{-1}$. En el caso del potencial de Coulomb obtenemos

$$
Z(t) \sim e^{-\frac{g}{4} \log ^{2}\left(2 v_{F} t / d\right)}
$$

que decae más rápido que una ley de potencias pero más lento que una exponencial. Por otro lado a orden dominante para el potencial de Coulomb también vale que $e_{k i n}(t)-e_{k i n}(\infty) \sim t^{-3}$, lo que demuestra que, en comparación con un potencial de corto alcance, el potencial de largo alcance modifica drásticamente algunos aspectos de la dinámica pero no todos.

\subsection{Ensamble generalizado de Gibbs}

Como ha sido mencionado en el primer capítulo algunas de las cuestiones fundamentales en el campo de la dinámica de no equilibrio de sistemas cuánticos aislados están relacionadas con la naturaleza del estado que alcanza el sistema luego de una evolución infinita. En esta sección atacaremos estas cuestiones y trataremos de darles una respuesta en el marco del ejemplo particular que estamos estudiando.

Para comenzar utilizaremos el ejemplo de un quench en el alcance de la interacción: en el estado inicial los fermiones interactúan con un potencial de corto alcance parametrizado por $R_{0}, K_{i} \mathrm{y} v_{i}$, mientras que el Hamiltoniano de evolución tiene interacciones de Coulomb, parametrizadas por $g$ y $d$. Es decir, el estado inicial es un líquido de Luttinger pero a $t=0$ se encienden interacciones de largo alcance entre los fermiones. Para investigar las propiedades del estado estacionario luego del quench, notamos que para $t \rightarrow \infty$ el factor oscilatorio dependiente del tiempo en las integrales Ecs. (2.32) and (2.33) se promedia a 1/2. Utilizando análisis asintótico podemos encontrar el comportamiento de distancias grandes $x \gg d$ de la 


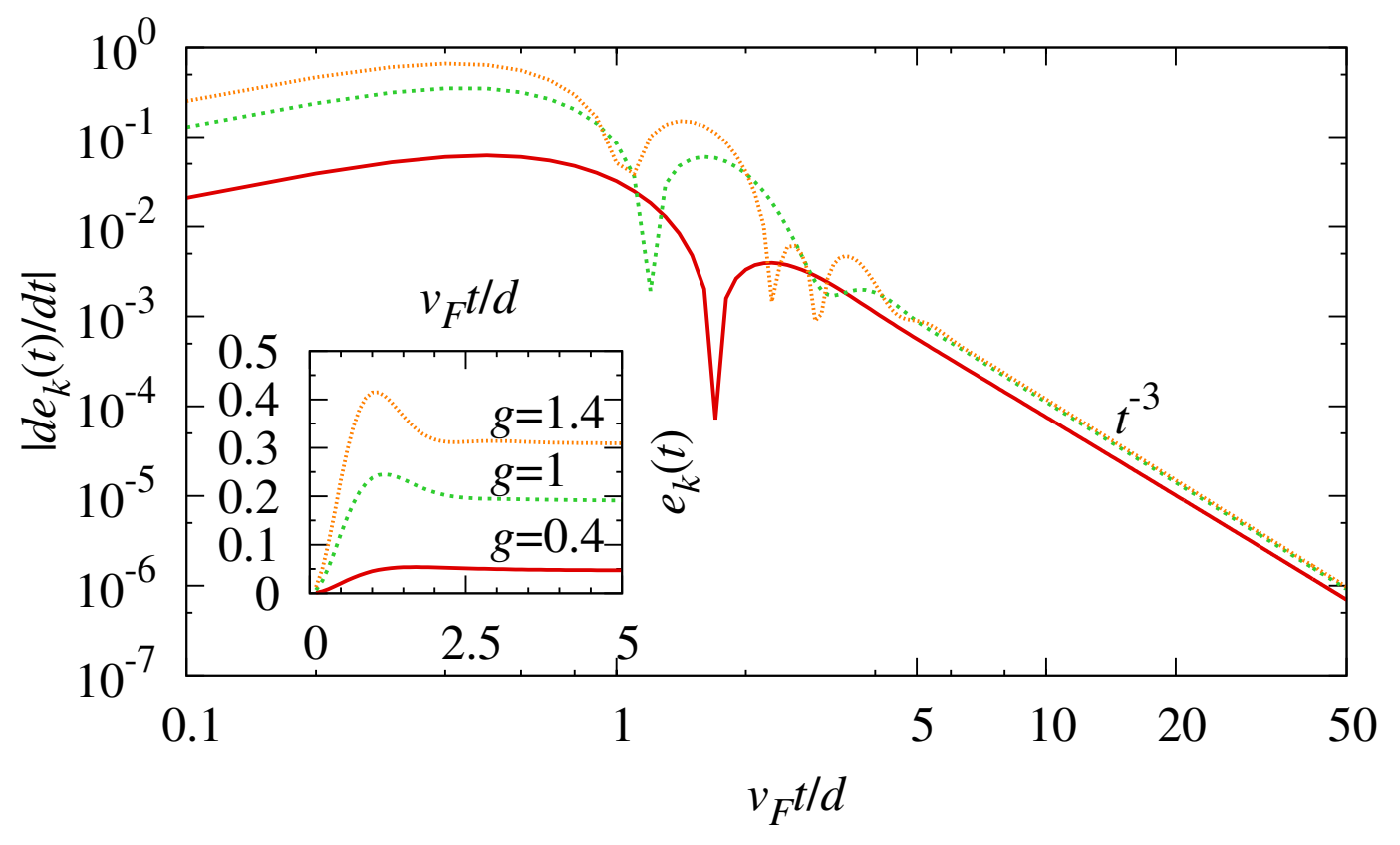

Figura 2.1: La derivada de la energía cinética por unidad de longitud para diferentes valores de la constante de acoplamiento $g$. En el inset puede observarse que la energía cinética por unidad de longitud alcanza un máximo y luego va a un estado estacionario. $e_{\text {kin }}(t)$ está en unidades de $\frac{v_{F}}{2 \pi d^{2}}$. 
matriz densidad de una partícula:

$$
C_{\psi_{r}}(x, \infty) \simeq C_{\psi_{r}}^{(i)}(x) e^{-\frac{g}{4} K_{i} \log ^{2}(x / d)} .
$$

Vemos entonces que el decaimiento principal viene dado por la corrección producida por el quench (y no por la porción debida a las condiciones iniciales que es una ley de potencias). La función de correlación en el estado estacionario decae más rápido que cualquier ley de potencias y en particular decae más rápido que en equilibrio, en donde decae como $e^{-c \log ^{3 / 2}(x / d)}$ [131]. Esto refleja un hecho general, a saber, en el estado estacionario que se alcanza luego de un quench global (como un quench de interacción) las correlaciones decaen más rápidamente que en el estado fundamental del Hamiltoniano $H_{f}$ que dicta la evolución temporal. Esto puede entenderse fácilmente dado que el quench inyecta una cantidad extensiva de energía al sistema (la cual puede calcularse como la diferencia entre la energía del estado inicial con respecto a $H_{f}$ y la energía del estado fundamental de $H_{f}$ ), lo que produce que el sistema se "caliente" y, por lo tanto, que las correlaciones decaigan más rápido.

Pasamos ahora a la descripción estadística del estado estacionario. En el caso del ML las cantidades conservadas son los números de ocupación $\hat{n}(q)=\alpha_{q}^{\dagger} \alpha_{q}$ de los modos bosónicos que diagonalizan $H_{f}$. El GGE puede interpretarse entonces como un estado en el que cada modo bosónico se encuentra a una temperatura diferente $\beta(q)=\lambda(q) / \epsilon_{f}(q)$ (ver Ec. (2.46) en el capítulo anterior). El estado final del sistema es, por lo tanto, no térmico, algo que podía anticiparse debido a la presencia de un gran número de cantidades conservadas. Por lo tanto, en modelos cuadráticos (o en aquellos que pueden mapearse a un modelo cuadrático) el único mecanismo de relajación es el dephasing y el estado final que alcanza el sistema como resultado de tal relajación es el GGE. Como se ha visto en este capítulo, la relajación por dephasing típicamente genera decaimientos sin una escala definida, tipo ley de potencias o logarítmicos que son característicos de la suma de una función sobre un espectro denso de frecuencias [3].

La validez del GGE para modelos cuadráticos, tal como está expresada en la Ec. 1.10), establece, en particular, que todas las funciones de correlación de tiempos iguales de un dado subsistema en el estado estacionario vienen determinadas por el GGE. La cuestión de las funciones de correlación dinámicas, es decir, de dos o más tiempos, es un poco más sutil. En lo que resta de esta sección argumentaremos en favor de que las funciones de correlación de dos tiempos, las cuales están relacionadas con las funciones respuesta del sistema mediante la teoría de respuesta lineal, también quedan determinas por el GGE. Para empezar, consideraremos como ejemplo el límite estacionario de la función de Green de dos tiempos:

$$
C_{\psi_{r}}\left(x, t, t_{0}\right)=\left\langle\psi_{r}^{\dagger}\left(x, t+t_{0}\right) \psi_{r}\left(0, t_{0}\right)\right\rangle,
$$

donde $\langle\ldots\rangle$ denota valores de expectación en el estado inicial, al cual tomaremos como un gas de Fermi no interactuante $\left(K_{i}=1\right)$ por simplicidad. Después de utilizar la identidad de 
bosonización, Ec. (2.16), y la fórmula de Baker-Hausdorff, obtenemos:

$$
C_{\psi_{r}}\left(x, t, t_{0}\right)=\frac{1}{2 \pi a} e^{\left[\phi_{r}\left(x, t+t_{0}\right), \phi_{r}\left(0, t_{0}\right)\right]}\left\langle e^{i\left(\phi_{r}\left(x, t+t_{0}\right)-\phi_{r}\left(0, t_{0}\right)\right)}\right\rangle,
$$

en donde el conmutador es una función a números complejos (y no un operador) que depende de $t$. Como estamos tratando con una teoría gaussiana podemos hacer uso de la propiedad $\left\langle e^{A}\right\rangle=e^{-\frac{1}{2}\left\langle A^{2}\right\rangle}$. La cantidad de interés es el exponente,

$$
\begin{aligned}
& \left\langle\left(\phi_{r}\left(x, t+t_{0}\right)-\phi_{r}\left(0, t_{0}\right)\right)^{2}\right\rangle= \\
& \sum_{q>0} \frac{e^{-a q}}{n_{q}}\left\{\left|e^{i q x} f\left(q, t+t_{0}\right)-f\left(q, t_{0}\right)\right|^{2}+\left|e^{-i q x} g\left(q, t+t_{0}\right)-g\left(q, t_{0}\right)\right|^{2}\right\},
\end{aligned}
$$

con las funciones $f g$ definidas como

$$
\begin{aligned}
& f(q, t)=\cos \left[\epsilon_{f}(q) t\right]-i \sin \left[\epsilon_{f}(q) t\right] \cosh \left[2 \theta_{f}(q)\right], \\
& g(q, t)=i \sin \left[\epsilon_{f}(q) t\right] \sinh \left[2 \theta_{f}(q)\right] .
\end{aligned}
$$

En el límite $t \rightarrow \infty$ los términos oscilantes se promedian a un número y obtenemos

$$
\begin{aligned}
& \lim _{t_{0} \rightarrow \infty}\left\langle\left(\phi_{r}\left(x, t+t_{0}\right)-\phi_{r}\left(0, t_{0}\right)\right)^{2}\right\rangle= \\
& \begin{aligned}
2 \sum_{q>0} \frac{e^{-a q}}{n_{q}} \times\left\{\cosh ^{2}\left(2 \theta_{f}(q)\right)\left(1-\cos (q x) \cos \left(\epsilon_{f}(q) t\right)\right)\right. \\
\left.-\cosh \left(2 \theta_{f}(q)\right) \sin (q x) \sin \left(\epsilon_{f}(q) t\right)\right\} .
\end{aligned}
\end{aligned}
$$

Ahora compararemos este resultado con el del GGE. En términos de la base bosónica $\left\{\alpha_{q}, \alpha_{q}^{\dagger}\right\}$ que diagonaliza el Hamiltoniano final, $H_{f}=\sum_{q} \epsilon_{f}(q) \alpha_{q}^{\dagger} \alpha_{q}$, la matriz densidad del GGE puede escribirse como

$$
\rho_{\mathrm{gG}}=\frac{1}{Z_{\mathrm{gG}}} \exp \left[\sum_{q>0} \lambda(q) \hat{n}(q)\right],
$$

donde $\hat{n}(q)=\alpha_{q}^{\dagger} \alpha_{q}, Z_{\mathrm{gG}}=\operatorname{Tr}\left[\exp \sum_{q>0} \lambda(q) \hat{n}(q)\right]$, y los multiplicadores de Lagrange $\lambda(q)$, fijados por las condiciones iniciales, adquieren la forma particular

$$
\lambda(q)=\langle\hat{n}(q)\rangle_{\mathrm{Gg}}=\langle\hat{n}(q)\rangle=\sinh ^{2}\left(\theta_{f}(q)\right)
$$

en donde $\langle\hat{O}\rangle_{\mathrm{Gg}}=\operatorname{Tr}\left[\rho_{\mathrm{Gg}} \hat{O}\right]$. Mostraremos que, si definimos $C_{\psi_{r}}^{\mathrm{gG}}(x, t) \equiv\left\langle\psi_{r}^{\dagger}(x, t) \psi_{r}(0,0)\right\rangle_{\mathrm{Gg}}$, entonces

$$
\lim _{t_{0} \rightarrow \infty} C_{\psi_{r}}\left(x, t, t_{0}\right)=C_{\psi_{r}}^{\mathrm{Gg}}(x, t)
$$

Para mostrarlo, procedemos análogamente a como lo hicimos en el cálculo anterior (fórmula 
de Baker-Hausdorff e identidad de valores medios gaussianos). Obtenemos el resultado del GGE,

$$
C_{\psi_{r}}^{\mathrm{gG}}(x, t)=\frac{1}{2 \pi a} e^{\left[\phi_{r}(x, t), \phi_{r}(0,0)\right]} \exp \left[-\frac{1}{2}\left\langle\left(\phi_{r}(x, t)-\phi_{r}(0,0)\right)^{2}\right\rangle_{\mathrm{Gg}}\right] .
$$

De nuevo, la parte crucial a comparar es el exponente, dado que los otros factores son iguales a los presentes en la Ec. (2.41). Todos los valores medios no nulos que sobreviven en el exponente de la Ec. (2.49) vienen dados por $\left\langle\alpha_{q}^{\dagger} \alpha_{q}\right\rangle_{\mathrm{Gg}}$ o por $\left\langle\alpha_{q} \alpha_{q}^{\dagger}\right\rangle_{\mathrm{Gg}}$, los cuales pueden obtenerse usando la Ec. (2.47). Utilizando este resultado, al desarrollar el exponente en Eq. (2.49) encontramos que es el mismo del cálculo dinámico Ec. (2.45). Destacamos que este resultado vale tanto para potenciales de corto alcance como para el potencial de Coulomb, para el cual, si bien la velocidad $v_{f}(q)$ tiene un divergencia a $q$ chico, la energía $\epsilon_{f}(q)$ es siempre finita, lo que asegura que haya dephasing, el prerrequisito esencial para la validez del cálculo.

Recientemente fue dada una prueba general para el hecho de que la misma matriz densidad que reproduce las funciones de correlación estáticas luego de la relajación puede describir funciones de correlación dinámicas. La prueba está basada en las cotas de Lieb-Robinson [85] para la velocidad de propagación de información en sistemas con interacciones de corto alcance. El hecho de que el cálculo que expusimos más arriba pueda ser llevado a cabo para un sistema con interacciones de Coulomb indica que la hipótesis de interacciones de corto alcance puede relajarse en algunos casos. Posiblemente esto esté relacionado con el hecho de que aunque no hay un límite finito para la velocidad de propagación de la información transportada por las CPs plasmónicas, de todos modos las correlaciones exhiben una especie de efecto horizonte (véase el siguiente apartado), propio de los modelos con interacciones de corto alcance.

\section{Efecto cono de luz}

Pasaremos ahora a discutir un fenómeno muy importante en la dinámica fuera de equilibrio de sistemas aislados, el efecto cono de luz o efecto horizonte. En sistemas con interacciones de corto alcance, las correlaciones espacio-temporales entre dos puntos separados por una distancia $x$ exhiben el efecto cono de luz: en la región del espacio tal que $x>2 v t$, donde $v$ es la velocidad característica de propagación de las excitaciones, las correlaciones mantienen esencialmente la misma forma que en el estado inicial. En la región complementaria, $t>$ $x / 2 v$, las correlaciones tienen la forma del estado asintótico de tiempos grandes (estado estacionario del sistema). La presencia de este efecto ha sido demostrada rigurosamente en el caso de un quench en sistemas unidimensionales con invariancia conforme [16], de los cuales el ML es un ejemplo específico. Este caso concreto puede entenderse de la siguiente manera: en estos modelos la relación de dispersión de las cuasipartículas (CPs), que en el caso del ML son los modos bosónicos que diagonalizan el Hamiltoniano, es lineal $\epsilon(q)=v q$, en donde $v=\frac{d \epsilon(q)}{d q}$ es la velocidad de grupo (aproximadamente constante) de las CPs. El 

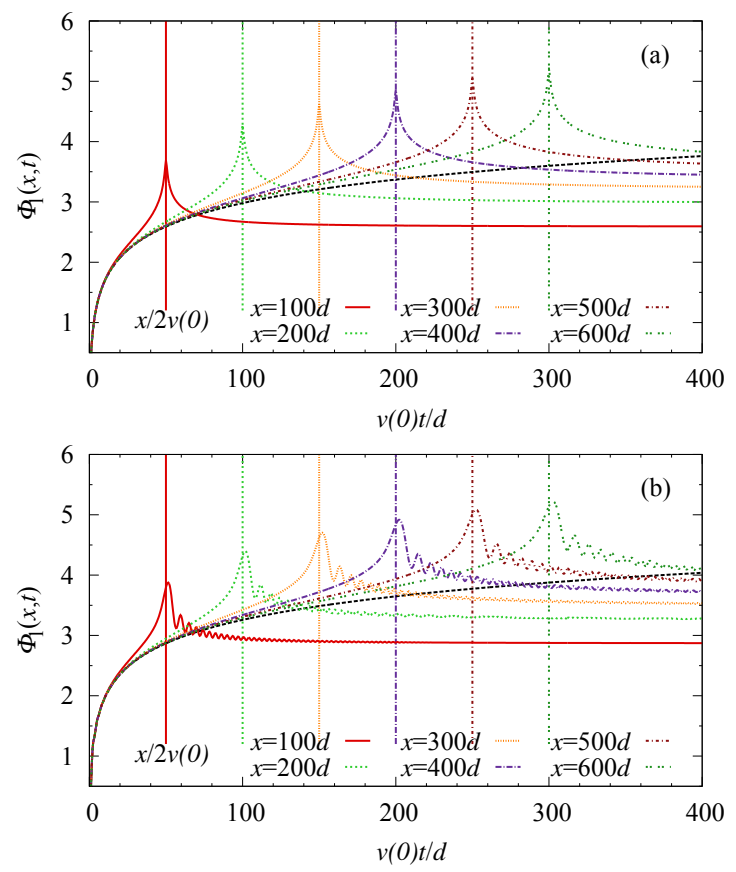

Figura 2.2: Evolución temporal de $\Phi_{1}(x, t)$ a partir de integración numérica para varios valores de $x$ luego de encender una interacción de corto alcance (a) usando la aproximación $a d$ hoc $\sinh 2 \theta_{q} \approx e^{-q R_{0}}\left(K_{f}-K_{f}^{-1}\right) / 2$ con $R_{0}=d$ y $K_{f}=0,5$ (b) considerando la dependencia en momento de un potencial gaussiano Ec. (2.54) $\left(\sigma^{2}=d^{-2}\right)$ con le mismo parámetro de Luttinger $K_{f}$. La línea negra discontínua es, en ambos gráficos, el resultado para $x \rightarrow \infty$. Las líneas verticales son, para cada $x$, los tiempos definidos en la Ec. (2.55).

estado inicial (un estado excitado muy complicado del Hamiltoniano $H_{F}$ ) actúa como una fuente de CPs que se propagan semiclásicamente a lo largo del sistema con velocidad $v$. Los pares de CPs que emergen desde el mismo punto están entrelazados y su llegada a dos puntos distantes afecta las correlaciones entre ellos. Esta imagen simple ha sido validada en una serie de trabajos teóricos [30, 67, 68, 36, 37, 2, 82] e incluso experimentalmente [33]. Más adelante ejemplificaremos este efecto en el caso del ML con interacciones de corto alcance. Es interesante, sin embargo, considerar el fenómeno de la propagación de información en condiciones fuera de equilibrio para el caso de un sistema unidimensional con interacciones de Coulomb, en particular, el ML. En tal caso cabe notar que la interpretación simple descripta más arriba se enfrenta con un problema, dado que la velocidad de grupo $v_{f}(q)$ diverge cuando $q \rightarrow 0$ mientras que la energía es finita. Además, la distribución de momentos inicial de las $\mathrm{CPs}\left\langle\alpha_{q}^{\dagger} \alpha_{q}\right\rangle=\sinh ^{2}\left(\theta_{f}(q)\right)$, la cual es una constante de movimiento, está picada alrededor de $q=0$, la región en donde la velocidad varía más abruptamente. Por lo tanto, basándonos en la imagen semiclásica propuesta antes, deberíamos concluir que las correlaciones del estado estacionario se propagan instantáneamente a través del sistema. Veremos que este no es el caso y señalaremos cual es la aproximación que falla en el esquema anterior en el caso de que haya interacciones de largo alcance. 
Primero mostraremos algunos resultados analíticos en los regímenes asimtóticos. Comenzaremos por analizar la integral que define a la función $\Phi_{1}(x, t)$ en Ec. (2.32) (el análisis es completamente análogo para $\left.\Phi_{2}(x, t)\right)$ dado que esta integral define la corrección inducida por el quench a las correlaciones en el estado inicial $C_{\psi_{r}}^{(i)}(x)$. después de integrar por partes encontramos que para $x \gg d$ y para $t$ fijo

$$
\Phi_{1}(x, t) \simeq 2 \log \left(d x^{-1}\right) \sinh ^{2}\left(2 \theta_{f}\left(x^{-1}\right)\right) \sin ^{2}\left(\epsilon_{f}\left(x^{-1}\right) t\right)+\xi(t),
$$

en donde

$$
\xi(t)=2 \int_{0}^{\infty} \frac{d q}{q} \sinh ^{2}\left(2 \theta_{f}(q)\right) \sin ^{2}\left(\epsilon_{f}(q) t\right) \simeq-\frac{g}{2} \log ^{2}\left(2 v_{F} t / d\right)
$$

es una función que sólo depende de $t$. la última igualdad es válida si $v_{F} t \gg d^{2}$. El primer término en Ec. (2.50) va a cero aproximadamente como $-\frac{\left(\nu_{F} t\right)^{2}}{x^{2}}$ cuando $2 \epsilon_{f}\left(x^{-1}\right) t \ll 1$, y por lo tanto representa una corrección subdominante con respecto a las correlaciones del estado inicial, Ec. (2.34). Por lo tanto, la corrección inducida por el quench en la matriz densidad de una partícula se reduce, a menos de correcciones exponencialmente pequeñas, a un prefactor dependiente del tiempo:

$$
C_{\psi_{r}}(x, t)=C_{\psi_{r}}^{(i)}(x) e^{-\frac{g}{4} K_{i} \log ^{2}\left(2 \nu_{F} t / d\right)},
$$

para tiempos tales que

$$
\frac{d}{v_{F}} \ll t \ll \tilde{t}_{x}=\frac{x}{2 v_{F} \sqrt{1-2 g \log \left(d x^{-1}\right)}} .
$$

En la última ecuación hemos usado la forma explícita de la dispersión plasmónica Ec. (2.37), y consideramos distancias $x \gg d$. Para tal régimen de tiempos cortos, las correlaciones tienen la misma forma que en el estado inicial, es decir, vienen dadas por $C_{\psi_{r}}^{(i)}(x)$.

Notemos que el denominador en la Ec. (2.53) es esencialmente la velocidad de grupo $2 v_{f}\left(x^{-1}\right)$ y por lo tanto podemos escribir la Ec. (2.53) como $\frac{x}{2 v_{f}\left(x^{-1}\right)} \gg t$. Para un potencial de corto alcance la velocidad de grupo $v_{f}(q)$ tiende a una constante $v_{f}(0)$ cuando $q \rightarrow 0$, lo que determina un cono de luz lineal $\frac{x}{2 v(0)}=t$ para $x$ suficientemente grande. Por el contrario, la divergencia (logarítmica) en $q \rightarrow 0$ del potencial de largo alcance genera un cono de luz ligeramente no lineal, como sugiere la Ec. (2.53), en el cual el comportamiento de las funciones de correlación cambian de un régimen de tiempos cortos a uno de tiempos largos. Este resultado puede entenderse en términos del siguiente argumento: las peculiaridades de la relación de dispersión plasmónica en 1D se reflejan en que al disminuir $q$, la energía de los modos plasmónicos decrece mientras que su velocidad de grupo diverge. Consecuentemente, y dado que un modo con momento $q$ sólo puede propagar correlaciones (información) a lo largo de distancias mayores que $1 /$ q (su longitud de onda), la distancia mínima a partir de la cual los modos más rápidos pueden propagar información es mayor que para los modos más

\footnotetext{
${ }^{2}$ For $v_{F} t \ll d, \xi(t)$ grows as $t^{2}$.
} 
lentos. En otras palabras, a tiempos cortos sólo los modos con altas energías están activos, pero éstos tienen una velocidad pequeña y por lo tanto no pueden propagarse rápido. A tiempos más largos, se van activando los modos con energías más bajas, cuyas velocidad es cada vez más grande. Esta explicación está en acuerdo con la Ec. (2.53). Por lo tanto, las interacciones de largo alcance revelan explícitamente el carácter cuántico de la propagación de las CPs. El carácter sublineal del cono de luz implica que la información puede propagarse más rápido que cualquier velocidad del sonido definida (sólo debemos esperar suficiente tiempo para que la propagación de las correlaciones sea más rápida que una dada velocidad). Sin embargo, este efecto no se debe a una aceleración de las excitaciones, dado que cada modo tiene una velocidad constante $v(q)$, sino a la naturaleza cuántica de las excitaciones colectivas, que hace que los modos cada vez más rápidos sean relevantes sólo a tiempos cada vez más grandes. Si este efecto está presente in modelos 1D con interacciones de largo alcance en la red es un problema abierto. El hecho de que esté relacionado con las peculiaridades de la dispersión plasmónica en $1 \mathrm{D}$ sugieren su generalidad. En tal caso, y dado que las desviaciones de la linealidad no son fuertes sino una corrección logarítmica, las simulaciones con algoritmos actuales debería ser posible.

Casos de efecto horizonte no lineales han sido reportados también en sistemas con interacciones de corto alcance. Sin embargo, los mecanismos causantes de tal fenómeno son totalmente diferentes al descripto antes. Por ejemplo, se ha detectado cierto tipo de modelos bosónicos para los cuales el cono de luz está "doblado hacia fuera" (hay una aceleración en la transmisión de la información) [41]. También, se ha hallado que en cadenas de espines con desorden el horizonte puede exhibir un crecimiento logarítmico con el tiempo, es decir estar "doblado hacia dentro" $[13,14]$.

Para complementar la discusión anterior analizaremos el efecto horizonte en el caso de un quench en el que se enciende repentinamente un potencial de interacción de corto alcance y lo compararemos con el quench de interacción de Coulomb. En la Fig. 2.2 mostramos la dependencia temporal de $\Phi_{1}(x, t)$ para diferentes valores de $x$ usando la prescripción ad hoc detallada en la Ec. (2.28) junto con los resultados obtenidos al considerar la dependencia en momento completa de un potencial Gaussiano

$$
V(q)=V(0) \exp \left[-\frac{q^{2}}{2 \sigma^{2}}\right]
$$

Para que la comparación tenga sentido hemos elegido los potenciales de tal manera que el parámetro de Luttinger $K_{f}$ y la velocidad $v(q=0)$ sean los mismos en ambos casos. Para los dos casos, observamos que a tiempos cortos las correlaciones del estado inicial dominan y la función de correlación sigue la curva correspondiente a $x \rightarrow \infty$. Luego la función de correlación se desvía de tal comportamiento asintótico, desarrolla un máximo y luego decae a un valor constante, lo que señala el establecimiento de un régimen estacionario. Las diferencias entre los resultados para los dos potenciales de corto alcance también son interesantes. Para 


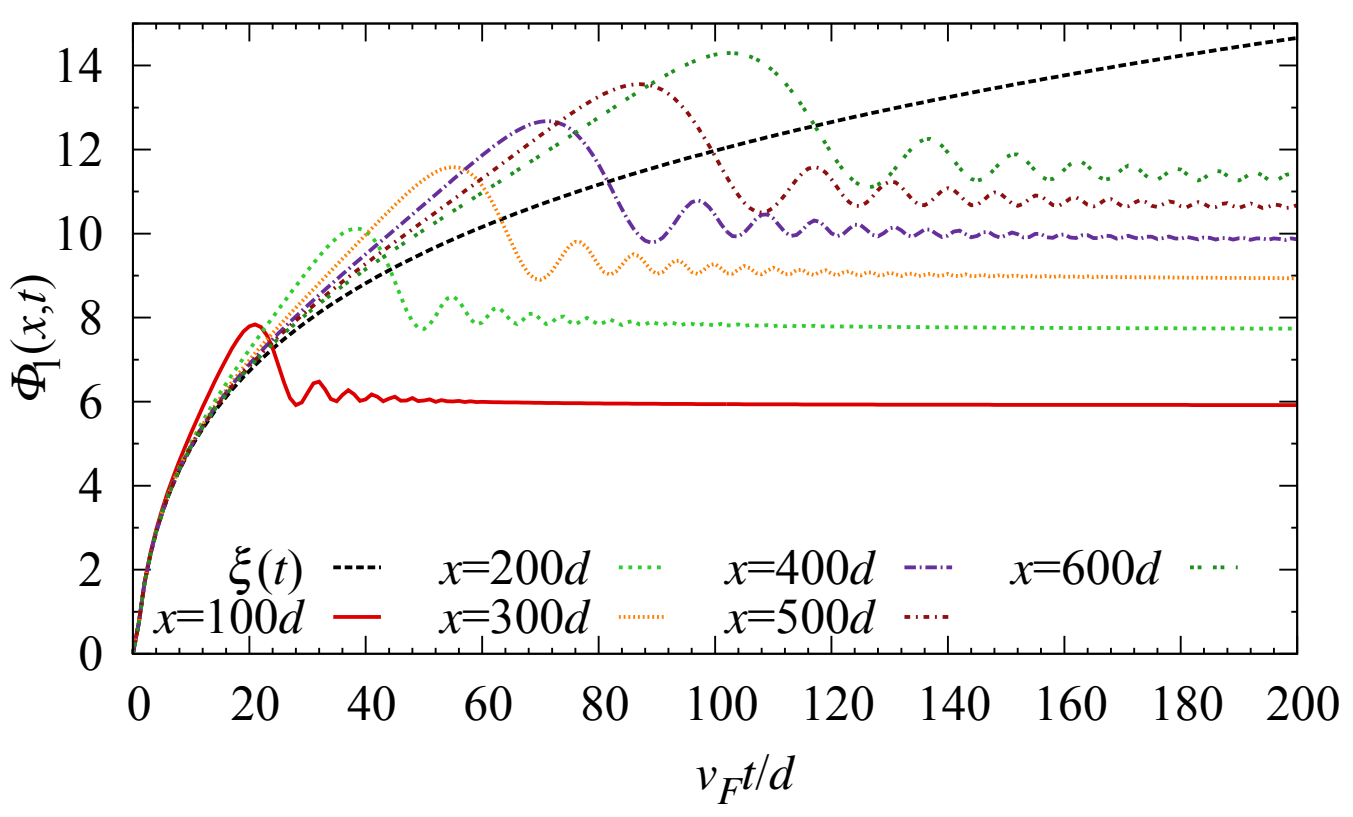

Figura 2.3: Evolución temporal de $\Phi_{1}(x, t)$ para varios valores de $x$ luego de un quench desde un estado inicial con interaccions de corto alcance a interaccioens de corto alcance con $g=1$. La línea discontínua representa la función $\xi(t)$ definida en la Ec. (2.51).

la aproximación ad hoc el máximo es un pico pronunciado localizado en

$$
t=\frac{x}{2 v(0)} .
$$

Por otro lado, al considerar la dependencia en momento completa del potencial Gaussiano, el pico se ensancha y aparecen oscilaciones montadas sobre el decaimiento final a la constante. La posición de los máximos queda descripta sólo aproximadamente por la Ec. (2.55). Esto ilustra la discusión anterior, de acuerdo con la cual un potencial de corto alcance está relacionado con un horizonte lineal en virtud de la finitud del potencial en $q=0$. Además, podemos concluir que una dispersión finita en la distribución de velocidades de las CPs que transportan las nuevas correlaciones induce oscilaciones en la fucnión de correlación y ensancha la región de transición en la cual las correlaciones cambian su comportamiento.

Para el potencial Coulombiano, Fig. (2.3), emergen algunas características similares, aunque el pico en este caso es apreciablemente más ancho que en el caso del potencial Gaussiano dado que en este caso la velocidad de los plasmones varía a lo largo de un conjunto no acotado de valores. Existe otra diferencia mucho más interesante: los máximos no se encuentran en una líne recta en el plano espacio-temporal (como era el caso en los ejemplos anteriores. Esto se muestra en la Fig. (2.4). En la región $I$ las correlaciones tienen el mismo comportamiento con $x$ que en el estado inicial y el efecto del quench se refleja sólo en un prefactor que depende del tiempo, Ec. (2.52), mientras que en la región II dominan las correlaciones del estado 


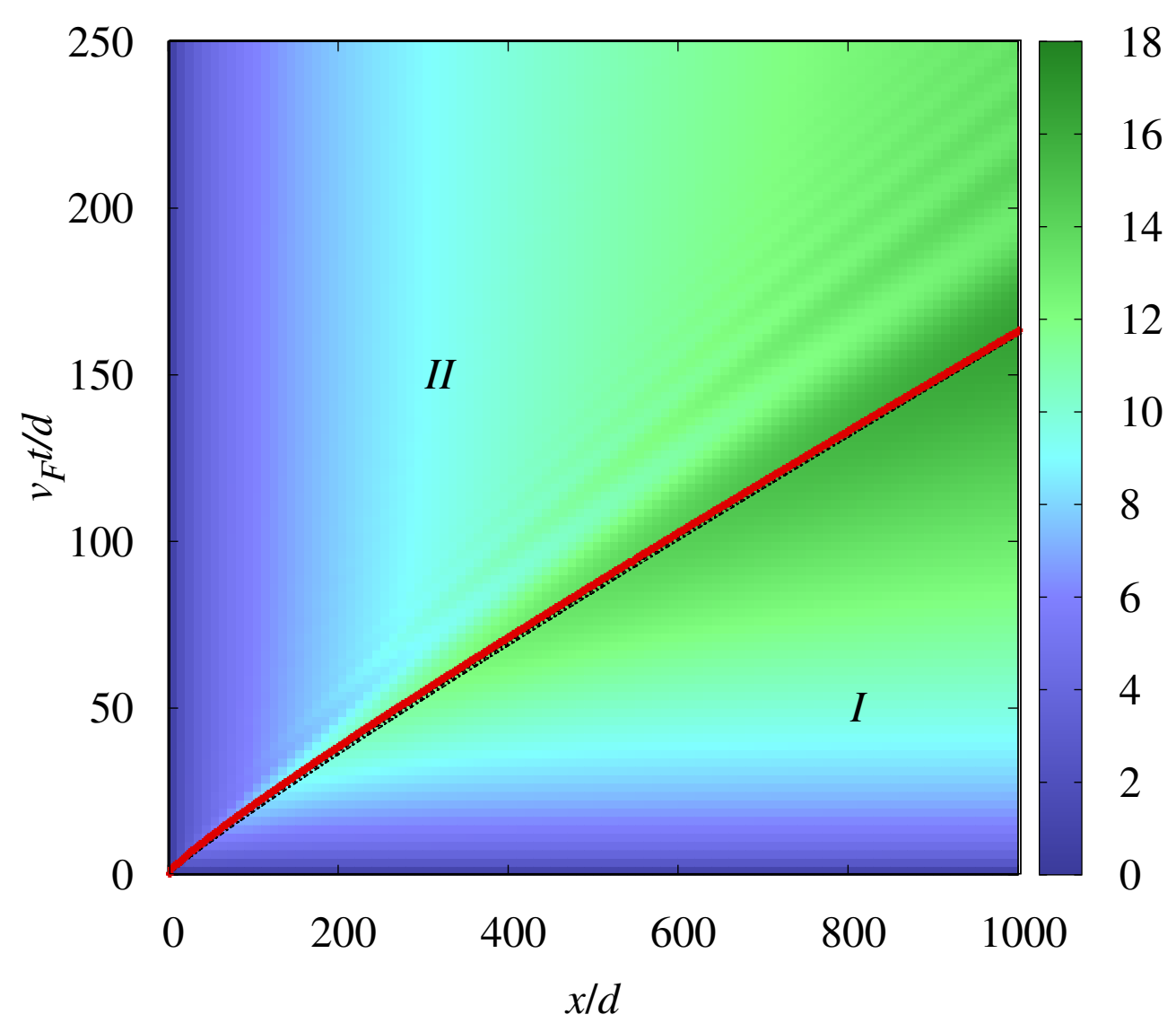

Figura 2.4: Gráfico de color falso de la función $\Phi_{1}(x, t)$ para $g=1$. La línea discontínua negra es la curva $t=1,26 \tilde{t}_{x}$ (Ec. 2.53). La línea roja sólida marca las posiciones d elos máximos. En la región I dominan las correlaciones del estado inicial. En la región II dominan las correlaciones del estado estacionario. 
estacionario, Ec. (2.39). Entre las regiones $I$ y $I I$ existe una amplia región de transición en donde la función alcanza un máximo y oscila. El centro de la región de transición queda bien estimado por la posición de los máximos, que, a su vez, coincide con la curva $t=1,26 \tilde{t}_{x}$ (Ec. 2.53). Las desviaciones de la linealidad son débiles, dado que están determinadas por un factor $\log ^{1 / 2} x / d$.

Finalmente, notamos que se ha demostrado que la dinámica de las CPs está íntimamente relacionada con el ritmo de crecimiento de la entropía de entanglemente después del quench. Por ejemplo, en una teoría conforme, la velocidad de propagación bien definida de las CPs está relacionada con el crecimiento lineal en el tiempo de la entropía de entanglement luego de un quench global [15]. Por lo tanto, podemos especular que la entropia de entanglement luego de un quench en el ML con interacciones de Coulomb exhibirá alguna especie de crecimiento no lineal debido a la no linealidad del cono de luz.

\subsection{Relevancia del ML para la dinámica de modelos microscópicos}

Consideremos el siguiente modelo en la red $H=H_{0}+H_{1}$ de fermiones sin espín con interacciones y hopping a primeros vecinos:

$$
\begin{aligned}
& H_{0}=-J \sum_{j} c_{j}^{\dagger} c_{j+1}+c_{j+1}^{\dagger} c_{j}, \\
& H_{1}=\Delta \sum_{j} n_{j} n_{j+1},
\end{aligned}
$$

en donde $c_{j}^{\dagger}$ crea un fermión en el sitio $j$ de una cadena de $L$ sitios y $n_{j}=c_{j}^{\dagger} c_{j}$. Asumimos condiciones de contorno periódicas, $c_{L+m}^{\#}=c_{m}^{\#}$. Aunque no vayamos a explotar esta característica, cabe aclarar que este modelo es integrable por Bethe ansatz y puede mapearse mediante una transformación de Jordan-Wigner al modelo XXZ. Para $\Delta<2 J$ la física de equilibrio de este modelo, es decir, sus propiedades de baja energía, pueden capturarse mediante el paradigma del líquido de Luttinger. En otras palabras, el ML es el punto fijo infrarrojo del modelo Ec. (2.56). Eso significa que el comportamiento de largas distancias de todas las funciones de correlación del modelo de la Ec. Ec. (2.56) pueden calcularse mediante el ML a través de la fórmula de bosonización de los operadores fermiónicos, Ec.(2.16), utilizando los valores adecuados de las constantes $K$ y $v$ que serán ciertas funciones de $J$ y $\Delta$ y el llenado. Para obtener esos valores es necesario "bosonizar" el Hamiltoniano Ec. (2.56), es decir, tomar el límite contínuo (el espaciado de red yendo a cero) y encontrar aquellos términos en el Hamiltoniano cinético y en el de interacción que son más relevantes en el lenguaje del grupo de renormalización. Para $\Delta<2 J$ el modelo resultante resulta idéntico al ML, con la particularidad de que las constantes de acoplamiento $g_{2}$ y $g_{4}$ vienen determinadas por los parámetros "microscópicos" presentes en el Hamiltoniano Ec. (2.56). A primer orden en la constante de interacción $\Delta$ los parámetros de Luttinger del modelo Ec. (2.56) son: 


$$
\begin{aligned}
K & =\sqrt{\frac{v_{F}-\Delta}{v_{F}+3 \Delta}}, \\
\nu & =\frac{1}{\pi} \sqrt{\left(\pi v_{F}+\Delta\right)^{2}-4 \Delta^{2}},
\end{aligned}
$$

en donde $v_{F}=\left.\frac{\partial \epsilon(k)}{\partial k}\right|_{k=k_{F}}$, $\operatorname{con} \epsilon(k)=-t \cos (2 k \pi / L)$, es la velocidad de Fermi.

Ahora podemos hacernos la siguiente pregunta: ¿la dinámica de no equilibrio del modelo definido por la Ec. (2.56) (por ejemplo, después de un quench) tendrá alguna relación con la dinámica de no equilibrio del ML? En principio la respuesta es no, ya que los operadores que son irrelevantes para la física de equilibrio bien pueden ser relevantes fuera de equilibrio ya que el sistema, en tales condiciones, explora regiones del expectro de excitaciones que pueden estar muy lejos del estado fundamental. Pondremos, sin embargo, un poco de esfuerzo en contestar esta pregunta más detalladamente y mostraremos que al menos para tiempos moderadamente cortos, el ML captura ciertas propiedades de baja energía fuera de equilibrio del modelo mictroscópico. Para ellos calcularemos la dinámica después de un quench del modelo Ec. (2.56) y la compararemos con las predicciones del ML que han sido expuestas en las secciones anteriores.

Estudiaremos el sistema a llenado medio $\left(k_{F}=\frac{\pi}{2}\right)$ y consideraremos como estado inicial el estado fundamental del Hamiltoniano con $\Delta=0$, es decir, un gas de fermiones libres. De esta manera, estaremos realizando un quench de interacción (de corto alcance) como los que hemos estudiado en el caso del ML. Resolver la dinámica de no equilibrio de un modelo interactuante de muchos cuerpos como el Hamiltoniano Ec. (2.56) es una tarea muy difícil. Entre las diferentes alternativas a disposición, la utilización de diferentes tipos de ecuaciones de evolución para correlaciones de pocos cuerpos son ampliamente utilizadas en la literatura actual (ver, por ejemplo [136, 46, 135, 6]). Parte del aporte original de esta tesis es el desarrollo de ciertas herramientas que permiten deducir tales ecuaciones de evolución partiendo de primeros principios. Esto será descripto en detalle en el Capítulo 4, sin embargo, para nuestros fines inmediatos nos limitaremos a decir que, en el caso del quench de interacción descripto antes, es posible estudiar la dinámica del modelo Ec. (2.56) usando una ecuación de evolución perturbativa en el parámetro $\alpha=\Delta / J$.

En la Fig. 2.5 mostramos la dinámica del modelo definido en la Ec. (2.56) (tal y como es capturada por nuestra ecuación de evolución) y las correspondientes predicciones del ML. Para la comparación usamos las cantidades $Z(t)$ y $e_{k i n}(t)$, cuyo comportamientos en el caso de un quench de itneracción de corto alcance vienen dados por las Ecs. (2.36) y (2.29) respectivamente. Para $Z(t)$ encontramos que, luego de un decaimiento Gaussiano a tiempos cortos (el cual también es exhibido por el ML para tiempos suficientemente cortos), el decaimiento a tiempos intermedios queda bien descripto por una ley de potencias cuyo exponente viene dado precisamente por las predicciones del ML. Para tiempos más largos, más allá de una escala de tiempos $t^{*}$ que depende de $\alpha$, se observan desviaciones. Estas desviaciones serán 

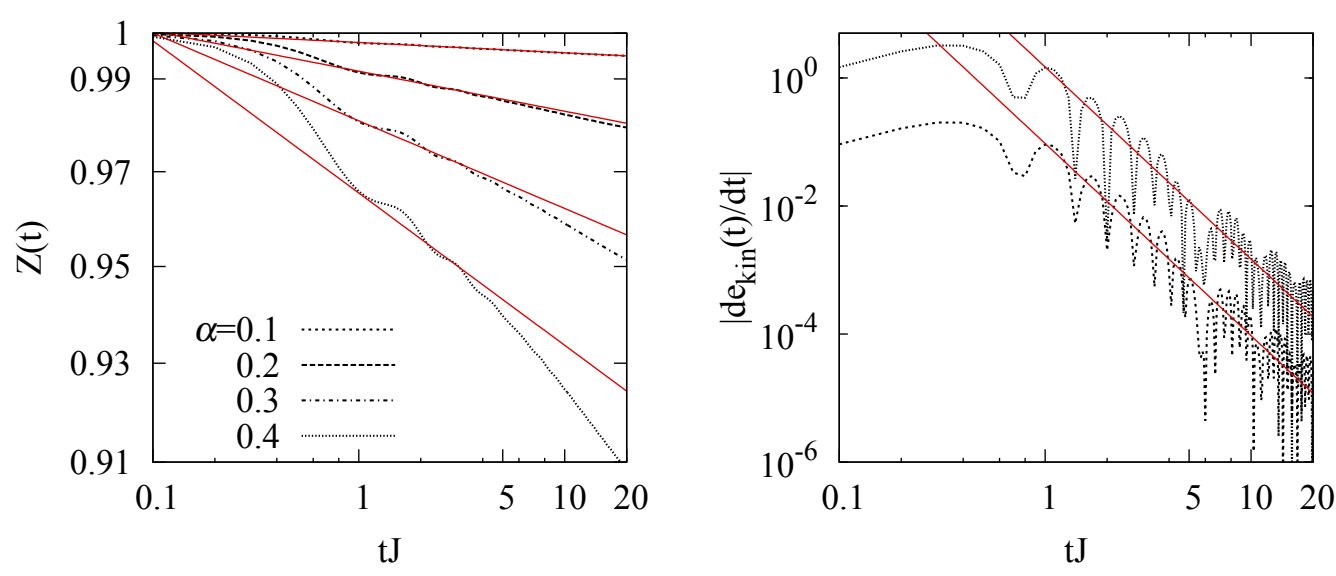

Figura 2.5: Panel derecho: Discontinuidad en la distribución de momentos en el momento de Fermi, $Z(t)$. Las líneas sólidas son leyes de potencias con el exponente dado por las predicciones del ML $Z(t) \sim t^{-\gamma}$, con $\gamma=\frac{1}{4}\left(K^{2}+K^{-2}-2\right)$. El parámetro de Luttinger se obtiene con bosonización del modelo en la red, $K=\sqrt{\frac{\pi v_{F}-\alpha}{\pi v_{F}+3 \alpha}}$. Panel izquierdo: Derivada de la energía cinética $e_{k i n}(t)$. las líneas sólidas son leyes de potencias $t^{-3}$ dadas por las predicciones del ML. El tamaño del sistema es $L=256$.

estudiadas en detalle en el Capítulo 5. Para $e_{k i n}(t)$ encontramos que las predicciones del ML funcionan bien incluso más allá de la escala de tiempos $t^{*}$ asociada con el $Z(t)$. Nótese que en la Fig. 2.5 no se ha realizado ningún ajuste de las curvas numéricas, sino que se ha mostrado, en conjunto con éstas, el resultado de una ley de potencia con los exponentes obtenidos de las Ecs. 2.58. Estos valores de los exponentes son sólo válidos al orden más bajo en $\Delta$, pudiéndose obtener expresiones a todo orden por medio del ansatz de Bethe [51]. Dado que la técnica que empleamos es intrínsecamente perturbativa, no es necesario acudir a estas expresiones exactas, las perturbativas son suficientes para nuestros fines.

El hecho de que el ML captura bien la dinámica de tiempos cortos después de un quench en ciertos sistemas en la red fue advertido por primera vez en la Ref. [74] en donde se realizó una comparación de los resultados obtenidos con t-DMRG y las predicciones del ML. Sin embargo, el t-DMRG provee acceso sólo a los momentos iniciales de la dinámica, por lo que en Ref. [74] no pudieron apreciarse las desviaciones a tiempos más largos. En la Ref. [60] se corroboró este hecho usando una técnica semianalítica que provee acceso a la dinámica de tiempos cortos. Es interesante que en ese mismo trabajo se demostró que los parámetros de Luttinger $K$ y $v$ que deben utilizarse para capturar la dinámica no son los parámetros renormalizados que describen la física de equilibrio, sino los parámetros que se obtienen de bosonizar perturbativamente el Hamiltoniano desnudo del modelo en cuestión. En el capítulo que sigue mostraremos, a partir de razonamientos analíticos, por qué la dinámica del LM captura las primeras etapas de la relajación de modelos más realistas y por qué necesariamente los parámetros que intervienen están relacionados con el Hamiltoniano microscópico del 
sistema y no con las propiedades de equilibrio de bajas energías del mismo.

En conclusión, existe evidencia de que el ML puede describir exitosamente la dinámica de no equilibrio de ciertos modelos que en equilibrio caen en su clase de universalidad. Sin embargo, para ciertas cantidades, las predicciones del ML parecen fallar más allá de una cierta escala de tiempos que se hace más y más corta al ir aumentando la intensidad de la interacción. Para cantidades como $e_{k i n}(t)$, que resultan de la suma de contribuciones provenientes desde varios modos de momento $\left(e_{k i n}(t)=\sum_{k} \epsilon(k) n(k, t)\right)$ las predicciones del ML parecen tener validez en rangos temporales más grandes. En un contexto más amplio, podemos señalar que esto está indicando que la relajación de los sistemas interactuantes se da en varias etapas y que la primera de ellas está dominada por el dephasing. Abordaremos en detalle este tema en el siguiente capítulo. 



\section{Pretermalización}

En el capítulo anterior hemos visto que para un modelo cuadrático el único mecanismo disponible de relajación luego de un quench global es el dephasing entre los diferentes modos libres. También quedó claro que los estados estacionarios que surgen luego de tal relajación son altamente no térmicos, pero que aun así pueden ser descriptos mediante un tipo especial de ensamble estadístico, el GGE. En este capítulo mostraremos que estos resultados son relevantes también para sistemas más complejos en los que hay interacciones entre las partículas, es decir, que existe una especie de universalidad en la física del dephasing y el GGE. Para ilustrar esto utilizaremos otro modelo paradigmático, el líquido de Fermi (LF).

En equilibrio, la descripción por bosonización de un LF en $D>1$ está bastante bien establecida y, de hecho, puede obtenerse como la cuantificación de la teoría semiclásica de Landau [65, $64,63,26,25,27,80$ ]. Es decir, es posible describir un LF en equilibrio como un conjunto de modos bosónicos no interactuantes, los cuales representan las fluctuaciones en la forma de la superficie de Fermi [26, 25, 27]. En este capítulo mostraremos que a tiempos cortos luego de un quench de interacción lo suficientemente débil, la dinámica de un gas de Fermi también puede capturarse con un modelo de bosones no interactuantes. Es decir, a tiempos lo suficientemente cortos la dinámica del sistema puede describirse con un modelo efectivo representado por una colección de modos libres, el cual posee un número extensivo de cantidades conservadas, lo que implica que todos los conceptos estudiados en el capítulo anterior y enumerados antes son capaces de describir esa primera etapa de la relajación. En particular, el sistema exhibirá un estado pretermalizado que podrá ser descripto en términos de un GGE cuyas cantidades conservadas serán los números de ocupación de los modos bosónicos. En escalas de tiempo mayores, las interacciones entre los modos bosónicos (que no son tenidas en cuenta en el modelo efectivo pero que ciertamente están incluídas en el modelo original) rompen estas simetrías efectivas y llevan al sistema al equilibrio térmico final. Una de las ventajas de la descripción de bosonización es que pone en pie de igualdad los sistemas en $D=1$ y $D>1$ los cuales, fenomenológimente, están descriptos por paradigmas muy diferentes. De esta manera, el análisis del presente capítulo puede servir para justificar por qué las predicciones del modelo de Luttinger funcionan para sistemas más complicados en 
$D=1$, un hecho expuesto en el capítulo anterior pero que fue aceptado sin mayor justificación.

Como fue explicado en la Introducción de la tesis, la existencia de estados estacionarios de tiempos cortos que surgen como resultado de la dinámica de dephasing es llamada pretermalización en la literatura reciente. Tales estados metaestables han sido observados en una variedad de sistemas [95, 96, 40, 89, 90, 94, 140, 91, 43] y en el LF en particular en [95, 96, 40]. Al final del capítulo discutiremos algunas evidencias experimentales de la existencia de estos estados metaestables y también algunas perspectivas teóricas (complementarias a la presentada en este capítulo) sobre su naturaleza.

Los aportes originales correspondientes a este capítulo han sido publicados en Ref. [104].

\subsection{Análisis perturbativo de un quench de interacción en un gas de Fermi}

Para motivar la discusión, primero analizaremos perturbativamente la dinámica luego de encender repentinamente las interacciones en un gas de fermiones en $D>1$. Consideraremos un quench en la interacción en un gas de fermiones sin espín en $D=2$ (aunque los resultados pueden generalizarse fácilmente a un sistema en $D=3$ ). La dinámica del sistema para $t>0$ es dictada por el Hamiltoniano con interacciones de dos cuerpos $H=H_{0}+H_{\text {int }}$ con

$$
\begin{aligned}
H_{0} & =\sum_{\mathbf{k}} \epsilon(\mathbf{k}) c_{\mathbf{k}}^{\dagger} c_{\mathbf{k}}, \\
H_{\text {int }} & =\frac{1}{V} \sum_{\mathbf{k}_{1}, \mathbf{k}_{2}, \mathbf{q}} f(q) Q_{\mathbf{k}_{1}}(\mathbf{q}) Q_{\mathbf{k}_{2}}(-\mathbf{q}),
\end{aligned}
$$

en donde $c_{\mathbf{k}}, c_{\mathbf{k}}^{\dagger}$ son los operadores de campo fermiónicos, $\epsilon(\mathbf{k})$ es la relación de dispersión, $f(q)$ es la interacción de a pares (isotrópica) que se enciende a $t=0$ y $Q_{\mathbf{k}}(\mathbf{q})=c_{\mathbf{k}+\mathbf{q}}^{\dagger} c_{\mathbf{k}}$ crea excitaciones tipo p-h. La condición inicial es el estado fundamental $\left|\Psi_{0}\right\rangle$ de $H_{0}$, es decir, un mar de Fermi con todos los estados ocupados hasta el momento $k_{F}=\sqrt{4 \pi \rho_{0}}$, en donde $\rho_{0}=\mathscr{N} / V$ es la densidad media de fermiones. Asumimos que la interacción es de largo alcance: $f(q)=f_{0} F(q)$, en donde $f_{0}$ es pequeña comparada con la energía de Fermi y $F(q)$ decae rápidamente para $q \gg q_{c}$, en donde $q_{c}^{-1} \gg k_{F}^{-1}$ es el rango espacial de la interacción. Tales características del potencial son cruciales para poder fundamentar apropiadamente el tratamiento con bosonización que haremos más adelante. Sin embargo, antes de embarcarnos en esa tarea, haremos un análisis del quench usando teoría de perturbaciones dependiente del tiempo usando el lenguaje de los fermiones, de manera de obtener algunos resultados que podamos luego contrastar con la bosonización.

A nivel perturbativo en $f_{0}$, la dinámica de tiempos cortos de la distribución de momentos 
$n(\mathbf{k}, t)=\left\langle\Psi_{0}\left|e^{i H t} c_{\mathbf{k}}^{\dagger} c_{\mathbf{k}} e^{-i H t}\right| \Psi_{0}\right\rangle$ luego del quench viene dada por

$$
\begin{aligned}
n(\mathbf{k}, t) & =n(\mathbf{k}, 0)-\frac{8 f_{0}^{2}}{V^{2}} \sum_{\mathbf{k}^{\prime}, \mathbf{q}}\left|F\left(\mathbf{k}^{\prime}-\mathbf{k}+\mathbf{q}\right)-F(\mathbf{q})\right|^{2} \frac{\sin ^{2}\left[\frac{t}{2}\left(\epsilon(\mathbf{k})+\epsilon\left(\mathbf{k}^{\prime}\right)-\epsilon(\mathbf{k}+\mathbf{q})-\epsilon\left(\mathbf{k}^{\prime}-\mathbf{q}\right)\right)\right]}{\left(\epsilon(\mathbf{k})+\epsilon\left(\mathbf{k}^{\prime}\right)-\epsilon(\mathbf{k}+\mathbf{q})-\epsilon\left(\mathbf{k}^{\prime}-\mathbf{q}\right)\right)^{2}} \\
& \times\left[n(\mathbf{k}, 0) n\left(\mathbf{k}^{\prime}, 0\right)(1-n(\mathbf{k}+\mathbf{q}, 0))\left(1-n\left(\mathbf{k}^{\prime}-\mathbf{q}, 0\right)\right)-\right. \\
& \left.n(\mathbf{k}+\mathbf{q}, 0) n\left(\mathbf{k}^{\prime}-\mathbf{q}, 0\right)(1-n(\mathbf{k}, 0))\left(1-n\left(\mathbf{k}^{\prime}, 0\right)\right)\right]+\mathscr{O}\left(f_{0}^{3}\right) .
\end{aligned}
$$

Esta fórmula puede deducirse utiliando teoría de perturbaciones de segundo orden alrededor del estado inicial pero también puede obtenerse como el límite de tiempos cortos de una ecuación de evolución para la distribución de momentos más complicada que deduciremos en el Capítulo 4. Así que por ahora la aceptaremos sin más deducciones.

Al igual que en $D=1$ en el caso presente resulta conveniente analizar la dinámica del salto en la distribución de momentos en el nivel de Fermi, $Z^{\text {neq }}(t)=\left(\lim _{k \rightarrow k_{F}^{-}}-\lim _{k \rightarrow k_{F}^{+}}\right) n(\mathbf{k}, t)$. A partir de (3.2) podemos obtener una expresión para $Z^{\text {neq }}(t)$,

$$
Z^{\mathrm{neq}}(t)=1-4 \int_{-\infty}^{\infty} \frac{d E}{2 \pi} \frac{\sin ^{2}(E t / 2)}{E^{2}} \Gamma_{\mathbf{k}_{F}}(E)+\mathscr{O}\left(f_{0}^{3}\right),
$$

en donde el scattering rate viene dado por:

$$
\begin{aligned}
\Gamma_{\mathbf{k}_{F}}(E) & =2 \pi \frac{2 f_{0}^{2}}{V^{2}} \sum_{\mathbf{k}, \mathbf{q}}\left|F\left(\mathbf{k}-\mathbf{k}_{F}+\mathbf{q}\right)-F(\mathbf{q})\right|^{2} \delta\left(E+\epsilon(\mathbf{k})-\epsilon\left(\mathbf{k}_{F}+\mathbf{q}\right)-\epsilon(\mathbf{k}-\mathbf{q})\right) \\
& \times\left[n(\mathbf{k}, 0)\left(1-n\left(\mathbf{k}_{F}+\mathbf{q}, 0\right)\right)(1-n(\mathbf{k}-\mathbf{q}, 0))+n\left(\mathbf{k}_{F}+\mathbf{q}, 0\right) n(\mathbf{k}-\mathbf{q}, 0)(1-n(\mathbf{k}, 0))\right],
\end{aligned}
$$

y $\mathbf{k}_{F}$ es un vector sobre la superficie de Fermi (que asumimos circular) y todas las energías se miden con respecto a la energía de Fermi $\epsilon\left(\mathbf{k}_{F}\right)$. Dadas las características de la interacción, el valor asintótico de tiempos largos de la Ec. (3.3) es

$$
Z^{\mathrm{st}}=1-2 \int_{-\infty}^{\infty} \frac{d E}{2 \pi} \frac{\Gamma_{\mathbf{k}_{F}}(E)}{E^{2}}+\mathscr{O}\left(f_{0}^{3}\right) .
$$

Por otro lado, usando técnicas perturvatibas estándar para la autoenergía $\Sigma(\mathbf{k}, E)$ (ver [100] págs. 249-254), es posible demostrar (recordemos que $\Gamma_{\mathbf{k}}(E)=-2 \operatorname{Im} \Sigma(\mathbf{k}, E)$ ) que en equilibrio el residuo de cuasipartícula está dado por

$$
Z^{\mathrm{eq}}=1-\int_{-\infty}^{\infty} \frac{d E}{2 \pi} \frac{\Gamma_{\mathbf{k}_{F}}(E)}{E^{2}}+\mathscr{O}\left(f_{0}^{3}\right),
$$

lo que nos permite relacionar los residuos de cuasipartícula en equilibrio y en el estado estacionario mediante

$$
1-Z^{\mathrm{st}}=2\left(1-Z^{\mathrm{eq}}\right)
$$


También es posible analizar la dinámica $\operatorname{del} Z^{\text {neq }}(t)$. Para tiempos cortos, $Z^{\mathrm{eq}}(t)=1-c t^{2}$, en donde $c \propto f_{0}^{2}$, independientemente de la forma de $F(q)$. Sin embargo, el comportamiento de tiempos más largos, antes de llegar al estado estadionario queda determinado por el comportamiento de $\Gamma_{\mathbf{k}_{F}}(E)$ cuando $E \rightarrow 0$. De hecho, para tiempos largos obtenemos a partir de la Ec. (3.3)

$$
Z^{\mathrm{neq}}(t) \simeq 1+4\left(\int_{1 / t}^{\xi} \frac{d E}{2 \pi} \frac{\Gamma_{\mathbf{k}_{F}}(E)}{E^{2}}-\int_{0}^{\xi} \frac{d E}{2 \pi} \frac{\Gamma_{\mathbf{k}_{F}}(E)}{E^{2}}\right)
$$

en donde $\xi$ es el cutoff en energía impuesto por el decaimiento de $\Gamma_{\mathbf{k}_{F}}(E)$ para energías grandes. De acuerdo con la tería estándar del LF [5, 100], para una superficie de Fermi circular en $D=2$ y una interacción $F(q) \sim e^{-q / q_{c}}, \Gamma_{\mathbf{k}_{F}}(E \rightarrow 0) \sim E^{2+b}$ con $b=0$, lo que implica $Z^{\text {neq }}(t)-Z^{\text {neq }}(\infty) \sim f_{0}^{2} t^{-1}$. Es instructivo, sin embargo, considerar el comportamiento de $Z^{\text {neq }}(t)$ para $b$ arbitrario. Entonces, para $b>-1$ obtendremos un valor finito de $Z^{\text {neq }}(\infty)$ mientras que para $b<-1$ el término dependiente del tiempo en Ec. (3.3) diverge para $t$ grande, es decir, da lugar a lo que se llama un término secular en la expansión perturbativa. La presencia de un término secular en $D=2$ fue descubierta en la Ref. [61] para el modelo de Hubbard en una red cuadrada a llenado medio, el cual posee una superficie de Fermi rectangular. Debido a la presencia de puntos de Van Hove en la superficie de Fermi (en los vértices del rectángulo) el resultado usual de la teoría del LF, $b=0$, no es válido en ese caso, sino que, más bien, estamos ante un caso en el que $b<-1$. Como sea, en nuestro caso $b=0 \mathrm{y}$ $Z^{\text {neq }}(t)=Z^{\text {st }}=$ const. cuando $t \rightarrow+\infty$.

Este comportamiento sugiere la existencia de un plateau en $Z^{\text {neq }}(t)$, el cual ha sido interpretado en trabajos anteriores $[95,96,40]$ como la indicación de un estado estacionario de no equilibrio, un estado pretermalizado. El tratamiento perturbativo provee información muy importante, a saber, que existe un estado estacionario cuyas características pueden relacionarse con las del estado fundamental del Hamiltoniano de evolución y que la relajación viene dada por el dephasing (ver Ec. (3.3)). Sin embargo, surgen las siguientes preguntas: ¿Cuáles son los modos cuasilibres relacionados con la relajación por dephasing? ¿El estado estacionario puede describirse en términos de un GGE? ¿Cómo es la dependencia espaciotemporal de las correlaciones (efecto cono de luz)? Estos interrogantes, los cuales no son accesibles al tratamiento simple con teoría de perturbaciones, pueden ser atacados usando la bosonización, la cual, adicionalmente, nos proveerá de un entendimiento más profundo acerca de la física del problema. Para ello debemos desarrollar la maquinaria de la bosonización multidimensional y aplicarla a nuestro problema fuera de equilibrio, lo cual constituye el propósito de las dos secciones siguientes.

\subsection{Líquidos de Fermi en equilibrio y bosonización}

En esta sección consideraremos brevemente la bosonización de un líquido de Fermi en equilibrio para luego poder construir el tratamiento fuera de equilibrio en la sección siguiente. Para más detalles sobre este tema pueden consultarse las referencias [65, 64, 63, 26, 25, 27, 80]. 
En la presentación subsiguiente seguiremos, en particular, a las Refs. [65] y [80].

Consideremos una excitación tipo partícula-hueco (p-h) por sobre el mar de Fermi. Su energía vendrá dada por:

$$
\epsilon_{\mathbf{k}+\mathbf{q}}-\epsilon_{\mathbf{k}}=\frac{(\mathbf{k}+\mathbf{q})^{2}-\mathbf{k}^{\mathbf{2}^{\prime}}}{2 m} \approx \frac{\mathbf{k} \cdot \mathbf{q}}{m},
$$

asumiendo que $|\mathbf{q}| \ll|\mathbf{k}|$. Esta restricción significa que sólo consideramos excitaciones $\mathrm{p}$-h para las cuales la partícula y el hueco tienen aproximadamente el mismo momento. Esta limitación puede levantarse si notamos que a una dada temperatura, alrededor de la superficie de Fermi habrá tantos huecos como partículas y, sin importar si estos huecos y partículas provienen de excitaciones con momento grande, siempre podemos reagruparlas en pares con momento pequeño. A bajas temperaturas los p-h están cerca de la superficie de Fermi, por lo que $|\mathbf{k}|=k_{F}$. Entonces:

$$
\epsilon_{\mathbf{k}+\mathbf{q}}-\epsilon_{\mathbf{k}}=v_{F} q_{\|},
$$

en donde $q_{\|}$es la componente del momento paralela al vector de Fermi $\mathbf{k}$ sobre la superficie de Fermi, $q_{\|} \equiv \hat{\mathbf{k}} \cdot \mathbf{q}$. Es de particular importancia que la energía del par $\mathrm{p}$-h venga determinada, para excitaciones cercanas a la superficie de Fermi, sólo por el momento, análogamente al caso de una partícula libre. Este argumento es válido en dimensión arbitraria y sentará la base para la descripción bosónica de la física de bajas energías del gas de fermiones.

Consideremos ahora un sistema de fermiones sin espín en $D$ dimensiones interactuando mediante un potencial de a pares $V(\mathbf{x})$. El Hamiltoniano microscópico tendrá entonces la forma:

$$
H=\int d^{D} x c^{\dagger}(\mathbf{x})\left(\frac{-\nabla^{2}}{2 m}\right) c(\mathbf{x})+\frac{1}{2} \int d^{D} x d^{D} y V(\mathbf{x}-\mathbf{y}) c^{\dagger}(\mathbf{x}) c^{\dagger}(\mathbf{y}) c(\mathbf{x}) c(\mathbf{y}),
$$

en donde $c^{\dagger}(\mathbf{x}), c(\mathbf{x})$ son los operadores de creación y aniquilación canónicos de los fermiones desnudos. Dado que bajas temperaturas las excitaciones relevantes viven cerca de la superficie de Fermi es conveniente trabajar con el Hamiltoniano efectivo que gobierna la dinámica de esas excitaciones. Formalmente debemos integrar los modos más energéticos mediante un procedimiento análogo al grupo de renormalización. Como resultado obtendremos una acción (o Hamiltoniano) efectiva que describa la física de las cuasipartículas resultantes. Antes de explicitar este Hamiltoniano efectivo definiremos otras cantidades importantes.

Las cuasipartículas no son necesariamente cuasipartículas de Landau, sino que están relacionadas con los fermiones desnudos a través de [132]

$$
\psi_{\mathbf{k}}=Z_{\mathbf{k}}^{-1 / 2} c_{\mathbf{k}}
$$

para momentos $\mathbf{k}$ restringidos a una cáscara fina de grosor $\lambda$ alrededor de la superficie de 

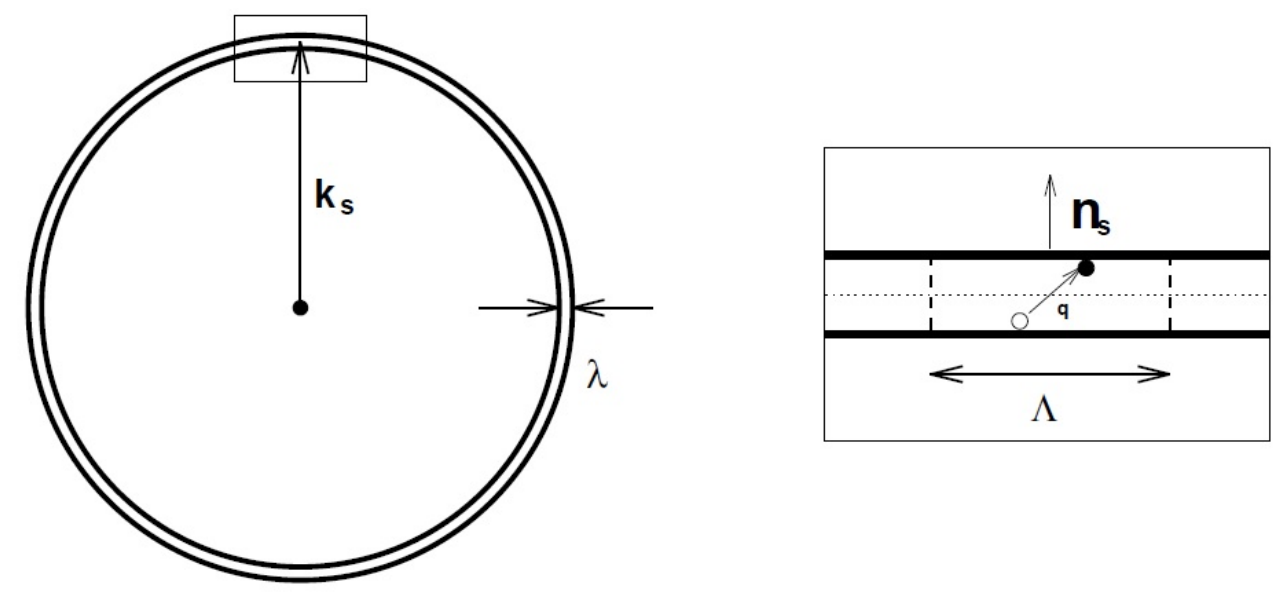

Figura 3.1: La capa delgada de ancho $\lambda$ contiene a la superficie de Fermi. La capa está dividida en cajas de ancho $\Lambda$, etiquetadas con un rótulo $\mathbf{S}$. El zoom muestra una de las cajas que contiene un par p-h de momento q. Es necesario que $|\mathbf{q}|<\lambda \ll \Lambda \ll k_{F}$ para poder implementar la bosonización en equilibrio. Tomado de [65].

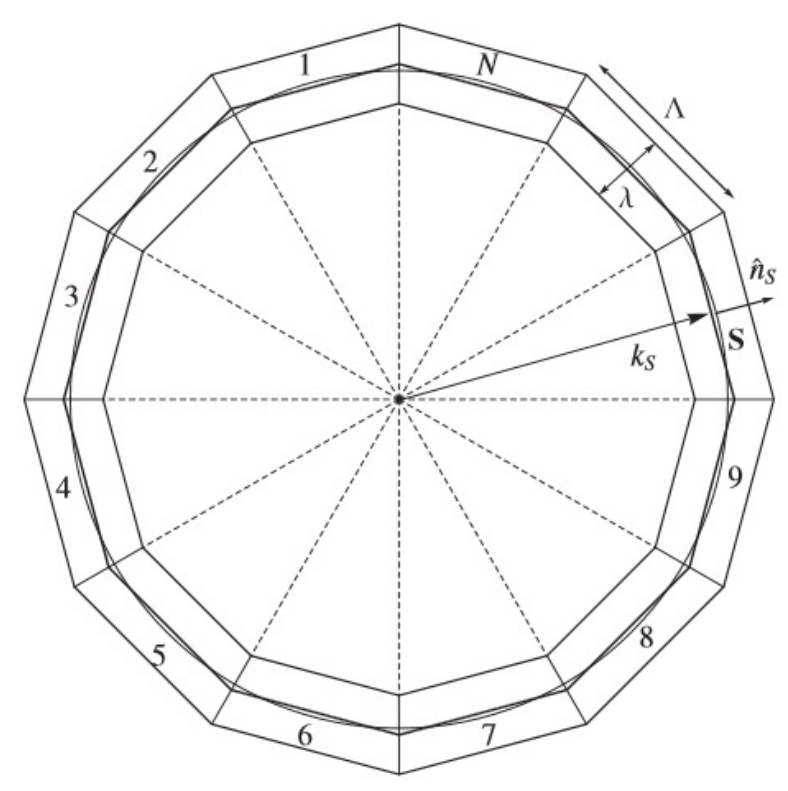

Figura 3.2: Superficie de Fermi isotrópica en $D=2$ dividida en $N$ parches de dimensiones $\lambda$ y $\Lambda$ en las direcciones perpendicular y paralela a la superficie de Fermi, respectivamente. La magnitud del cutoff $\Lambda$ es elegida de manera que el vector normal a la superficie de Fermi no varíe apreciablemente dentro de un dado parche y pueda ser reemplazado por un vector constante $\hat{\mathbf{n}}_{\mathbf{S}}$, lo que permite linealizar la relación de dispersión en cada parche (ver más adelante en el texto). Tomado de [39]. 
Fermi $k_{F}-\lambda / 2<|\mathbf{k}|<k_{F}+\lambda / 2^{1}$. Para implementar la bosonización, dividimos la superficie de Fermi en $N$ cajas o parches de alto $\lambda$ perpendicular a la superficie de Fermi y dimensiones $\Lambda^{D-1}$ sobre la superficie, como puede apreciarse en las Figuras 3.1 y 3.2.Más adelante veremos que uno de los requisitos para que el tratamiento con bosonización funcione es que los cutoffs deben ser tales que $k_{F} \gg \Lambda \gg \lambda$ lo que determina una relación de aspecto achatada para los compartimentos de la superficie de Fermi. Además, introducimos los operadores $\psi_{\mathbf{S}}(\mathbf{x})$ y $\psi_{\mathbf{S}}^{\dagger}(\mathbf{x})$ que aniquilan y crean cuasipartículas fermiónicas dentro de cada parche en la posición $\mathbf{x}$ :

$$
\psi_{\mathbf{S}}(\mathbf{x})=e^{i \mathbf{k}_{\mathbf{s}} \cdot \mathbf{x}} \sum_{p} \theta(\mathbf{S} ; \mathbf{p}) e^{i\left(\mathbf{p}-\mathbf{k}_{\mathbf{s}}\right) \cdot \mathbf{x}} \psi_{\mathbf{p}}
$$

en donde $\theta(\mathbf{S} ; \mathbf{p})$ es 1 si $\mathbf{p}$ está dentro del parche $\mathbf{S}$ y 0 si no. En $D=1$ la superficie de Fermi se reduce a dos puntos y por lo tanto debemos utilizar sólo dos cajas (caja $L$ y caja $R$ ). En una dimensión las interacciones pueden clasificarse en aquellas que dejan a los fermiones en la misma caja (interacciones $g_{4}$ en el capítulo anterior) e interacciones que intercambian de caja a los fermiones (interacciones $g_{2}$ ). En $D>1$ es posible hacer una clasificación análoga. Nos limitaremos al caso particular de $D=2$ por simplicidad. Primero notemos que en función de los operadores $\psi_{\mathbf{S}}(\mathbf{x})$ la interacción efectiva entre las cuasipartículas es una interacción de a pares:

$$
\psi_{\mathbf{S}}^{\dagger}(\mathbf{x}) \psi_{\mathbf{T}}^{\dagger}(\mathbf{y}) \psi_{\mathbf{U}}(\mathbf{y}) \psi_{\mathbf{V}}(\mathbf{x})
$$

Esta es la única interacción marginal dado que todos los términos con más operadores o derivadas son irrelevantes en el lenguaje del grupo de renormalización [132]. El Hamiltoniano desnudo, Ec. (3.11) es invariante rotacional y por lo tanto podemos restringir nuestra atención a interacciones efectivas que también sean invariantes frente a rotaciones. La interacción está fuertemente constreñida por la conservación del momento, es decir, por el hecho de que todas las interacciones entre partículas deben suceder dentro de la cáscara de grosor $\lambda$ alrededor de la superficie de Fermi:

$$
\left|k_{\mathbf{U}}+k_{\mathrm{V}}-k_{\mathrm{S}}-k_{\mathrm{T}}\right|<\lambda .
$$

En $D=2$ hay sólo tres posibilidades: $\mathbf{V}=\mathbf{S}$ y $\mathbf{U}=\mathbf{T}$ (forward scattering, análogo al $g_{4} \mathrm{de}$ una dimensión), $\mathbf{U}=\mathbf{S}$ y $\mathbf{V}=\mathbf{T}$ (exchange scattering, interacción de intercambio), y $\mathbf{S}=-\mathbf{T}$ y $\mathbf{U}=-\mathbf{V}$ (BCS scattering, acoplamiento de pares de Cooper, en donde el signo - denota el punto antipodal). Las interacciones en los canales de forward y exchange scattering poseen un grupo de simetría extendido $U(1)_{k}^{N}$ : una rotación U(1) en la fase de los operadores correspondientes a cada parche deja invariantes a las interacciones en estos dos canales dado que los parches $\mathbf{S}$ y T contienen un oprador de creación y uno de aniquilación cada uno. Esta simetría, la cual no está presente en el Hamiltoniano microscópico, es la que permitirá resolver exactamente

\footnotetext{
${ }^{1}$ Las cuasipartículas de Landau están definidas en un entorno infinitesimal de la superficie de Fermi y su relación con los fermiones desnudos es por lo tanto $\psi_{\mathbf{k}}^{L}=Z_{F}^{-1 / 2} c_{\mathbf{k}}$, en donde $Z_{F}$ es el residuo de cuasipartícula, la discontinuidad en el momento de Fermi de la distribución de momentos de los fermiones desnudos.
} 
al modelo efectivo de las cuasipartículas. Notemos también que la teoría de Landau posee una especie de simetría $U(1)_{k}^{\infty}$, dado que el número de cuasipartículas en cualquier punto de la superficie de Fermi está conservado. La interacción BCS, la cual acopla cuasipartículas en cuatro parches diferentes, rompe la simetría $U(1)_{k}^{N}$ y deja sólo la simetría global $U(1)$ que simplemente refleja la conservación de la carga, la simetría original del modelo microscópico. No consideraremos las interacciones BCS las cuales, como es bien sabido, representan una de las inestabilidades del LF.

El Hamiltoniano efectivo para las cuasipartículas en términos de los campos en cada parche puede escribirse como:

$$
H=\int d^{D} x \sum_{\mathbf{S}} \psi_{\mathbf{S}}^{\dagger}(\mathbf{x})\left(\frac{\mathbf{k}_{\mathbf{S}}}{m^{*}} \cdot \frac{\nabla}{i}\right) \psi_{S}(\mathbf{x})+\frac{1}{2} \int d^{D} x d^{D} y \sum_{\mathbf{S}, \mathbf{T}} f(\mathbf{S}, \mathbf{T} ; \mathbf{x}-\mathbf{y}) \psi_{\mathbf{S}}^{\dagger}(\mathbf{x}) \psi_{\mathbf{S}}(\mathbf{x}) \psi_{\mathbf{T}}^{\dagger}(\mathbf{y}) \psi_{\mathbf{T}}(\mathbf{y})
$$

En donde $m^{*}$ es la masa efectiva de las cuasipartículas y $f(\mathbf{S}, \mathbf{T} ; \mathbf{x}-\mathbf{y})$ es la interacción efectiva entre las cuasipartículas, es decir, es la interacción «vestida» que surge al integrar los grados de libertad de alta energía. Además hemos linealizado la relación de dispersión discutida al principio: $\frac{\mathbf{k s} \cdot \mathbf{q}}{m^{*}}$ pasa a ser en el espacio de corrdenadas $\frac{\mathbf{k} \mathbf{s}}{m^{*}} \cdot \frac{\nabla}{i}$.

De manera completamente análoga al caso unidimensional, para llevar a cabo la bosonización introducimos los operadores de corriente en cada parche:

$$
J_{\mathbf{S}}(\mathbf{q})=\sum_{k} \theta(\mathbf{S} ; \mathbf{k}-\mathbf{q}) \theta(\mathbf{S} ; \mathbf{k})\left(\psi_{\mathbf{k}-\mathbf{q}}^{\dagger} \psi_{\mathbf{k}}-\delta_{\mathbf{q}, \mathbf{0}} n_{\mathbf{k}}\right)
$$

en donde $n_{\mathbf{k}}=\left\langle\psi_{\mathbf{k}}^{\dagger} \psi_{\mathbf{k}}\right\rangle$ asegura el orden normal de la expresión. La corriente $J_{\mathbf{S}}(-\mathbf{q})$ crea un par p-h de momento relativo $\mathbf{q}$ en el parche $\mathbf{S}$ de la superficie de Fermi. Estos son los objetos clave para la bosonización. En efecto, puede mostrarse que obedecen (aproximadamente) el álgebra $U(1)$

$$
\left[J_{\mathbf{S}}(\mathbf{q}), J_{\mathbf{T}}(\mathbf{p})\right]=\delta_{\mathbf{S}, \mathbf{T}} \delta_{\mathbf{q}+\mathbf{p}} \Omega \hat{\mathbf{n}}_{\mathbf{S}} \cdot \mathbf{q}+\text { Error, }
$$

en donde $\Omega \equiv \Lambda^{D-1}\left(\frac{L}{2 \pi}\right)^{D}$ es el número de estados en el parche dividido por el cutoff $\lambda \mathrm{y}$ $\hat{\mathbf{n}}_{\mathbf{S}} \equiv \frac{\mathbf{k}_{\mathbf{s}}}{\left|\mathbf{k}_{\mathbf{s}}\right|}$. El Error en la ecuación (3.18) es despreciable comparado con el término principal siempre que elijamos $\lambda / \Lambda \ll 1$. Esto está señalando que la relación de aspecto de los parches es fundamental para la construcción. Físicamente, elegir parches achatados minimiza el scattering de partículas entre parches adyacentes. Además, dado que (para una superficie de Fermi circular) el vector normal a la superficie de Fermi varía en una escala del orden de $k_{F}$, para poder implementar la linealización de la relación de dispersión dentro de cada parche debemos pedir que $\Lambda \ll k_{F}$. Otro punto importante que surge al calcular el término de error en la Ec. (3.18) es que la ocupación de cuasipartícula debe cambiar de 1 a 0 en un rango de momentos del orden de $\lambda$, es decir, para que la bosonización funcione debemos considerar estados a muy baja temperatura. 
Una vez establecida las relaciones de conmutación podemos generar la representación bosónica. Para ello definimos los operadores bosónicos $a_{\mathbf{S}}(\mathbf{q})$ de modo que:

$$
J_{\mathbf{S}}(\mathbf{q})=\sqrt{\Omega\left|\hat{\mathbf{n}}_{\mathbf{S}} \cdot \mathbf{q}\right|}\left[a_{\mathbf{S}}(\mathbf{q}) \theta\left(\hat{\mathbf{n}}_{\mathbf{S}} \cdot \mathbf{q}\right)+a_{\mathbf{S}}^{\dagger}(-\mathbf{q}) \theta\left(-\hat{\mathbf{n}}_{\mathbf{S}} \cdot \mathbf{q}\right)\right] .
$$

Es decir, si elegimos a los $a_{\mathbf{s}}(\mathbf{q})$ como operadores bosónicos canónicos

$$
\left[a_{\mathbf{S}}(\mathbf{q}), a_{\mathbf{T}}^{\dagger}(\mathbf{p})\right]=\delta_{\mathbf{S}, \mathbf{T}} \delta_{\mathbf{q}, \mathbf{p}}
$$

y escribirmos la corriente en función de ellos tal como figura en la Ec. (3.19) la relación de conmutación (3.18) se satisfacen automáticamente con el término de error igual a cero.

Definiendo el campo bosónico

$$
\phi_{\mathbf{S}}(\mathbf{x})=i \sum_{\mathbf{q}, \mathbf{q} \cdot \hat{\mathbf{n}}_{\mathbf{S}}>0} \frac{a_{\mathbf{S}}^{\dagger}(-\mathbf{q}) e^{-i \mathbf{q} \cdot \mathbf{x}}-a_{\mathbf{S}}(\mathbf{q}) e^{i \mathbf{q} \cdot \mathbf{x}}}{\sqrt{\hat{\mathbf{n}}_{\mathbf{S}} \cdot \mathbf{q}}}
$$

podemos escribir inmediatamente a la transformada de Fourier de la corriente en el parche $J_{\mathbf{S}}(\mathbf{x})=\sum_{\mathbf{q}} e^{i \mathbf{q} \cdot \mathbf{x}} J_{\mathbf{S}}(\mathbf{q})$ como

$$
J_{\mathbf{S}}(\mathbf{x})=\sqrt{\Omega} \hat{\mathbf{n}}_{\mathbf{S}} \cdot \nabla \phi_{\mathbf{S}}(\mathbf{x}) .
$$

También es posible escribir el Hamiltoniano con la relación de dispersión linealizada Ec. (3.16) en términos de los operadores de corriente $J_{\mathbf{S}}(\mathbf{x})$ y, por lo tanto, de los operadores bosóni$\cos [65]$ :

$$
H=\int d^{2} x \sum_{\mathbf{S}} \frac{\pi v_{F}^{*}}{\Omega}\left[\hat{\mathbf{n}}_{\mathbf{S}} \cdot \nabla \phi_{\mathbf{S}}(\mathbf{x})\right]^{2}+\frac{1}{V^{2}} \int d^{2} x d^{2} y \sum_{\mathbf{S}, \mathbf{T}} f(\mathbf{S}, \mathbf{T} ; \mathbf{x}-\mathbf{y})\left[\hat{\mathbf{n}}_{\mathbf{S}} \cdot \nabla \phi_{\mathbf{S}}(\mathbf{x})\right]\left[\hat{\mathbf{n}}_{\mathbf{T}} \cdot \nabla \phi_{\mathbf{T}}(\mathbf{y})\right],
$$

en donde $v_{F}^{*}=k_{F} / m^{*}$ es la velocidad de Fermi renormalizada de las cuasipartículas. El Hamiltoniano (3.23) puede escribirse fácilmente en términos de los operadores $a_{\mathbf{S}}(\mathbf{q})$ usando la Ec. (3.21) y luego puede diagonalizarse utilizando una especie de extensión multidimensional de la transformación de Bogoliubov utilizada en el capítulo anterior para el caso unidimensional. Este punto será analizado en detalle cuando ataquemos el problema fuera de equilibrio.

Puede mostrarse que los estados coherentes bosónicos que son autoestados de los operadores de aniquilación $a_{\mathbf{S}}(\mathbf{q})$ generan, al aplicarse sobre el mar de Fermi un desplazamiento en la superficie de Fermi en la dirección del momento q [26]. Por lo tanto, se relaciona a los modos bosónicos con las fluctuaciones en la forma de la superficie de Fermi generados por una superposición coherente de excitaciones de p-h. 
Para el cálculo de las funciones de correlación necesitamos una identidad de bosonización multidimensional análoga a la que existe en una dimensión (Ec. (2.16)). Aunque para $D>1$ tal identidad aún no ha podido ser deducida usando el enfoque de operadores que utilizamos aquí (aunque se han proporcionado argumentos de plausibilidad en su favor), otros enfoques que utilizan integrales funcionales tales como [ 80] obtienen los mismos resultados que si se usara el enfoque con operadores y una identidad de bosonización dada por:

$$
\psi_{\mathbf{S}}(\mathbf{x})=\sqrt{\frac{\Omega}{V a}} e^{i \mathbf{k}_{\mathbf{s}} \cdot \mathbf{x}} \exp \left\{\frac{i}{\sqrt{\Omega}} \phi_{\mathbf{S}}(\mathbf{x})\right\},
$$

en donde $V$ es el volumen del sistema y $a \equiv 1 / \lambda$ es el cutoff ultravioleta.

Resulta muy interesante que toda esta maquinaria reproduce, en equilibrio, los resultados de la teoría de Landau (la cual fue desarollada diagramáticamente por Abrikosov y Khalatnikov más adelante) incluyendo la presencia de modos colectivos (el sonido cero, por ejemplo), los cuales están directamente relacionados con los operadores bosónicos $a_{\mathbf{S}}(\mathbf{q})$ [25]. La maquinaria de bosonización también reproduce el comportamiento de largas distancias de los propagadores fermiónicos en un LF [64]. Adicionalmente, se cree que la bosonización en altas dimensiones, debido a su carácter no perturbativo, puede ser una herramienta poderosa para tratar problemas que no pueden ser alcanzados con teoría de perturbaciones. Un ejemplo de esto es el problema de un sistema de fermiones con interacciones singulares (de largo alcance o retardadas), un modelo que presenta comportamientos que no son LF y que puede servir para describir diferentes situaciones tales como transiciones magnéticas en los cupratos, efecto Hall cuántico y la transición de Pomeranchuk (sistemas que poseen sólo interacciones de corto alcance en el modelo microscópico). Sin embargo, la validez de los resultados de la bosonización en estos casos más desafiantes es objeto de debates [34].

\subsection{Quenches en la interacción y bosonización}

En esta sección describiremos cómo hay que modificar el enfoque de la bosonización en equilibrio, descripto antes, para poder calcular funciones de correlación después de un quench de interacción en un gas de fermiones en $D>1$. Como iremos viendo, este método permite rederivar los resultados obtenidos con el método perturbativo. Lo que es más importante, también permite obtener el modelo integrable efectivo que describe la dinámica en el régimen de pretermalización y las cantidades conservadas asociadas.

Ya hemos señalado en la sección anterior que consideramos una interacción débil ( $f_{0}$ pequeño comparado con la energía de Fermi) y de largo alcance $\left(F(q)\right.$ decae rápidamente para $q>q_{c}$ ). En lo que sigue mostraremos por qué estos requisitos, que no deben asumirse al bosonizar en equilibrio, son cruciales para poder hacer la bosonización después del quench. Para ello, utilizaremos ciertos argumentos basados en el cálculo perturbativo presentado antes. Basaremos nuestro argumento en la Ec. (3.2), válida para tiempos cortos, la cual repetimos 
aquí para facilitar la lectura

$$
\begin{aligned}
n(\mathbf{k}, t) & =n(\mathbf{k}, 0)-\frac{8 f_{0}^{2}}{V^{2}} \sum_{\mathbf{k}^{\prime}, \mathbf{q}}\left|F\left(\mathbf{k}^{\prime}-\mathbf{k}+\mathbf{q}\right)-F(\mathbf{q})\right|^{2} \frac{\sin ^{2}\left[\frac{t}{2}\left(\epsilon(\mathbf{k})+\epsilon\left(\mathbf{k}^{\prime}\right)-\epsilon(\mathbf{k}+\mathbf{q})-\epsilon\left(\mathbf{k}^{\prime}-\mathbf{q}\right)\right)\right]}{\left(\epsilon(\mathbf{k})+\epsilon\left(\mathbf{k}^{\prime}\right)-\epsilon(\mathbf{k}+\mathbf{q})-\epsilon\left(\mathbf{k}^{\prime}-\mathbf{q}\right)\right)^{2}} \\
& \times\left[n(\mathbf{k}, 0) n\left(\mathbf{k}^{\prime}, 0\right)(1-n(\mathbf{k}+\mathbf{q}, 0))\left(1-n\left(\mathbf{k}^{\prime}-\mathbf{q}, 0\right)\right)-\right. \\
& \left.i n(\mathbf{k}+\mathbf{q}, 0) n\left(\mathbf{k}^{\prime}-\mathbf{q}, 0\right)(1-n(\mathbf{k}, 0))\left(1-n\left(\mathbf{k}^{\prime}, 0\right)\right)\right]+\mathscr{O}\left(f_{0}^{3}\right) .
\end{aligned}
$$

Esta ecuación nos muestra que a orden $f_{0}^{2}$, la dinámica de tiempos cortos está completamente determinada por el espacio de fases inicial para las colisiones entre los fermiones desnudos y también por las propiedades del potencial. Consideremos ahora, igual que en equilibrio, una capa de grosor $\lambda$ alrededor de la superficie de Fermi. Es relativamente fácil convencerse de que, dada la forma particular de la condición inicial, todos los términos no nulos en (3.2) que mezclan estados dentro y fuera de esa capa van multiplicados por un factor del orden de $f_{0}^{2}|F(\lambda / 2)|^{2}$. Eligiendo $\lambda / 2>q_{c}$ este factor está fuertemente suprimido dada la forma del potencial de interacción. En otras palabras, el ritmo de ocurrencia de tales eventos de scattering es aproximadamente $\sim f_{0}^{2}|F(\lambda)|^{2} N(0) \sim \tau^{-1}$, en donde $N(0)=\frac{k_{F}}{2 \pi v_{F}}$ es la densidad de estados a la energía de Fermi. Si $\lambda>q_{c}$ tales procesos están fuertemente suprimidos, y por lo tanto su influencia sobre la dinámica es despreciable para $t \lesssim \tau$. Consecuentemente, podemos restringir las sumas en $\mathbf{k}^{\prime}$ y $\mathbf{q}$ a valores que estén dentro de la capa de grosor $\lambda$ y confiar en los resultados a orden $f_{0}^{2}|F(\lambda / 2)|^{2}$. Notemos entonces que las sumas truncadas son equivalentes a la dinámica completa generada por un Hamiltoniano truncado, que llamaremos $H^{F S}$, el cual se obtiene de $H$ conservando en las sumas sobre momentos sólo los términos que están dentro de la capa. Un análisis similar puede hacerse para otros observables que no sean $n(\mathbf{k}, t)$. Concluimos entonces que para tiempos $t \lesssim 1 / f_{0}^{2}|F(\lambda / 2)|^{2} N(0)$, la dinámica de los grados de libertad dentro de la capa están descriptos efectivamente por $H^{F S}$.

Recapitulando, vemos que las caracerísticas de la interacción en combinación con las de la condición inicial nos aseguran que la dinámica se desarrollará, aproximadamente, a una distancia pequeña de la superficie de Fermi, lo que abre la puerta a un tratamiento con bosonización. En equilibrio, lo que nos permitía quedarnos cerca de la superficie de Fermi era el tratamiento con el Grupo de Normalización. Si considerarámos interacciones de corto alcance (si $q_{c}$ fuera una fracción apreciable de $k_{F}$ por ejemplo) los procesos de scattering con alta transferencia de momentos serían tan probables como aquellos con baja transferencia de momentos y la dinámica se alejaría rápidamente de la supeficie de Fermi. Por otro lado, si consideráramos una condición inicial en la que los grados de libertad de alta energía ya estuvieran poblados (como en el caso de un estado de alta temperatura) la dinámica tampoco estaría restringida a las vecindades de la superficie de Fermi.

Para describir la dinámica generada por $H^{F S}$ implementaremos el tratamiento con bosonización. Primero recordemos la definición de las funciones

$$
\Theta(\mathbf{S} ; \mathbf{p})= \begin{cases}1 & \text { si } \mathbf{p} \in \text { parche } \mathbf{S} \\ 0 & \text { en otro caso. }\end{cases}
$$


Ahora también introduciremos las funciones ausxiliares $\tilde{\Theta}(\mathbf{S} ; \mathbf{p})$ que son cero si $\mathbf{p}$ está en el parche $\mathbf{S}$ y no cero en una región complementaria de ese parche de manera que

$$
\sum_{\mathbf{S}} \Theta(\mathbf{S} ; \mathbf{p})+\tilde{\Theta}(\mathbf{S} ; \mathbf{p})=1
$$

lo que significa que la superposición de los parches y sus regiones complementarias correspondientes cubren todo el espacio de momentos. Para aislar los grados de libertad que están cerca de la superficie de Fermi, introducimos la relaciónd de completitud Ec. (3.27) repetidamente en la parte de interacción del Hamiltoniano fermiónico y agrupamos los términos de la siguiente manera

$$
H_{\mathrm{int}}=\frac{1}{V} \sum_{\mathbf{S}, \mathbf{T}, \mathbf{S}^{\prime}, \mathbf{T}^{\prime}, \mathbf{q} \mathbf{k}_{1}, \mathbf{k}_{2}} f(q) \Theta\left(\mathbf{S} ; \mathbf{k}_{1}\right) \Theta\left(\mathbf{S}^{\prime} ; \mathbf{k}_{1}+\mathbf{q}\right) \Theta\left(\mathbf{T} ; \mathbf{k}_{2}\right) \Theta\left(\mathbf{T}^{\prime} ; \mathbf{k}_{2}-\mathbf{q}\right) Q_{\mathbf{k}_{1}}(\mathbf{q}) Q_{\mathbf{k}_{2}}(-\mathbf{q})+\tilde{H}_{\mathrm{int}},
$$

Todos los términos en $\tilde{H}_{\text {int }}$ contienen al menos una $\tilde{\Theta}$, es decir, es el Hamiltoniano que involucra a los grados de libertad de alta energía. Análogamente al caso de equilibrio, un examen cuidadoso de Eq. (3.28) revela que existen sólo tres tipos de términos no nulos en la suma [63], $\mathbf{S}=\mathbf{S}^{\prime}$ y $\mathbf{T}=\mathbf{T}^{\prime}$ (forward scattering), $\mathbf{S}=\mathbf{T}^{\prime}$ y $\mathbf{T}=\mathbf{S}^{\prime}$ (intercambio) or $\mathbf{T}=-\mathbf{S}$ and $\mathbf{T}^{\prime}=-\mathbf{S}^{\prime}$ (interacción de pares de Cooper). Introduciendo las densidades en el parche

$$
J_{\mathbf{S}}(\mathbf{q})=\sum_{\mathbf{k}} \Theta(\mathbf{S} ; \mathbf{k}+\mathbf{q}) \Theta(\mathbf{S} ; \mathbf{k}) Q_{\mathbf{k}}(\mathbf{q}),
$$

la parte de forward scattering puede escribirse como

$$
H_{\mathrm{int}}^{F S}=\frac{1}{V} \sum_{\mathbf{S}, \mathbf{T}, \mathbf{q}} f(q) J_{\mathbf{S}}(\mathbf{q}) J_{\mathbf{T}}(-\mathbf{q}),
$$

Los términos BCS están relacionados con una alta transferencia de momento ( $q$ es una fracción apreciable de $k_{F}$ ) y son inocuos a tiempos cortos. Los términos de intercambio no pueden ser escritos en términos de las densidades en el parche. Sin embargo, los únicos términos de intercambio de los que debemos preocuparnos son aquellos que transfieren fermiones entre parches adyacentes. Los otros están relacionados con una transferencia de momentos $q>\lambda / 2$ y podemos despreciarlos en nuestro nivel de aproximación. Los procesos de intercambio entre parches adyacentes asociados con una transferencia de momentos $q<\lambda / 2$ (y por lo tanto relevantes a tiempos cortos) son aquellos que tienen lugar dentro de la región sombreada en la Fig. 3.3. El área de la región sombreada es $\lambda^{2}$, lo que significa que los procesos posibles dentro de esta área son del orden de $\lambda^{4}$ en el límite $V \rightarrow \infty$. Por otro lado, el número de procesos que ocurren dentro de los parches (y que están incluídos en los términos de forwrd scattering) es del orden de $\lambda^{2} \Lambda^{2}$. Entonces la razón entre ellos es del orden de $\Lambda^{2} / \lambda^{2} \gg 1$. Sobre esta base podemos despreciar los procesos de interacambio que tienen lugar dentro de la región sombreada. Esta es la razón por la que pueden despreciarse también en equilibrio [80]. La adaptación de estos argumentos a $D=1$ permite despreciar los procesos de backscattering ( $g_{1}$ en la notación de la $g$-ología) a tiempos cortos. Los procesos de 
forward scattering relevantes son las interacciones $g_{4}$ (diagonales en el parche) y $g_{2}$ (mezcla las ramas derecha e izquierda del espectro). Esto representa una justificación al hecho de que las predicciones del ML describen la dinámica de tiempos cortos de un sistema en la red, siempre que la condición inicial no tenga poblados estados de alta energía y que el quench sea débil.

Recordamos ahora que las corrientes en el parche $J_{\mathbf{S}}(\mathbf{q})$ obedecen las relaciones de conmutación anómalas, Ec. (3.18), y que si $\Lambda \gg \lambda$ tales operadores pueden ser representados con operadores bosónicos canónicos $\left\{a_{\mathbf{S}}(\mathbf{q}), a_{\mathbf{S}}^{\dagger}(\mathbf{q})\right\}$ :

$$
J_{\mathbf{S}}(\mathbf{q})=\sqrt{\Omega\left|\hat{\mathbf{n}}_{\mathbf{S}} \cdot \mathbf{q}\right|}\left[a_{\mathbf{S}}^{\dagger}(\mathbf{q}) \theta\left(\hat{\mathbf{n}}_{\mathbf{S}} \cdot \mathbf{q}\right)+a_{\mathbf{S}}(-\mathbf{q}) \theta\left(-\hat{\mathbf{n}}_{\mathbf{S}} \cdot \mathbf{q}\right)\right],
$$

en donde $\theta(x)$ es la fnción de Heaviside. Entonces, la porción de forward scattering del Hamiltoniano de interacción Ec. (3.30) ya está bosonizada. Para bosonizar la parte cinética hay que hacer más aproximaciones. Como con el Hamiltoniano de interacción, primero separamos el término cinético del Hamiltoniano en partes con momento alto y bajo, lo cual puede hacerse formalmente utilizando las relaciones de clausura de las funciones $\Theta(\mathbf{S} ; \mathbf{p})$. Obtenemos

$$
H_{0}=\sum_{\mathbf{S}, \mathbf{q}} \epsilon\left(\mathbf{k}_{\mathbf{s}}+\mathbf{q}\right) c_{\mathbf{k}_{\mathbf{s}}+\mathbf{q}}^{\dagger} c_{\mathbf{k}_{\mathbf{s}}+\mathbf{q}}+\tilde{H}_{0}
$$

en donde $\mathbf{q}$ debe ser lo suficientemente pequeño como para entrar dentro del parche $\mathbf{S}$. En virtud de los argumentos presentados antes, despreciamos la parte con momento alto $\tilde{H}_{0}$. Además, asumimos que el tamaño del parche es pequeño comparado con la escala en la que la superficie de Fermi cambia de forma. Como fue dicho antes, para una superficie de Fermi circular es suficiente pedir que $\Lambda \ll k_{F}$. En este caso podemos despreciar las variaciones del vector normal a la superficie de Fermi dentro del parche, lo que hace posible linealizar la relación de dispersión dentro de cada parche [80]. Adicionalmente, si sólo nos concentramos en los grados de libertad de baja energía, en virtud de las relaciones de conmutación (3.18), podemos escribir la parte cinética del Hamiltoniano como $[63,26]$

$$
H_{0}^{F S}=\frac{v_{F}}{2 \Omega} \sum_{\mathbf{S}, \mathbf{q}} J_{\mathbf{S}}(\mathbf{q}) J_{\mathbf{S}}(-\mathbf{q})
$$

en donde $v_{F}=\left|\nabla_{\mathbf{k}} \epsilon(\mathbf{k})\right|_{|\mathbf{k}|=k_{F}}$ es la velocidad de Fermi de los fermiones desnudos. El Hamiltoniano en término de las corrientes puede escribirse finalmente como

$$
H^{F S}=\sum_{\mathbf{S}, \mathbf{T}, \mathbf{q}} J_{\mathbf{S}}(\mathbf{q})\left(\frac{v_{F}}{2 \Omega} \delta_{\mathbf{S}, \mathbf{T}}+\frac{f(q)}{V}\right) J_{\mathbf{T}}(-\mathbf{q}) .
$$

Nótese que la colección de condiciones sobre los cutoffs es consistente:

$$
k_{F} \gg \Lambda \gg \lambda>q_{c} .
$$


Esperamos entonces que las aproximaciones descriptas sean precisas para altas densidades ( $k_{F}$ grande) y para tiempos menores que $\tau$.

El Hamiltoniano $H^{F S}$ cumple el mismo papel que el Hamiltoniano de cuasipartícula (3.16) cumplía en equilibrio. La gran diferencia es que en el Hamiltoniano de cuasipartícula aparecían cantidades renormalizadas (masas e interacciones) mientras que en $H^{F S}$ aparecen cantidades desnudas. Esta es la gran diferencia entre los tratamientos en equilibrio y fuera de equilibrio. Por lo tanto, $H^{F S}$ no describe la física de bajas energías del Hamiltoniano microscópico $H$ sino que describe una etapa temprana en la dinámica luego del encendido abrupto de las interacciones en la que los fermiones aún no se han «vestido» por efecto de las interacciones, es decir, la etapa de formación de las cuasipartículas. Sobre este punto hacemos notar que en $D=1$ ha sido encontrado recientemente que la dinámica luego de un quench de interacción puede ser descripta por la teoría bosónica de bajas energías parametrizada con los parámetros desnudos del Hamiltoniano microscópico en vez de con los parámetros renormalizados de equilibrio [60]. Además, $H^{F S}$ es integrable, ya que puede expresarse como un bilineal de opradores bosónicos lo cual, al igual que en equilirbio, está íntimamente ralacionado con la simetría $U^{N}(1)_{k}$ del Hamiltoniano $H^{F S}$. Según lo que vimos en el capítulo anterior, esto significa que la relajación descripta por $H^{F S}$ desembocará en un estado estacionario descripto por un GGE, un estado altamente no térmico. Correcciones de orden mayor (anarmónicas) a (3.34) tales como las que surgen de la curvatura de la superficie de Fermi y la relación de dispersión, introducen interacciones entre los bosones [80]. Estos términos violan el conjunto de leyes de conservación que hacen exactamente soluble al Hamiltoniano. Como consecuencia, se espera que a tiempos largos ocurra una relajación completa al equilibrio térmico. Este fenómeno está controlado sólo por las leyes de conservación que son respetadas por el Hamiltoniano real (una simetría $U(1)$ global), el cual difiere del Hamiltoniano truncado integrable en que también describe colisiones que no son forward scattering.

En la teoría de Landau se suponía un encendido adiabático de las interacciones en el cual los fermiones tenían todo el tiempo del mundo para vestirse de acuerdo al valor instantáneo de las interacciones. En el caso del quench existe una primera etapa de la dinámica en la cual los fermiones interactúan, pero las cuasipartículas aún no están formadas (y por ello no colisionan), esta es la etapa de pretermalización y de la dinámica por dephasing.

\subsection{Cálculo de las funciones de correlación después de un quench utilizando bosonización}

Ya tenemos los resultados del análisis perturbativo del quench y contamos con una maquinaria de bosonización fuera de equilibrio para atacar el problema desde una óptica completamente diferente. Falta ahora calcular las funciones de correlación luego del quench utilizando el formalismo de bosonización y comparar los resultados. Como puede preverse esto no es una tarea fácil y plantea un desafío técnico importante. En esta sección explicaremos cómo puede resolverse este problema. 


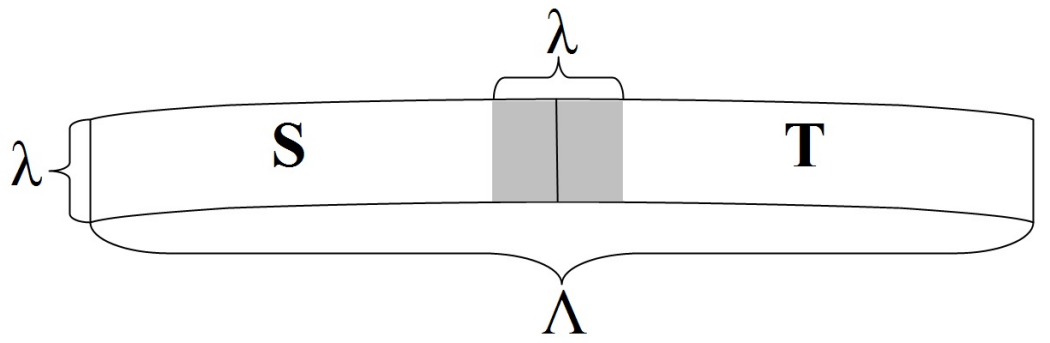

Figura 3.3: Los procesos de intercambio entre dos parches adyacentes $\mathbf{S}$ y $\mathbf{T}$ con transferencia de momento $q<\lambda / 2$ involucran fermiones dentro de la región sombreada. Dado que la jerarquía de cutoffs es tal que $\Lambda \gg \lambda$, estos procesos representan sólo una pequeña corrección de superficie a los procesos de forward scattering que suceden dentro de los parches.

\subsubsection{Resolviendo la dinámica}

\section{Plan de acción}

Para calcular funciones de correlación debemos resolver las ecuaciones de Heisenberg para los operadores bosónicos $\left\{a_{\mathbf{S}}(\mathbf{q}), a_{\mathbf{S}}^{\dagger}(\mathbf{q})\right\}$. Esto es equivalente a diagonalizar el Hamiltoniano (3.34), lo cual si bien puede resolverse formalmente en forma cerrada-después de todo el Hamiltoniano es bilineal -, no puede obtenerse en forma explícita una solución analítica cerrada, dado que todos los parches están acoplados por la interacción. El empleo de esta solución explícita puede evitarse para nuestro propósito, debido a que es posible escribir la solución de las ecuaciones de movimiento exclusivamente en términos de las funciones de correlación retardadas de equilibrio de los campos bosónicos, algo que también resulta de la naturaleza bilineal del Hamiltoniano. Esas correlaciones retardadas pueden calcularse exactamente usando la formulación del problema bosónico en términos de una integral funcional de tiempo imaginario $[63,64,65]$.

\section{Detalles (y no tanto)}

Insertando la expresión Ec. (3.31) para las corrientes en cada parche en términos de los operadores bosónicos en el Hamiltoniano truncado (3.34), éste resulta $\left\{a_{\mathbf{S}}(\mathbf{q}), a_{\mathbf{S}}^{\dagger}(\mathbf{q})\right\}$,

$$
H^{F S}=\sum_{\mathbf{q}, \mathbf{S T}}\left[a_{\mathbf{S}}^{\dagger}(\mathbf{q}) V_{\mathbf{S T}}(\mathbf{q}) a_{\mathbf{T}}(\mathbf{q})+\left(a_{\mathbf{S}}^{\dagger}(\mathbf{q}) W_{\mathbf{S T}}(\mathbf{q}) a_{\mathbf{T}}^{\dagger}(-\mathbf{q})+\text { H.c. }\right)\right],
$$


en donde $V_{\text {ST }}$ y $W_{\text {ST }}$ describen el acoplamiento entre diferentes parches que proviene de la parte cinética y de interacción del Hamiltoniano truncado. Están dados por

$$
\begin{aligned}
& V_{\mathbf{S T}}=\delta_{\mathbf{S T}} 2 \theta\left(\hat{\mathbf{n}}_{\mathbf{S}} \cdot \mathbf{q}\right) \hat{\mathbf{n}}_{\mathbf{S}} \cdot \mathbf{q}+\frac{2 f(q) \Lambda}{(2 \pi)^{2}} \theta\left(\hat{\mathbf{n}}_{\mathbf{S}} \cdot \mathbf{q}\right) \theta\left(\hat{\mathbf{n}}_{\mathbf{T}} \cdot \mathbf{q}\right) \sqrt{\left(\hat{\mathbf{n}}_{\mathbf{S}} \cdot \mathbf{q}\right)\left(\hat{\mathbf{n}}_{\mathbf{T}} \cdot \mathbf{q}\right)}, \\
& W_{\mathbf{S T}}=\frac{f(q) \Lambda}{(2 \pi)^{2}} \theta\left(\hat{\mathbf{n}}_{\mathbf{S}} \cdot \mathbf{q}\right) \theta\left(-\hat{\mathbf{n}}_{\mathbf{T}} \cdot \mathbf{q}\right) \sqrt{\left(\hat{\mathbf{n}}_{\mathbf{S}} \cdot \mathbf{q}\right)\left(-\hat{\mathbf{n}}_{\mathbf{T}} \cdot \mathbf{q}\right)} .
\end{aligned}
$$

definimos el vector columna que contiene los operadores bosónicos

$$
\Phi=\left(\begin{array}{c}
a_{\mathbf{1}}(\mathbf{q}) \\
\ldots \\
a_{\mathbf{N}}(\mathbf{q}) \\
a_{\mathbf{1}}^{\dagger}(-\mathbf{q}) \\
\cdots \\
a_{\mathbf{N}}^{\dagger}(-\mathbf{q})
\end{array}\right),
$$

en donde $N$ es el número total de parches. Entonces, a menos de una constante aditiva irrelevante, el Hamiltoniano puede escribirse en forma matricial como

$$
H^{F S}=\sum_{q} q \Phi^{\dagger} \mathrm{H} \Phi
$$

en done $\mathrm{H}$ es una matriz hermítica de $2 N \times 2 N$. El Hamiltoniano ya es diagonal en el momento $q$ y para diagonalizarlo sólo debemos diagonalizar $\mathrm{H}$ para un dado $q$. Denotaremos la transformación que la diagonaliza de la siguiente manera:

$$
a_{\mathbf{S}}(\mathbf{q})=\sum_{\mathbf{T}} M_{\mathbf{S T}} \alpha_{\mathbf{T}}(\mathbf{q})+N_{\mathbf{S T}} \alpha_{\mathbf{T}}^{\dagger}(-\mathbf{q})
$$

en donde $\left\{\alpha_{\mathbf{T}}, \alpha_{\mathbf{T}}^{\dagger}\right\}$ es la base diagonal. A pesar de que la forma explícita de estas matrices puede deducirse, al menos para un número grande de parches [26], no será necesario hacerlo en nuestro caso. Notemos, sin embargo, que para que los operadores $\alpha$ satisfagan relaciones de conmutación canónicas, las matrices deben satisfacer:

$$
\begin{aligned}
& \sum_{\mathbf{T}} M_{\mathbf{S T}} M_{\mathbf{S}^{\prime} \mathbf{T}}-N_{\mathbf{S T}} N_{\mathbf{S}^{\prime} \mathbf{T}}=\delta_{\mathbf{S S}^{\prime}}, \\
& \sum_{\mathbf{T}} M_{\mathbf{S T}} N_{\mathbf{S}^{\prime} \mathbf{T}}-N_{\mathbf{S T}} M_{\mathbf{S}^{\prime} \mathbf{T}}=0, \\
& \sum_{\mathbf{T}} M_{\mathbf{T S}} M_{\mathbf{T S}^{\prime}}-N_{\mathbf{T S}} N_{\mathbf{T S}^{\prime}}=\delta_{\mathbf{S S}^{\prime}}, \\
& \sum_{\mathbf{T}} M_{\mathbf{T S}} N_{\mathbf{T S}^{\prime}}-N_{\mathbf{T S}} M_{\mathbf{T S}^{\prime}}=0 .
\end{aligned}
$$

Veamos entonces cómo reolver la dinámica sin obtener explícitamente las matrices $M_{\mathbf{S T}}$ y $N_{\text {ST }}$. El estado inicial es el estado fundamental $\left|\Psi_{0}\right\rangle$ del Hamiltoniano que se obtiene de $H^{F S}$ 
poniendo $f(q)=0$, es decir, el vacío de los operadores $a_{\mathbf{S}}(\mathbf{q})$. La forma general de la solución de las ecuaciones de Heisenberg para los operadores $a_{\mathbf{S}}$ toma la forma

$$
e^{i H t} a_{\mathbf{S}}(\mathbf{q}) e^{-i H t}=\sum_{\mathbf{T}} A_{\mathbf{S T}}(t) a_{\mathbf{T}}(\mathbf{q})+B_{\mathbf{S T}}(t) a_{\mathbf{T}}^{\dagger}(-\mathbf{q}) .
$$

en donde las matrices $A_{\mathbf{S T}}(t)$ y $B_{\mathbf{S T}}(t)$ pueden escribirse en términos de las matrices de transformación

$$
\begin{aligned}
& A_{\mathbf{S T}}(t)=\sum_{\mathbf{R}} M_{\mathbf{S R}} M_{\mathbf{T R}} e^{-i q S_{\mathbf{R}} t}-N_{\mathbf{S R}} N_{\mathbf{T R}} e^{i q S_{\mathbf{R}} t}, \\
& B_{\mathbf{S T}}(t)=\sum_{\mathbf{R}} N_{\mathbf{S R}} M_{\mathbf{T R}} e^{i q S_{\mathbf{R}} t}-M_{\mathbf{S R}} N_{\mathbf{T R}} e^{-i q S_{\mathbf{R}} t},
\end{aligned}
$$

y $S_{\mathbf{R}}$ son los autovalores de $\mathrm{H}$. Por otro lado, estas matrices pueden escribirse en términos de los correladores retardados de equilibrio. Para ver esto, primero notamos que

$$
\begin{aligned}
& A_{\mathbf{S T}}(t)=\left[a_{\mathbf{S}}(\mathbf{q}, t), a_{\mathbf{T}}^{\dagger}(\mathbf{q})\right], \\
& B_{\mathbf{S T}}(t)=\left[a_{\mathbf{S}}(\mathbf{q}, t), a_{\mathbf{T}}(-\mathbf{q})\right] .
\end{aligned}
$$

Dado que el Hamiltoniano es cuadrático, estos conmutadores son proporcionales al operador identidad, y, por lo tanto, podemos tomar su valor de expectación con respecto a cualquier estado debidamente normalizado. Dado que los operadores evolucionan de acuerdo con $H$ elegimos su estado fundamental $|\Psi\rangle$ para tomar el valor medio. Por lo tanto estas matrices pueden escribirse, para $t>0$, en términos de las siguientes funciones de correlación retardadas de equilibrio

$$
\begin{aligned}
& G_{\mathbf{S T}}^{\text {ret,eq }}(\mathbf{q}, t)=-i \theta(t)\left\langle\Psi\left|\left[a_{\mathbf{S}}(\mathbf{q}, t), a_{\mathbf{T}}^{\dagger}(\mathbf{q})\right]\right| \Psi\right\rangle, \\
& \tilde{G}_{\mathbf{S T}}^{\text {ret,eq }}(\mathbf{q}, t)=-i \theta(t)\left\langle\Psi\left|\left[a_{\mathbf{S}}(\mathbf{q}, t), a_{\mathbf{T}}(-\mathbf{q})\right]\right| \Psi\right\rangle .
\end{aligned}
$$

Las transformadas de Fourier de las funciones de correlación retardadas pueden ser calculadas mediante la continuación analítica de las funciones de Matsubara (temperatura finita, tiempo imaginario), las cuales, a su vez, pueden obtenerse en la formulación mediante integrales funcionales del problema bosónico en equilibrio Ec. (3.36) por medio de una transformación de Hubbard-Stratonovich, la cual no mostraremos explícitamente aquí (referimos al lector a [63] para un tratamiento detallado). De esta manera nos ahorramos el uso de la forma explícita de la transformación de Bogoliubov generalizada que diagonaliza el Hamiltoniano [26].

\subsubsection{Función de Green fermiónica}

Primero discutiremos cómo calcular la función de Green de los fermiones utilizando la solución a la dinámica que hemos descripto en la sección anterior y la identidad de bosonización. Los lectores que no estén interesados en los detalles técnicos pueden saltarse la sección del 
cálculo y pasar directamente a las siguientes en donde se discute las consecuencias físicas de los resultados.

La matriz densidad fermiónica de una partícula de no equilibrio se define como

$$
\mathscr{G}^{\text {neq }}(\mathbf{x}, t)=\left\langle\Psi_{0}\left|e^{i H t} \psi^{\dagger}(\mathbf{x}) \psi(\mathbf{0}) e^{i H t}\right| \Psi_{0}\right\rangle,
$$

la cual puede ser escrita como una suma sobre parches,

$$
\mathscr{G}^{\mathrm{neq}}(\mathbf{x}, t)=\sum_{\mathbf{S}} \mathscr{G}_{\mathbf{S}}^{\mathrm{neq}}(\mathbf{x}, t)
$$

con

$$
\mathscr{G}_{\mathbf{S}}^{\text {neq }}(\mathbf{x}, t)=\left\langle\Psi_{0}\left|e^{i H t} \psi_{\mathbf{S}}^{\dagger}(\mathbf{x}) \psi_{\mathbf{S}}(\mathbf{0}) e^{i H t}\right| \Psi_{0}\right\rangle,
$$

La función análoga en equilibrio pude definirse como:

$$
\mathscr{G}_{\mathbf{S}}^{\mathrm{eq}}(\mathbf{x})=\left\langle\Psi\left|\psi_{\mathbf{S}}^{\dagger}(\mathbf{x}) \psi_{\mathbf{S}}(\mathbf{0})\right| \Psi\right\rangle .
$$

El cálculo puede llevarse a cabo usando la identidad de bosonización multidimensional Eq. (3.24), la cual repetimos aquí para facilidad de lectura

$$
\psi_{\mathbf{S}}(\mathbf{x})=e^{i \mathbf{k}_{\mathbf{s}} \cdot \mathbf{x}} \sqrt{\frac{\Omega}{V a}} \exp \left[i \frac{1}{\sqrt{\Omega}} \phi_{\mathbf{S}}(\mathbf{x})\right],
$$

en donde $a=1 / \lambda$ es el cutoff ultravioleta y el campo bosónico viene dado por

$$
\phi_{\mathbf{S}}(\mathbf{x})=i \sum_{\mathbf{q}, \hat{\mathbf{n}}_{\mathbf{S}} \cdot \mathbf{q}>0} \frac{a_{\mathbf{S}}^{\dagger}(\mathbf{q}) e^{-i \mathbf{q} \cdot \mathbf{x}}-a_{\mathbf{S}}(\mathbf{q}) e^{i \mathbf{q} \cdot \mathbf{x}}}{\sqrt{\hat{\mathbf{n}}_{\mathbf{S}} \cdot \mathbf{q}}} .
$$

Primero calcularemos el correlador en equilibrio para poder compararlo luego con el de no equilibrio y para introducir algunas herramientas necesarias. Usando resultados conocidos sobre valores de expectación gaussianos (completamente análogos a los usados en el capítulo anterior en $D=1$ ) encontramos que el correlador es la exponencial de

$$
\begin{aligned}
\left\langle\phi_{\mathbf{S}}(\mathbf{x}) \phi_{\mathbf{S}}(\mathbf{0})\right\rangle_{\mathrm{eq}}-\left\langle\phi_{\mathbf{S}}(\mathbf{x})^{2}\right\rangle_{\mathbf{e q}} & =\sum_{\mathbf{q}, \hat{\mathbf{n}}_{\mathbf{S}} \cdot \mathbf{q}>0} \frac{1}{\hat{\mathbf{n}}_{\mathbf{S}} \cdot \mathbf{q}} \\
\times & {\left[\left(e^{i \mathbf{q} \cdot \mathbf{x}}-1\right)\left\langle a_{\mathbf{S}}(\mathbf{q}) a_{\mathbf{S}}^{\dagger}(\mathbf{q})\right\rangle_{\mathrm{eq}}+\left(e^{-i \mathbf{q} \cdot \mathbf{x}}-1\right)\left\langle a_{\mathbf{S}}^{\dagger}(\mathbf{q}) a_{\mathbf{S}}(\mathbf{q})\right\rangle_{\mathrm{eq}}\right], }
\end{aligned}
$$

en donde hemos introducido la notación $\langle\ldots\rangle_{\mathrm{eq}}=\langle\Psi|\ldots| \Psi\rangle$. Usando las propiedades de las matrices de transformación (3.41)-(3.44) se muestra que

$$
\left\langle a_{\mathbf{S}}(\mathbf{q}) a_{\mathbf{S}}^{\dagger}(\mathbf{q})\right\rangle_{\mathrm{eq}}=\sum_{\mathbf{R}}\left(M_{\mathbf{S R}}\right)^{2}=1+\sum_{\mathbf{R}}\left(N_{\mathbf{S R}}\right)^{2}=1+\left\langle a_{\mathbf{S}}^{\dagger}(\mathbf{q}) a_{\mathbf{S}}(\mathbf{q})\right\rangle_{\mathrm{eq}},
$$


de manera que

$\left\langle\phi_{\mathbf{S}}(\mathbf{x}) \phi_{\mathbf{S}}(\mathbf{0})\right\rangle_{\mathrm{eq}}-\left\langle\phi_{\mathbf{S}}(\mathbf{x})^{2}\right\rangle_{\mathrm{eq}}=\sum_{\mathbf{q}, \hat{\mathbf{n}}_{\mathbf{S}} \cdot \mathbf{q}>0} \frac{1}{\hat{\mathbf{n}}_{\mathbf{S}} \cdot \mathbf{q}}\left(e^{i \mathbf{q} \cdot \mathbf{x}}-1\right)+\sum_{\mathbf{q}, \hat{\mathbf{n}}_{\mathbf{S}} \cdot \mathbf{q}>0} \frac{1}{\hat{\mathbf{n}}_{\mathbf{S}} \cdot \mathbf{q}} 2\left\langle a_{\mathbf{S}}^{\dagger}(\mathbf{q}) a_{\mathbf{S}}(\mathbf{q})\right\rangle_{\mathrm{eq}}(\cos (\mathbf{q} \cdot \mathbf{x})-1)$.

Después de exponenciar, el primer término produce el correlador de fermiones libres [63]

$$
\mathscr{G}_{\mathbf{S}}^{0}(\mathbf{x})=\frac{i \Lambda}{(2 \pi)^{2}} \frac{e^{i k_{F} \hat{\mathbf{n}}_{\mathbf{s}} \cdot \mathbf{x}}}{\hat{\mathbf{n}}_{\mathbf{S}} \cdot \mathbf{x}} W_{\Lambda}\left(\left|\hat{\mathbf{n}}_{\mathbf{S}} \times \mathbf{x}\right|\right),
$$

en donde $W_{\Lambda}\left(\left|\hat{\mathbf{n}}_{\mathbf{S}} \times \mathbf{x}\right|\right)$ es una función de cutoff que se hace nula para $\left|\hat{\mathbf{n}}_{\mathbf{S}} \times \mathbf{x}\right| \Lambda \gtrsim 1$ y alcanza el valor unidad para $\left|\hat{\mathbf{n}}_{\mathbf{S}} \times \mathbf{x}\right|=0$. El segundo término es la corrección por la interacción $Z_{\mathbf{S}}^{\mathrm{eq}}(\mathbf{x})$ que analizaremos más abajo. Usando el fomralismo de Matsubara y la transformación de Hubbard-Stratonovich podemos hallar una expresión para $\left\langle a_{\mathbf{S}}^{\dagger}(\mathbf{q}) a_{\mathbf{S}}(\mathbf{q})\right\rangle_{e q}$ a partir del valor de expectación ordenado temporalmente en tiempo maginario

$$
\begin{aligned}
\left\langle a_{\mathbf{S}}^{\dagger}(\mathbf{q}) a_{\mathbf{S}}(\mathbf{q})\right\rangle_{\mathrm{eq}} & =\lim _{\tau \rightarrow i 0^{-}}\left\langle T_{\tau} a_{\mathbf{S}}(\mathbf{q}, \tau) a_{\mathbf{S}}^{\dagger}(\mathbf{q}, 0)\right\rangle \\
& =\left|\hat{\mathbf{n}}_{\mathbf{S}} \cdot \mathbf{q}\right| \frac{f_{0} \Lambda^{2}}{(2 \pi)^{4}} \sum_{\mathbf{T}} \frac{\mathscr{P} V\left(-\hat{\mathbf{n}}_{\mathbf{T}} \cdot \mathbf{q}\right)}{\left(\hat{\mathbf{n}}_{\mathbf{S}} \cdot \mathbf{q}-\hat{\mathbf{n}}_{\mathbf{T}} \cdot \mathbf{q}\right)^{2}}+\mathscr{O}\left(f_{0}^{3}\right),
\end{aligned}
$$

en donde $\mathscr{P V}$ indica que en el límite de número de parches grande, en donde las sumas sobre parches pueden reemplazarse por integrales, debemos tomar el valor principal, una característica matemática que también surge en el tratamiento de equilibrio [26]. Encontramos

$$
Z_{\mathbf{S}}^{\mathrm{eq}}(\mathbf{x})=\exp \left[\frac{1}{\Omega} \frac{2 \Lambda^{2}}{(2 \pi)^{4}} f_{0}^{2} \sum_{\mathbf{q}, \mathbf{n}_{\mathbf{S}} \cdot \mathbf{q}>0, \mathbf{T}} \frac{\mathscr{P} V\left(-\hat{\mathbf{n}}_{\mathbf{T}} \cdot \mathbf{q}\right)}{\left(\hat{\mathbf{n}}_{\mathbf{S}} \cdot \mathbf{q}-\hat{\mathbf{n}}_{\mathbf{T}} \cdot \mathbf{q}\right)^{2}}(\cos (\mathbf{q} \cdot \mathbf{x})-1)\right] .
$$

En la literatura, las integrales de momento son calculadas usando un sistema de coordenadas cartesiano $\left(q_{\perp}, q_{\|}\right)$fijo en el parche $\mathbf{S}[26,63]$

$$
\begin{aligned}
q_{\perp} & =\hat{\mathbf{n}}_{\mathbf{S}} \cdot \mathbf{q}, \\
q_{\|} & =\sqrt{|\mathbf{q}|^{2}-\left(\hat{\mathbf{n}}_{\mathbf{S}} \cdot \mathbf{q}\right)^{2}} .
\end{aligned}
$$

Usaremos un método alternativo que hallamos más conveniente para el cálculo fuera de equilibrio que consiste en usar coordenadas polares en el mismo sistema de referencia

$$
\begin{aligned}
q & =\sqrt{q_{\perp}^{2}+q_{\|}^{2}}, \\
\tan \left(\theta_{\mathbf{S}}\right) & =\frac{q_{\perp}}{q_{\|}} .
\end{aligned}
$$


Nótese que el cutoff de las integrales radiales en q será la transferencia típica de momentos de la interacción $q_{c}$ [80]. Obtenemos

$$
\begin{aligned}
\sum_{\mathbf{q}, \hat{\mathbf{n}}_{\mathbf{S}} \cdot \mathbf{q}>0, \mathbf{T}} \frac{\mathscr{P V}\left(-\hat{\mathbf{n}}_{\mathbf{T}} \cdot \mathbf{q}\right)}{\left(\hat{\mathbf{n}}_{\mathbf{S}} \cdot \mathbf{q}-\hat{\mathbf{n}}_{\mathbf{T}} \cdot \mathbf{q}\right)^{2}}(\cos (\mathbf{q} \cdot \mathbf{x})-1) & =\frac{L^{2}}{(2 \pi)^{2}} \sum_{\mathbf{T}} \int_{-\pi / 2}^{\pi / 2} d \theta_{\mathbf{S}} \frac{\mathscr{P V}\left(-\cos \left(\theta_{\mathbf{T}}\right)\right)}{\cos \left(\theta_{\mathbf{S}}-\varphi_{\mathbf{S}}\right)\left(\cos \left(\theta_{\mathbf{S}}\right)-\cos \left(\theta_{\mathbf{T}}\right)\right)^{2}} \\
& \times\left[\frac{1}{|\mathbf{x}|} \sin \left(|\mathbf{x}| q_{c}\left(\cos \left(\theta_{\mathbf{S}}-\varphi_{\mathbf{S}}\right)\right)\right)-q_{c}\right],
\end{aligned}
$$

en donde $\varphi_{\mathbf{S}}$ es el ángulo entre el vector $\mathbf{x}$ y $\hat{\mathbf{n}}_{\mathbf{S}}$. Para distancias largas, $|\mathbf{x}| \gg q_{c}^{-1}$, el término oscilatorio se promedia a cero. Nótese entonces que toda la información sobre el índice de parche se pierde dado que el término restante tiene todos los índices de parche contraidos. Finalmente obtenemos

$$
\left\langle\psi_{\mathbf{S}}^{\dagger}(\mathbf{x}) \psi_{\mathbf{S}}(\mathbf{0})\right\rangle_{e q}=\mathscr{G}_{\mathbf{S}}^{0}(\mathbf{x}) \exp \left[-\frac{2 f_{0}^{2} N(0)}{(2 \pi)^{3}} q_{c} c+\mathscr{O}\left(f_{0}^{3}\right)\right],
$$

en donde

$$
c=\mathscr{P V} \int_{0}^{2 \pi} d \theta_{\mathbf{T}} \int_{-\pi / 2}^{\pi / 2} d \theta_{\mathbf{S}} \frac{\left(-\cos \left(\theta_{\mathbf{T}}\right)\right)}{\left(\cos \left(\theta_{\mathbf{S}}\right)-\cos \left(\theta_{\mathbf{T}}\right)\right)^{2}}=\int_{\pi / 2}^{3 \pi / 2} d \theta_{\mathbf{T}} \int_{-\pi / 2}^{\pi / 2} d \theta_{\mathbf{S}} \frac{\left|\cos \left(\theta_{\mathbf{T}}\right)\right|}{\left(\cos \left(\theta_{\mathbf{S}}\right)-\cos \left(\theta_{\mathbf{T}}\right)\right)^{2}} \simeq 5 .
$$

Nótese que hemos reemplazado, sólo por conveniencia, las sumas sobre parchesT presentes en Ec. (3.67) por una integral, lo cual es una aproximación válida para $N$ grande. En la Ec. (3.70) el factor exponencial que multiplica al correlador libre no tiene índice de parche y por lo tanto se factoriza de la suma final sobre parches (Ec. (3.51)) necesaria para obtener el correlador físico. Concluimos que el correlador para los fermiones físicos viene dado por:

$$
\left\langle\psi^{\dagger}(\mathbf{x}) \psi(\mathbf{0})\right\rangle_{e q}=\mathscr{G}^{0}(\mathbf{x}) \exp \left[-\frac{2 f_{0}^{2} N(0)}{(2 \pi)^{3}} q_{c} c+\mathscr{O}\left(f_{0}^{3}\right)\right],
$$

en donde $\mathscr{G}^{0}(\mathbf{x})$ es el correlador global libre. Entonces identificamos el factor exponencial con $Z^{\mathrm{eq}}$, el residuo de cuasipartícula en equilibrio. Desarrollando en potencias de $f_{0}$ obtenemos

$$
Z^{\mathrm{eq}}=1-\frac{2 f_{0}^{2} N(0)}{(2 \pi)^{3}} q_{c} c+\mathscr{O}\left(f_{0}^{3}\right)
$$

lo cual es consistente con un cálculo perturbativo usual en términos de los grados de libertad fermiónicos.

Ahora mostraremos los detalles del cálculo del correlador fuera de equilibrio. A partir de la 
Ec. (3.45) y el hecho de que $\left|\Psi_{0}\right\rangle$ es el vacío de los operadores $a_{\mathbf{S}}$, se verifica que

$$
\left\langle\phi_{\mathbf{S}}(\mathbf{x}, t) \phi_{\mathbf{S}}(\mathbf{0}, t)\right\rangle_{\text {neq }}-\left\langle\phi_{\mathbf{S}}(\mathbf{x})^{2}\right\rangle_{\text {neq }}=\sum_{\mathbf{q}, \hat{\mathbf{n}} \mathbf{s} \cdot \mathbf{q}>0, \mathbf{T}} \frac{1}{\hat{\mathbf{n}}_{\mathbf{S}} \cdot \mathbf{q}}\left[\left(e^{i \mathbf{q} \cdot \mathbf{x}}-1\right)\left|A_{\mathbf{S T}}(t)\right|^{2}+\left(e^{-i \mathbf{q} \cdot \mathbf{x}}-1\right)\left|B_{\mathbf{S T}}(t)\right|^{2}\right],
$$

en donde $\langle\ldots\rangle_{\text {neq }}=\left\langle\Psi_{0}|\ldots| \Psi_{0}\right\rangle$. Usando las propiedades de las matrices de Bogoliubov generalizadas $M_{\mathbf{S T}}$ y $N_{\mathbf{S T}}$, Ec. (3.41), encontramos que

$$
\left\langle\phi_{\mathbf{S}}(\mathbf{x}, t) \phi_{\mathbf{S}}(\mathbf{0}, t)\right\rangle_{\text {neq }}-\left\langle\phi_{\mathbf{S}}(\mathbf{x})^{2}\right\rangle_{\text {neq }}=\sum_{\mathbf{q}, \hat{\mathbf{n}}_{\mathbf{S}} \cdot \mathbf{q}>0} \frac{1}{\hat{\mathbf{n}}_{\mathbf{S}} \cdot \mathbf{q}}\left(e^{i \mathbf{q} \cdot \mathbf{x}}-1\right)+\sum_{\mathbf{q}, \hat{\mathbf{n}_{\mathbf{S}} \cdot \mathbf{q}>0, \mathbf{T}}} 2(\cos (\mathbf{q} \cdot \mathbf{x})-1)\left|B_{\mathbf{S T}}(t)\right|^{2} .
$$

Como antes, después de exponenciar, el primer término produce el correlador libre (la condición inicial), mientras que el segundo corresponde a la corrección producida por el encendido repentino de la interacción $Z_{\mathbf{S}}^{\text {neq }}(\mathbf{x}, t)$. Dado que $\left|B_{\mathbf{S T}}(t)\right|^{2}=\left|\tilde{G}_{\mathbf{S T}}^{\text {ret,eq }}(\mathbf{q}, t)\right|^{2}$, debemos considerar la función de correlación retardada de equilibrio de los bosones. Ésta puede obtenerse como la continuación analítica de la función de Green en tiempo imaginario utilizada antes para el cálculo de equilibrio. Obtenemos

$$
\left|\tilde{G}_{\mathbf{S T}}^{\mathrm{ret}, \mathrm{eq}}(\mathbf{q}, t)\right|^{2}=\frac{2 f^{2}(q) \Lambda^{2}}{(2 \pi)^{4}} \mathscr{P V} \frac{\left|\hat{\mathbf{n}}_{\mathbf{S}} \cdot \mathbf{q} \| \hat{\mathbf{n}}_{\mathbf{T}} \cdot \mathbf{q}\right|}{\left(\hat{\mathbf{n}}_{\mathbf{S}} \cdot \mathbf{q}-\hat{\mathbf{n}}_{\mathbf{T}} \cdot \mathbf{q}\right)^{2}}\left(1-\cos \left[\left(\hat{\mathbf{n}}_{\mathbf{S}} \cdot \mathbf{q}-\hat{\mathbf{n}}_{\mathbf{T}} \cdot \mathbf{q}\right) t\right]\right)+\mathscr{O}\left(f_{0}^{3}\right),
$$

en donde hemos mostrado sólo el primer orden en la interacción. Como era de esperarse, la dependencia temporal de $\tilde{G}_{\mathbf{S T}}^{\text {ret,eq }}(\mathbf{q}, t)$ implica que $Z_{\mathbf{S}}^{\text {neq }}(\mathbf{x}, 0)=1$. La dependencia temporal completa, que puede obtenerse resolviendo las integrales en momento usando las coordenadas polares, será discutida más adelante.

\subsubsection{Estado estacionario}

Recapitulando, podemos escribir a la función de correlación después del quench como $\mathscr{G}_{\mathbf{S}}^{\text {neq }}(\mathbf{x}, t)=\mathscr{G}_{\mathbf{S}}^{0}(\mathbf{x}) Z_{\mathbf{S}}^{\text {neq }}(\mathbf{x}, t)$, en donde $\mathscr{G}_{\mathbf{S}}^{0}(\mathbf{x})$ es el correlador de parche libre (la condición incial), $\mathrm{y}$

$$
Z_{\mathbf{S}}^{\mathrm{neq}}(\mathbf{x}, t)=\exp \left[\frac{1}{\Omega} \sum_{\mathbf{q}, \hat{\mathbf{n}}_{\mathbf{S}} \cdot \mathbf{q}>0, \mathbf{T}} \frac{\left|\tilde{G}_{\mathbf{S T}}^{\mathrm{ret}, \mathrm{eq}}(\mathbf{q}, t)\right|^{2}}{\hat{\mathbf{n}}_{\mathbf{S}} \cdot \mathbf{q}} \times 2(\cos (\mathbf{q} \cdot \mathbf{x})-1)\right],
$$

en donde $\tilde{G}_{\mathbf{S T}}^{\text {ret,eq }}(\mathbf{q}, t)$ es la función de correlación anómala en equilibrio de los bosones, cuya expresión a orden más bajo en $f_{0}$ está en la Ec. (3.74), y $\mathbf{n}$ es el vector unitario normal a la superficie de Fermi en el parche $\mathbf{S}$. Para tiempos largos, el factor oscilatorio en $\tilde{G}_{\mathbf{S T}}^{\text {ret,eq }}(\mathbf{q}, t)$ se promedia a cero en la integral Ec. (3.75) debido al dephasing y se alcanza así un estado estacionario. Insertando el factor independiente del tiempo de (3.74) en (3.73) y comparando 
con (3.62) encontramos que

$$
\lim _{t \rightarrow \infty} \ln \left[Z_{\mathbf{S}}^{\mathrm{neq}}(\mathbf{x}, t)\right]=2 \ln \left[Z_{\mathbf{S}}^{\mathrm{eq}}(\mathbf{x})\right]+\mathscr{O}\left(f_{0}^{3}\right) .
$$

Para obtener el correlador de los fermiones físicos (tanto en equilibrio como fuera de equilibrio) aún debemos realizar la suma final sobre parches [Ec. (3.51)], una perspectiva algo atemorizante. Afortunadamente, la tarea puede simplificarse: debido a la presencia de la función $W_{\Lambda}$ y del factor exponencial $e^{i k_{F} \hat{\mathbf{n}}_{\mathbf{S}} \cdot \mathbf{x}}$ en $\mathscr{G}_{\mathbf{S}}^{0}(\mathbf{x})$ la suma sobre parches estará dominada por contribuciones provenientes de parches para los cuales $\varphi_{\mathbf{S}}=\arccos \left(\frac{\hat{\mathbf{n}}_{\mathbf{s}} \cdot \mathbf{x}}{|\mathbf{x}|}\right)$ es cercano a cero. Es más, dado que $Z_{\mathbf{S}}^{\text {neq }}(\mathbf{x}, t)$ varía lentamente alrededor de $\varphi_{\mathbf{S}}=0$, la función de correlación global puede aproximarse como

$$
\mathscr{G}^{\text {neq }}(x, t)=\mathscr{G}^{0}(x) Z^{\text {neq }}(x, t),
$$

en donde $\mathscr{G}^{0}(x)$ es el correlador libre, $x=|\mathbf{x}|$ y $Z^{\text {neq }}(x, t)=\left.Z_{\mathbf{S}}^{\text {neq }}(\mathbf{x}, t)\right|_{\varphi_{\mathrm{S}}=0}$, y una expresión análoga para el propagador en equilibrio. Entonces, la relación (3.76) implica una relación análoga para los correladores globales:

$$
\lim _{t \rightarrow \infty} \ln \left[Z^{\mathrm{neq}}(x, t)\right]=2 \ln \left[Z^{\mathrm{eq}}(x)\right]+\mathscr{O}\left(f_{0}^{3}\right) .
$$

Tal como está definida, puede mostrarse que el límite $x \rightarrow \infty$ de $Z(x)$ (tanto en equilibrio como fuera de equilibrio) es $Z$, el valor del salto en el momento de Fermi de la distribución de momentos. Por lo tanto, de la Ec. (3.78) se puede deducir una relación entre la discontinuidad en equilibrio y en el estado estacionario después del quench: $Z^{\text {neq }} \simeq\left(Z^{\mathrm{eq}}\right)^{2}$. Recordando que $Z^{\mathrm{eq}}=1-\mathscr{O}\left(f_{0}^{2}\right)$, volvemos a encontrar el resultado obtenido usando teoría de perturbaciones $1-Z^{\text {st }}=2\left(1-Z^{\mathrm{eq}}\right)$.

Podemos ver esto de otra manera. Exponenciando la Ec. (3.78) y dsarrollando en potencias de la interacción podemos obtener una relación simple entre las distribuciones de momento en el estado estacionario, $n^{\text {st }}(k)$, y en el estado fudamental (equilibrio), $n^{\mathrm{eq}}(k)$,

$$
2\left[n^{\mathrm{eq}}(k)-n_{0}^{\mathrm{eq}}(k)\right]=n^{\mathrm{st}}(k)-n_{0}^{\mathrm{eq}}(k)+\mathscr{O}\left(f_{0}^{3}\right),
$$

en donde $n_{0}^{\mathrm{eq}}(k)=\theta\left(k_{F}-k\right)$. El resultado para la discontinuidad en el momento de Fermi se obtiene evaluando el resultado de la distribución de momentos en $k=k_{F}$. Un resultado similar fue obtenido en Refs. [95, 96] para el modelo de Hubbard en condiciones un tanto diferentes (interacciones de corto alcance entre fermiones con espín).

El resultado (3.79) para la distribución de momentos tiene implicancias para la distribución de energía en el estado pretermalizado. De hecho, este resultado implica que, a orden dominante en la interacción, la energía cinética total en el estado estacionario $E_{\text {kin }}^{\text {neq }}=\left\langle\Psi_{0}\left|H_{0}\right| \Psi_{0}\right\rangle$, es el doble que la energía en el estado fundamental de $H, E_{\text {kin }}^{\mathrm{eq}}=\left\langle\Psi\left|H_{0}\right| \Psi\right\rangle$ [95]. Esto implica que a orden dominante en la interacción la energía inyectada en el sistema por el quench, $E^{\text {ex }}=E^{\text {neq }}-E^{\text {eq }}$, en donde $E^{\text {neq }}=\left\langle\Psi_{0}|H| \Psi_{0}\right\rangle=0$ y $E^{\text {eq }}=\langle\Psi|H| \Psi\rangle$, se transforma en energía 
cinética en el estado estacionario, algo que también se observa en [95]. Determinar que este sea el valor final (térmico) de la energía cinética luego del quench está más allá de las posibilidades de nuestro método y del método perturbativo, los cuales son válidos a tiempos cortos y no pueden capturar la dinámica de tiempos largos. Sin embargo, como fruto de la relajación por dephasing generalmente algunos observables alcanzan su valor térmico final, siendo la energía cinética del sistema el ejemplo más prominente de esto [8]. En efecto, en el capítulo siguiente veremos en un ejemplo en $D=1$ la energía cinética del sistema alcanza su valor térmico en la escala de tiempo de la pretermalización.

En resumen, el tratamiento con bosonización nos permite reobtener el resultado sobre el estado estacionario que surge del tratamiento perturbativo, mas ahora resulta claro que los modos cuasilibres detrás de la relajación por dephasing están relacionados con los bosones que diagonalizan el Hamiltoniano truncado $H^{F S}$ que describe la dinámica a tiempos cortos, cerca de la superficie de Fermi. Como se ha indicado antes, estos modos bosónicos representan las fluctuaciones en la forma de la superficie de Fermi [25].

\subsubsection{Ensamble de Gibbs generalizado}

Si bien en sistemas integrables esperamos que el estado final de la evolución esté descripto exactamente por un GGE, podemos esperar que en la relajación de sistemas casi integrables (que deben relajar al equilibrio térmico ya que no poseen cantidades conservadas no triviales) surjan estados estacionarios que precedan al estado térmico que estén relacionados, justamente, con la cercanía de un punto integrable. Recientemente la posibilidad de describir tales estados estacionarios de no equilibrio en términos de un ensamble tipo GGE ha recibido mucha antención $[56,78,43]$. El único enfoque que se ha utilizado hasta el momento para probar este resultado es obtener ciertas cantidades casi conservadas en un sistema con interacciones débiles perturbando los operadores de ocupación de los modos de momento $c_{\mathbf{k}}^{\dagger} c_{\mathbf{k}}$, las constantes de movimiento exactas del sistema libre [78, 43]. El GGE construido con estas cantidades cuasiconservadas puede reproducir algunas funciones de correlación en el estado cuasiestacionario que surge en la relajación del sistema luego de un encendido repentino de las interacciones débiles.

En este contexto, quizás la mayor ventaja que provee el tratamiento con bosonización es que, al identificar los modos cuasilibres que dominan la relajación a tiempos cortos, permite construir de inmediato una descripción estadística en términos de un GGE del estado estacionario, de manera completamente análoga a lo discutido para el sistema en $D=1$ en el capítulo anterior. En particular, como fue discutido en el capítulo anterior, el hecho de que el Hamiltoniano truncado es un bilineal bosónico implica que todas las correlaciones en el estado estacionario están descriptas por un GGE [31,3]. Si denotamos $\operatorname{con}\left\{\alpha_{l}(\mathbf{q}), \alpha_{l}^{\dagger}(\mathbf{q})\right\}$ a la base bosónica que diagonaliza el hamiltoniano Ec. (3.34), la matriz densidad del GGE puede escribirse como

$$
\rho_{\mathrm{GGE}}=\frac{1}{Z_{\mathrm{GGE}}} \exp \left[\sum_{l, \mathbf{q}} \lambda_{l}(\mathbf{q}) I_{l}(\mathbf{q})\right],
$$


en donde $I_{l}(\mathbf{q})=\alpha_{l}^{\dagger}(\mathbf{q}) \alpha_{l}(\mathbf{q})$ son las cantidades conservadas, $Z_{\mathrm{GGE}}=\operatorname{Tr}\left[\rho_{\mathrm{GGE}}\right]$ y los multiplicadores de Lagrange $\lambda_{l}(\mathbf{q})$ se obtienen a partir de las condiciones iniciales, $\left\langle I_{l}(\mathbf{q})\right\rangle_{t=0}=$ $\left\langle\Psi_{0}\left|I_{l}(\mathbf{q})\right| \Psi_{0}\right\rangle=\operatorname{Tr}\left[\rho_{\mathrm{GGE}} I_{l}(\mathbf{q})\right]$. También peude verificarse explícitamente, usando las propiedades de las matrices de Bogoliubov generalizadas (3.41) que la matriz densidad (3.80) reproduce el correlador fermiónico en el estado estacionario. También es importante notar que las cantidades conservadas pueden ser refermionizadas, ya que pueden ser expresadas como una combinación lineal de productos de dos densidades de parche $J_{\mathbf{S}}(\mathbf{q})$.

Para contrastar con el método utilizado en las Refs. [78, 43], hacemos notar que no hemos construido un GGE con algunas cantidades cuasiconservadas obtenidas mediante teoría de perturbaciones y verificado que reproduzcan las correlaciones en el estado estacionario, sino que hemos demostrado que la dinámica a tiempos cortos está descripta por un modelo efectivo que es integrable y que, por lo tanto, el estado estacionario está descripto por el GGE asociado con ese modelo integrable. A tiempos largos el modelo efectivo deja de tener validez y el sistema relaja al estado térmico correspondiente.

\subsubsection{Dinámica}

Es bien conocido que despupués de un quench en sistemas en $D=1$ las correlaciones se propagan a una velocidad finita dando lugar al efecto horizonte [16], el cual hemos discutido oportunamente en el capítulo anterior. Sólo recientemente se ha comenzado a explorar este fenómeno en dimensiones superiores [22].

En nuestro caso, a partir de las Ecs. (3.73),(3.74), podemos mostrar analíticamente que existe un efecto horizonte en las correlaciones. Notemos primero que:

$$
\begin{aligned}
(\cos (\mathbf{q} \cdot \mathbf{x})-1)\left(1-\cos \left[\left(\hat{\mathbf{n}}_{\mathbf{S}} \cdot \mathbf{q}-\hat{\mathbf{n}}_{\mathbf{T}} \cdot \mathbf{q}\right) t\right]\right)= & -\frac{1}{2}\left\{\cos \left[\mathbf{q} \cdot\left(\mathbf{x}+\left(\hat{\mathbf{n}}_{\mathbf{S}}-\hat{\mathbf{n}}_{\mathbf{T}}\right) t\right)\right]+\cos \left[\mathbf{q} \cdot\left(\mathbf{x}-\left(\hat{\mathbf{n}}_{\mathbf{S}}-\hat{\mathbf{n}}_{\mathbf{T}}\right) t\right)\right]\right\} \\
& -1+\cos (\mathbf{q} \cdot \mathbf{x})+\cos \left[\left(\hat{\mathbf{n}}_{\mathbf{S}} \cdot \mathbf{q}-\hat{\mathbf{n}}_{\mathbf{T}} \cdot \mathbf{q}\right) t\right] .
\end{aligned}
$$

Podemos entonces corroborar que, si $|\mathbf{x}| \gg\left|\hat{\mathbf{n}}_{\mathbf{S}}-\hat{\mathbf{n}}_{\mathbf{T}}\right| v_{F} t$ para todo $\mathbf{S}$ y $\mathbf{T}$, es decir, si $|\mathbf{x}| \gg 2 v_{F} t$, entonces, a primer orden en $\frac{2 v_{F} t}{|\mathbf{x}|}$, es posible despreciar la dependencia espacial de $Z_{\mathbf{S}}^{\text {neq }}(\mathbf{x}, t)$. Es importante notar entonces que luego de resolver las integrales en momentos en la Ec. (3.73), el índice de parche de $Z_{\mathbf{S}}^{\text {neq }}(\mathbf{x}, t)$ sólo está presente a través de su dependencia espacial (ver la discusión después de la Ec. (3.67)). Fuera del cono de luz, $|\mathbf{x}| \gg 2 v_{F} t$, la corrección por el quench es por lo tanto aproximadamente la misma para todos los parches: $Z_{\mathbf{S}}^{\text {neq }}(\mathbf{x}, t) \approx Z^{\text {neq }}(t)$. La función de correlación de los fermiones físicos es entonces $\mathscr{G}^{\text {neq }}(x, t) \approx \mathscr{G}^{0}(x) Z^{\text {neq }}(t)$, es decir, las correlaciones guardan la misma dependencia espacial que las iniciales y difieren de éstas en un factor que depende del tiempo. Este factor define el residuo de cuasipartícula instantáneo $Z^{\text {neq }}(t)$, el cual será discutido más abajo. En el límite opuesto, $|\mathbf{x}| \ll 2 v_{F} t$, podemos despreciar la dependencia temporal y las correlaciones del estado estacionario dominan: $Z^{\text {neq }}(x, t) \approx \lim _{t \rightarrow \infty} Z^{\text {neq }}(x, t)$. Concluimos que, en la escala de tiempos de la pretermalización, las correlaciones viajan isotrópicamente con la velocidad de Fermi de los fermiones 


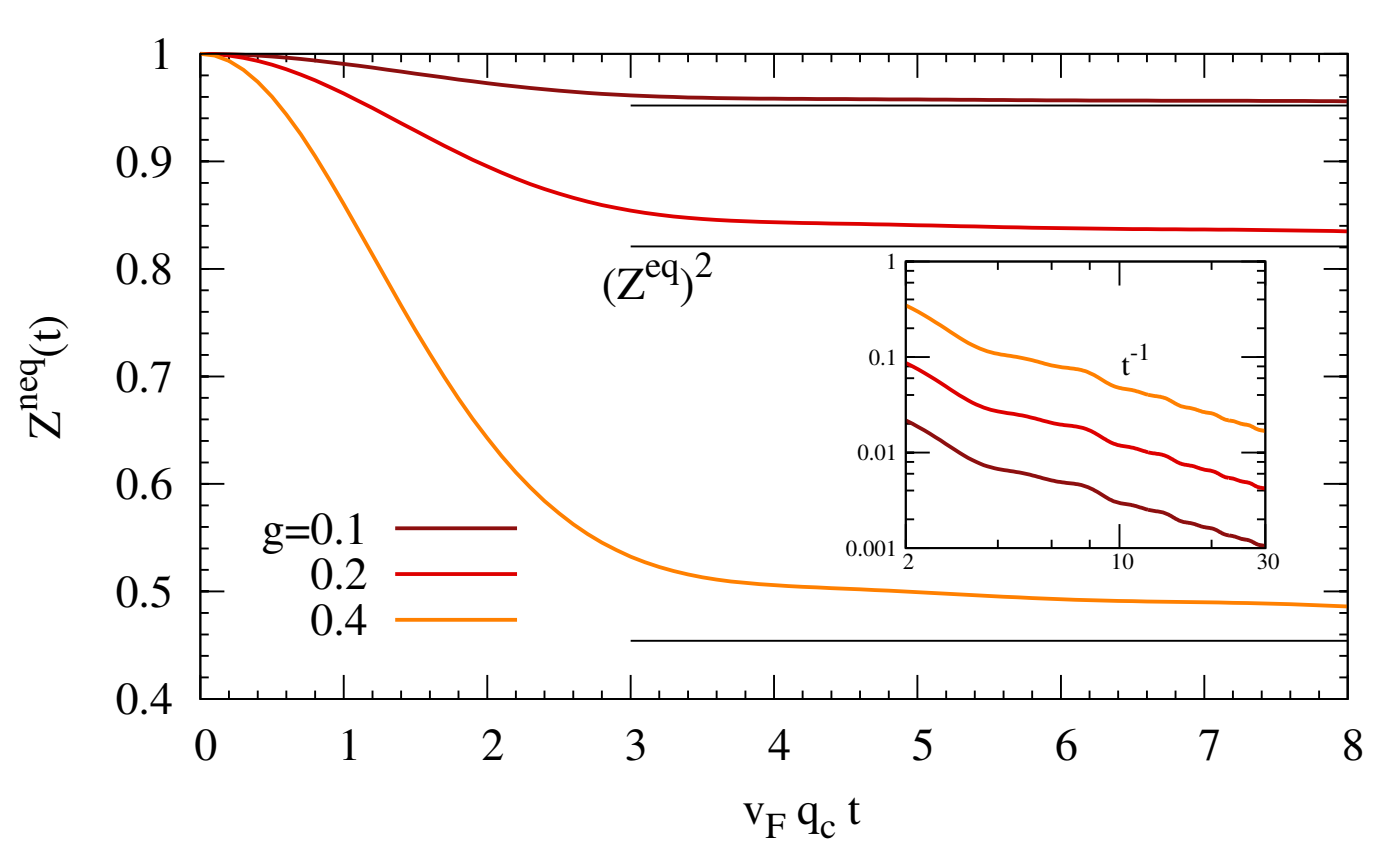

Figura 3.4: Discontinuidad en la distribución de momentos en el momento de Fermi, $Z^{\text {neq }}(t)$, como función de $t$ para varios valores de $g=f_{0} \sqrt{k_{F} q_{c}} /\left(2 \pi^{2} v_{F}\right) . Z^{\text {neq }}(t)$ exhibe un decaimiento gaussiano a tiempos cortos. Satura asintóticamente a un valor finito $Z^{\text {st }} \simeq\left(Z^{\mathrm{eq}}\right)^{2}$ (líneas horizontales) en el estado pretermalizado. Inset: $\ln \left(Z^{\text {neq }}(t) / Z^{\text {st }}\right)$ mostrando que asintóticamente $\ln Z^{\text {neq }}(t) \sim t^{-1}$.

desnudos.

Finalmente, consideraremos la dinámica de la discontinuidad en la distribución de momentos para $k=k_{F}$. Para tiempos cortos $v_{F} t \ll q_{c}^{-1}$ encontramos un decaimiento gaussiano de $Z^{\text {neq }}(t)$ desde su valor inicial de uno:

$$
Z^{\mathrm{neq}}(t)=\exp \left[-t^{2} \frac{4 f_{0}^{2} k_{F}}{(2 \pi)^{4}} c \int_{0}^{\infty} d q(F(q) q)^{2}+\mathscr{O}\left(f_{0}^{3}\right)\right],
$$

en donde $c$ es una constante de $\mathscr{O}(1)$ que viene de la integración angular sobre la superficie de Fermi. El decaimiento gaussiano a tiempos cortos es independiente de la forma específica de la interacción y también ocurre en $D=1[30,67,101]$. Para $v_{F} t \gg q_{c}^{-1}$, es necesario definir una forma explícita para la interacción. Para una forma simple, $f(q)=f_{0}\left(q / q_{c}\right)^{n} e^{-q / q_{c}}$, encontramos:

$$
Z^{\mathrm{neq}}(t) \approx Z^{\mathrm{st}} \exp \left[g^{2} a_{n}\left(v_{F} q_{c} t\right)^{-(2 n+1)}\right]
$$

en donde $Z^{\text {st }} \simeq\left(Z^{\text {eq }}\right)^{2}$ es el residuo de cuasipartícula en el estado estacionario, $a_{n}$ es una constante positiva adimensional que surge de las integrales angulares y hemos definido la constante de interacción adimensional $g=\frac{f_{0} \sqrt{k_{F} q_{c}}}{2 \pi^{2} v_{F}}$. Nótese que al expandir el exponente en 
potencias de $f_{0}$ las exponenciales en las Ecs. (3.82) y (3.83) se recupera el mismo comportamiento asintóticos de $Z^{\text {neq }}(t)$ que fue obtenido por medio del análisis perturbativo. En la Fig. 3.4 ilustramos la dinámica del residuo de cuasipartícula para diferentes intensidades de la interacción y $n=0$.

La imagen que surge del tratamiento del presente problema mediante bosonización sugiere que la primera etapa después del quench puede considerarse como la etapa en que los fermiones desnudos se «visten» debido a la influencia de la interacción en condiciones de no equilibrio (en contraste con el vestido adiabático de la teoría de Landau) para dar lugar a las cuasipartículas. Luego de que las cuasipartículas se forman (lo cual está señalado por el hecho de que $Z^{\text {neq }}(t)$ alcanza un valor estacionario no nulo) éstas empizan a colisionar entre ellas, posibilitando la relajación final hacia un estado térmico (el cual está caracterizado por $Z^{\text {neq }}=0$ ). Esta última etapa, por supuesto, no puede ser capturada por la bosonización. Como se ha visto en el capítulo anterior, un comportameinto similar de $Z^{\text {neq }}(t)$ puede observarse en sistemas en $D=1[30,67,101,74,120]$, la gran diferencia es que el residuo de cuasipartícula en el estado estacionario de no equilibrio es $Z_{1 D}^{\text {neq }}(t \rightarrow+\infty)=Z^{\text {st }}=0$, indicando que el vestido de no equilibrio lleva a una completa destrucción de las cuasipartículas fermiónicas en el sistema.

Para reforzar la imagen global, en la Fig. 3.5 mostramos los resultados para el $Z^{\text {neq }}(t)$ en el modelo de Hubbard en dimensión infinita obtenidos utilizando Teoría de Campo Medio Dinámica (DMFT por sus siglas en inglés) [40]. En el gráfico se puede ver claramente que, para interacciones lo suficientemente débiles (para las cuales se aplica nuestro análisis) $Z^{\text {neq }}(t)$ alcanza un plateau, compatible con el estado pretermalizado analizado en este capítulo, para luego relajar finalmente hacia su valor térmico, $Z^{\text {neq }}(t)=0$.

\subsection{Otras perspectivas teóricas sobre la pretermalización y eviden- cias experimentales}

Nuestra visión sobre la pretermalización, que se desprende del estudio que hemos expuesto en este capítulo y en [104], consiste en que para quenches débiles, en los que se inyecta poca energía al sistema, la dinámica a tiempos cortos puede ser descripta mediante un modelo efectivo bilineal. Esa afirmación implica que existe la posibilidad de que surga un estado metaestable a tiempos cortos y que ese estado metaestable esté descripto por un GGE que tome en cuenta las cantidades conservadas del modelo cuadrático (los números de ocupación de los modos libres). La relación entre dephasing, GGE y pretermalización es muy clara en este esquema una vez que el modelo efectivo cuadrático de tiempos cortos es identificado.

Existe un punto de vista complementario sobre la pretermalización que ha sido desarrollado por Kollar, Wolf y colaboradores [78]. Para describirlo, consideremos un modelo integrable cuyo Hamiltoniano puede escribirse como una suma pesada de sus cantidades conservadas $I_{\alpha}$, $H_{0}=\sum_{\alpha} \epsilon_{\alpha} I_{\alpha}$, en donde $\epsilon_{\alpha}$ es una especie de relación de dispersión generalizada. Supongamos 


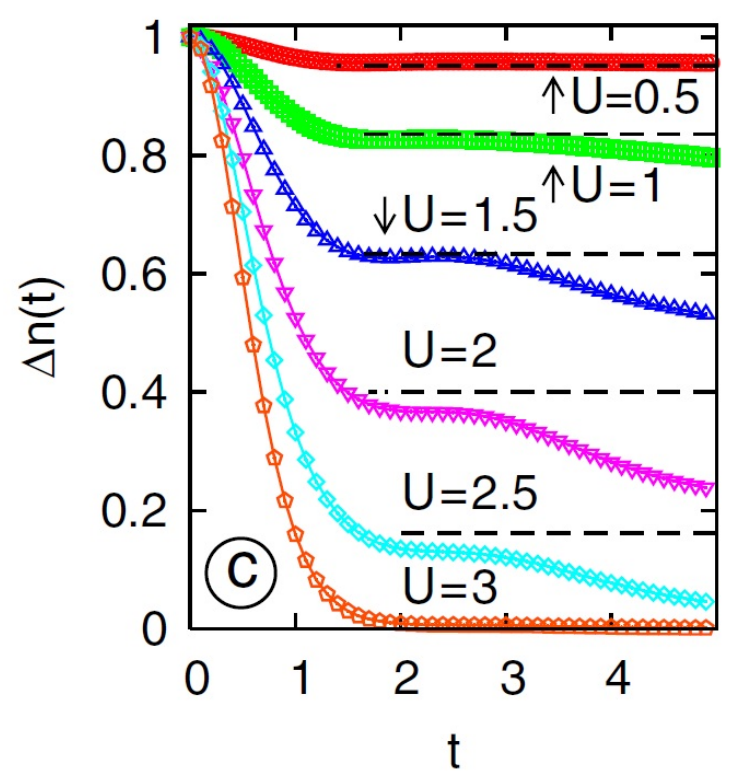

Figura 3.5: Pretermalización en el modelo de Hubbard fermiónico en dimensión infinita luego de un quench de interacción [40] obtenido mediante técnicas de DMFT. El residuo de cuasipartícula (llamado $\Delta n(t)$ en [40], $Z(t)$ para nosotros) muestra plateaus de pretermalización que son más pronunciados para interacciones débiles y tienden a desaparecer a medida que se aumenta el acoplamiento. Las líneas discontinuas horizontales corresponden a $2 Z^{\mathrm{eq}}-1$, demostrando la validez del resultado perturbativo discutido en la sección 3.1. Tomado de [40].

que preparamos al sistema en el estado fundamental de $H_{0}$ y que lo hacemos evolucionar mediante un Hamiltoniano que consiste del modelo integrable más un pequeño término que rompe la integrabilidad $H=H_{0}+g H_{1}$, con $g \ll 1$. El enfoque de los autores de [78] consiste en construir un conjunto de cantidades aproximadamente conservadas $\tilde{I}_{\alpha}$ perturbando las cantidades conservadas del modelo original $I_{\alpha}$. Estas cantidades están aproximadamente conservadas en el sentido de que conmutan entre ellas y con el Hamiltoniano de evolución al orden más bajo en teoría de perturbaciones,

$$
\begin{aligned}
{\left[H, \tilde{I}_{\alpha}\right] } & =\mathscr{O}\left(g^{2}\right), \\
{\left[\tilde{I}_{\alpha}, \tilde{I}_{\alpha^{\prime}}\right] } & =\mathscr{O}\left(g^{2}\right) .
\end{aligned}
$$

En [78] fue mostrado que el GGE construido con estas cantidades perturbadas predice el valor de los plateaus de pretermalización en un modelo débilmente interactuante de fermiones. En tal caso $H_{0}=\sum_{k} \epsilon(k) n(k)$ es un modelo de fermiones libres y las $I_{\alpha}$ son los números de ocupación $n(k)$ y, por lo tanto, su resultado guarda muchas similitudes con lo expuesto en este capítulo. El hecho de que esta construcción resulte válida para modelos integrables más complejos (por ejemplo, aquellos solubles por Bethe ansatz), en los que las cantidades conservadas ya no son simples números de ocupación, es un problema abierto a nuestro criterio. 


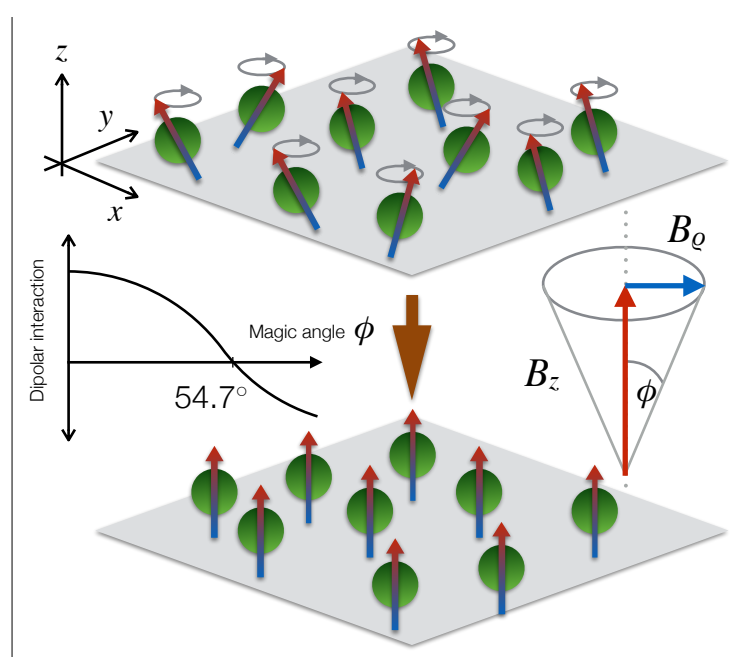

Figura 3.6: Protocolo para realizar un quench de interacciones isotrópicas en un gas de fermiones sin espín con interacciones dipolares. Ver texto para más explicaciones.

Estos dos puntos de vista sobre la pretermalización coinciden en que para quenches débiles en sistemas débilmente interactuantes, ésta se encuentra asociada a la existencia de ciertas cantidades que están aproximadamente conservadas a tiempos cortos y en que el estado estacionario que eventualmente emerge en la evolución puede describirse a través del GGE construido con ellas. Sin embargo, la existencia de cantidades conservadas efectivas a tiempos cortos no garantiza que existan estados metaestables, ni tampoco nos dice nada sobre su vida media, es decir, sobre las escalas de tiempo de la termalización. En el Capítulo 5 analizaremos la vida media de los estados pretermalizados lo que nos posibilitará distinguir cuáles son las variables relevantes que determinan la aparición de estos estados y su extensión temporal.

Mencionamos también que la emergencia de estados metaestables no térmicos de tiempos cortos ha sido observada en un experimento con condensados de Bose-Einstein 56]. En este experimento, se constató la naturaleza no térmica del estado estacionario al observar que la temperatura efectiva de tal estado era mucho menor que la temperatura del estado inicial. En un experimento más reciente [84], con una configuración experimental muy parecida al anterior, se ha demostrado que las funciones de correlación en tal estado estacionario pueden describirse con un GGE en ciertos modos cuasilibres fonónicos que son excitados por el quench. En ambos casos, el modelo teórico propuesto para explicar los resultados consiste en una collección de modos cuasilibres que relajan por dephasing, en completo acuerdo con la perspectiva presentada en esta tesis.

Finalmente, notamos que el quench de interacción en un gas de fermiones sin espín, estudiado en el presente capítulo, puede ser realizado experimentalmente en un gas de Fermi con interacciones dipolares, por ejemplo, átomos de ${ }^{167} \mathrm{Er}$ confinados en una trampa $2 D$, para los cuales se ha alcanzado el régimen degenerado recientemente [87]. Un posible protocolo para realizar el encendido repentino de interacciones está ilustrado en la Fig. 3.6. Debemos situar 
al gas de átomos dentro de un campo magnético constante $B_{z}$, perpendicular al plano del movimiento de los átomos junto con un campo magnético en rotación (producido por una corriente alterna) $B_{\rho}(t)$, el cual debe ser encendido adiabáticamente para preparar el estado inicial del sistema. Recordemos que el potencial de interacción entre dos dipolos eléctricos en $D=2$ tiene la forma:

$$
V_{d}(\mathbf{r})=\frac{d^{2}}{r^{3}}\left(1-3 \cos ^{2}(\theta)\right),
$$

en donde $d$ es el momento dipolar de los átomos, $r$ es la distancia entre ellos y $\theta$ es el ángulo entre el momento dipolar de los átomos y el eje perpendicular al plano de movimiento. Existe entonces el llamado ángulo mágico $\phi$, tal que $\cos ^{2}(\phi)=1 / 3$, para el cual las interacciones dipolares se anulan. Para preparar la condición inicial (gas sin interacciones), debemos entonces fijar el valor máximo de $B_{\rho} / B_{z}$ de manera que los momentos dipolares de los átomos realicen un movimiento de precesión alrededor del eje $z$ con un ángulo igual a $\phi$, para el cual las interacciones entre los átomos se promedian a cero (panel superior de la Fig. 3.6). A $t=0$ debe desconectarse el campo $B_{\rho}(t)$ lo que deja a los átomos interactuando con una interacción isotrópica de largo alcance correspondiente a $\theta=0$ (panel inferior de la Fig. 3.6), con lo cual se realizaría el quench de interacciones isotrópicas estudiado en este capítulo. Basándonos en los resultados expuestos en este capítulo, esperamos que para un quench débil el sistema exhibirá un estado pretermalizado. Éste se manifestará como un estado cuasiestacionario en un proceso de relajación en dos etapas: luego de un cambio inicial rápido, la distribución de momentos se establecerá en un estado diferente a una distribución térmica final. Sin embargo, la energía cinética debería alcanzar rápidamente su valor final. 



\section{Proyección dinámica y ecuaciones de movimiento fuera de equilibrio}

Si comparamos las figuras 3.4 y 3.5 podemos observar que la técnica de la bosonziación captura muy bien la existencia de los estados pretermalizados y, como se ha visto en el capítulo anterior, varias de sus propiedades. Sin embargo, la Fig. 3.5 muestra que los estados pretermalizados tienen una vida media finita, algo que no es posible capturar con bosonización, ya que los procesos que hacen inestables a los estados pretermalizados son los mismos que fueron truncados del Hamiltoniano para poder implementar la bosonización. En el transcurso de la investigación de la cual es fruto esta tesis nos encontramos entonces con la pregunta ¿cómo acceder a escalas de tiempo más largas que las que pueden ser capturadas mediante métodos como la bosonización? Los métodos numéricos usuales están confinados a tiempos cortos y sistemas grandes o a sistemas pequeños y tiempos arbitrarios, como por ejemplo t-DMRG o diagonalización exacta. La respuesta no podría estar en utilizar estos métodos. Por el lado analítico, el formalismo de Keldysh permite implementar toda la tecnología de los diagramas de Feynman al estudio de sistemas fuera de equilibrio. Sin embargo, un tratamiento perturbativo simple del problema de un quench de interacción provee información sólo sobre la evolución a tiempos cortos [97] ya que, en general, la expansión perturbativa genera términos seculares (que divergen con el tiempo). Para acceder a tiempos más largos es necesario usar complejas estrategias para resumar conjuntos de diagramas [6]. Decidimos entonces explorar otras opciones. El fruto de esta exploración es el material presentado en este capítulo.

Entre las diferentes alternativas teóricas a disposición para resolver la dinámica de no equilibrio de sistemas cuánticos aislados, el uso de diferentes tipos de ecuaciones de evolución para funciones de correlación de pocos tiempos está siendo explotado en la literatura contemporánea (ver, por ejemplo [136, 46, 135, 6]). En contraste con los métodos numéricos, la solución de ecuaciones de evolución permite investigar la dinámica de sistemas grandes para tiempos relativamente largos y usualmente demanda un esfuerzo numérico modesto. Sin embargo, la deducción de este tipo de ecuaciones comenzando de primeros principios, es decir, de la ecuación de la ecuación de Schrödinger o sus equivalentes, frecuentemente involucra una serie de aproximaciones no controladas [6, 7] que, aunque se utilizan rutinariamente, no son 
fáciles de justificar en todos los casos.

Por otro lado, el tipo más estudiado de ecuaciones de evolución son las ecuaciones cinéticas, que son ecuaciones markovianas -es decir, sin memoria- que aproximan la dinámica de funciones de correlación de una partícula. El ejemplo más notorio de este tipo de ecuaciones es la ecuación de Boltzmann. A pesar de que el límite markoviano está bien definido en algunas situaciones [54, 119] puede que no esté bien justificado en todos los casos. En particular, en situaciones en las que el sistema es reacio a borar información sobre las condiciones iniciales (como en modelos cerca de puntos integrables) las ecuaciones cinéticas pueden fallar en la descripción de la dinámica.

En este capítulo presentamos un método para construir ecuaciones de evolución para funciones de correlación de pocos puntos en sistemas débilmente interactuantes sin ninguna aproximación más allá de una serie perturbativa en el parámetro que mide la intensidad de las interacciones. Las ecuaciones resultantes son no markovianas. La aplicación de estas ecuaciones nos permitirá, en el capítulo siguiente, investigar la dinámica de tiempos largos de sistemas interactuantes y acceder al régimen que está más allá de la escala de tiempos en la que se forma el estado pretermalizado. La tecnología de cálculo que discutiremos está basada en la técnica del operador proyección, un viejo conocido de la física de no equilibrio $[54,119,12,126]$. Esta técnica es la encarnación matemática de un principio casi intuitivo de la dinámica de un sistema aislado, según el cual, si partimos de las ecuaciones que gobiernan la dinámica microscópica del sistema aislado (sean las leyes de Newton o la ecuación de Schödinger) e integramos un cierto conjunto de grados de libertad «irrelevantes», obtendremos una dinámica efectiva para los grados de libertad «relevantes» en la cual la influencia de los grados de libertad integrados se expresará como «disipación» y «ruido». En este sentido la diferencia entre un sistema autónomo y un sistema abierto no es fundamental: si nos concentramos sólo en ciertos grados de libertad el sistema autónomo parece como si fuera un sistema abierto y, a la inversa, el sistema abierto más su entorno conforman un sistema autónomo. En esta concepción, el espacio que queda para la creatividad (y por lo tanto para resolver problemas nuevos) es a qué llamamos grados de libertad relevantes y grados de libertad irrelevantes, o, en otras palabras, qué designamos como nuestro sistema y su entorno. En el caso de un sistema acoplado con un baño térmico la elección es clara, el sistema es el sistema y el baño térmico es el entorno o, en otras palabras, las variables del sistema son los grados de libertad relevantes y las del baño térmico las irrelevantes. En el caso de un sistema cuántico aislado podemos poner la frontera sistema-entorno en diferentes lugares. Podemos considerar, por ejemplo, a nuestro sistema como una parte acotada en el espacio físico del sistema total y al entorno como su complemento. Otra elección menos evidente, pero a menudo muy útil, es considerar a los grados de libertad de baja energía como los grados de libertad relevantes y a los de alta energía como los irrelevantes, algo en lo que hemos estado trabajando en el capítulo anterior ${ }^{1}$. En equilibrio, tal elección está en la base del grupo de renormalización. Otra elección, por lejos más abstracta, es considerar como variables relevantes

\footnotetext{
${ }^{1}$ En el capítulo anteior consideramos la relajación de un sistema en la etapa en la cual la dinámica de los grados de libertad de baja energía está completamente desacoplada de los grados de libertad de alta energía.
} 
a las funciones de correlación de baja complejidad (funciones de correlación de pocos puntos) iy a las funciones de correlación de alta complejidad como el entorno. Este enfoque está en la base de la llamada jerarquía BBGKY (Bogoliubov-Born-Green-Kirkwood-Yvon) utilizada para deducir la ecuación de Boltzmann clásica y, de hecho, está relacionado con la técnica que describiremos en este capítulo.

El ejemplo mejor estudiado de este principio de carácter universal es el movimiento browniano, el que pasaremos a discutir de una manera muy superficial y sólo atendiendo a nuestros objetivos más inmediatos (puede encontrarse discusiones completas en [81, 19]). Si consideramos la dinámica de una partícula A en interacción con un gran número de partículas de tipo $\mathrm{B}$ (mucho menos masivas que la partícula A), para resolver la dinámica el enfoque microscópico nos requerirá resolver las ecuaciones de Newton para la partícula A y todas las partículas B. Sin embargo, si sólo estamos interesados en la trayectoria de la partícula A (nuestros grados de libertad relevantes) podemos integrar los grados de libertad irrelevantes (las trayectorias de las partículas B) y obtener una dinámica efectiva para la partícula B. En particular, si llevamos a cabo la integración de los grados de libertad irrelevantes (o proyección) mediante alguna técnica particular (como por ejemplo la que discutiremos más adelante en este capítulo) obtendremos una ecuación de movimiento efectiva para la trayectoria $\mathbf{u}(t)$ de la partícula $\mathrm{A}$ que tendrá la forma:

$$
\frac{d \mathbf{u}(t)}{d t}=-\int_{-\infty}^{t} \gamma\left(t-t^{\prime}\right) \mathbf{u}\left(t^{\prime}\right) d t^{\prime}+\frac{1}{m} \mathbf{R}(t)
$$

en donde, sin entrar en muchos detalles, $\gamma(s)$ es el término de fricción o disipación, $\mathbf{R}(t)$ es el ruido y $m$ es la masa de la partícula A. Lo que habremos hecho entonces es cambiar la dinámica microscópica de la partícula A y todas las partículas B, la cual en general está determinada por ciertas ecuaciones diferenciales que son locales en el tiempo, por una dinámica efectiva para la trayectoria $\mathbf{u}(t)$ de la partícula A que es no local en el tiempo, ya que involucra la historia pasada de la partícula a través del término de memoria o de acción retardada (el primer término del lado derecho en la Ec. (4.1)). Por supuesto, las funciones $\gamma(s)$ y $\mathbf{R}(t)$ tendrán una expresión exacta en función de las variables microscópicas del sistema y contendrán la información acerca de las partículas B y la Ec. (4.1) será, en principio, tan difícil de resolver como la dinámica microscópica. Sin embargo, la Ec. 4.1 es un buen punto de partida para las aproximaciones. La aproximación más tradicional es convertir la Ec. (4.1) en una ecuación estocástica. Por ejemplo, si suponemos que el término de fricción es tal que $\gamma\left(t-t^{\prime}\right)=\gamma^{2}$ y $\mathbf{R}(t)$ un proceso estocástico gaussiano, la Ec. (4.1) se reduce a la conocida ecuación de Langevin que sirve para investigar tanto la dinámica de equilibrio como la de no equilibrio de la partícula browniana.

Los resultados originales de este capítulo fueron publicados en la Ref. [102], aunque en la presente exposición haremos un desarrollo mucho más detallado de los aspectos teóricos y

\footnotetext{
${ }^{2} \mathrm{Si}$ suponemos una partícula A esférica y aplicamos la ley de Stokes entonces $\gamma=6 \pi a \eta$ en donde $a$ es el radio de la esfera y $\eta$ es la viscosidad del fluido, es decir, una manera «macroscópica» de dar cuenta de la acción de todas las partíclas B.
} 
de aplicación de la técnica.

\subsection{Modelo y quench}

Por más que vayamos a hacer un tratamiento completamente general, es bueno tener en mente un problema más o menos acotado. Consideraremos un modelo general de fermiones sin espín interactuantes con Hamiltoniano $H=H_{0}+\alpha H_{1}$,

$$
H=H_{0}+\alpha H_{1}=\sum_{\mathbf{k}} \epsilon(\mathbf{k}) n(\mathbf{k})+\alpha \sum_{\mathbf{k}_{1}, \mathbf{k}_{2}, \mathbf{k}_{3}, \mathbf{k}_{4}} V_{\mathbf{k}_{3}, \mathbf{k}_{4}}^{\mathbf{k}_{1}, \mathbf{k}_{2}} c^{\dagger}\left(\mathbf{k}_{1}\right) c^{\dagger}\left(\mathbf{k}_{2}\right) c\left(\mathbf{k}_{3}\right) c\left(\mathbf{k}_{4}\right),
$$

en donde $c^{\dagger}(\mathbf{k})$ y $c(\mathbf{k})$ son operadores fermiónicos canónicos, $V_{\mathbf{k}_{3}, \mathbf{k}_{4}}^{\mathbf{k}_{1}, \mathbf{k}_{2}}$ es el elemento de matriz del potencial en el espacio de momentos, $\epsilon(\mathbf{k})$ es la relación de dispersión, $n(\mathbf{k})=c^{\dagger}(\mathbf{k}) c(\mathbf{k})$ es el operador número y $\alpha$ es una constante de acoplamiento adimensional. La hermiticidad del Hamiltoniano y la simetría en los índices de la suma en el Hamiltoniano de interacción $H_{1}$ imponen las siguientes condiciones $V_{\mathbf{k}_{3}, \mathbf{k}_{4}}^{\mathbf{k}_{1}, \mathbf{k}_{2}}=-V_{\mathbf{k}_{3}, \mathbf{k}_{4}}^{\mathbf{k}_{2}, \mathbf{k}_{1}}=-V_{\mathbf{k}_{4}, \mathbf{k}_{3}}^{\mathbf{k}_{1}, \mathbf{k}_{2}}=\bar{V}_{\mathbf{k}_{2}, \mathbf{k}_{1}}^{\mathbf{k}_{4}, \mathbf{k}_{3}}$, en donde $\bar{V}$ denota el complejo conjugado.

Estaremos interesados en el caso especial de un Hamiltoniano invariante traslacional según el cual las partículas interactúan a través de un potencial de a pares $v(\mathbf{x}-\mathbf{y})$. En tal caso

$$
V_{\mathbf{k}_{3}, \mathbf{k}_{4}}^{\mathbf{k}_{1}, \mathbf{k}_{2}}=\frac{1}{4 V} \delta_{\mathbf{k}_{1}+\mathbf{k}_{2}, \mathbf{k}_{3}+\mathbf{k}_{4}}\left(\hat{v}\left(\mathbf{k}_{1}-\mathbf{k}_{4}\right)-\hat{v}\left(\mathbf{k}_{2}-\mathbf{k}_{4}\right)-\hat{v}\left(\mathbf{k}_{1}-\mathbf{k}_{3}\right)+\hat{v}\left(\mathbf{k}_{2}-\mathbf{k}_{3}\right)\right),
$$

en donde $\hat{v}(\mathbf{k})=\int d \mathbf{r} v(\mathbf{r}) e^{i \mathbf{k} \cdot \mathbf{r}}$ es la transformada de Fourier del potencial y hemos escrito la forma antisimetrizada para respetar las condiciones de simetría del potencial. Estamos interesados en la evolución del sistema empezando desde una condición inicial de no equilibrio dada por una matriz densidad $\rho(0)$ invariante traslacional tal que $[H, \rho(0)] \neq 0$, pero que dejamos sin mayor especificación por el momento.

Dicho esto, aclaramos que la técnica que se detalla a continuación puede ser fácilmente aplicada a un sistema de bosones y que también es posible incluir spin.

\subsection{Técnica del operador proyección y ecuaciones de movimiento}

Para resolver la dinámica del sistema, nuestro punto de partida es la ecuación de Liouville en la representación de interacción $(\hbar=1)$ :

$$
\partial_{t} \tilde{\rho}(t)=-i \alpha\left[\tilde{H}_{1}(t), \tilde{\rho}(t)\right]=\alpha L(t) \tilde{\rho}(t),
$$

en donde $\tilde{O}(t)=e^{i H_{0} t} O e^{-i H_{0} t}$ es el operador $O$ en la representación de interacción y hemos introducido el superoperador de Liouville $L(t) O=-i\left[\tilde{H}_{1}(t), O\right]$. Nuestra tarea es econtrar soluciones aproximadas a la dinámica microscópica descripta por la ecuación de Liouville. La técnica del operador proyección (POT por sus siglas en inglés) define un programa para lograr 
esto. Primero debemos identificar las variables «lentas» o «macroscópicas» y luego proyectar la dinámica del sistema en el subespacio de esas variables lentas, el significado de lo cual se aclarará conforme procedamos. En un sistema invariante traslacional con interacciones débiles los números de ocupación emergen como las variables lentas naturales ${ }^{3}$ dado que $[H, n(\mathbf{k})]=\mathscr{O}(\alpha)$. Para llevar a cabo la proyección primero introduciremos la matriz densidad «relevante»

$$
\sigma(t)=\frac{1}{Z(t)} \exp \left[-\sum_{\mathbf{k}} \lambda(\mathbf{k}, t) n(\mathbf{k})\right]
$$

en deonde la función de partición dependiente del tiempo viene dada por

$$
Z(t)=\operatorname{Tr}\left[\exp \left(-\sum_{\mathbf{k}} \lambda(\mathbf{k}, t) n(\mathbf{k})\right)\right] .
$$

Nótese que $\tilde{\sigma}(t)=\sigma(t)$. Los multiplicadores de Lagrange $\lambda(\mathbf{k}, t)$ fuerzan la relación:

$$
\langle n(\mathbf{k})\rangle_{t} \equiv \operatorname{Tr}[n(\mathbf{k}) \sigma(t)]=\operatorname{Tr}[n(\mathbf{k}) \rho(t)] .
$$

En otras palabras, $\sigma(t)$ es la matriz densidad que maximiza la entropía de Von Neumann sujeta a las condiciones (4.7). La POT puede considerarse como la extensión de las ideas de Jaynes (discutidas brevemente en el Capítulo 1) a la dinámica fuera de equilibrio [126, 75].

Observamos que a pesar de que la dinámica de $\tilde{\rho}(t)$ es generada por la ecuación de Liouville, la matriz densidad relevante $\sigma(t)$ depende del tiempo independientemente de la representación. La proyección de la dinámica consiste en hallar una ecuación de movimiento para $\sigma(t)$. Para esto, introduciremos un superoperador de proyección $P(t)$ que proyecta la matriz densidad relevante $P(t) \tilde{\rho}(t)=\tilde{\sigma}(t)$ :

$$
P(t) \mu=\left(\sigma(t)-\sum_{\mathbf{k}} \frac{\delta \sigma(t)}{\delta\langle n(\mathbf{k})\rangle_{t}}\langle n(\mathbf{k})\rangle_{t}\right) \operatorname{Tr}[\mu]+\sum_{\mathbf{k}} \frac{\delta \sigma(t)}{\delta\langle n(\mathbf{k})\rangle_{t}} \operatorname{Tr}[n(\mathbf{k}) \mu],
$$

en donde $\mu$ es una matriz densidad arbitraria. El operador de proyección Ec. (4.8) está espe-

\footnotetext{
${ }^{3}$ En un sistema no homogéneo sería más conveniente elegir como variables lentas a los operadores $n(\mathbf{k}, \mathbf{q})=$ $c_{\mathbf{k}+\mathbf{q}}^{\dagger} c_{\mathbf{k}}$. La transformada de Fourier en $\mathbf{q}$ del valor de expectación de este operador es la función de Wigner de una partícula, la cual se utiliza para construir la descripción cinética de un sistema cuántico inhomogéneo. Con estos operadores se construye, por ejemplo, la teoría cinética de las cuasipartículas de Landau en un líquido de Fermi [5].
} 
cialmente diseñado para satisfacer las siguientes propiedades [54, 119, 12]:

$$
\begin{aligned}
P(t) \tilde{\rho}(t) & =\tilde{\sigma}(t), \\
P(t) \partial_{t} \tilde{\rho}(t) & =\partial_{t} \tilde{\sigma}(t), \\
\operatorname{Tr}[n(\mathbf{k}) P(t) \mu] & =\operatorname{Tr}[n(\mathbf{k}) \mu], \\
P(t) P\left(t^{\prime}\right) \mu & =P(t) \mu, \\
P(t) L(t) P(s) \mu & =0 .
\end{aligned}
$$

Para la prueba de las identidades pueden consultar el Apéndice A. La cuarta identidad, al poner $t=t^{\prime}$, expresa el carácter idempotente del proyector mientras que la última identidad depende de la forma explícita del Hamiltoniano $H$, en particular, del hecho de que el momento está conservado. También es útil definir el proyector complementario $Q(t)=1-P(t)$.

Siguiendo los pasos usuales [54, 119, 12, 126], partiendo de la ecuación de Liouville e introduciendo los proyectores obtenemos una ecuación para la dinámica de los grados de libertad lentos

$$
\partial_{t} P(t) \tilde{\rho}(t)=\alpha P(t) L(t) \tilde{\rho}(t)
$$

y otra para los grados de libertad rápidos, «microscópicos»

$$
\partial_{t} Q(t) \tilde{\rho}(t)=\alpha Q(t) L(t) \tilde{\rho}(t) .
$$

Insertando la identidad $I=P(t)+Q(t)$ en ambas ecuaciones obtenemos el sistema:

$$
\begin{aligned}
& \partial_{t} P(t) \tilde{\rho}(t)=\alpha P(t) L(t) P(t) \tilde{\rho}(t)+\alpha P(t) L(t) Q(t) \tilde{\rho}(t) \\
& \partial_{t} Q(t) \tilde{\rho}(t)=\alpha Q(t) L(t) P(t) \tilde{\rho}(t)+\alpha Q(t) L(t) Q(t) \tilde{\rho}(t)
\end{aligned}
$$

La ecuación de evolución para la matriz densidad relevante $\sigma(t)$ puede obtenerse resolviendo la ecuación para la parte irrelevante $Q(t) \tilde{\rho}(t)$ en la segunda línea del sistema e insertando la solución en la primer línea. La segunda línea es una ecuación diferencial lineal de primer orden inhomogénea en el operador $Q(t) \tilde{\rho}(t)$ (la inhomogeneidad es $\alpha Q(t) L(t) P(t) \tilde{\rho}(t)$ ) que puede ser resuelta (formalmente) de la misma manera que se resuelven ecuaciones diferenciales de funciones. La solución es:

$$
Q(t) \tilde{\rho}(t)=\alpha \int_{0}^{t} d s G(t, s) Q(s) L(s) P(s) \tilde{\rho}(s)+G(0, t) Q(0) \tilde{\rho}(0),
$$

en donde $G(t, s)$ es la exponencial ordenada (véanse los detalles en el Apéndice A)

$$
G(s, t)=\mathrm{T}_{\rightarrow} \exp \left[-\alpha \int_{s}^{t} d s^{\prime} Q\left(s^{\prime}\right) L\left(s^{\prime}\right)\right]
$$


es decir, $G(s, t)$ es la solución a la ecuación

$$
\begin{aligned}
\partial_{t} G(s, t) & =-\alpha G(s, t) Q(t) L(t), \\
G(s, s) & =I .
\end{aligned}
$$

Insertando (4.15) en la primera línea de (4.13) obtenemos la ecuación deseada:

$$
\begin{aligned}
\partial_{t} \tilde{\sigma}(t)=\alpha P(t) L(t) \tilde{\sigma}(t)+\alpha^{2} \int_{0}^{t} d s P(t) L(t) G(t, s) Q(s) L(s) \tilde{\sigma}(s) & \\
& +\alpha P(t) L(t) G(t, 0) Q(0) \tilde{\rho}(0) .
\end{aligned}
$$

El primer término en la Ec. (4.19) es un término tipo campo medio que se anula debido a la conservación del momento. El segundo puede ser completamente expresado en términos de la historia pasada de la distribución de momentos $\langle n(\mathbf{k})\rangle_{t}$, es decir, contiene memoria. Finalmente, el tercero es un ruido microscópico que no puede expresarse únicamente como función de las variables lentas. Es decir, hemos realizado rigurosamente el programa al que se aludía en la introducción, en la discusión alrededor de la ecuación de Langevin generalizada (4.1). Pero, ¿̇cuáles son los grados de libertad que hemos traceado?, o, en otras palabras, ¿qué representan nuestras variables irrelevantes? Dado que la matriz densidad relevante $\sigma(t)$ posee sólo la información acerca de las correlaciones de dos puntos (todas las funciones de correlación de dos puntos en un sistema homogéneo puede escribirse en términos de la distribución de momentos) los grados de libertad irrelevantes están relacionados con las correlaciones de más alto orden. Por otro lado, puede demostrarse con total generalidad (no hay necesidad de elegir una matriz densidad relevante explícitamente como en nuestro caso) [119] que la entropía de los grados de libertad relevantes $S_{r e l}(t)=\operatorname{Tr}[\sigma(t) \ln \sigma(t)]$ es tal que

$$
S_{\text {rel }}(t) \geq S_{\text {rel }}(0),
$$

es decir, cumple con la segunda ley de la termodinámica. Ahora podemos razonar de la siguiente manera: dado que, debido a la evolución puramente unitaria, la entropía del sistema aislado completo es constante $S(t)=\operatorname{Tr}[\rho(t) \ln \rho(t)]=$ cte y que la entropía de los grados de libertad relevantes en el estado final del sistema es mayor que en el estado inicial (en virtud de la propiedad (4.20)) el proceso de relajación o termalización del sistema puede comprenderse como un flujo de información -la información acerca de las condiciones iniciales- desde los grados de libertad relevantes hacia los irrelevantes. En nuestro caso, la información acerca de los detalles de la condición inicial fluirá desde las correlaciones de dos puntos hacia correlaciones de orden superior. Es decir, dado que es imposible que la información acerca de la condición inicial se pierda en un sistema con evolución unitaria ${ }^{4}$, el proceso de termalización de las funciones de correlación de pocos puntos puede entenderse como

\footnotetext{
${ }^{4}$ A nivel del estado cuántico no hay pérdida de información. Sin embargo la dinámica del los observables en general exhibe pérdida de información, por ejemploi, debido al dephasing.
} 
el flujo de esa información hacia las correlaciones de más alto orden. En otras palabras, la información detallada acerca de las condiciones iniciales no se borra en la evolución, sino que gradualmente se esconde en sectores poco accesibles del sistema.

Volviendo a los menesteres prácticos, notamos que afortunadamente el último término en la Ec. (4.19) (correspondiente al ruido microscópico) desaparece si elegimos una condición inicial que tenga la misma forma que la matriz densidad relevante, es decir, si $\rho(0)=\sigma(0)$, por lo que nos circunscribiremos a ese caso en lo que sigue. Este tipo decondiciones iniciales son matrices densidad gaussianas que representan estados descorrelacionados, tales como el estado fundamental de $H_{0}$ o un gas de fermiones libres a temperatura finita; lo cual nos lleva naturalmente a considerar un quench en la interacción.

La Ec. (4.19) es equivalente a la dinámica de Liouville y, en general, es tan difícil de resolver como el problema original. Sin embargo, este tratamiento es un buen punto de partida para realizar aproximaciones que no son evidentes en el lenguaje de la ecuación de Liouville. En el camino a resolver la Ec. (4.19) debemos realizar una expansión perturbativa en la constante adimensional de interacción $\alpha$ usando que $G(t, s)=I+\mathscr{O}(\alpha)$. Multiplicando por $n(\mathbf{k})$ y tomando la traza, utilizando que $\langle n(\mathbf{k})\rangle_{t}=\operatorname{Tr}[n(\mathbf{k}) \sigma(t)]$, obtenemos finalmente

$$
\partial_{t}\langle n(\mathbf{k})\rangle_{t}=\alpha^{2} \int_{0}^{t} d s \operatorname{Tr}[n(\mathbf{k}) L(t) L(s) \tilde{\sigma}(s)]+\mathscr{O}\left(\alpha^{3}\right) .
$$

Notamos entonces que surge una gran simplificación debido a la estructura gaussiana de $\sigma(t)$, lo que permite usar la regla de Wick para evaluar valores de expectación de cadenas de operadores de creación y aniquilación en (4.21). Después de un cálculo directo (pero potencialmente largo) obtenemos la siguiente ecuación de movimiento explícita

$$
\begin{aligned}
\partial_{t} f(\mathbf{k}, t) & =-16 \alpha^{2} \sum_{\mathbf{k}_{2}, \mathbf{k}_{3}, \mathbf{k}_{4}}\left|V_{\mathbf{k}_{3}, \mathbf{k}_{4}}^{\mathbf{k}, \mathbf{k}_{2}}\right|^{2} \int_{0}^{t} d s \cos \left[(t-s) \Delta e_{\mathbf{k}_{3}, \mathbf{k}_{4}}^{\mathbf{k}, \mathbf{k}_{2}}\right] \\
& \times\left(f(\mathbf{k}, s) f\left(\mathbf{k}_{2}, s\right) \bar{f}\left(\mathbf{k}_{3}, s\right) \bar{f}\left(\mathbf{k}_{4}, s\right)-f\left(\mathbf{k}_{3}, s\right) f\left(\mathbf{k}_{4}, s\right) \bar{f}(\mathbf{k}, s) \bar{f}\left(\mathbf{k}_{2}, s\right)\right)+\mathscr{O}\left(\alpha^{3}\right),
\end{aligned}
$$

en donde $\Delta e_{\mathbf{k}_{3}, \mathbf{k}_{4}}^{\mathbf{k}, \mathbf{k}_{2}}=\epsilon(\mathbf{k})+\epsilon\left(\mathbf{k}_{2}\right)-\epsilon\left(\mathbf{k}_{3}\right)-\epsilon\left(\mathbf{k}_{4}\right)$ es la variación de energía en una colisión de dos partículas y, para alivianar la notación, hemos definido $f(\mathbf{k}, t) \equiv\langle n(\mathbf{k})\rangle_{t} \mathrm{y} \bar{f}(\mathbf{k}, t) \equiv 1-\langle n(\mathbf{k})\rangle_{t}$. A continuación integramos en $t$ e intercambiamos los órdenes de integración [86]). Finalmente obtenemos una ecuación de Volterra (un sistema de ecuaciones en rigor):

$$
\begin{array}{r}
f(\mathbf{k}, t)=f(\mathbf{k}, 0)-16 \alpha^{2} \sum_{\mathbf{k}_{2}, \mathbf{k}_{3}, \mathbf{k}_{4}}\left|V_{\mathbf{k}_{3}, \mathbf{k}_{4}}^{\mathbf{k}, \mathbf{k}_{2}}\right|^{2} \int_{0}^{t} d s \frac{\sin \left[(t-s) \Delta e_{\mathbf{k}_{3}, \mathbf{k}_{4}}^{\mathbf{k}, \mathbf{k}_{2}}\right]}{\Delta e_{\mathbf{k}_{3}, \mathbf{k}_{4}}^{\mathbf{k}, \mathbf{k}_{2}}} \\
\times\left(f(\mathbf{k}, s) f\left(\mathbf{k}_{2}, s\right) \bar{f}\left(\mathbf{k}_{3}, s\right) \bar{f}\left(\mathbf{k}_{4}, s\right)-f\left(\mathbf{k}_{3}, s\right) f\left(\mathbf{k}_{4}, s\right) \bar{f}(\mathbf{k}, s) \bar{f}\left(\mathbf{k}_{2}, s\right)\right)+\mathscr{O}\left(\alpha^{3}\right),
\end{array}
$$

Esta ecuación ha aparecido en diferentes versiones varias veces en la literatura. En [ 119] fue 
derivada usando las mismas herramientas que presentamos aquí ${ }^{5}$ pero fue utilizada sólo como un paso intermedio para derivar la ecuación de Boltzmann. En las referencias [135] y [42] ${ }^{6}$ fue derivada usando argumentos heurísticos y utilizada para estudiar la dinámica de un sistema en dimensión infinita y para derivar (otra vez) la ecuación de Boltzmann, respectivamente. Queremos hacer hincapié en que la Ec. (4.23) es válida también para sistema en la red que sólo conservan cuasimomento (esto puede mostrarse sin mucho esfuerzo).

A pesar de que el método es claramente perturbativo, la solución a la Ec (4.23) va más allá de un tratamiento con teoría de perturbaciones usual porque la expansión perturbativa se realiza dentro de las ecuaciones integro-diferenciales y, por lo tanto, el acoplamiento está involucrado de una forma altamente no lineal. Evaluar la precisión de la aproximación no es una tarea fácil. Una posibilidad es calcular correcciones de orden mayor a Ec. (4.23), lo que puede realizarse sistemáticamente en el contexto de la técnica del operador proyección (esto convierte al desarrollo perturbativo utilizado para arribar a (4.23) en una aproximación controlada). Luego, el error podría ser calculado como la diferencia relativa entre la aproximación de más bajo orden y el orden siguiente [12]. No hemos realizado tal análisis formal del error pero nos hemos apoyado en otros criterios más intuitivos para confiar en los resultados de la Ec. (4.23). Por ejemplo, realizando una aproximación de tiempos cortos a (4.23) (los detalles pueden encontrarse en [135]) es posible rederivar resultados obtenidos por primera vez por Moeckel y Kehrein usando la técnica de la ecuación de flujo [95]. Además, en [135] se encontró que la solución de la Ec. (4.23) se compara bien con los resultados de DMFT para el modelo de Hubbard en dimensión infinita, en las escalas de tiempos accesibles. Por otro lado, en el Capítulo 2 hemos visto que la solución de esta ecuación describe bien la dinámica a tiempos cortos de un modelo unidimensional de fermiones, en particular, captura las leyes de potencias del modelo de Luttinger (un aspecto altamente no trivial de la dinámica), las cuales sabemos que están presentes en la dinámica del modelo gracias a resultados previos de DMRG [74]. Respecto a la validez de los resultados para escalas de tiempos más largas que las accesibles para las técnicas numéricas actuales, mencionamos que bajo ciertas suposiciones puede mostrarse [42] que la Ec. (4.23) se reduce, para tiempos lo suficientemente largos, a la ecuación de Boltzmann cuántica, la cual se espera que describa la termalización de sistemas aislados con interacciones débiles luego de que el sistema ha perdido memoria acerca de los detalles de la condición inicial [7].

Con respecto a la implementación de la Eq. (4.23), ésta puede ser resuelta usando técnicas estandard para sistemas de ecuaciones integrales de Volterra [ 86]. Un algoritmo directo que utilice, por ejemplo, la regla del trapecio para realizar la integral en el tiempo, implica un tiempo de cálculo que escalea como $L^{3 D} \times N^{2}$, en donde $N$ es el número de pasos temporales y $D$ la dimensión espacial del sistema. Hemos encontrado un algoritmo cuyo tiempo de ejecución escalea como $L^{3 D} \times N$ permitiéndonos alcanzar tiempos más largos y tamaños más grandes. Notamos que las ecuaciones de evolución se adaptan perfectamente al cálculo en

\footnotetext{
${ }^{5}$ Hemos encontrado esta referencia después de haber hecho la derivación de manera independiente. ¡A veces las cosas son así!

${ }^{6}$ Mucho más recientes que [119].
} 
paralelo.

Notemos que la Ec. (4.23) incluye colisiones cinéticas $\left(\Delta e_{\mathbf{k}_{3}, \mathbf{k}_{4}}^{\mathbf{k}, \mathbf{k}_{2}}=0\right)$ así como procesos no cinéticos. Para tiempos cortos, para los cuales las partículas aún no han colisionado, los procesos no cinéticos dominan la dinámica, mientras que para escalas de tiempo más largas las colisiones cinéticas dominan, y son las que conducen a la ecuación de Boltzman. Esto puede verse considerando el factor $\frac{\sin \left[(t-s) \Delta e_{\mathbf{k}_{3}, \mathbf{k}_{4}}^{\mathbf{k}, \mathbf{k}_{2}}\right]}{\Delta e_{\mathbf{k}_{3}, \mathbf{k}_{4}}}$ que aparece en la Ec. (4.23). Cuando $t-s$ es grande, el cociente se acerca a una $\delta\left(\Delta e_{\mathbf{k}_{3}, \mathbf{k}_{4}}^{\mathbf{k}, \mathbf{k}_{2}}\right)$, es decir, este factor fuerza la conservación de energía. Cuando $(t-s)$ es pequeño los procesos con un $\Delta e_{\mathbf{k}_{3}, \mathbf{k}_{4}}^{\mathbf{k}, \mathbf{k}_{2}}$ grande (procesos no cinéticos) tienen una probabilidad de ocurrencia apreciable. Una situación análoga surge al derivar de la regla de oro de Fermi utilizando teoría de perturbaciones dependiente del tiempo.

\subsection{Aproximaciones adicionales}

Las ecuaciones cinéticas (4.22) y (4.23) son muy ricas y complejas, lo que permite simplificarlas aun más para obtener otras aproximaciones a la dinámica que funcionan en diversas escalas de tiempo.

\subsubsection{Aproximación de tiempos cortos}

Dado que $\partial_{t} f(\mathbf{k}, t)=\mathscr{O}\left(\alpha^{2}\right)$, para pequeñas diferencias temporales podemos escribir

$$
f(\mathbf{k}, t)-f(\mathbf{k}, s)=\mathscr{O}\left(\alpha^{2}(t-s)\right) .
$$

Poniendo $t=0$ en la ecuación anterior y reemplazando en la Ec. (4.23) obtenemos una aproximación de tiempos cortos

$$
\begin{aligned}
f(\mathbf{k}, t)= & f(\mathbf{k}, 0)-8 \alpha^{2} \sum_{\mathbf{k}_{2}, \mathbf{k}_{3}, \mathbf{k}_{4}}\left|V_{\mathbf{k}_{3}, \mathbf{k}_{4}}^{\mathbf{k}, \mathbf{k}_{2}}\right|^{2} \frac{\sin ^{2}\left[\frac{t}{2} \Delta e_{\mathbf{k}_{3}, \mathbf{k}_{4}}^{\mathbf{k}, \mathbf{k}_{2}}\right]}{\left(\Delta e_{\mathbf{k}_{3}, \mathbf{k}_{4}}^{\mathbf{k}, \mathbf{k}_{2}}\right)^{2}} \\
& \times\left(f(\mathbf{k}, 0) f\left(\mathbf{k}_{2}, 0\right) \bar{f}\left(\mathbf{k}_{3}, 0\right) \bar{f}\left(\mathbf{k}_{4}, 0\right)-f\left(\mathbf{k}_{3}, 0\right) f\left(\mathbf{k}_{4}, 0\right) \bar{f}(\mathbf{k}, 0) \bar{f}\left(\mathbf{k}_{2}, 0\right)\right)+\text { Error, }
\end{aligned}
$$

en donde el error es del orden de máx $\left[\alpha^{3}, \alpha^{2} t\right]$. La aproximación Ec. (4.25) fue utilizada en el Capítulo 3 al hacer el análisis perturbativo fuera de equilibrio y constituye un resultado perturbativo al orden más bajo en el sentido usual.

\subsubsection{Ecuación de Boltzmann}

Es posible derivar la ecuación de Boltzmann cuántica realizando un escaleo en el tiempo en la Ec. (4.22). En el proceso de la derivación hay que hacer algunas suposiciones que no están completamente justificadas. Aquí nos reduciremos a describir el escaleo y dar el resultado, 
los interesados en la derivación pueden consultar las Refs. [42, 119]. El escaleo está diseñado para capturar la dinámica a tiempos largos y acoplamientos chicos:

$$
T=\alpha^{2} t
$$

Puede "mostrarse" entonces que en el límite $\alpha \rightarrow 0$ la ecuación cinética 4.22 se reduce a la ecuación de Boltzmann cuántica:

$$
\begin{array}{r}
\partial_{T} f(\mathbf{k}, T)=16 \pi \sum_{\mathbf{k}_{2}, \mathbf{k}_{3}, \mathbf{k}_{4}}\left|V_{\mathbf{k}_{3}, \mathbf{k}_{4}}^{\mathbf{k}, \mathbf{k}_{2}}\right|^{2} \delta\left(\Delta e_{\mathbf{k}_{3}, \mathbf{k}_{4}}^{\mathbf{k}, \mathbf{k}_{2}}\right) \\
\times\left(f(\mathbf{k}, T) f\left(\mathbf{k}_{2}, T\right) \bar{f}\left(\mathbf{k}_{3}, T\right) \bar{f}\left(\mathbf{k}_{4}, T\right)-f\left(\mathbf{k}_{3}, T\right) f\left(\mathbf{k}_{4}, T\right) \bar{f}(\mathbf{k}, T) \bar{f}\left(\mathbf{k}_{2}, T\right)\right) .
\end{array}
$$

La ecuación de Boltzmann Ec. (4.27) es local en el tiempo, es decir, desprecia todos los efectos de memoria expresados a través de integrales en el tiempo en las ecuaciones cinéticas más generales discutidas antes. Por lo tanto puede describir la dinámica del sistema en escalas de tiempo lo suficientemente grandes como para que el sistema haya perdido memoria de los detalles de la condición inicial [7]. Voveremos sobre este punto en el capítulo siguiente.

\subsubsection{Aproximaciones y mecanismos de relajación}

La aproximación de tiempos cortos captura la dinámica de dephasing a tiempos cortos y de hecho predice la presencia de plateaus de pretermalización en el modelo fermiónico estudiado en el Capítulo 3. La ecuación de Boltzmann, por otro lado, captura la dinámica producida por las colisiones entre partículas que domina a tiempos más largos. Por lo tanto, la Ec. (4.22) captura la dinámica del sistema en diferentes escalas de tiempo, aunando en una sola ecuación dos mecanismos de relajación muy diferentes: el dephasing, que conduce al estado pretermalizado y las colisiones entre partículas, que conduce al estado térmico final. Esto fue señalado por primera vez en [135].

\subsection{Funciones de correlación de orden más alto}

En esta sección extenderemos la técnica descripta anteriormente al caso de funciones de correlación de orden superior, en particular, a las correlaciones de dos tiempos de las variables lentas, relacionadas con las fluctuaciones. Para realizar la derivación debemos cambiar a la representación de Heisenberg, en la cual un operador sin dependencia temporal explícita evoluciona según la ley:

$$
\partial_{t} O=i[H, O] \equiv i L O
$$


Nótese la diferencia con el Liouvilliano definido en (4.4). La operación de traza define un operador proyección dual sobre el espacio de observables:

$$
\operatorname{Tr}[O P(t) \mu]=\operatorname{Tr}[\mu \mathrm{P}(t) O]
$$

en donde $O$ es un observable, $\mu$ una matriz densidad arbitraria y $\mathrm{P}(t)$ es el operador proyección en el espacio de observables. Su forma explícita puede obtenerse a partir de la ecuación anterior y de la Ec. (4.8):

$$
\mathrm{P}(t) O=\operatorname{Tr}[\sigma(t) O]+\sum_{\mathbf{k}}\left(n(\mathbf{k})-\langle n(\mathbf{k})\rangle_{t}\right) \operatorname{Tr}\left[\frac{\delta \sigma(t)}{\delta\langle n(\mathbf{k})\rangle_{t}} O\right] .
$$

Puede mostrarse que el proyector dual satisface las propiedades convenientes:

$$
\begin{aligned}
\mathrm{P}(t) \mathrm{P}\left(t^{\prime}\right) & =\mathrm{P}\left(t^{\prime}\right), \\
\dot{\mathrm{P}}(t) & =\mathrm{P}(t) \dot{\mathrm{P}}(t)(1-\mathrm{P}(t)) .
\end{aligned}
$$

También es útil definir el proyector complementario $\mathrm{Q}(t)=1-\mathrm{P}(t)$.

En la representación de Heisenberg la estrategia es separar las partes rápidas y lentas del superoperador de evolución mismo $e^{i L t}$ usando el proyector $\mathrm{P}(t)$ y su complemento $\mathrm{Q}(t)$ :

$$
e^{i L t}=e^{i L t} \mathrm{P}(t)+e^{i L t} \mathrm{Q}(t) .
$$

Usando la última identidad en la Ec. (4.31) obtenemos una ecuación para la parte irrelevante

$$
\partial_{t}\left[e^{i L t} \mathrm{Q}(t)\right]-e^{i L t} \mathrm{Q}(t) i L \mathrm{Q}(t)=e^{i L t} \mathrm{P}(t)[i L-\dot{\mathrm{P}}(t)] \mathrm{Q}(t),
$$

la cual puede ser resuelta formalmente usando el propagador $\overline{\mathrm{G}}(s, t)$ que satisface

$$
\begin{aligned}
\partial_{t} \overline{\mathrm{G}}(s, t) & =-i L \mathrm{Q}(t) \overline{\mathrm{G}}(s, t), \\
\overline{\mathrm{G}}(s, s) & =I,
\end{aligned}
$$

es decir, la exponencial ordenada temporalmente (detalles en el Apéndice A)

$$
\overline{\mathrm{G}}(s, t)=T_{\leftarrow} \exp \left[-\int_{s}^{t} d s^{\prime} i L \mathrm{Q}\left(s^{\prime}\right)\right] .
$$

La solución es

$$
e^{i L t} \mathrm{Q}(t)=e^{i L s} \mathrm{Q}(s) \overline{\mathrm{G}}(t, s)+\int_{s}^{t} d u e^{i L u} \mathrm{P}(u)[i L-\dot{\mathrm{P}}(u)] \mathrm{Q}(u) \overline{\mathrm{G}}(t, u) .
$$


Insertando la solución formal en la parte relevante obtenemos

$$
\begin{aligned}
e^{i L t}= & e^{i L t} \mathrm{P}(t)+\int_{s}^{t} d u e^{i L u} \mathrm{P}(u)(i L-\dot{\mathrm{P}}(u))(1-\mathrm{P}(u)) \overline{\mathrm{G}}(t, u) \\
& +e^{i L s}(1-\mathrm{P}(s)) \overline{\mathrm{G}}(t, s)
\end{aligned}
$$

en donde $s$ es un tiempo arbitrario $0 \leq s \leq t$. Para seguir la notación en la Ref. [54] definiremos una nueva exponencial ordenada (ver Apéndice A)

$$
\mathrm{G}(s, t)=T_{\leftarrow} \exp \left[\int_{s}^{t} d s^{\prime} i L \mathrm{Q}\left(s^{\prime}\right)\right] .
$$

Con esta definición podemos escribir finalmente la descomposición del superoperador evolución como

$$
\begin{aligned}
e^{i L t}= & e^{i L t} \mathrm{P}(t)+\int_{s}^{t} d u e^{i L u} \mathrm{P}(u)(i L-\dot{\mathrm{P}}(u))(1-\mathrm{P}(u)) \mathrm{G}(u, t) \\
& +e^{i L s}(1-\mathrm{P}(s)) \mathrm{G}(s, t) .
\end{aligned}
$$

Usando la forma explícita del superoperador proyección Ec. 4.30, es posible obtener una ecuación de operadores de tipo Langevin para las variables lentas [54],

$$
\begin{aligned}
\dot{n}(\mathbf{k}, t) \equiv e^{i L t} \dot{n}(\mathbf{k}) & =v_{\mathbf{k}}(t)+\sum_{\mathbf{k}^{\prime}} \Omega_{\mathbf{k}, \mathbf{k}^{\prime}}(t) \delta n(\mathbf{k}, t)+ \\
& +\int_{s}^{t} d u\left(K_{\mathbf{k}}(t, u)+\sum_{\mathbf{k}^{\prime}} \Phi_{\mathbf{k}, \mathbf{k}^{\prime}}(t, u) \delta n\left(\mathbf{k}^{\prime}, u\right)\right) \\
& +\eta_{\mathbf{k}}(t, s) .
\end{aligned}
$$

En donde hemos definido

$$
\begin{aligned}
\dot{n}(\mathbf{k}) & =i \operatorname{Ln}(\mathbf{k}), \\
\delta n(\mathbf{k}, t) & =n(\mathbf{k}, t)-\langle n(\mathbf{k})\rangle_{t},
\end{aligned}
$$

con $n(\mathbf{k}, t)=e^{i L t} n(\mathbf{k})$. El flujo organizado $\nu_{\mathbf{k}}(t)=\operatorname{Tr}\{n(\mathbf{k})[H, \sigma(t)]\}$ y las frecuencias colectivas $\Omega_{\mathbf{k}, \mathbf{k}^{\prime}}(t)=\operatorname{Tr}\left[\frac{\delta \sigma(t)}{\delta\langle n(\mathbf{k})\rangle_{t}} \dot{n}(\mathbf{k})\right]=\frac{\delta \nu_{\mathbf{k}}(t)}{\delta\langle n(\mathbf{k})\rangle_{t}}$, son cero debido a la conservación de momento en $H_{1}$. Las funciones de efecto retardado $K_{\mathbf{k}}(t, u)$ pueden expresarse en términos de los $\langle n(\mathbf{k})\rangle_{t}$ :

$$
K_{\mathbf{k}}(t, u)=\operatorname{Tr}[\sigma(u) i L Q(u) \mathrm{G}(u, t) \dot{n}(\mathbf{k})]=-\alpha^{2} \operatorname{Tr}\left\{\left[H_{1}(u),\left[H_{1}(t), n(\mathbf{k})\right]\right] \sigma(u)\right\}+\mathscr{O}\left(\alpha^{3}\right),
$$

y están relacionados con la dinámica de la distribución de momentos (véase la Ec. (4.21)). En la última igualdad hemos utilizado la última ecuación en (4.48) y $\mathrm{G}(u, t)=I+\mathscr{O}(\alpha)$. Las 
funciones de memoria $\Phi_{\mathbf{k}, \mathbf{k}^{\prime}}(u, t)$ son

$$
\begin{aligned}
\Phi_{\mathbf{k}, \mathbf{k}^{\prime}}(u, t)= & \operatorname{Tr}\left[\frac{\delta \sigma(u)}{\delta\left\langle n\left(\mathbf{k}^{\prime}\right)\right\rangle_{u}} i L \mathrm{Q}(u) \mathrm{G}(u, t) \dot{n}(\mathbf{k})\right] \\
& -\sum_{\boldsymbol{k}^{\prime \prime}}\left\langle n\left(\mathbf{k}^{\prime \prime}\right)\right\rangle_{u} \operatorname{Tr}\left[\frac{\delta^{2} \sigma(u)}{\delta\left\langle n\left(\mathbf{k}^{\prime}\right)\right\rangle_{u} \delta\left\langle n\left(\mathbf{k}^{\prime \prime}\right)\right\rangle_{u}} \mathrm{G}(u, t) \dot{n}(\mathbf{k})\right] .
\end{aligned}
$$

Puede mostrarse que estas funciones están relacionadas con las funciones de efecto retardado a través de una derivada funcional [54]

$$
\Phi_{\mathbf{k}, \mathbf{k}^{\prime}}(t, u)=\frac{\delta \int_{0}^{t} d s K_{\mathbf{k}}(t, s)}{\delta\left\langle n\left(\mathbf{k}^{\prime}\right)\right\rangle_{u}},
$$

lo cual constituye la relación clave entre la dinámica de la distribución de momentos y las fluctuaciones. Finalmente, hemos definido el ruido microscópico

$$
\eta_{\mathbf{k}}(t, s)=e^{i L s}(1-\mathrm{P}(s)) \mathrm{G}(s, t) \dot{n}(\mathbf{k}) .
$$

Si tomamos la traza con respecto a $\rho(0)$ de la Ec. (4.43) y nos quedamos con el orden más bajo en $\alpha$ recobraremos la ecuación cinética para la distribución de momentos Ec. (4.23). Pero nuestra intención no es rederivar esa ecuación sino hacer aproximaciones sobre la dinámica misma de los operadores para luego calcular funciones de correlación de orden más alto.

Poniendo $s=0$ en la ecuación de Langevin Ec. (4.43) y substrayendo el valor medio $\langle n(\mathbf{k})\rangle_{t}$ obtenemos una ecuación para las fluctuaciones del operador:

$$
\partial_{t} \delta n(\mathbf{k}, t)=\int_{0}^{t} d s \sum_{\mathbf{k}^{\prime}} \Phi_{\mathbf{k}, \mathbf{k}^{\prime}}(s, t) \delta n\left(\mathbf{k}^{\prime}, s\right)+\eta_{\mathbf{k}}(t)
$$

en donde $\delta n(\mathbf{k}, t)=e^{i L t} n(\mathbf{k})-\langle n(\mathbf{k})\rangle_{t} \mathrm{y}$

$$
\eta_{\mathbf{k}}(t)=\eta_{\mathbf{k}}(t, 0)-\operatorname{Tr}\left[\rho(0) \eta_{\mathbf{k}}(t, 0)\right]=\eta_{\mathbf{k}}(t, 0)
$$

A partir de esta definición del ruido es claro que $\left\langle\eta_{\mathbf{k}}(t)\right\rangle=\operatorname{Tr}\left[\rho(0) \eta_{\mathbf{k}}(t)\right]=0$.

Partiendo de la Ec. (4.53), usando la regla de Wick y evaluando explícitamente la derivada funcional en la Ec. (4.48) encontramos una ecuación de evolución explícita para la función de correlación temporal de las variables lentas $F_{\mathbf{k}, \mathbf{k}^{\prime}}(t) \equiv \operatorname{Tr}\left[\rho(0) \delta n(\mathbf{k}, t) \delta n\left(\mathbf{k}^{\prime}, 0\right)\right]$ :

$$
F_{\mathbf{k}, \mathbf{k}^{\prime}}(t)=F_{\mathbf{k}, \mathbf{k}^{\prime}}(0)-16 \alpha^{2} \int_{0}^{t} d s\left\{F_{\mathbf{k}, \mathbf{k}^{\prime}}(s) A_{\mathbf{k}}(t, s)+\sum_{\mathbf{q}} F_{\mathbf{q}, \mathbf{k}^{\prime}}(s)\left[B_{\mathbf{k}, \mathbf{q}}(t, s)-2 C_{\mathbf{k}, \mathbf{q}}(t, s)\right]\right\},
$$


en donde las matrices puedene escribise, usando la notación definida antes, como

$$
\begin{aligned}
& B_{\mathbf{k}, \mathbf{q}}(t, s)=\sum_{\mathbf{k}_{3}, \mathbf{k}_{4}}\left|V_{\mathbf{k}_{3}, \mathbf{k}_{4}}^{\mathbf{k}, \mathbf{q}}\right|^{2} \frac{\sin \left[(t-s) \Delta e_{\mathbf{k}_{3}, \mathbf{k}}^{\mathbf{k}, \mathbf{q}}\right]}{\Delta e_{\mathbf{k}}^{\mathbf{k}, \mathbf{q}}, \mathbf{k}_{4}}\left(f(\mathbf{k}, s) \bar{f}\left(\mathbf{k}_{3}, s\right) \bar{f}\left(\mathbf{k}_{4}, s\right)+\bar{f}(\mathbf{k}, s) f\left(\mathbf{k}_{3}, s\right) f\left(\mathbf{k}_{4}, s\right)\right), \\
& C_{\mathbf{k}, \mathbf{q}}(t, s)=\sum_{\mathbf{k}_{2}, \mathbf{k}_{4}}\left|V_{\mathbf{q}, \mathbf{k}_{4}}^{\mathbf{k}, \mathbf{k}_{4}}\right|^{2} \frac{\sin \left[(t-s) \Delta e_{\mathbf{q}, \mathbf{k}_{4}}^{\mathbf{k}_{2}, \mathbf{k}_{2}}\right]}{\Delta e_{\mathbf{q}, \mathbf{k}_{4}}^{\mathbf{k}, \mathbf{k}_{2}}}\left(f(\mathbf{k}, s) f\left(\mathbf{k}_{2}, s\right) \bar{f}\left(\mathbf{k}_{4}, s\right)+\bar{f}(\mathbf{k}, s) \bar{f}\left(\mathbf{k}_{2}, s\right) f\left(\mathbf{k}_{4}, s\right)\right),
\end{aligned}
$$

y $A_{\mathbf{k}}(t, s)=\sum_{\mathbf{k}^{\prime}} B_{\mathbf{k}^{\prime}, \mathbf{k}}(t, s)$. Para arribar a la Ec. (4.55) sólo hay que tener en cuenta que $\Phi_{\mathbf{k}, \mathbf{k}^{\prime}}(t, s)=$ $\mathscr{O}\left(\alpha^{2}\right)$ y que $\mathrm{G}(s, t)=I+\mathscr{O}(\alpha)$. Nótese que las matrices satisfacen la propiedad $A_{\mathbf{k}}(t, t)=$ $B_{\mathbf{k}, \mathbf{q}}(t, t)=C_{\mathbf{k}, \mathbf{q}}(t, t)=0$, la cual refleja causalidad, es decir que el valor de las fluctuaciones a tiempo $t$ sólo depende de la historia de las fluctuaciones a tiempos estrictamente anteriores a $t$. Una afirmación similar puede hacerse en el caso de la ecuación para la distribución de momentos, Ec. (4.23): el núcleo de la ecuación integral se anula para $s=t$. Esta propiedad de las ecuaciones de evolución trae aparejada una gran simplificación técnica dado que no es necesario resolver ecuaciones autoconsistentes a cada paso temporal de la integración numérica.

Hasta donde podemos saber, la Ec. (4.55) fue presentada por primera vez por nosotros en [102] y constituye uno de los aportes originales más importantes de esta tesis.

Para resolver la Ec. (4.55) necesitamos primero conocer la dinámica de la distribución de momentos, por ejemplo, resolviendo la Ec. (4.23), para poder determinar los coeficientes $A_{\mathbf{k}}(t, s), B_{\mathbf{k}, \mathbf{q}}(t, s)$ and $C_{\mathbf{k}, \mathbf{q}}(t, s)$. Conociendo la trayectoria de la distribución de momentos, la Ec. (4.55) se presta a la resolución numérica tanto como la Ec. (4.23). Finalmente, señalamos que dado que la técnica del operador proyección en la representación de heisenberg trabaja directamente sobre el operador de evolución mismo, sería posible obtener ecuaciones de evolución similares para otras funciones de correlación. 



\section{Estados pretermalizados y comporta- miento tipo vidrio en sistemas casi integrables en una dimensión}

En los capítulos anteriores, a través del análisis de diferentes modelos y discusiones generales, hemos tratado de delinear la estrecha relación que existe entre la pretermalización, el dephasing y el ensamble de Gibbs generalizado. En este capítulo abordaremos la pregunta lógica acerca de qué sucede después de la pretermalización, es decir, cuál es la vida media de los estados pretermalizados y cómo es el tránsito del sistema hacia el estado térmico final. Para lograr esto debemos acceder a la dinámica de tiempos largos de sistemas de muchos cuerpos, lo que constituye un desafío técnico importante. Utilizaremos dos técnicas completamente diferentes para lograr el objetivo: por un lado resolveremos las ecuaciones de movimiento deducidas en el capítulo anterior para un sistema de fermiones débilmente interactuantes y, por otro lado, utilizaremos una técnica semiclásica (la aproximación de Wigner truncada, TWA por sus siglas en inglés) para atacar un problema de bosones en todo el rango de interacciones para altas densidades. Estudiaremos ambos sistemas en las cercanías de un punto integrable, variando las condiciones iniciales y la distancia a la integrabilidad, lo que equivale a variar la disponibilidad de canales de relajación de la dinámica.

Si bien utilizamos técnicas muy diferentes para analizar estos dos modelos paradigmáticos de la física de fermiones y bosones en $D=1$, encontramos que emergen ciertas similitudes en el análisis de los resultados. Principlamente, hallamos que si nos encontramos lo suficientemente cerca de la integrabilidad (lo que restringe fuertemente los canales de relajación del sistema) la vida media de los estados metaestables (pretermalizados) que se observan puede llegar a ser de varios órdenes de magnitud, medidas en las escalas de tiempo naturales del sistema. En el caso de los fermiones, pudimos identificar que otro factor determinante de la vida media de los estados pretermalizados es la energía de la condición inicial, una variable que no fue investigada en el caso de los bosones. De todas maneras, la presencia de estados metaestables con una vida media tan alta puede obstaculizar completamente la observación del estado térmico final que el sistema debe alcanzar (tratándose de un sistema no integrable). En otras palabras, puede que los sistemas débilmente no integrables termalicen en el límite 


\section{Capítulo 5. Estados pretermalizados y comportamiento tipo vidrio en sistemas casi integrables en una dimensión}

termodinámico (recordemos que para sistemas finitos puede ser necesaria una ruptura finita de integrabilidad para termalizar, tal como fue discutido en el Capítulo 1), tal como indican los resultados recientes en [122], sin embargo es posible que, en ciertas condiciones, su proceso de termalización sea extremadamente largo y que el estado térmico sea virtualmente inobservable.

En el caso de los fermiones débilmente interactuantes también encontramos otro fenómeno interesante: variando el parámetro que rompe la integrabilidad existe un umbral bien definido más allá del cual, para los estados iniciales considerados, los estados metaestables desaparecen completamente. Es posible que este fenómeno no esté relacionado directamente con la rotura de la integrabilidad sino con una característica específica del modelo considerado: la apertura de un gap en el espectro del sistema interactuante, el cual modifica completamente las propiedades del sistema y su dinámica. Sin embargo, es interesante notar que este tipo de transición abrupta también fue encontrada en otros modelos, como el modelo de Hubbard en el límite de dimensión infinita $[40,130]$ y que, aunque los autores de esos artículos no lo analizaron en estos términos, pensamos que también en esos casos puede atribuirse a un cambio abrupto en el espectro del sistema, el cual está relacionado, posiblemente, con una transición de fase cuántica en equilibrio.

La aparición de estados pretermalizados con vidas medias tan grandes es muy similar, fenomenológicamente, a la física de los sistemas clásicos «vidriosos», tales como vidrios de spin [24], modelos atomísticos de vidrios [29, 32] y modelos con ligaduras cinéticas (KCM por sus siglas en inglés) [9]. Todos estos sistemas exhiben una relajación en dos etapas: después de un régimen inercial inicial (análogo al régimen de dephasing) se quedan atrapados en estados metaestables cuya vida media puede ser de escalas geológicas en ciertas regiones de parámetros, para temperaturas lo suficientemente bajas o densidades lo suficientemente altas en el caso de los modelos atómicos. Al analizar el problema de los bosones, llevaremos esta relación más allá de una analogía en la fenomenología al señalar que las trayectorias clásicas relacionadas con la dinámica cuántica exhiben uno de los sellos de la dinámica de los vidrios: la heterogeneidad dinámica.

Queremos señalar que la existencia de estados metaestables no térmicos con vidas medias muy largas puede tener relevancia experimental, ya que tales etados cuasiestacionarios de no equilibrio exhiben propiedades físicas muy diferentes de los estados estacionarios de equilibrio usualmente estudiados en el campo de la materia condensada.

En suma, en este capítulo presentamos los resultados obtenidos más recientemente, los que completan una especie de panorama general de la dinámica de no equilibrio después de un quench y, al mismo tiempo, dejan más interrogantes abiertos y abren nuevas perspectivas.

Las contribuciones originales de este capítulo se encuentran publicadas en las Refs. [83] $\mathrm{y}[103]$. 


\subsection{Fermiones débilmente interactuantes}

\subsubsection{Modelo}

Consideraremos primero un modelo de fermiones sin espín en una dimensión. El Hamiltoniano es $H\left(J_{1}, J_{2}, \Delta\right)=H_{0}\left(J_{1}, J_{2}\right)+H_{1}(\Delta)$ :

$$
\begin{aligned}
& H_{0}\left(J_{1}, J_{2}\right)=-J_{1} \sum_{j=0}^{L-1}\left(c_{j}^{\dagger} c_{j+1}+\text { h.c. }\right)-J_{2} \sum_{j=0}^{L-1}\left(c_{j}^{\dagger} c_{j+2}+\text { h.c. }\right), \\
& H_{1}(\Delta)=\Delta \sum_{j=0}^{L-1} n_{j} n_{j+1},
\end{aligned}
$$

en donde $J_{1}\left(J_{2}\right)$ es el hopping a primeros (segundos) vecinos, $\Delta$ es la intensidad de la interacción a primeros vecinos, $L$ es el número de sitios de la cadena, los operadores $c$ obedecen reglas de anticonmutación canónicas y $n_{j}=c_{j}^{\dagger} c_{j}$. Asumimos condiciones de contorno abiertas, $c_{L+m}^{\#}=c_{m}^{\#}$. Para $J_{2}=0$ el modelo puede mapearse al modelo $X X Z$ usando una transformación de Jordan Wigner, el cual puede resolverse exactamente (se pueden calcular todas las autofunciones y autoenergía del Hamiltoniano) a través del Bethe ansatz, mientras que para $J_{2} \neq 0$ el modelo es no integrable.

Como siempre, estamos interesados en la dinámica del sistema a partir de una condición inicial de no-equilibrio $\rho(0)$ tal que $[H, \rho(0)] \neq 0$. Para utilizar las ecuaciones de evolución deducidas en el capítulo anterior, nos restringiremos al caso débilmente interactuante $\alpha=$ $\Delta / J_{1} \ll 1$ y consideraremos sólo estados iniciales homogéneos. En tal caso, como se ha visto antes, los operadores que definen la distribución de densidad en el espacio de momentos

$$
\hat{n}_{k}=\frac{1}{L} \sum_{i, j} e^{-\frac{2 \pi k}{L}(i-j)} c_{i}^{\dagger} c_{j}
$$

con $k=0, \ldots, L-1$, emergen como las variables lentas naturales del sistema. Utilizando la técnica del operador proyección en el capítulo anterior fuimos capaces de deducir ecuaciones de evolución aproximadas para el valor medio de las variables lentas $n(k, t)=\left\langle\hat{n}_{k}(t)\right\rangle$, y las correlaciones de las fluctuaciones $F\left(k, k^{\prime}, t\right)=\left\langle\hat{n}_{k}(t) \hat{n}_{k^{\prime}}\right\rangle-\left\langle\hat{n}_{k}(t)\right\rangle\left\langle\hat{n}_{k^{\prime}}\right\rangle$, en donde $\langle\ldots\rangle=$ $\operatorname{Tr}[\rho(0) \ldots]$. Recordemos que las ecuaciones íntegro-diferenciales que se deducen con la técnica del operador proyección son tratables numéricamente si (i) nos restringimos a condicione iniciales no correlacionadas de la forma

$$
\rho(0)=\frac{1}{Z} e^{-\sum_{k} \lambda(k, 0) \hat{n}_{k}},
$$

con $Z=\operatorname{Tr}\left[e^{-\sum_{k} \lambda(k, 0) \hat{n}_{k}}\right]$, las cuales incluyen diferentes estados de fermiones libres, y (ii) nos quedamos sólo con el orden dominante en $\alpha$. Fijamos $\alpha=0,2$ por el resto de la exposición. 
Capítulo 5. Estados pretermalizados y comportamiento tipo vidrio en sistemas casi integrables en una dimensión
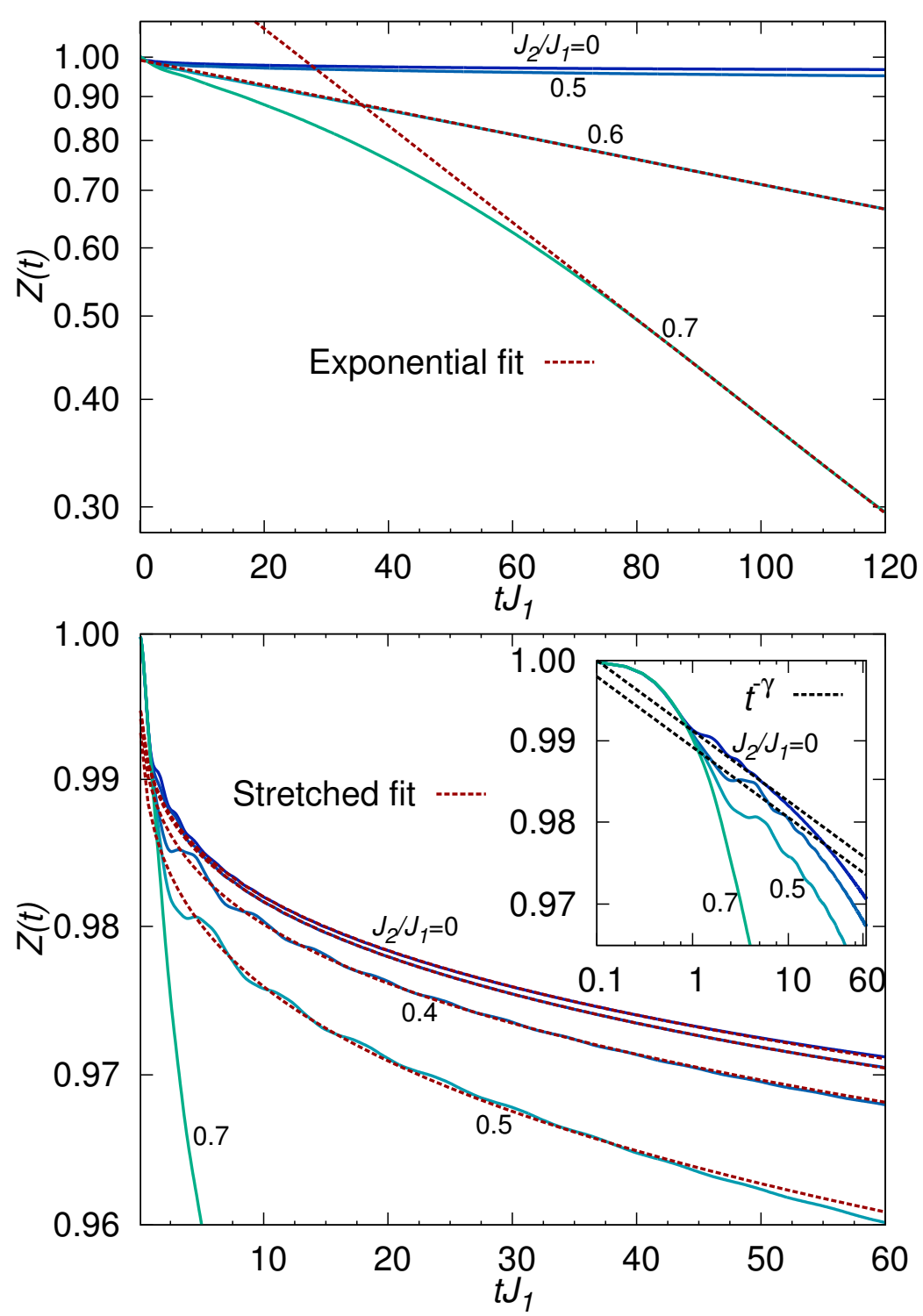

Figura 5.1: Decaimiento del residuo de cuasipartícula $Z(t)$ para diferentes valores de $J_{2}$ en un sistema de $L=1024$ sitios, y lejos de cualquier efecto de tamaño finito. Panel superior: Gráfico en escala semilogarítmica. Las líneas discontínuas (rojas) son ajustes exponenciales. Panel inferior: Gráfico semilogarítmico mostrando el decaimiento para $J_{2} \leq 0,5 J_{1}$; la curva para $J_{2}=0,7 J_{1}$ se presenta para comparación. Las líneas discontínuas (rojas) son ahora ajustes con exponenciales estiradas, dado que el ajuste con una exponencial no es posible. Inset: Gráfico Log-log. Las líneas discontínuas (negras) son leyes de potencia con el exponente dado por las predicciones del ML $Z(t) \sim t^{-\gamma}$, con $\gamma=\frac{1}{4}\left(K^{2}+K^{-2}-2\right)$. El parámetro de Luttinger $K$ se obtiene con bosonización, $K=\sqrt{\frac{\pi v_{F}-\Delta}{\pi v_{F}+3 \Delta}}$, en donde $v_{F}$ es la velocidad de Fermi. 


\subsubsection{Dinámica en el límite termodinámico}

Comenzamos considerando el efecto de variar la intensidad del parámetro de rotura de la integrabilidad $J_{2}$. Como estado inicial consideraremos el estado fundamental $\left|\Psi_{0}\right\rangle$ de $H_{0}\left(J_{1}, 0\right)$ con distribución de momentos $n(k, 0)=\theta\left(\epsilon_{0}(k)-\epsilon_{0}\left(k_{F}\right)\right)$, en donde $\epsilon_{0}(k)=-2 J_{1} \cos (2 k \pi / L)$. Trabajaremos a llenado medio, $k_{F}=L / 4$. Primero nos enfocaremos en la relajación de los modos de momento más cercanos a los puntos de Fermi. Estudiaremos, en particular, el decaimiento del residuo de cuasipartícula, definido como $Z(t)=n\left(k_{F}, t\right)-n\left(k_{F}+\frac{2 \pi}{L}, t\right)$. Tal como fue discutido en el Capítulo 3, en dimensiones superiores esta cantidad exhibe "plateaus de pretermalización" típicos, y se ha convertido en una herramienta estadar para detectar estados metaestables en sistemas fermiónicos [95, 40, 104]. En una dimensión esta cantidad no exhibe plateaus [60] (ver también el final del Capítulo 2). Más adelante veremos que, sin embargo, los estados metaestables pueden ser detectados usando otros observables. En el panel superior de la Figura 5.1 mostramos el decaimiento del residuo de cuasiártícula para diferentes valores de $J_{2}$ para un sistema con $L=1024$ y para tiempos que se encuentran lejos de cualquier efecto de recurrencia. En tal caso las correcciones de tamaño finito son despreciables y estamos accediendo por lo tanto a la dinámica del límite termodinámico. Es claramente visible que para $J_{2} \leq 0,5 J_{1}$ el decaimiento de $Z(t)$ es extremandamente lento (subexponencial) mientras que para $J_{2}>0,5 J_{1}$ exhibe una relajación exponencial. Con respecto a la evolución lenta encontramos que después de una evolución inicial de tipo gaussiana que sucede para $t J_{1}<1$, hay un decaimiento que es más rápido que una ley de potencias pero más lento que una exponencial. En ese régimen podemos ajustar una exponencial estirada $Z(t)=$ $e^{-(t / \tau)^{\beta}}$,en donde ambos $\tau$ y $\beta$ depende de $\Delta$ y $J_{2}$. En los ajustes mostrados en el panel inferior de la Figura 5.1, $\tau J_{1} \sim 10^{7}$ y $\beta \sim 0,27\left(J_{2}<0,5 J_{1}\right)$. Por otro lado, los decaimientos exponenciales que se dan para $J_{2}>0,5 J_{1}, \tau J_{1} \sim 10^{2}$. Más adelante veremos que tal incremento abrupto de las escalas de relajación (y el cambio de régimen de decaimiento exponencial a exponencial estirado) está relacionado con un incremento abrupto en la vida media de los estados metaestables. Tal relajación lenta de observables ha sido observada también en sistemas cuánticos abiertos [110]. Finalmente notamos que para $J_{2} \leq 0,5 J_{1}$, luego de la evolución gaussiana inicial, la tendencia inicial de las exponenciales estiradas queda muy bien descripta por la ley de potencias predichas por la dinámica de no equilibrio del modelo de Luttinger [30, 67], tal como fue discutido en el Capítulo 2. En el estudio de t-DMRG de la Ref. [74], en donde este hecho fue descubierto por primera vez, tal tendencia inicial era la única porción accesible de la dinámica. Esto demuestra la potencia del método desarrollado .

El cambio abrupto de comportamiento puede deberse a la apertura de una gap en el espectro del sistema interactuante. De hecho, para $J_{2}>0,5 J_{1}$ la relación de dispersión desnuda $\epsilon(k)$ de $H$ desarrolla dos puntos de Fermi adicionales, y por lo tanto el modelo de Luttinger que representa el comportamiento genérico de baja energía en equilibrio posee cuatro especies de fermiones. En este tipo de modelo una pequeña interacción entre los fermiones es relevante , y por lo tanto suficiente para la apertura de una gap en el espectro de excitaciones ${ }^{1}$. De

\footnotetext{
${ }^{1}$ Agradecemos a Volker Meden por llamarnos la atención sobre este punto
} 


\section{Capítulo 5. Estados pretermalizados y comportamiento tipo vidrio en sistemas casi integrables en una dimensión}

hecho, la apertura de un gap es una explicación probable al hecho de que para $J_{2}>0,5 J_{1}$ las predicciones del ML dejen de valer en la dinámica de $Z(t)$, que cambia a un decaimiento exponencial.

\section{Funciones de correlación de dos tiempos}

En una dimensión el salto de la distribución de momentos en el momento de Fermi no muestra ningún valor cuasiestacionario. De mostrarlo, sería una indicación de que existen cuasipartículas en el sistema que se están vistiendo y organizando dinámicamente a tiempos intermedios, y esto no es posible en una dimensión (al contrario de dimensión más alta, como se vio en capítulos anteiores), donde sólo las excitaciones colectivas están permitidas. Hemos encontrado que son entonces las funciones de correlación de dos tiempos las que resultan adecuadas para detectar y analizar los estados pretermalizados en nuestro sistema unidimensional. En particular, utilizaremos la función de correlación conectada del operador corriente total, $\hat{J}=\frac{1}{2 i} \sum_{j} c_{j}^{\dagger} c_{j+1}-c_{j+1}^{\dagger} c_{j}$ :

$$
C_{J}(t)=\langle\hat{J}(t) \hat{J}\rangle-\langle\hat{J}(t)\rangle\langle\hat{J}\rangle,
$$

la cual puede obtenerse a partir de las fluctuaciones de las variables lentas

$$
C_{J}(t)=\sum_{k, k^{\prime}} \sin (2 k \pi / L) \sin \left(2 k^{\prime} \pi / L\right) F\left(k, k^{\prime}, t\right),
$$

en donde recordamos que $F\left(k, k^{\prime}, t\right)=\left\langle\hat{n}_{k}(t) \hat{n}_{k^{\prime}}\right\rangle-\left\langle\hat{n}_{k}(t)\right\rangle\left\langle\hat{n}_{k^{\prime}}\right\rangle$. Las funciones de correlación conectadas de dos tiempos de observables locales son una herramienta estandar para diagnosticar la ergodicidad o no ergodicidad de la dinámica de equilibrio tanto en sistemas cuánticos [72, 117, 118] como clásicos [29]. Se espera que este tipo de correladores decaiga rápidamente a cero para sistemas ergódicos dado que el estado inicial y el estado a tiempo $t$ deben encontrarse completamente descorrelacionados más allá de una cierta escala de tiempos característica, es decir, se espera que el sistema pierda memoria de las condiciones iniciales. Una saturación de este correlador a un valor constante no nulo señala por lo tanto la pérdida de ergodicidad. Un comportamiento intermedio, con un plateau que emerge entre la evolución incial rápida y el decaimiento final a cero ocurre en sistemas que quedan atrapados por largo tiempo en estados metaestables. El ejemplo más prominente de este comportamiento son los sistemas vidriosos, tales como los vídrios de espín y los líquidos sobreenfriados [24, 29]. Ilustramos esto en la Fig. 5.2, en la que mostramos el decaimiento de la función intermedia de scattering incoherente $F_{s}(q, t)$ (la función de correlación de las fluctuaciones de la densidad a un dado momento $q$, que mide el solapamiento de configuraciones a tiempo $t$ y en el instante inicial), que es una función de correlación de dos tiempos análoga a $C_{J}(t)$. En el gráfico se muestra el decaimiento de $F_{s}(q, t)$ para un fluído clásico con interacciones de Lennard-Jones en equilibrio. Si bien es un caso completamente diferente al nuestro, ya que es un sistema clásico, con una condición inicial de equilibrio y con una dinámica que posee elementos estocásticos para simular un baño térmico, veremos que nuestro sistema 


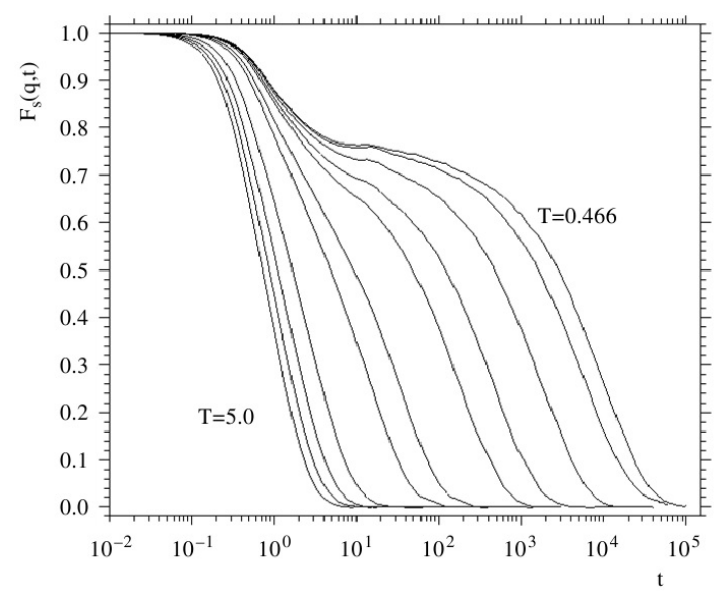

Figura 5.2: Decaimiento de la función de correlación de las fluctuaciones de densidad $F_{s}(q, t)$ para un fluido de Lennard-Jones clásico en equilibrio para diferentes temperaturas $T$. Al bajar la temperatura por debajo de un cierto umbral se observa la emergencia de estados estacionarios cuyas vidas medias son muy grandes para temperaturas suficientemente bajas. Nótese la escala logarítmica en el eje temporal. Tomado de [29].

presenta una física parecida: después de una evolución rápida inicial la dinámica se queda atrapada en estados metaestables cuyas vidas medias pueden llegar a ser muy grandes en ciertos regímenes de parámetros. En nuestro caso, estos estados metaestables corresponden a los estados pretermalizados discutidos en el capítulo anterior.

Esperamos entonces que este tipo de correladores funcione como una herramienta de diagnóstico de ergodicidad en la situación fuera de equilibrio que estamos considerando. En el panel izquierdo de la Fig. 5.3 mostramos el decaimiento de $C_{J}(t)$ para el caso en el que el estado inicial es $\left|\Psi_{0}\right\rangle$, variando el valor del parámetro de rotura de integrabilidad $J_{2}$ en un sistema con $L=800$ sitios y para tiempos lejos de cualquier recurrencia. Para valores grandes de $J_{2} \sim J_{1}$ (lejos de la integrabilidad) el decaimiento se desarrolla en un solo paso. Al disminuir el valor de $J_{2}$ surge un plateau entre la evolución gaussiana inicial y el decaimiento final. La extensión temporal del plateau se hace cada vez más grande a medida que disminuimos el valor de $J_{2}$, mas no aumenta suavemente sino que tiene un salto abrupto para $J_{2}=0,5 J_{1}$.

Otro factor que influencia profundamente la vida media de estos estados pretermalizados es la energía del sistema. Investigamos el comportamiento de $C_{J}(t)$ para estados iniciales con tempratura finita, es decir, estados iniciales que poseen una función de distribución de la forma

$$
n(k, 0)=\left(1+\exp \left[\left(\epsilon(k)-\epsilon\left(k_{F}\right)\right) / T\right]\right)^{-1},
$$




\section{Capítulo 5. Estados pretermalizados y comportamiento tipo vidrio en sistemas casi integrables en una dimensión}

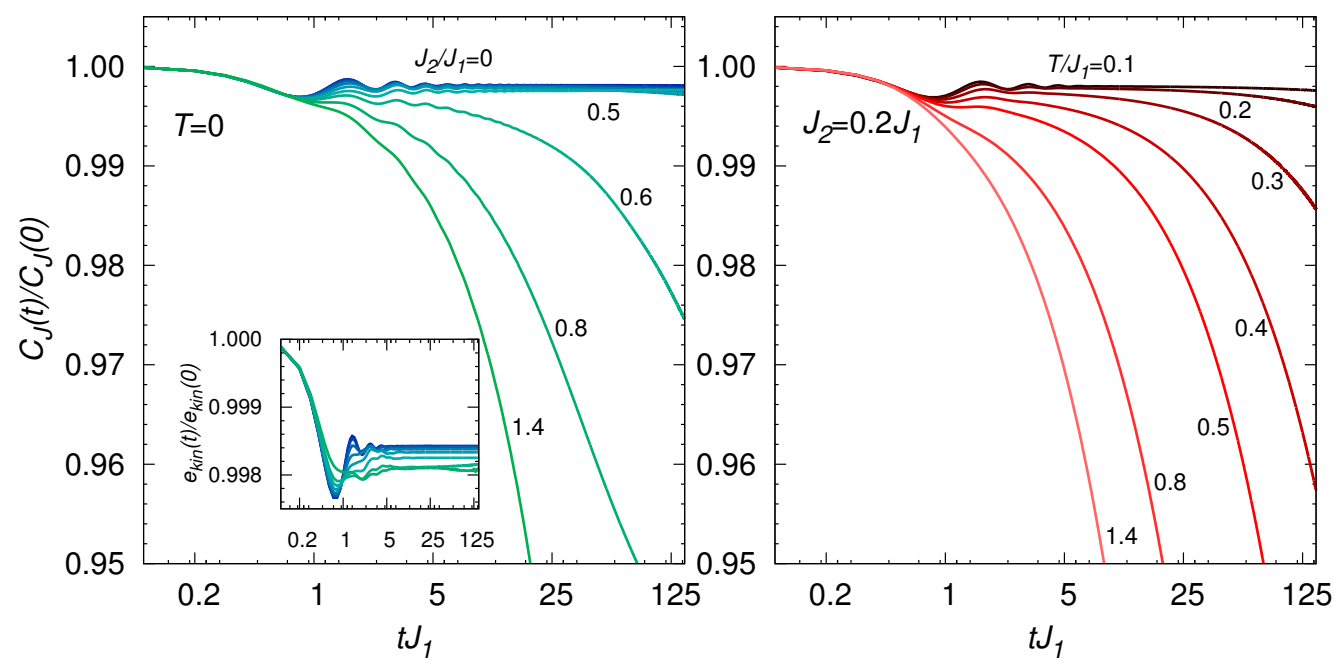

Figura 5.3: Decaimiento de la función de correlación $C_{J}(t)$. Panel izquierdo: empezando desde la condición inicial $T=0$ variando $J_{2}$, para la cual la densidad de energía es $e=0$ independientemente del valor de $J_{2}$. Inset: Relajación de la energía cinética para los mismos parámetros que en la figura principal. Panel derecho: $J_{2}=0,2 J_{1}$ fijo y se varía la temperatura $T$ del estado inicial. Nótese la escala logarítmica en el eje temporal.

en donde $T$ es la temperatura $\left(k_{B}=1\right)$. La densidad de energía del sistema

$$
e=\frac{1}{L} \operatorname{Tr}[\rho(0) H]-e_{0},
$$

con $e_{0}=\frac{1}{L}\left\langle\Psi_{0}\left|H\left(J_{1}, 0, \Delta\right)\right| \Psi_{0}\right\rangle$ la densidad de energía del estado fundamental de $H\left(J_{1}, 0, \Delta\right)$, es una función suave y monótona de $T$. En el panel derecho de la Fig. 5.3 mostramos la relajación de $C_{J}(t)$ para $J_{2}=0,2 J_{1}$ variando la temperatura de la condición inicial. El efecto de aumentar la temperatura (energía) de la condición inicial es la de hacer decrecer gradualmente la vida media de los estados pretermalizados; para energías lo suficientemente altas éstos están completamente suprimidos.Observamos también que los estados metaestables emergen en la misma escala de tiempo en la que la energía cinética del sistema $e_{\text {kin }}(t)=\left\langle H_{0}(t)\right\rangle$ satura a su valor final (véase el inset en la Fig. 5.3). Tal escala de pretermalización, $t_{\mathrm{pt}}$, resulta ser independiente del valor de $J_{2}$ y de la energía del sistema: $t_{\mathrm{pt}} J_{1} \sim 5$ en todos los casos.

\subsubsection{Dinámica de sistemas finitos y escalas de tiempo}

Para poder hacer afirmaciones cuantitativas acerca de la vida media de los estados pretermalizados nos concentraremos en sistemas más pequeños, con $L=256$, lo que nos permitirá llegar a tiempos más largos. En tal caso es necesario notar que, especialmente para tiempos largos, puede haber efectos apreciables de tamaño finito, algo que analizaremos más adelante. Vimos que en $D=1$ el salto en la distribución de momentos en $k_{F}$ no exhibe ningún 

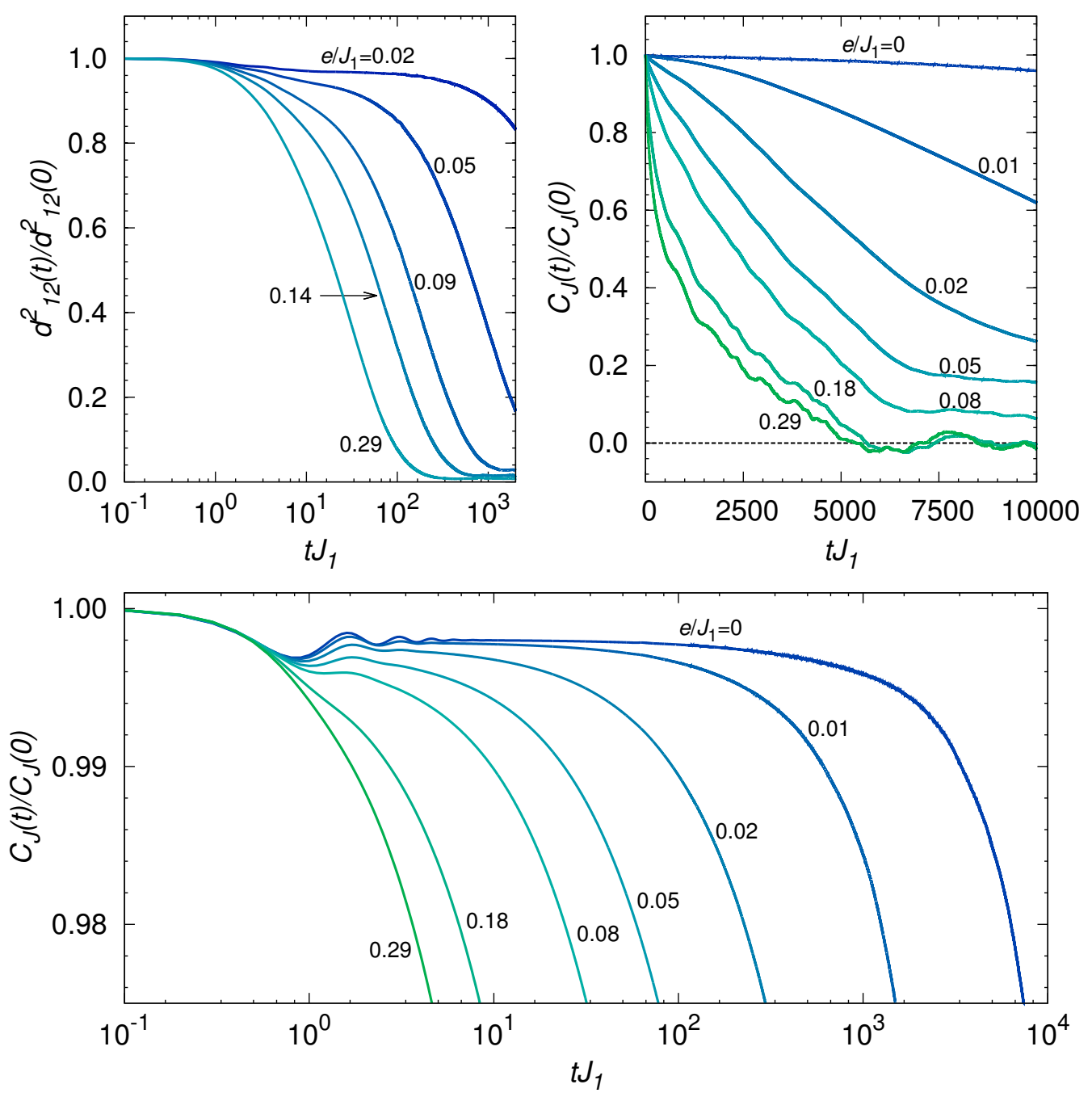

Figura 5.4: Panel superior izquierdo: Decaimiento de la distancia dinámica $d_{12}^{2}(t)$ (nótese la escala logarítmica en el eje temporal) para $J_{2}=0,2 J_{1}$ variando la energía del par de condiciones iniciales. Panel superior derecho: Decaimiento de la función de correlación $C_{J}(t)$ para $J_{2}=0,2 J_{1}$ variando la energía $e$ del estado inicial. Panel inferior: Detalle del comportamiento de $C_{J}(t) / C_{J}(0)$ para mostrar la presencia de una relajación en dos pasos y estados cuasiestacionarios con vidas medias largas. 


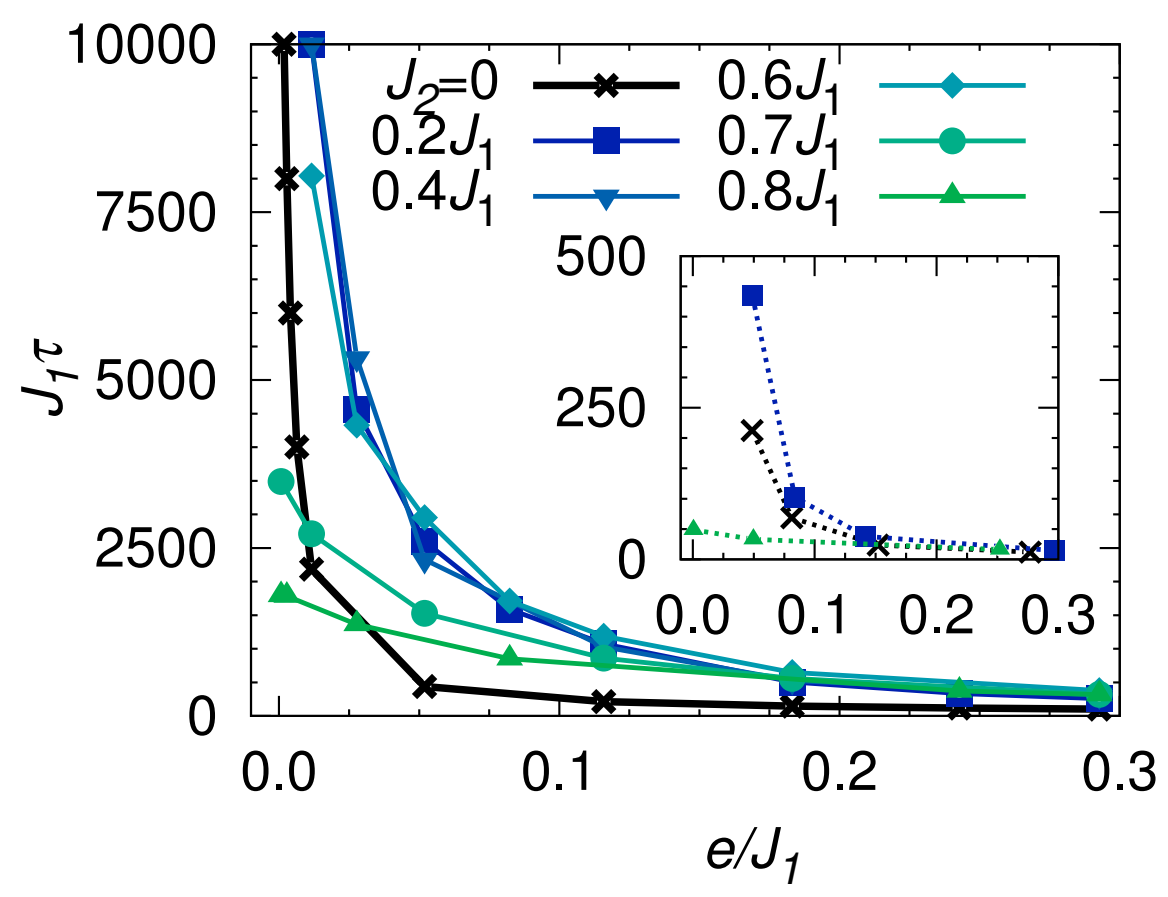

Figura 5.5: Escalas de relajación características del correlador $C_{J}(t)$ para diferentes valores de $J_{2}$ como función de la energía del estado inicial. Inset: Escalas de tiempo para la distancia dinñamica $d_{12}$ como función de la densidad de energía de las condiciones iniciales para diferentes valores representativos de $J_{2}, 0,0,2 J_{1}$ y $0,8 J_{1}$ (con los mismos símbolos que en la figura principal pero con líneas discontinuas). 
plateau. Sin embargo, las ocupaciones de los modos de momento lejos de la superficie de Fermi sí lo hacen. Para caracterizar entonces la relajación global y la aparición de estados metaestables en la distribución de momentos estudiaremos la dinámica de dos réplicas del sistema. Preparamos dos condiciones iniciales $n_{1}(k, 0)$ y $n_{2}(k, 0)$ con aproximadamente la misma energía y densidad de partículas, y definimos la distancia entre las distribuciones de momentos dependientes del tiempo

$$
d_{12}^{2}(t)=\frac{1}{L} \sum_{k}\left(n_{1}(k, t)-n_{2}(k, t)\right)^{2} .
$$

Para un sistema cuyas únicas cantidades conservadas son la energía total y el número de partíclas esta distancia debería decaer a cero para tiempos largos, ya que el estado final de la evolución está determinado únicamente por el valor de esas dos cantidades conservadas. Para sistemas con cantidades conservadas adicionales (como el modelo integrable $J_{2}=0$ ) puede saturar a una constante diferente de cero. En cualquier caso, si los dos sistemas preparados en diferentes condiciones iniciales, quedan atrapados en diferentes estados metaestables no térmicos entonces aparecerá un plateau en $d_{12}^{2}(t)$. Preparamos las condiciones iniciales perturbando ligeramente estados térmicos de fermiones libres, siempre cuidando que posean la misma energía y densidad de partículas. En el panel superior izquierdo de la Fig. 5.4 mostramos el decaimiento de $d_{12}^{2}(t)$ para $L=256$ y $J_{2}=0,2 J_{1}$, pero los resultados son similares para cualquier $J_{2}<0,5 J_{1}{ }^{2}$. Encontramos que si la energía del par de condiciones iniciales es lo suficientemente baja $d_{12}^{2}(t)$ relaja en dos pasos. La extensión del plateau aumenta al bajar la energía. En contraste, para $J_{2}>0,5 J_{1}$ encontramos relajación en un solo paso en todo el espectro de energías (algo que no mostramos explícitamente), confirmando la imagen brindada por ele análisis del correlador $C_{J}(t)$. En el panel superior derecho de la Fig. 5.4 mostramos la relajación global de la función de correlación $C_{J}(t)$ también para $J_{2}=0,2 J_{1}$. Para energías altas encontramos que decae a cero en escalas detiempo accesibles a nuestro cálculo numérico. Para bajas energías observamos un enlentecimiento muy pronunciado de la relajación asociado con la presencia de plateaus de vida media larga (ver panel inferior de la Fig. 5.4.

En la Fig. 5.5 mostramos las escalas de decaimiento de $C_{J}(t)$ para diferentes valores de $J_{2}$. Definimos la escala de tiempo de relajación $\tau$ como aquella para la cual $C_{J}(\tau) / C_{J}(0)=0,6$, para poder extraer la mayor cantidad de puntos de los datos a disposición mientras consideramos un indicador fidenigno de las escalas de relajación. Podemos apreciar que para $J_{2} \lesssim 0,5 J_{1}$ las escalas de relajación muestran un incremento abrupto para energías bajas. Para $J_{2}>0,5 J_{1}$ las escalas de tiempo de relajación prácticamente no cambian al variar la energía del estado inicial.

Para ver cómo afectan los efectos de tamaño finito a los resultados con $L=256$ debemos analizar la dependencia de las escalas de relajación con el tamaño del sistema. En la Fig. 5.6 mostramos la dependencia de las escalas de relajación de la función de correlación $C_{J}(t)$ en

\footnotetext{
${ }^{2}$ En el caso particular de $J_{2}=0$ también encontramos plateaus a tiempos cortos, pero la distancia satura a un valor no nulo.
} 


\section{Capítulo 5. Estados pretermalizados y comportamiento tipo vidrio en sistemas casi integrables en una dimensión}
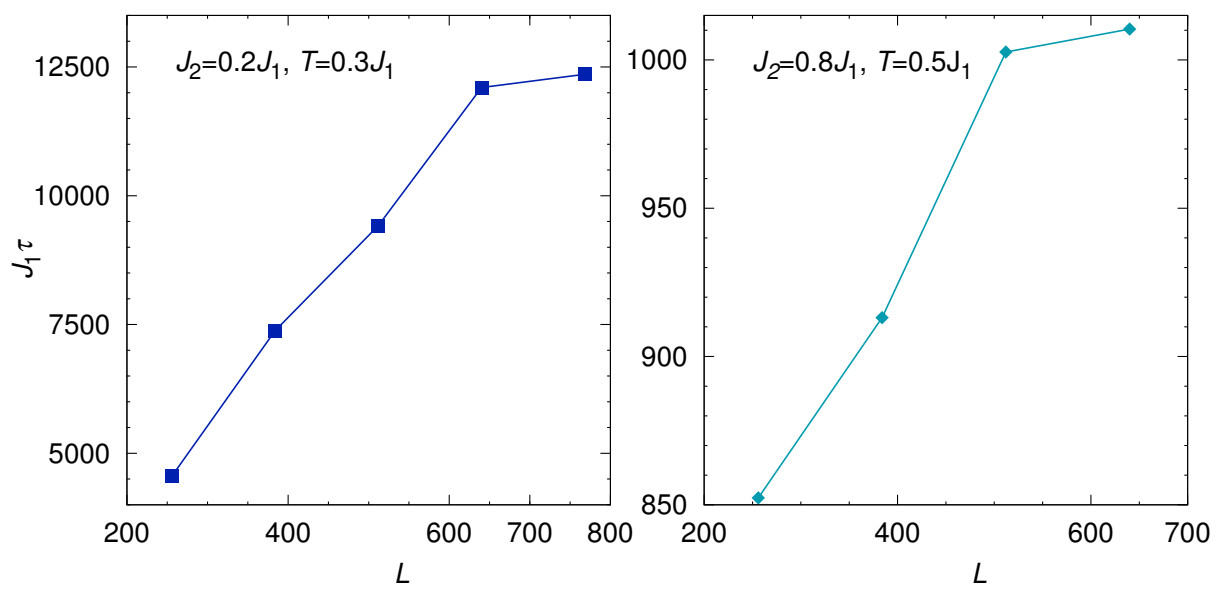

Figura 5.6: Escalas de tiempo de relajación de $C_{J}(t)$ como función del tamaño del sistema. Panel izquierdo: Para $J_{2}=0,2 J_{1}$ y $T=0,3 J_{1}$, dentro de la región de parámetros con estados metaestables con vida media larga. Panel derecho: Para $J_{2}=0,8 J_{1}$ y $T=0,5 J_{1}$, en la región de parámetros con relajación en un paso.

función del tamaño del sistema para un juego de parámetros en lo profundo de la fase "vidriosa" y para un juego de parámetros que dan lugar a relajación normal (en un paso). Observamos que las escalas de tiempo del sistema normal (vidrioso) aumentan casi linealmente con el tamaño del sistema con una pendiente $\sim 2(\sim 20)$ hasta que, alrededor de $L \sim 500(L \sim 700)$ comienza a saturar al valor del límite termodinámico.

\subsubsection{Discusión}

Puede formularse una explicación cualitativa de lo que está sucediendo. La evolución inicial rápida que se da para $t J_{1}<1$ está dominada por el dephasing de ciertos modos cuasilibres del sistema, tal y como fue explicado en los capítulos anteriores. Este régimen inicial no es sensible a los detalles de la interacción y es análogo a la expansión balística inicial en la relajación de los vidrios clásicos y de los fluidos en general. La relajación subsecuente del sistema, en cambio, queda determinada por las colisiones inelásticas. Si los canales de relajación inelásticos son escasos el sistema se queda atrapado de estados metaestables cuya vida media es una medida de la frecuencia de los eventos de scattering inelástico. Para el modelo $H\left(J_{1}, J_{2}, \Delta\right)$ puede mostrarse que tan pronto como $J_{2}$ se hace mayor que $0,5 J_{1}$ la variedad de colisiones cinéticamente permitidas (aquellas que conservan el momento y la energía cinética) crece dramáticamente [46] (ver Fig. 5.7). Tal tipo de colisiones están incluidas pero no agotan los procesos modelados por las ecuaciones de movimiento que consideramos (véase el Capítulo 4). De esta manera el número de canales de relajación aumenta drásticamente variando uno de los parámtros del sistema más allá de un umbral bien definido. Además, dado que a energías más altas hay más colisiones inelásticas posibles, la vida media de los estados metaestables se 
va suprimiendo a medida que aumentamos la energía del sistema.

Notemos que, independientemente de la integrabilidad para el modelo con $J_{2}=0$, en la región de parámetros que estamos considerando siempre estamos cerca de un modelo integrable trivial: debido al carácter perturbativo de nuestros métodos siempre estamos trabajando con una perturbación a un modelo de fermiones libres. Aun así, existen regiones en el espacio de parámetros en las que los estados pretermalizados no aparecen. Esto indica que no basta sólo con estar cerca de un punto integrable para que estos estados metaestables aparezcan, sino que las características del estado inicial también influyen. En particular, especulamos que el parámetro relevante para caracterizar el estado inicial sea la diferencia $\Delta E=\operatorname{Tr}[\rho(0) H]-\langle\Psi|H| \Psi\rangle$ entre su energía y la energía del estado fundamental $|\Psi\rangle$ del Hamiltoniano de evolución. Nuestra conjetura es que si $\Delta E$ es lo suficientemente pequeña y estamos cerca de algún punto integrable, los estados metaestables aparecerán necesariamente, sin importar mucho los detalles de la condición inicial. Creemos que, asociado con la apertura del gap para $J_{2}>0,5 J_{1}$, existe una disminución abrupta de la energía del estado fundamental de $H$, lo que hace que, aun considerand el mismo estado inicial, $\Delta E$ sea mucho más grande para $J_{2}>0,5 J_{1}$ que para $J_{2}<0,5 J_{1}$. Esto explicaría la desaparición de los estados metaestables y la pérdida de validez de las predicciones del ML. Para corroborar nuestra conjetura es necesario calcular la energía del estado fundamental de un sistema interactuante grande, lo cual es posible con técnicas numérias de equilibrio como DMRG. Desafortunadamente, esta exploración quedará relegada forzosamente a trabajos futuros.

Finalmente, notamos que la escala de tiempo característica del decaimiento de la distancia $d_{12}^{2}(t)$, al ser independientes de los detalles de la condición inicial, es una de las escalas de tiempo características del proceso de termalización [8]. Es la escala de tiempos más allá de la cual el sistema ha perdido completamente la memoria de la condición inicial al nivel de las funciones de correlación de dos puntos. Sin embargo, esto no significa que el sistema haya alcanzado el equilibrio. De hecho, encontramos que $\tau\left[C_{J}\right]$ es, al menos, un orden de magnitud mayor que $\tau\left[d_{12}\right]$ (definida de la misma manera que para $C_{J}$ ) en todos los casos en los que contábamos con datos para comparar. Esto se ilustra en el inset de la Fig. 5.5. El hecho de que observables de una partícula alcancen el comportamiento térmico mucho antes que las correlaciones de dos partículas indica que la termalización es un proceso jerárquico. La información acerca de las condiciones iniciales codificada en una función de correlación aumenta con el orden de la correlación. Nuestros resultados sugieren que, consiguientemente, las escalas de tiempo de relajación también aumentan monótonamente con el orden de la función de correlación. Sin embargo, también esperamos que para algún orden finito (pero posiblemente muy grande) las correlaciones no termalicen, reflejando la unitaridad de la dinámica cuántica. Finalmente, notamos que es para tiempos más largos que $\tau\left[d_{12}\right]$ que una descripción basada en ecuaciones cinéticas sinmemoria, como la ecuación de Boltzmann cuántica [42], está justificada [7]. 


\section{Capítulo 5. Estados pretermalizados y comportamiento tipo vidrio en sistemas casi}

integrables en una dimensión
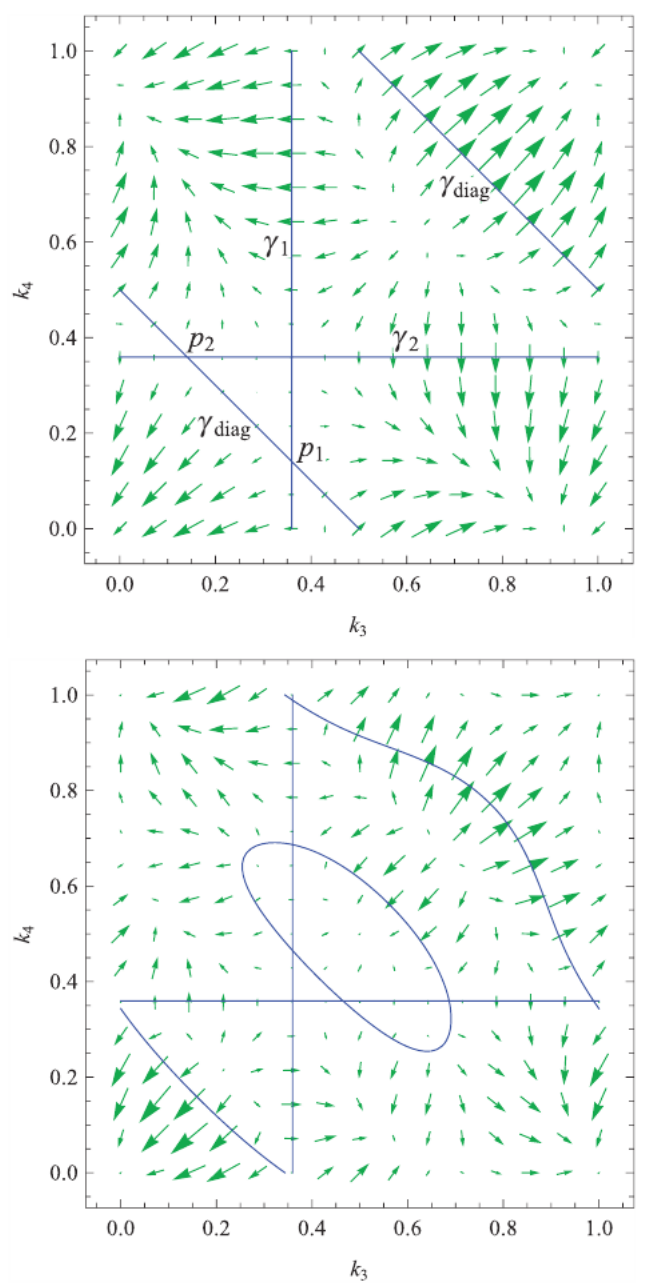

Figura 5.7: En la figura se muestran las soluciones del sistema de ecuaciones $k_{1}+k_{2}-k_{3}-k_{4}=0$, $\epsilon\left(k_{1}\right)+\epsilon\left(k_{2}\right)-\epsilon\left(k_{3}\right)-\epsilon\left(k_{4}\right)=0$, es decir, el conjunto de colisiones cinéticas permitidas, para el modelo con hopping a primeros vecinos $J_{2}=0$ (panel superior) y para un modelo con hopping a segundos vecinos $J_{2}=J_{1}$. (Se ha fijado el valor de $k_{1}$ y se ha eliminado $k_{2}$ usando la conservación del momento). En el panel superior, las rectas horizontales y verticales $\gamma_{1}$ y $\gamma_{2}$ corresponden a las soluciones triviales $k_{3}=k_{1}$ y $k_{4}=k_{1}$, respectivamente. Las líneas diagonales $\gamma_{\text {diag }}$ corresponden a $k_{3}+k_{4}=\frac{1}{2}$. Lo más interesante de esta figura es que para el modelo con hopping a segundos vecinos (panel inferior) para $J_{2}>0,5 J_{1}$ siguen existiendo las soluciones $\gamma_{1}, \gamma_{2}$ y $\gamma_{\text {diag }}$ (aunque $\gamma_{\text {diag }}$ se deforma un poco), pero además aparece un nuevo conjunto de soluciones representado por la elipse. Este nuevo conjunto de soluciones no está presente para $J_{2}<0,5 J_{1}$, es decir, es un conjunto de canales de relajación que se abren abruptamente al aumentar $J_{2}$ por encima de un valor crítico $\left(0,5 J_{1}\right)$, como está explicado en el texto. Las flechas verdes son el gradiente de la función $\epsilon\left(k_{1}\right)+\epsilon\left(k_{2}\right)-\epsilon\left(k_{3}\right)-\epsilon\left(k_{4}\right)$, que es irrelevante para nuestra discusión. Adaptado de [47]. 


\subsection{Bosones fuertemente interactuantes: Estados pretermalizados y heterogeneidad dinámica}

\subsubsection{El caso particular del modelo de Bose-Hubbard}

Existe una variedad de trabajos que reportan sobre la aparente falta de termalización en el modelo de Bose-Hubbard a interacciones fuertes. Comenzando con el trabajo de Kollath et al. [79], en donde, usando t-DMRG se mostró que para interacciones lo suficientemente fuertes las correlaciones de pocos puntos alcanzaban un estado cuasiestacionario que no era compatible con el equilibrio térmico, varios estudios se han enfocado en este sistema [127, 21]. En la Ref. [21] se mostró que para interacciones fuertes el sistema se queda atrapado en estados metaestables inhomogéneos con una vida media muy larga. Se mostró también que tal fenómeno [21] estaba asociado con una localización dinámica en el espacio de Hilbert del sistema. El modelo de Bose-Hubbard es una de los modelos que ha sido realizado con más frecuencia en experimentos con átomos ultrafríos [11]. En particular, algunos experimentos con condensados de Bose-Einstein inhomogéneos han demostrado que el sistema exhibe una pronunciada separación de escalas de tiempo y una termalización lenta para interacciones fuertes [99, 66, 93].

Creemos que la emergencia de estados pretermalizados con vidas medias muy grandes está detrás de esta aparente falta de termalización para interacciones fuertes. De hecho, el modelo de Bose-Hubbard es integrable para interacciones infinitamente fuertes (el límite atómico, ver más abajo), lo cual constriñe fuertemente la dinámica cuando las interacciones son fuertes. Sin embargo, es posible que efectos de tamaño finito y no sólo la pretermalización sean las causas de algunos de los fenómenos observados, en particular, en la Ref. [79], en la que se consideraron tamaños de sistemas muy pequeños $(L=18)$ para los cuales la ETH falla a una distancia finita de un punto integrable [121].

Respecto al aspecto técnico de esta sección, como ya hemos mencionado, utilizaremos una técnica semiclásica que describiremos más adelante. En [134] Srednicki introdujo la ETH a partir de un razonamiento semiclásico. Desde entonces, las consideraciones semiclásicas han sido muy importantes para nuestro entendimiento de la termalización cuántica. Recientemente Cosme et. al [35] hicieron una contribución en esta dirección usando la TWA aplicada a un modelo de Hubbard bosónico de dos sitios pero de varias bandas. Dado que la TWA permite considerar las correcciones cuánticas de primer orden a la acción clásica [113] la conexión entre la dinámica cuántica y el límite clásico se vuelve explícita. En particular, en [35] se mostró que la termalización del sistema cuántico está íntimamente relacionada con la ergodicidad de las trayectorias clásicas. Esta idea también se encuentra implícita en estudios previos, como [73] y [23] en donde la falla en la termalización para interacciones fuertes fue relacionada con las propiedades caóticas del límite de campo medio (trayectorias clásicas). Más precisamente se demostró que el exponente de Lyapunov de las trayectorias clásicas asociadas con las soluciones de las ecuaciones de campo medio está suprimido para interacciones fuertes. 


\section{Capítulo 5. Estados pretermalizados y comportamiento tipo vidrio en sistemas casi integrables en una dimensión}

Nuestra contribución a este problema consiste en considerar el problema de la aparente falta de termalización en el modelo de Hubbard bosónico desde la perspectiva semiclásica de la TWA. Para densidades medias grandes, este método permite acceder a la dinámica cuántica realizando promedios pesados sobre ensambles de trayectorias clásicas. Mostraremos explícitamente que en este caso también los estados pretermalizados pueden tener una vida media enorme que crece exponencialmente al aumentar la intensidad del acoplamiento, lo que evita que el sistema relaje al equilibrio térmico en una escala de tiempos razonable. Además, encontramos que la emergencia de estados pretermalizados con vidas medias largas está relacionado estrechamente con la aparición de características no ergódicas y «vidriosas» en las trayectorias de campo medio asociadas con la dinámica cuántica. Más específicamente, encontramos que los ensambles de trayectorias de campo medio sufren una transición de fase dinámica entre una fase activa y una inactiva, en completa analogía a lo que se observa en modelos clásicos de vidrios [62, 48, 49,62]. Esta transición está asociada con un fenómeno notable, la heterogeneidad dinámica [9].

\subsubsection{Dinámica cuántica: Resultados de la TWA}

Consideremos el modelo de Bose-Hubbard en una dimensión con Hamiltoniano $H=H_{0}+H_{\text {int }}$ :

$$
\begin{aligned}
H_{0} & =-J \sum_{j=1}^{L}\left(a_{j}^{\dagger} a_{j+1}+a_{j+1}^{\dagger} a_{j}\right), \\
H_{\text {int }} & =U \sum_{j=1}^{L} n_{j} n_{j},
\end{aligned}
$$

en donde $L$ es el número de sitios en la cadena, los operadores a $a$ son operadores bosónicos canónicos y $\hat{n}_{j}=a_{j}^{\dagger} a_{j}$. Consideraremos en este caso también condiciones de contorno periódicas, $a_{L+m}^{\#}=a_{m}^{\#}$. Analizaremos la dinámica generada por(5.10) empezando desde una estado inicial inhomogéneo descripto por la matriz densidad $\rho_{0}$, que especificaremos más adelante. Nos enfocaremos en la relajación del perfil de densidad $\left\langle\hat{n}_{j}(t)\right\rangle=\operatorname{Tr}\left[\rho_{0} e^{i H t} n_{j} e^{-i H t}\right]$ $(\hbar=1)$. El perfil de densidad térmico viene dado por $\left\langle\hat{n}_{j}\right\rangle_{\text {th }}=N_{a} / L=N$ para todo $j$, en donde $N_{a}$ es el número total de bosones. El modelo de Hubbard bosónico es integrable en dos límites triviales, para $U=0$ (gas no interactuante) y para interacciones infinitamente fuertes, $J=0$ (el límite atómico).

Cuando el número medio de bosones por sitio $N$ es alto la dinámica del modelo de BoseHubbard puede ser estudiada usando la TWA [114]. Para calcular la dependencia temporal de los valores de expectación en la TWA debemos considerar la solución a las ecuaciones de movimiento clásicas asociadas con el Hamiltoniano cuántico (5.10). Éstas son las ecuaciones de Gross-Pitaevskii en la red [114],

$$
i \frac{d \psi_{j}(t)}{d t}=-J\left(\psi_{j+1}(t)+\psi_{j-1}(t)\right)+2 U \psi_{j}(t)\left|\psi_{j}(t)\right|^{2} .
$$


en donde los campos clásicos están normalizados al número de partículas $\sum_{j=1}^{L}\left|\psi_{j}(t)\right|^{2}=N_{a}$. El valor de expectación de un dado operador $\hat{\Omega}$ a tiempo $t,\langle\hat{\Omega}(t)\rangle \Omega$ puede ser calculado promediando el observable clásico correspondiente $\Omega$ sobre un ensamble de condiciones iniciales pesado con la transformada de Wigner de la matriz densidad inicial $\rho_{0}$

$$
\langle\hat{\Omega}(t)\rangle=\int d \psi_{0}^{*} d \psi_{0} p\left(\psi_{0}, \psi_{0}^{*}\right) \Omega_{\mathrm{cl}}\left(\psi(t), \psi^{*}(t)\right),
$$

en donde la transformada de Wigner $p$ viene definida por

$$
p\left(\psi_{0}, \psi_{0}^{*}\right)=\int d \eta_{0}^{*} d \eta_{0}\left\langle\psi_{0}-\frac{\eta_{0}}{2}\left|\rho_{0}\right| \psi_{0}+\frac{\eta_{0}}{2}\right\rangle e^{-\left|\psi_{0}\right|^{2}-\frac{1}{4}\left|\eta_{0}\right|^{2}} e^{\frac{1}{2}\left(\eta_{0}^{*} \psi_{0}-\eta_{0} \psi_{0}^{*}\right)},
$$

en donde $\left|\psi_{0} \pm \frac{\eta_{0}}{2}\right\rangle$ es un estado coherente con autovalor $\psi_{0} \pm \frac{\eta_{0}}{2}$ con respecto al operador de aniquilación $a$. Para alivianar la notación hemos omitido el índice de sitio $j$. La medida es

$$
d \psi_{0}^{*} d \psi_{0}=\prod_{j=1}^{L} d \psi_{j}^{*}(0) d \psi_{j}(0) .
$$

Más adelante mostraremos como implementar la integración sobre condiciones iniciales utilizando el método Monte Carlo, es decir, muestreando de forma aleatorea la función de Wigner del estado inicial $p\left(\psi_{0}, \psi_{0}^{*}\right)$.

La correspondencia entre observables cuánticos y clásicos puede formularse fácilmente usando la representación de Bopp [113], según la cual se deben hacer las siguientes asignaciones

$$
\begin{gathered}
\hat{a}^{\dagger} \rightarrow \psi^{*}-\frac{1}{2} \frac{\partial}{\partial \psi}, \\
\hat{a} \rightarrow \psi+\frac{1}{2} \frac{\partial}{\partial \psi^{*}},
\end{gathered}
$$

de manera tal que

$$
\hat{n}_{j} \rightarrow\left|\psi_{j}\right|^{2}-\frac{1}{2} .
$$

La TWA es el orden dominante en una aproximación en un parámetro pequeño que mide las desviaciones de la clasicalidad [113]. En el caso particular de los bosones interactuantes este parámetro es $1 / N$, la inversa de la densidad media de bosones. La TWA es una aproximación controlada en el sentido de que es posible calcular correcciones de orden superior que toman la forma de perturbaciones estocásticas sobre las trayectorias clásicas [113]. Sin embargo, como cualquier aproximación, sea controlada o no, hay ciertos regímenes de parámetros en los que funciona mejor que en otros. Para definir en qué situaciones esperamos que la aproximación sea precisa introducimos el parámetro de no linealidad $\lambda=\frac{U N}{J}$ que es el cociente entre la energía potencial típica por sitio y la energía cinética típica por sitio [23]. Cuando las interacciones son lo suficientemente grandes, $\lambda \sim N^{2}$, el sistema (en equilibrio) 


\section{Capítulo 5. Estados pretermalizados y comportamiento tipo vidrio en sistemas casi integrables en una dimensión}

sufre una transición de fase a una fase aisladora de Mott. En la vecindad de la transición las fluctuaciones cuánticas se hacen grandes y no podemos esperar que la TWA funcione bien en este caso, es decir, las correcciones de segundo o mayor orden se vuelve relevantes en este régimen. Sin embargo, hay un amplio intervalo, $\lambda \lesssim N^{2}(U / J \lesssim N)$ en el cual el estado fundamental del sistema es un superfluido (débil o fuertemente interactuante dependiendo de si $\lambda<1$ o $\lambda>1$ respectivamente) en el cual podemos esperar que la TWA sea una buena aproximación a la dinámica. Trabajaremos en ese régimen.

En paralelo, la elección de las condiciones iniciales también es relevante para la precisión de los resultados. Estudiaremos dos tipos de condiciones iniciales, estados iniciales coherentes (superfluidos) y estados iniciales de Fock (aisladores de Mott). El estado coherente representa la condición inicial que está mas cerca de una condición inicial clásica perfectamente definida y por lo tanto esperamos que la TWA capture la dinámica correctamente. La función de Wigner es

$$
p_{C}\left(\psi_{0}, \psi_{0}^{*}\right)=\prod_{j=1}^{L} \frac{2}{\pi} e^{-2\left|\psi_{j}-N_{j}\right|^{2}},
$$

en donde $N_{j}=\left\langle\hat{n}_{j}(0)\right\rangle$. Esta función de Wigner es una verdadera distribución de probabilidades y puede ser muestreada eficientemente como $\psi_{j}=\sqrt{N_{j}}+\frac{1}{2}\left(\eta_{1}+i \eta_{2}\right)[35,107]$ en donde $\eta_{1}$ y $\eta_{2}$ son dos variables aleatorias gaussianas reales con correlaciones $\overline{\eta_{j}}=0 \mathrm{y}$ $\overline{\eta_{j} \eta_{k}}=\delta_{j k}[35,107]$.

El estado inicial de Fock es el menos clásico en el sentido de que las fases iniciales están completamente indefinidas. la función de Wigner en este caso es

$$
p_{F}\left(\psi_{0}, \psi_{0}^{*}\right)=\prod_{j=1}^{L} 2 e^{-2\left|\psi_{j}\right|^{2}} L_{N_{j}}\left(4\left|\psi_{j}\right|^{2}\right),
$$

en donde $L_{N}(x)$ es el polinomio de Laguerre de $N$-ésimo orden. Desafortunadamente, esta $p$ no es definida positiva y para $N$ grande exhibe un comportamiento oscilatorio muy pronunciado. Este hecho hace que la integración Monte Carlo necesaria para calcular los observables cuánticos converja mucho más lentamente. Para eludir esta complicación aproximaremos esta función de Wigner por productos de distribuciones que reproduzcan los primeros momentos de los polinomios de Laguerre. La manera más simple de hacerlo es reproducir sólo el primer momento reemplazando cada factor $p_{N_{j}}\left(\psi_{j}^{*}, \psi_{j}\right)$ por una delta de Dirac $\delta\left(\left|\psi_{j}\right|^{2}-\frac{1}{2}-N_{j}\right)$ y promediar sobre fases aleatorias [112, 35, 107]. Una aproximación un poco menos cruda sería considerar distribuciones con dos momentos, es decir, una gaussiana $\frac{2}{\sqrt{2 \pi}} e^{-2\left(\left|\psi_{j}\right|^{2}-\frac{1}{2}-N_{j}\right)^{2}}$. Nos referiremos a estas dos aproximaciones como las funciones de Wigner delta y gaussiana, $p_{\delta}$ y $p_{\mathrm{g}}$ respectivamente. Hemos verificado que en los casos particulares que investigamos ambas aproximaciones proveen de resultados muy similares. Usaremos la $p_{\delta}$ por conveniencia computacional. Para el estado inicial de Fock se encontró que, en el peor de los casos, los resultados de la TWA se desvían cuantitativamente de la solución exacta (en los casos en las que esta está disponible) para tiempos mayores que $t_{c}=J / U$ [114]. Sin 

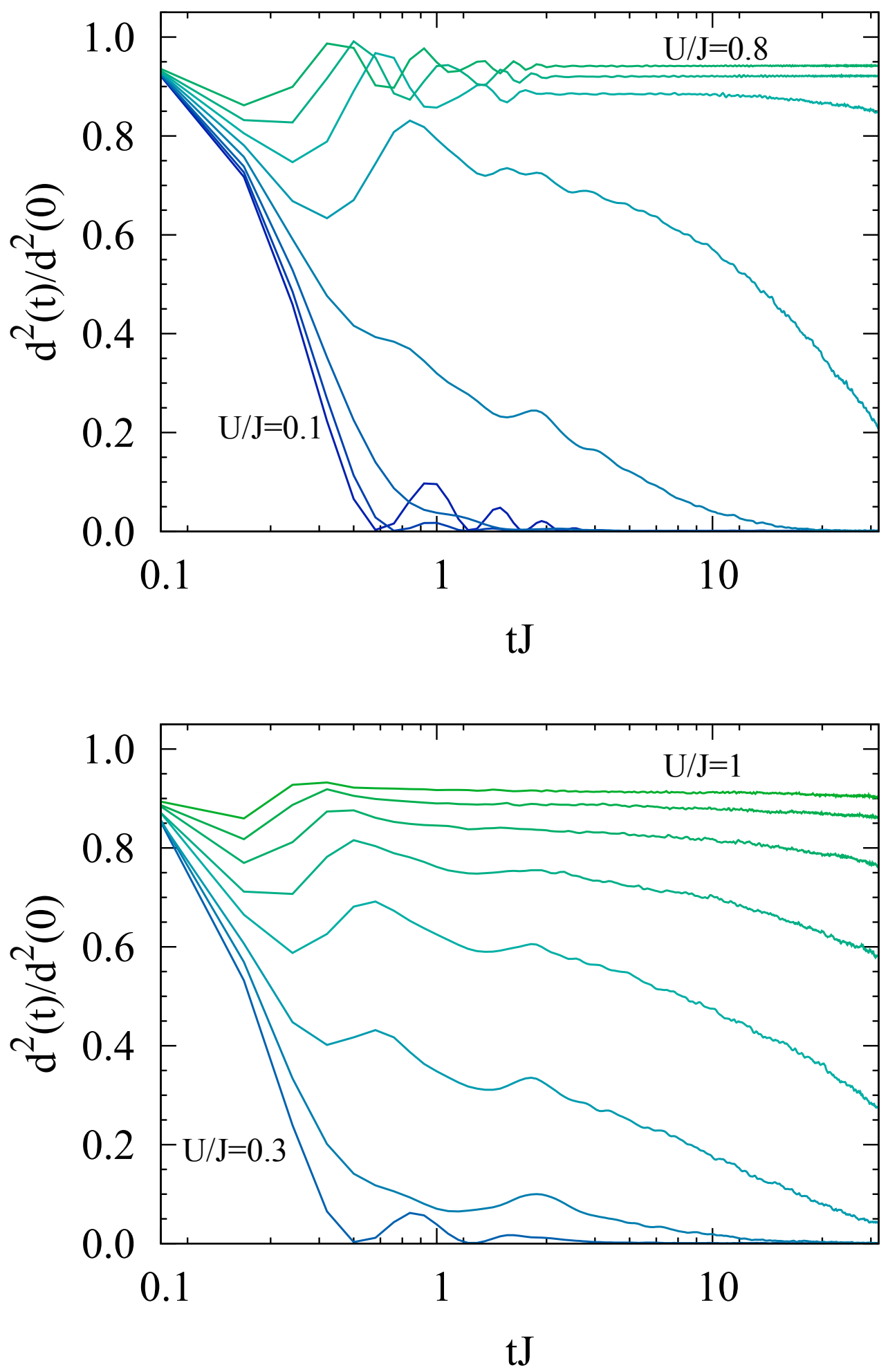

Figura 5.8: Distancia dinámica $d(t)$ como función del tiempo para el estado inicial de Fock (arriba) y coherente (abajo). Curvas diferentes corresponden a diferentes intensidades de interacción $U / J$. 


\section{Capítulo 5. Estados pretermalizados y comportamiento tipo vidrio en sistemas casi integrables en una dimensión}

embargo, las predicciones de la TWA se mantienen cualitativamente válidas para escalas de tiempo más grandes.

Ahora investigaremos la dinámica cuántica del perfil de densidad. Elegimos los estados iniciales coherentes o de Fock de tal manera que el perfil de densidad inicial sea $(20,0,20,0, \ldots)$ : una alternación de sitios vacíos y altamente ocupados. Con esta elección $N=10$, es decir, la intensidad de interacción relacionada con la transición al aislador de Mott es $U / J \sim 10$. El sistema está en un anillo de tamaño $L=30$. Estos estados iniciales pueden ser experimentalmente relevantes para experimentos con átomos fríos cargados en redes ópticas [138]. Para calcular la dinámica muestreamos $10^{3}$ condiciones iniciales diferentes tanto para el estado coherente como para el estado de Fock, aunque hemos realizado corridas con hasta $10^{4}$ realizaciones para verificar la convergencia de los resultados. Para cuantificar la relajación global del perfil de densidades a su configuración térmica introducimos la distancia dinámica:

$$
d^{2}(t)=\frac{1}{L} \sum_{j=1}^{L}\left(\left\langle\hat{n}_{j}(t)\right\rangle-N\right)^{2}
$$

Claramente, el equilibrio térmico implica $d^{2}(t)=0$. En la Fig. 5.8 mostramos el decaimiento de $d^{2}(t)$ tanto para un estado inicial de Fock como para un estado inicial coherente para diferentes valores del acoplamiento $U$. En ambos casos, para acoplamientos débiles el sistema termaliza rápidamente en una escala de tiempos del orden de un hopping [138, 133]. A medida que aumentamos la intensidad del acoplamiento emerge un estado metaestable entre la relajación inicial y el decaimiento final al equilibrio. La vida media de tal estado metaestable se incrementa con la intensidad del acoplamiento hasta que se vuelve más grande que el tiempo de simulación máximo. Para el estado inicial de Fock el incremento en la vida media del estado metaestable es más abrupto. En ambos casos, el perfil de densidad del estado metaestable está más cerca del estado inicial a medida que incrementamos la intensidad de la interacción.

En la Fig. 5.9 mostramos la relajación de la energía cinética $e_{\text {kin }}(t)=\operatorname{Tr}\left[\rho_{0} e^{i H t} H_{0} e^{-i H t}\right]$ para 1 condición inicial coherente y para diferentes valores del acoplamiento. Para el estado inicial de Fock obtenemos cualitativamente los mismos resultados. Observamos que para interacciones débiles $U / J \lesssim 0,1$ la energía cinética pretermaliza en un escala de tiempos $t_{\mathrm{pt}}^{\text {weak }} \sim 10 J^{-1}$. Para interacciones intermedias $0,2 \lesssim U / J \lesssim 0,7$ la energía cinética también tiende a estabilizarse en una constante pero con un tiempo de relajación más largo, del orden de $t_{\mathrm{pt}}^{\mathrm{int}} \sim 30 J^{-1}$ (int se refiere a rango intermedio de acoplamientos). Mientras que para acoplamientos fuertes $U / J \gtrsim 0,7$ la escala de pretermalización es $t_{\mathrm{pt}}^{\text {strong }} \sim 3 J^{-1}$. La variedad de escalas de pretermalización puede entenderse a partir del hecho de que los modos cuasilibres detrás de la relajación por dephasing son cualitativamente diferentes para interacciones débiles o fuertes . Para interacciones débiles los modos cuasilibres están relacionados con los modos de momento que diagonalizan $H_{0}$ y están completamente deslocalizados en el espacio. Para interacciones fuertes, los modos cuasilibres están relacionados con los modos que diagonalizan $H_{\text {int }}$ y son excitaciones completamente localizadas. Para interacciones 


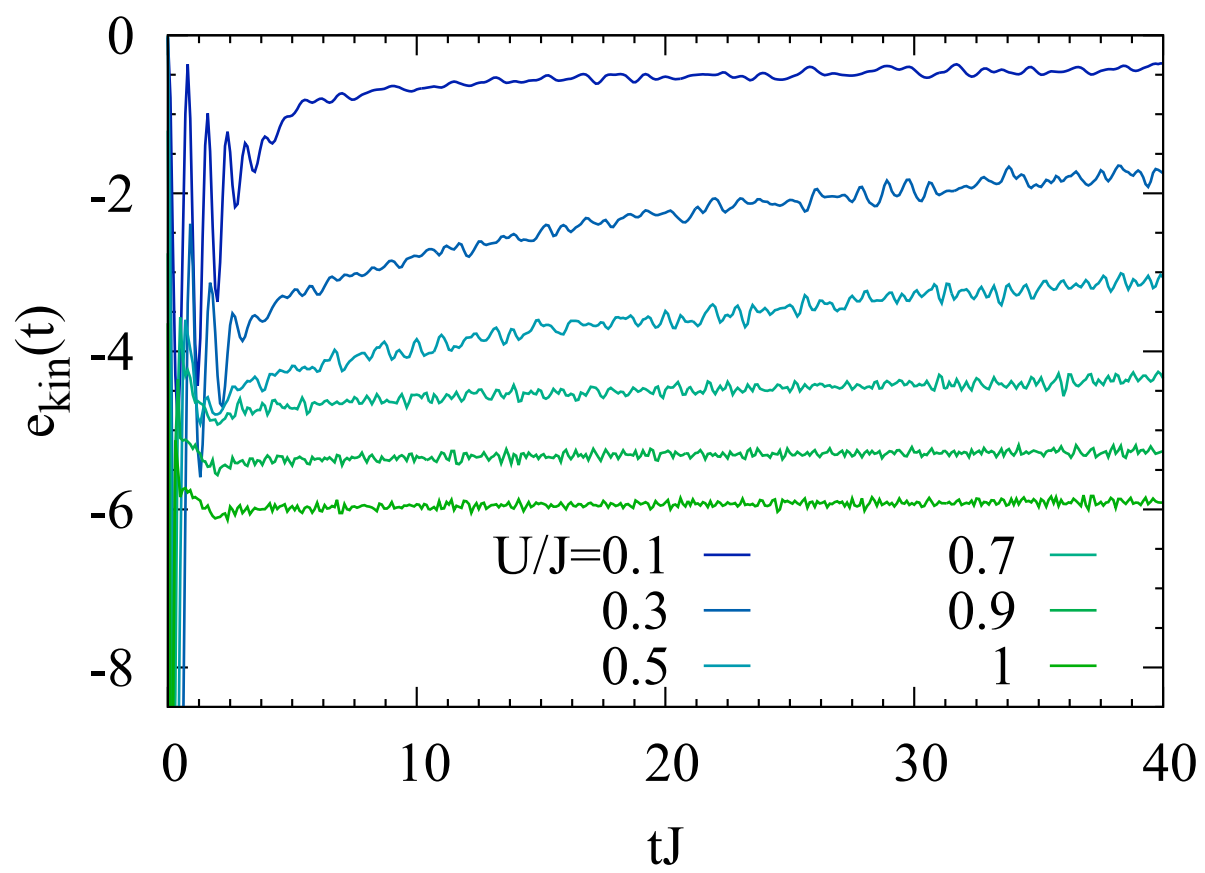

Figura 5.9: Relajación de la energía cinética para la condición inicial estado coherente. Curvas diferentes corresponden a diferentes intensidades de la interacción $U / J$. Las curvas están desplazadas verticalmente para mejorar la claridad de gráfico. 


\section{Capítulo 5. Estados pretermalizados y comportamiento tipo vidrio en sistemas casi integrables en una dimensión}

intermedias existe un régimen realmente no trivial para el cual los modos cuasilibres no están ni completamente localizados ni completamente deslocalizados. Esto sugiere que estos modos, que describen la dinámica de tiempos cortos, en los tres casos son completamente diferentes. Sin embargo, un hecho notable es que para interacciones fuertes el tiempo de pretermalización es el mismo sin importar el valor específico del acoplamiento, mientras que los tiempos de relajación del perfil de densidad muestran grandes variaciones (véase la Fig. 5.10) debido a la presencia de estados metaestables.

La transición entre termalización rápida y el régimen dominado por los estados metaestables ocurre en intervalos del acoplamiento diferentes para las dos condiciones iniciales. Para obtener la escala de decaimiento de $d^{2}(t)$ ajustamos una exponencial a la cola del decaimiento. En la Fig. 5.10 mostramos los resultados para la escala de decaimiento para ambas condiciones iniciales. Encontramos que las escalas de decaimiento aumentan en varios órdenes de magnitud cuando la intensidad del acoplamiento aumenta en un pequeño intervalo.

Mencionamos que, si bien no hemos realizado un análisis detallado de la dependencia de las escalas de tiempo para la relajación con el tamaño del sistema, algunos resultados preliminares muestran que los efectos de tamaño finito son prácticamente despreciables para los valores de $L$, las escalas de tiempo y los observables considerados aquí.

El centro de la región de transición se encuentra alrededor $\operatorname{de} U / J \simeq 0,7$ para el estado inicial de Fock y $U / J \simeq 1$ para el estado coherente. La emergencia de estados pretermalizados con una vida media tan grande puede obstaculizar completamente la observación del estado de equilibrio térmico y ciertamente está detrás de la aparente falta de termalización observada en algunos trabajos sobre este modelo [79, 20]. Esta situación es parecida a la de los vidrios, sistemas que muestran la típica relajación en dos pasos debido a que se quedan atrapados en estados metaestables con una vida media que puede ser astronómica para densidades lo suficientemente altas o temperaturas lo suficientemente bajas. Más adelante describiremos una relación más cuantitativa entre la emergencia de estados metaestables con vidas medias grandes y la física de los vidrios al analizar las propiedades de las trayectorias de campo medio asociadas con la dinámica cuántica.

\subsubsection{Propiedades de las soluciones clásicas}

Como se ha mencionado anteriormente, en la Ref. [35] fue propuesto que la capacidad de un sistema cuántico de termalizar está relacionado con la ergodicidad de las trayectorias clásicas del modelo de campo medio asociado, una idea que también está implícita en estudios previos tales como [73] y [23]. En esta sección mostraremos que la emergencia de estados pretermalizados con vida media grande está correlacionada con la falta de ergodicidad de las trayectorias clásicas que son utilizadas para construir la dinámica cuántica.

Para introducir la discusión cualitativamente, en la Fig. 5.15 mostramos un muestreo estroboscópico de la posición en el espacio de fases del campo $\psi_{1}$ para dos acoplamientos diferentes. 
5.2. Bosones fuertemente interactuantes: Estados pretermalizados y heterogeneidad dinámica
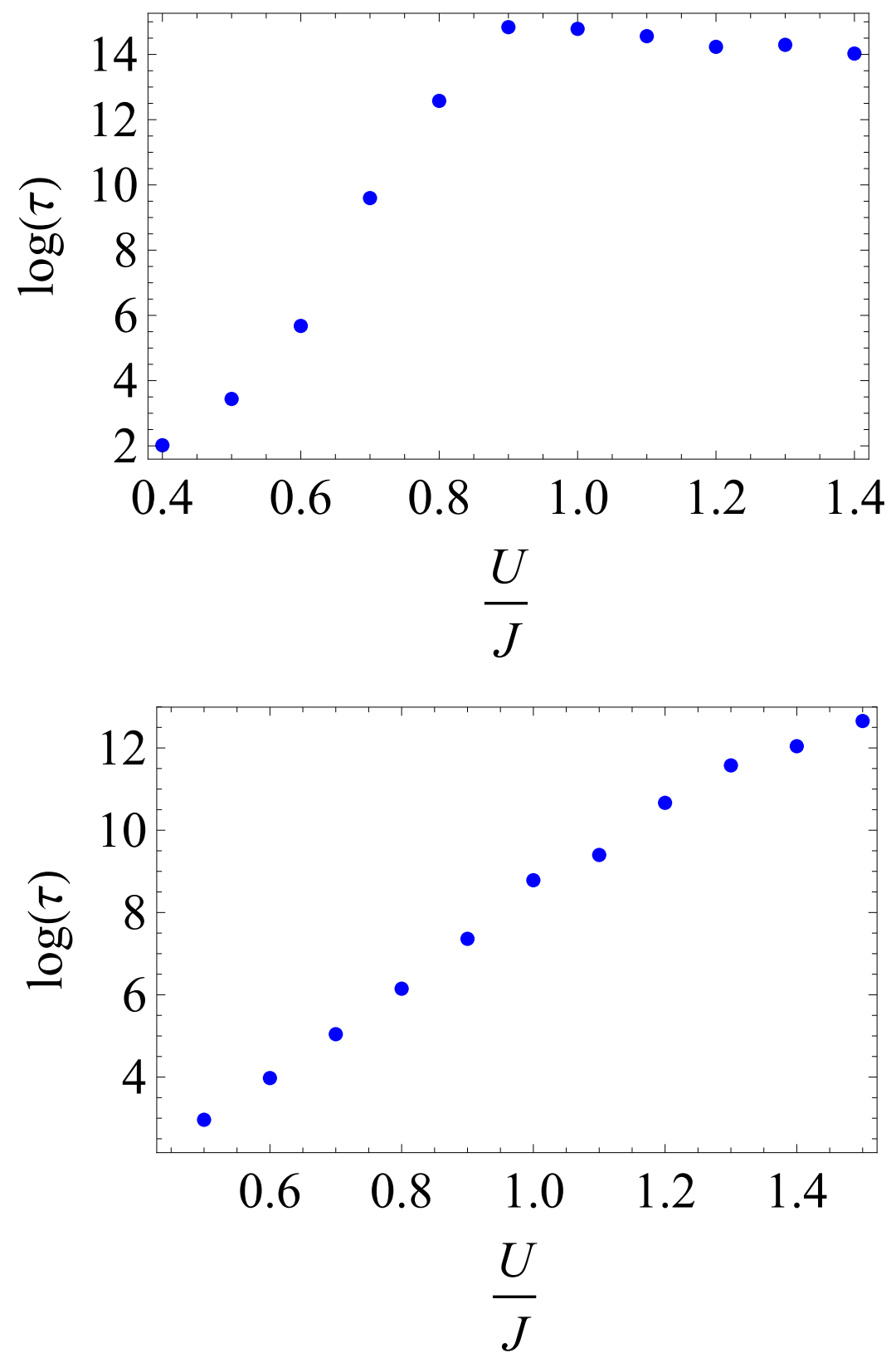

Figura 5.10: Logaritmo del tiempo de relajación como función de $U / J$ para el estado inicial de Fock (arriba) and coherente (abajo). 
Capítulo 5. Estados pretermalizados y comportamiento tipo vidrio en sistemas casi integrables en una dimensión
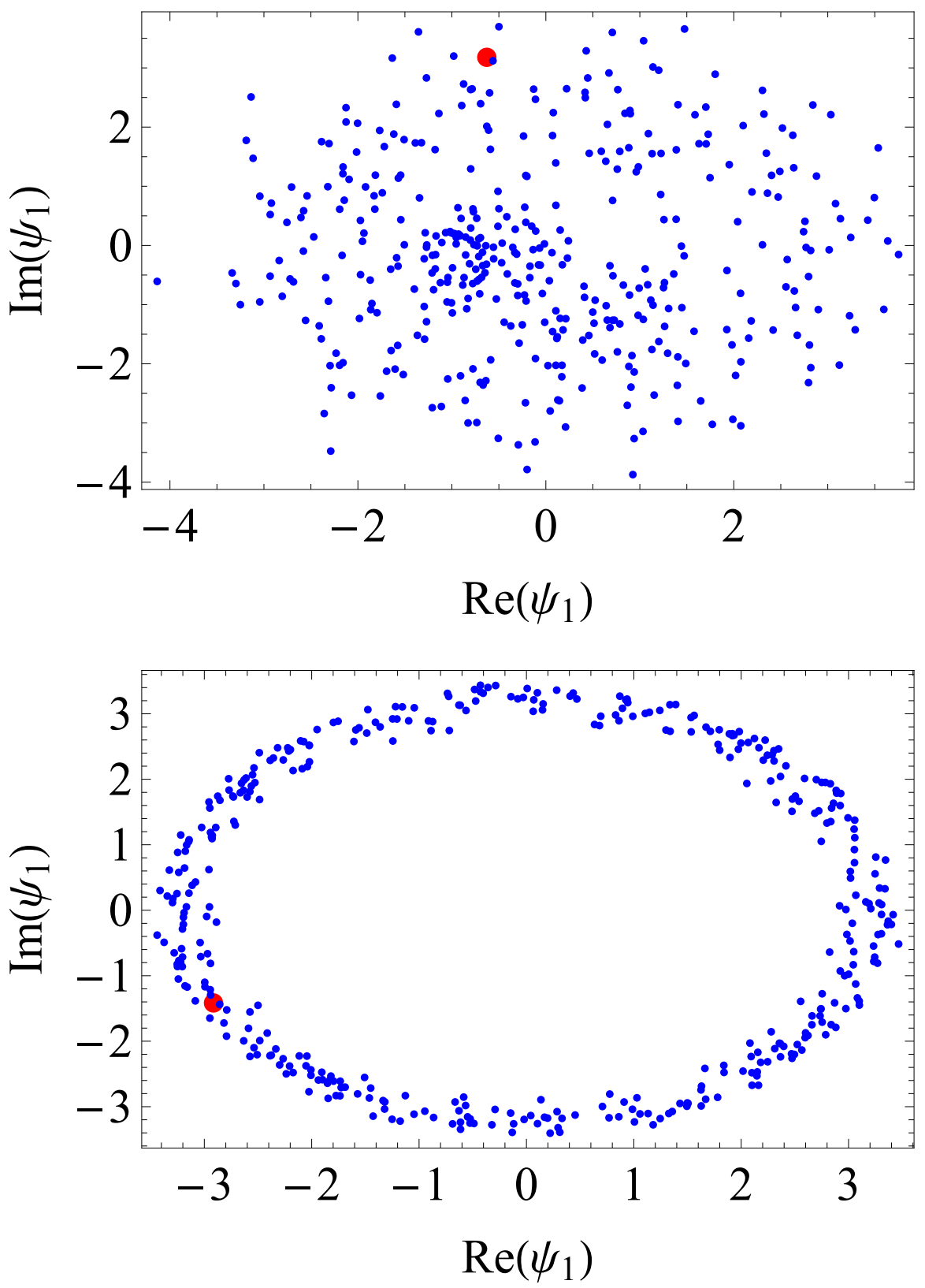

Figura 5.11: Posición en el espacio de fases $\operatorname{Re}\left(\psi_{1}\right)$ vs. $\operatorname{Im}\left(\psi_{1}\right)$ del campo en el primer sitio observada en intervalos de $J \Delta t=0,1$ para $J t_{\text {obs }}=40, U / J=0,5$ (arriba) y $U / J=1,5$ (abajo). El punto rojo corresponde a la configuración inicial. Se eligió una fase inicial al azar para cada uno de los sitios. 
Podemos ver que para acoplamientos débiles $(U=0,5)$ el sistema explora todo el espacio de fases disponible mientras que para acoplamientos grandes $(U=1,5)$ se queda atrapado en un anillo de espesor finito en el plano $\operatorname{Re}\left(\psi_{1}\right)$ vs. $\operatorname{Im}\left(\psi_{1}\right)$. Un comportamiento similar se observa al analizar el comportamiento de la trayectoria en el espacio de fases de otros sitios. Volviendo a la Fig. 5.8 podemos comprobar que, de hecho, para $U=0,5$ el perfil de densidades ya ha termalizado para $t J=40$ mientras que está todavía atrapado en un estado metaestable para $U=1,5$.

Adicionalmente, señalamos que la fase de los campos $\theta_{j}(t)=\arctan \left(\operatorname{Im}\left[\psi_{j}(t)\right] / \operatorname{Re}\left[\psi_{j}(t)\right]\right)$ resulta ser siempre ergódica, dado que durante la evolución dinámica visita todos los valores entre 0 y $2 \pi$, sin importar el valor del acoplamiento. Este hecho también queda ilustrado en la Fig. 5.11 en la cual, incluso para acoplamientos fuertes, la fase del campo $\psi_{1}$ explora todos los valores posibles. La falta de ergodicidad está codificada entonces en la dinámica de la densidad de partículas en cada sitio $n_{j}(t)=\left|\psi_{j}(t)\right|^{2}$.

Realizaremos a continuación un análisis más detallado y cuantitativo de las propiedades de las trayectorias clásicas. Para caracterizarlas introducimos la movilidad $K$ de cada trayectoria

$$
K[\psi(t)]=\Delta t \sum_{t=0}^{t_{\mathrm{obs}}} \sum_{j=1}^{N}\left(\left|\psi_{i}(t+\Delta t)\right|^{2}-\left(\left|\psi_{i}(t)\right|^{2}\right)^{2}\right.
$$

en donde $t_{o b s}$ es el tiempo máximo de observación, es decir, la extensión de las trayectorias y $\Delta t$ es un cutoff ultravioleta impuesto para ignorar pequeñas oscilaciones dejando solamente el desplazamiento real en el espacio de fases. $K[\psi(t)]$ es una cantidad extensiva en el tiempo que típicamente va a ser grande para trayectorias ergódicas y pequeña para trayectorias no ergódicas, tal como puede inferirse de la Fig. 5.15. Por lo tanto, $K[\psi(t)]$ puede ser utilizado como un parámetro de orden para distinguier trayectorias ergódicas (móviles) y no ergódicas (baja movilidad).

Dado que estamos interesados en evaluar las propiedades de las trayectorias que son más relevantes para la dinámica cuántica, introduciremos una medida en el espacio de trayectorias clásicas dada por la función de Wigner del estado cuántico inicial que deseamos considerar. En el caso del estado coherente, la medida $p_{C}\left(\psi_{0}, \psi_{0}^{*}\right)$ está bien definida mientras que en el caso del estado aislador de Mott debemos elegir cualquier distribución bien definida que aproxime a $p_{F}\left(\psi_{0}, \psi_{0}^{*}\right)$, tal como $p_{\delta}$ o $p_{\mathrm{g}}$. Por simplicidad, nos restringiremos en el análisis subsiguiente al caso de un estado coherente.

En la Fig. 5.12 mostramos el histograma de movilidad para un ensamble de $2 \times 10^{3}$ trayectorias sampleadas de acuerdo con $p_{C}\left(\psi_{0}, \psi_{0}^{*}\right)$ para cuatro valores diferentes de $U / J$. El centro de las distribuciones se corre monótonamente hacia valores menores de la movilidad a medida que aumentamos $U / J$, pero la dispersión alrededor del centro de la distribución disminuye para acoplamientos grandes. En particular, para acoplamientos lo suficientemente altos todas las trayectorias tienen una movilidad muy pequeña con una dispersión pequeña, mostrando que todas las trayectorias están «congeladas», al menos en escalas de tiempo menores o iguales 
Capítulo 5. Estados pretermalizados y comportamiento tipo vidrio en sistemas casi integrables en una dimensión

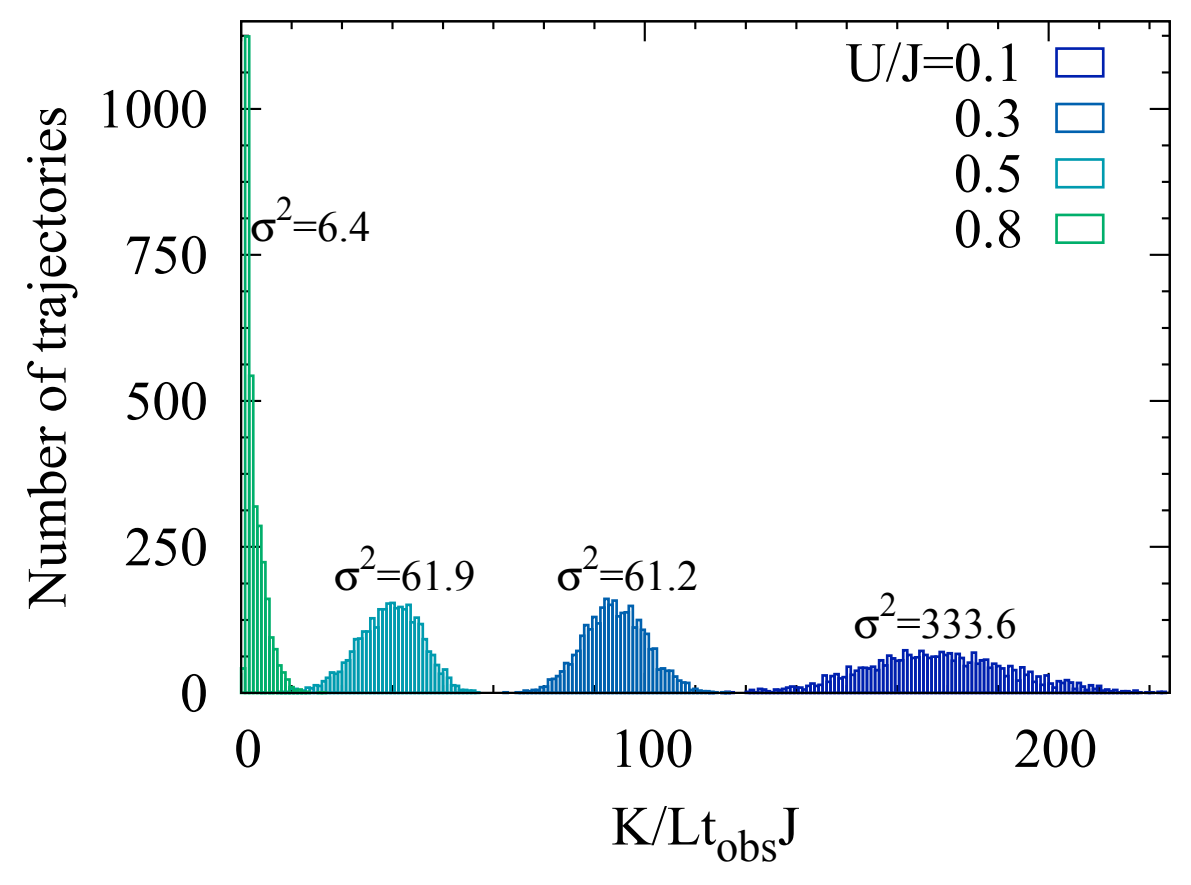

Figura 5.12: Histograma de la movilidad $K[\psi(t)]$ para ensambles de $2 \times 10^{3}$ trayectorias muestreadas de acuerdo con la función de Wigner del estado coherente $p_{C}\left(\psi_{0}, \psi_{0}^{*}\right)$ para cuatro valores diferentes de $U / J$. 
que $t_{\text {obs. }}$. Sin embargo, hay una zona de acoplamientos intermedios $0,3 \lesssim U / J \lesssim 0,6$, que se corresponde con el régimen intermedio descripto al analizar la relajación de la energía cinética, para la cual la dispersión prácticamente no cambia. Esto confirma que la aparición de trayectorias inactivas, no ergódicas, está relacionada con la existencia de estados térmices metaestables no térmicos en la dinámica cuántica. Además, dado que el corrimiento del centro de los histogramas es continuo podemos esperar que para acoplamientos intermedios haya coexistencia de trayectorias activas e inactivas, en cuyo caso la movilidad de las trayectorias debería depender de los detalles de la condición inicial. Este resulta ser el caso. Esta observación puede ser formulada en el lenguaje de una transición de fase dinámica [49, 50, 62, 32, 48] que tiene lugar en el ensamble de trayectorias clásicas.

Una transición de fase dinámica puede ser definida en completa analogía con una transición de fase en equilibrio [62]. En la descripción de mecánica estadística de una transición de fase de equilibrio generalmente se considera un ensamble de microestados de un dado sistema y una cierta cantidad extensiva que caracteriza cada uno de los microestados, como, por ejemplo, la energía de cada configuración en un momdelo de Ising clásico. Acto seguido, se introduce una disstrbución de probabilidad canónica que confiere diferentes pesos a cada microestado dependiendo del valor que toma la cantidad extensiva en cada caso. Si la cantidad extensiva elegida es la energía, entonces la distribución de probabilidad es el familiar $\frac{1}{Z} e^{-\beta H}$, en donde $H$ es el Hamiltoniano, $\beta$, la cantidad intensiva conjugada a la energía, es la temperatura inversa y $Z$ es la función de partición. Para analizar la transición de fase se considera el promedio de un dado parámetro de orden, como la magnetización en el caso del modelo de Ising, entre todos los microestados, pesados con la distribuciónd e probabilidad canónica. La transición de fase se manifiesta entonces como un cambio abrupto en el valor del promedio del parámetro de roden al variar el parámetro intensivo, $\beta$ en el caso del modelo de Ising. Hablamos entonces de una transición de fase dirigida por la temperatura. Para definir una transición de fase dinámica hacemos mecánica estadística con las trayectorias del sistema, y las consideramos como los «microestados». En nuestro caso, elegimos la movilidad $K[\psi(t)]$ como la cantidad extensiva en el tiempo que utilizaremos para caracterizar cada trayectoria. Construímos entonces una distribución de probabilidad canónica sobre el ensamble de trayectorias acoplando la cantidad extensiva en el tiempo $K[\psi(t)]$ con una cantidad intensiva $s$, que es el análogo de la temperatura en el ejemplo discutido antes. Esta distribución será:

$$
P_{s}[\psi(t)]=\frac{1}{Z_{s}} P_{0}[\psi(t)] e^{-s K[\psi(t)]},
$$

en donde $P_{0}[\psi(t)]$ es la distribución de probabilidad a $s=0$ que tomamos como la función de Wigner del estado coherente $p_{C}\left(\psi_{0}, \psi_{0}^{*}\right)$, tal y como fue discutido antes y $Z_{s}$ es la función 


\section{Capítulo 5. Estados pretermalizados y comportamiento tipo vidrio en sistemas casi integrables en una dimensión}

de partición

$$
Z_{s}=\sum_{\psi(t)} P_{s}[\psi(t)]
$$

Calcularemos entonces valores de expectación en el ensamble de trayectorias sumando sobre todas las trayectorias pesadas con $P_{s}[\psi(t)]$ de la siguiente manera

$$
\Omega_{s}=\langle\Omega[\psi(t)]\rangle_{s}=\frac{1}{Z_{s}} \sum_{\psi(t)} P_{s}[\psi(t)] \Omega[\psi(t)],
$$

en donde $\Omega[\psi(t)]$ es una funcional de la trayectoria. Esta manera de promediar le da diferente peso a las trayectorias móviles o inmóviles dependiendo del valor des: para $s$ grande se le da más peso relativo a las trayectorias inactivas y viceversa.

Para analizar la transición de fase elegimos a la movilidad $K[\psi(t)]$ como parámetro de orden (en el problema que estamos atacando la cantidad extensiva en el tiempo y el parámetro de orden coinciden). En la Fig. 5.13 mostramos el promedio del parámetro de orden $K_{s}$ como función de $s$ para $U / J=0,53$, un valor de $U / J$ para el cual hemos observado la coexistencia de trayectorias activas e inactivas. El comportameinto de $K_{s}$ es análogo al comportamiento del parámetro de orden en una transición de fase de equilibrio a volumen finito: exhibe un marcado escalón entre dos valores bien definidos correspondientes a las fases en el ensamble (fases activa e inactiva) y, además, al incrementar $t_{\mathrm{obs}}$ (el análogo del volumen en equilibrio) el escalón en $K_{s}$ se vuelve más y más abrupto. Esto puede apreciarse más claramente estudiando la susceptibilidad:

$$
\chi_{s}=-\frac{\partial K_{s}}{\partial s}=\left\langle\left(K[\psi(t)]-K_{s}\right)^{2}\right\rangle_{s},
$$

que exhibe un pico que crece al incrementar $t_{\text {obs. }}$. Un escaleo similar puede observarse al variar $L$. El valor crítico está localizado alrededor de cero, $s^{*}=0$. Esta transición de fase dinámica es muy similar a aquella descubierta en principio para modelos de vidrios con ligaduras cinéticas [49], luego para modelos atómicos de vidrios [62] y para sistemas cuánticos [48]. La diferencia principal que encontrasmos con respecto a estos trabajos es que la presente transición dinámica no parece ser de primer orden. En una transición de fase de primer orden (dinámica o estática) la distribución del parámetro de orden en la coexistencia es bimodal debido a efectos de tensión superficial entre los dominios coexistentes de las diferentes fases. En el caso analizado en este trabajo el parámetro de orden en coexistencia ( $s \simeq 0$ para $U / J=0,5$ ) es unimodal, lo cual puede inferirse considerando la Fig. 5.12, lo cual es compatible con una transición de fase continua.

Para caracterizar mejor las propiedades física de las fases discutiremos una medida de ergodi- 

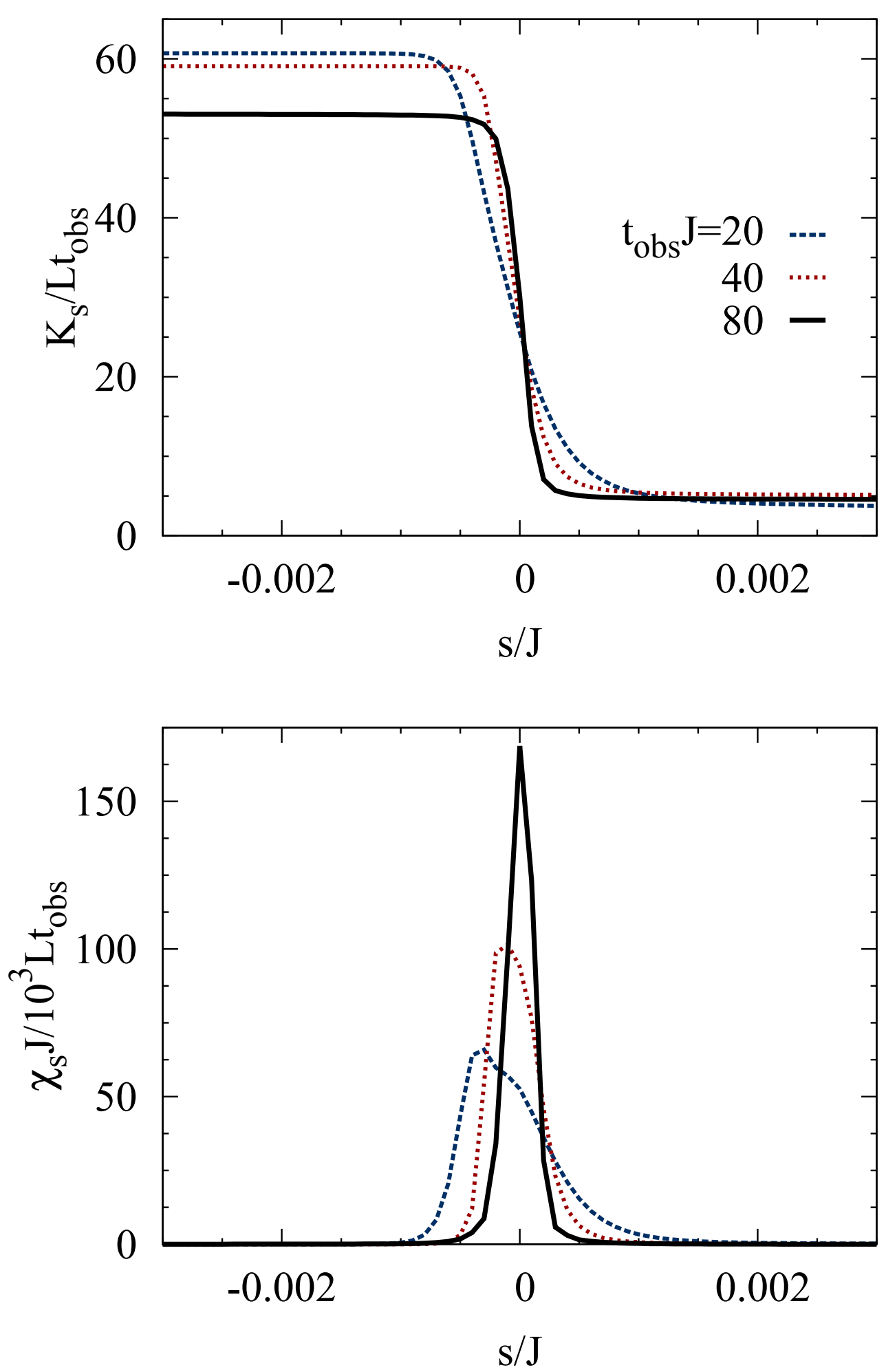

Figura 5.13: Transición de fase dinámica en el espacio de trayectorias. Panel superior: Promedio del parámetro de orden $K_{s}$ como función del campo intensivo $s$ para un ensamble de $10^{3}$ trayectorias muestreadas de acuerdo a la función de Wigner del estado coherente para $U / J=0,53$. Panel inferior: Susceptibilidad dinámica $\chi_{s}$. 
Capítulo 5. Estados pretermalizados y comportamiento tipo vidrio en sistemas casi integrables en una dimensión

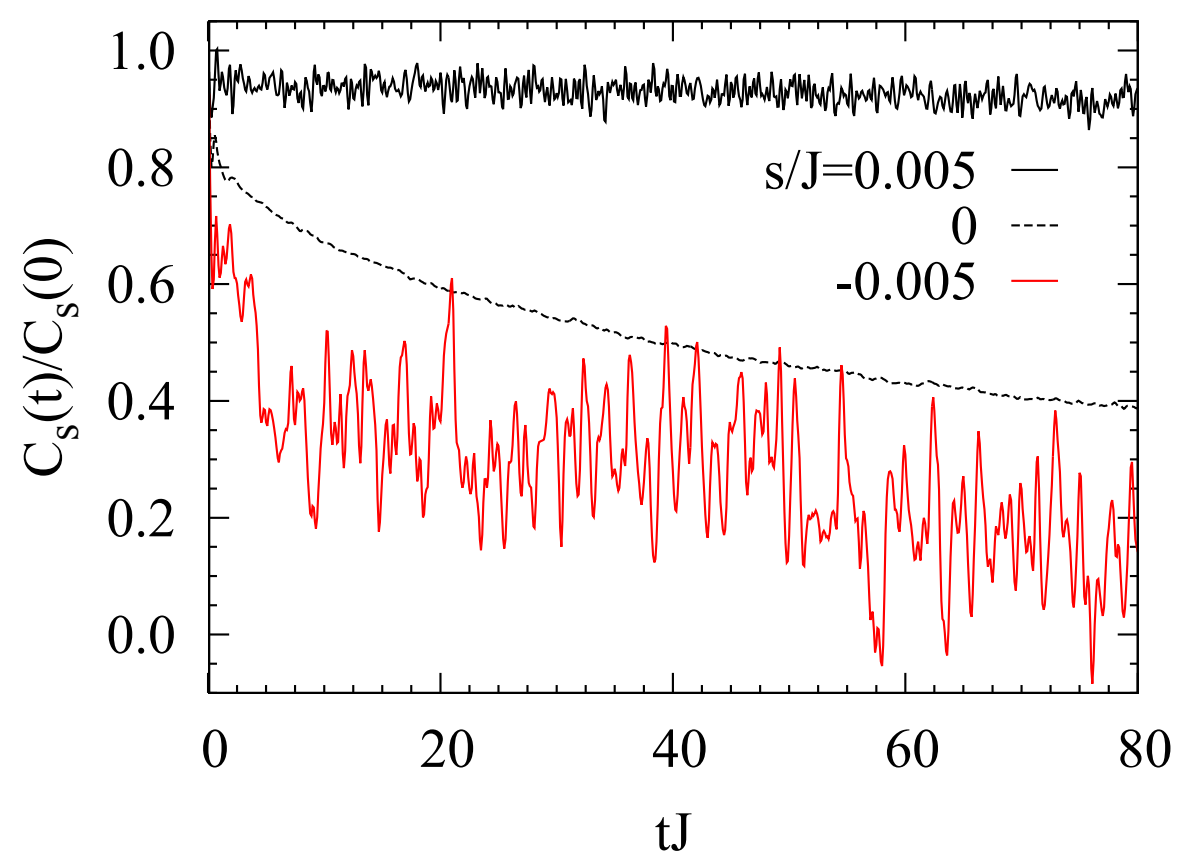

Figura 5.14: Función de correlación $C_{s}(t)$ como función del tiempo para $U / J=0,53$ en la fase activa $(s<0)$ e inactiva $(s>0)$. También mostramos, para comparar, la función de correlación en coexistencia $(s=0)$. 
cidad. Definimos la función de correlación de solapamiento como,

$$
C_{s}(t)=\sum_{j=1}^{L}\left\langle\left(n_{j}(t)-N\right)\left(n_{j}(0)-N\right)\right\rangle_{s}
$$

$C_{s}(t)$ cuantifica el solapamiento entre la configuración a tiempo $t$ y la configuración inicial. Su apartamiento de cero a tiempos largos es una medida de no ergodicidad. En la Fig. 5.14 mostramos la función de correlación $C_{s}(t)$ para la fase activa (ergódica) e inactiva (no ergódica). En la fase activa $(s<0)$ la función de correlación relaja rápidamente a un valor pequeño, mientras que para la fase inactiva $(s>0)$ las trayectorias se quedan atrapadas en un solo estado durante todo el tiempo de observación.

En modelos clásicos de vidrios esta transición de fase dinámica representa una de las teorías más importantes para dar cuenta de un extraordinaria y distintiva característica de los materiales vidriosos: la heterogeneidad dinámica [32]. En contraste con los fluidos normales, la relajación de los vidrios es inhomogénea en el espacio: regiones rápidas y lentas están conglomeradas en el espacio. Hemos descubierto que las trayectorias clásicas individuales (que corresponden a soluciones aislada de las ecuaciones de Gross-Pitaevskii) exhiben el fenómeno de heterogeneidad dinámica. Definimos una medida local de movilidad $Q_{i}(t)$ como

$$
Q_{i}(t)=\left|n_{i}(t)-n_{i}(0)\right|,
$$

que mide en qué medida la densidad de un sitio difiere del valor inicial. En la Fig. 5.15 mostramos los gráficos de $Q_{i}(t)$ para dos trayectorias representativas correspondientes a $U / J=0,1$ y 0,6. Para $U / J=0,1$ (el régimen de acoplamientos débiles) la dinámica del sistema, tal y como es capturada por el indicador $Q_{i}(t)$, es bastante homogénea. Para $U / J=0,6$ (entrando en el régimen de acoplamientos fuertes), los sitios con movilidad local alta y movilidad local baja están agrupados en el espacio-tiempo. Esto es un sello distintivo de la heterogeneidad dinámica. Es relamente notable que la simple ecuación de Gross-Pitaevskii sea capaz de generar una dinámica tan complicada.

La relación entre la transición de fase dinámica y la heterogeneidad dinámica puede explicarse usando un razonamiento simple: la transición de fase dinámica implica que para valores intermedios de $U / J$ diferentes condiciones iniciales pueden gatillar una dinámica lenta o rápida, dependiendo sólo de los detalles de la condición inicial; si consideramos un sistema lo suficientemente grande con interacciones de corto alcance, regiones distantes no van a estar correlacionadas y si una región tiene una condión inicial rápida y otra una lenta la heterogeneidad dinámica ocurrirá. Además, con el mismo argumento, durante la evolución dinámica las regiones lentas pueden convertirse en rápidas y viceversa. Al contrario de lo que se observa en [106, 105], la dinámica cuántica no muestra heterogeneidad dinámica en nuestro caso. El promedio necesario para obtener la dinámica cuántica a partir de las trayectorias individuales clásicas borra todo patrón dinámico heterogéneo que dependa de las condiciones iniciales, lo cual está asociado al hecho de que el perfil de densidad tiene una 

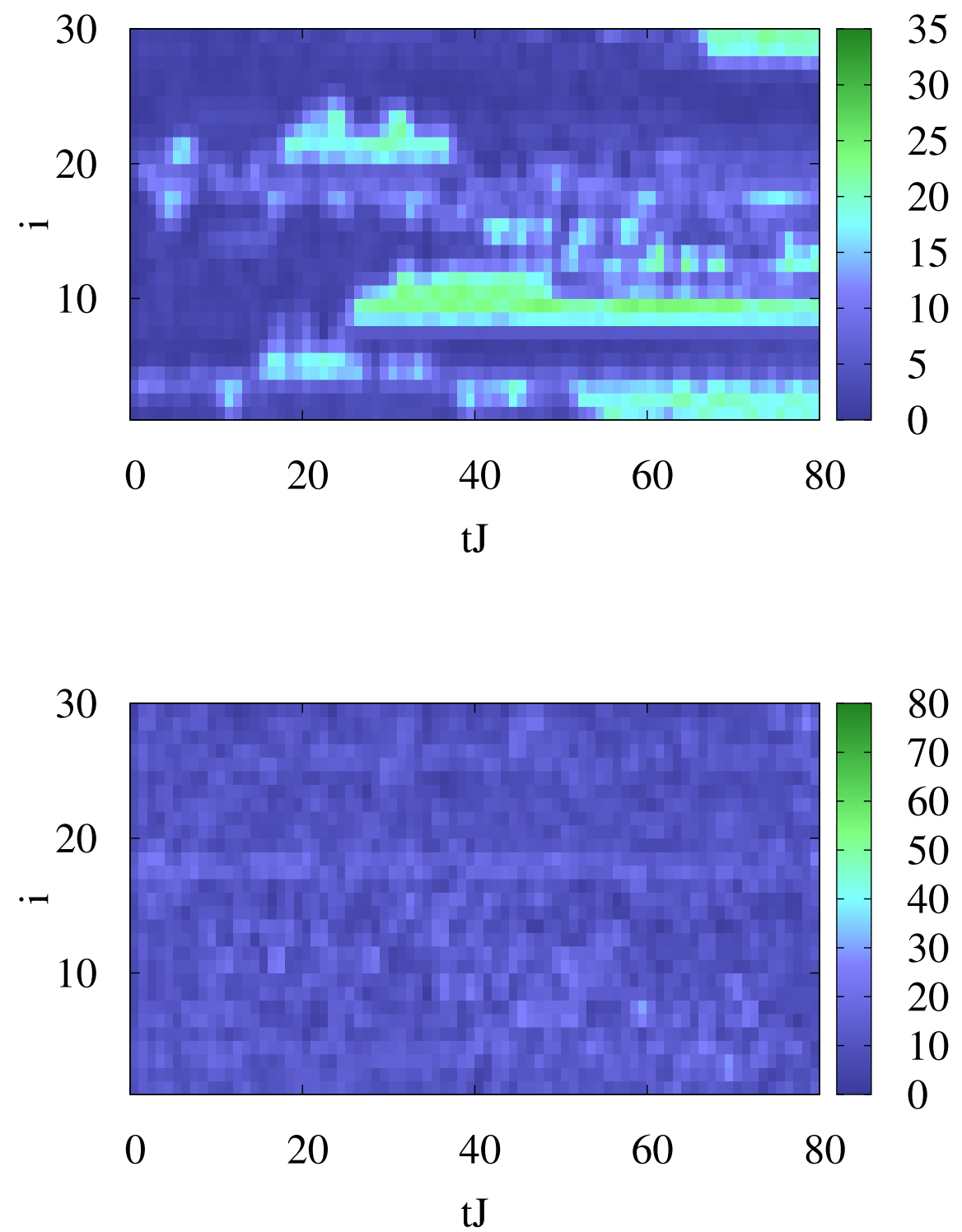

Figura 5.15: Gráfico de color falso de la movilidad local $Q_{i}(t)$ para dos trayectorias representativas correspondientes a $U / J=0,6$ (panel superior) y $U / J=0,1$ (panel inferior). 
escala de relajación bien definida como hemos visto antes.

\subsection{Estados pretermalizados lejos de un punto integrable}

En las primeras discusiones acerca de la existencia de estados pretermalizados que aparecieron en la literatura, en Referencias como [78] y [90], se afirma que las cercanías de un punto integrable es uno de los factores determinantes para la aparición de este tipo de fenomenología. Sin embargo, muy recientemente se han encontrado estados pretermalizados con vidas medias muy largas en sistemas en $D>1$ en regiones de parámetros que no están cerca de los puntos integrables triviales (modelos no interactuantes), los únicos conocidos en $D>1$. Podemos citar dos ejemplos: (i) la Ref. [109], en la que se encuentran plateaus extremadamente largos en funciones de correlación de dos tiempos para un modelo de Ising con una perturbación periódica en el tiempo, y (ii) el preprint [1] en donde se analiza un quench en el modelo de Heisenber isotrópico en $D=3$ y se encuentra que existen estados pretermalizados con vidas medias muy largas para ciertas configuraciones iniciales.

En estos modelos, la escasez de canales de relajación no está asociada a la cercanía de ningún punto integrable. De hecho, en la Ref. [109] se mostró que aparecen plateaus en regiones de parámetros para los cuales el espectro del Hamiltoniano de evolución muestra claras señales de caoticidad cuántica, una de las marcas de la no integrabilidad. Estos resultados refuerzan la perspectiva presentada en esta tesis de que la integrabilidad no es la clave que explica la emergencia de etados pretermalizados, sino que lo que cuenta es la escasez de canales de relajación. Esta escasez parece estar correlacionada en $D=1$ con la proximidad a un punto integrable pero en principio puede deberse a otras restricciones en la dinámica. Como fue señalado más arriba, nuestros resultados indican que un factor decisivo para que exista escasez de canales de relajación y para la aparición de estados pretermalizados es que el quench sea débil, es decir, que la energía del sistema esté muy cerca de la energía del estado fundamental del Hamiltoniano de evolución, lo cual se verifica en las situaciones investigadas en las mencionadas referencias. 



\section{Conclusiones y perspectivas}

Esta tesis pretende ser un aporte al estudio de la dinámica de sistemas cuánticos aislados fuera de equilibrio. Tal como ha sido descripto en la introducción y desarrollado en los Capítulos que conforman el cuerpo de la exposición, este tópico de investigación es fundamental para comprender las bases de la mecánica estadística. También tiene relevancia experimental debido a los experimentos recientes que se han realizado con átomos fríos cargados en redes ópticas.

Más allá de que hemos obtenido nuestros resultados atacando modelos diferentes con las técnicas apropiadas para cada caso, creemos que contamos con una imagen de aplicación general acerca de la dinámica de no equilibrio que se desarrolla después de un quench. Para quenches débiles, en los que la energía inyectada al sistema es pequeña comparada con las escalas de energía características del sistema, y si los canales de relajación (diferentes del dephasing) del sistema son escasos (como lo que se encuentra cerca de un punto integrable en $D=1$ ), surgen estados metaestables que se interponen entre el estado inicial y el estado final del sistema. Tales estados metaestables surgen como consecuencia del dephasing entre ciertos modos libres que describen la dinámica a tiempos cortos. Hemos sido capaces de identificar explícitamente estos modos cuasilibres en sistemas de fermiones unidimensionales (Capítulo 2 y 5) y bidimensionales (Capítulo 3). Estos estados corresponden con el estado final de la dinámica de un modelo cuadrático efectivo que describe la evolución de tiempos cortos y, por lo tanto, admiten una descripción en términos de un ensamble de Gibbs generalizado que tenga en cuenta todas las constantes de movimiento triviales del modelo cuadrático (Capítulo 3). La vida media de estos estados depende fuertemente de la disponibilidad de canales de relajación inelásticos en el sistema. Si estos canales están ausentes o si son escasos (tal como es el caso cerca de un punto integrable a bajas energías) su vida media puede ser muy larga comparada con los tiempos de relajación del mismo sistema en otras regiones de parámetros (Capítulo 5). Esto puede obstaculizar completamente la observación del equilibrio térmico final del sistema en simulaciones numéricas o en experimentos. La existencia de estados metaestables que surgen a tiempos cortos y que poseen vidas medias enormes plantea una analogía con la física de los vidrios. En el Capítulo 5 hemos explorado esa analogía. 
Al presente, creemos que hay dos cuestiones muy fundamentales y concretas que es necesario resolver para validar/enmendar y/o completar este panorama. Primero creemos que sería interesante investigar la conexión entre la dinámica (aparición de estados metaestables y su vida media) y la relación entre la energía del estado inicial y la energía del estado fundamental y otras características del espectro del Hamiltoniano de evolución. Esta cuestión surge de considerar las discusiones alrededor del sistema de fermiones estudiado en el Capítulo 5. Preguntas relevantes serían: ¿Cuál es la relación entre la diferencia de energía del estado inicial y del estado fundamental del Hamiltoniano de evolución $\Delta E=\operatorname{Tr}[\rho(0) H]-\langle\Psi|H| \Psi\rangle$ con la vida media de los estados pretermalizados? ¿Qué sucede si existe un gap en el espectro de $H$ ? ¿Depende fuertemente la dinámica de si la energía del estado inicial cae en el gap o fuera de éste? Para contestar esta preguntas habría que calcular el espectro de $H$ usando técnicas numéricas en equilibrio y contrastar con la dinámica fuera de equilibrio obtenida, por ejemplo, usando las ecuaciones de movimiento deducidas en el Capítulo 4.

Otra cuestión sumamente importante es la del estado inicial. En todos los problemas atacados en esta tesis, salvo en el sistema bosónico tratado en el Capítulo 5, hemos considerado estados iniciales no correlacionados. Es necesario saber si el panorama descripto más arriba sobrevive al considerar estados iniciales con interacciones. Los resultados obtenidos para el modelo de Hubbard bosónico en el Capítulo 5 parecen respaldar la respuesta afirmativa, pero es necesario realizar más investigaciones. Por ejemplo, en el formalismo del operador proyección, la introducción de interacciones en el estado inicial lleva a la no nulidad del término de ruido microscópico $P(t) L(t) G(t, 0) Q(0) \tilde{\rho}(0)$. Encontrar aproximaciones para este término es posible [12], pero es una empresa en sí misma. Otra alternativa es usar métodos numéricos estandar que permitan estudiar tiempos largos, como diagonalización exacta aunque esto nos restringiría a sistemas pequeños, lejos del límite termodinámico.

Finalmente, también sería muy importante contrastar los resultados obtenidos a través de las ecuaciones de evolución deducidas en el Capítulo 4 con técnicas numéricas estándar, algo en lo que estamos trabajando actualmente. Por ejemplo, comparar con t-DMRG para sistemas grandes y tiempos cortos y diagonalización exacta para sistemas pequeños y tiempos arbitrarios.

Esperamos que los resultados y perspectivas presentados en esta tesis sirvan para motivar y dirigir nuevas investigaciones y aporten al conocimiento general en el campo de la dinámica de no equilibrio de sistemas cuánticos aislados. 


\section{A Apéndice}

\section{A.1. Propiedades del operador proyección $P(t)$}

En este Ápéndice probaremos las propiedades en la Ec. 4.9.

1. $P(t) \tilde{\rho}(t)=\tilde{\sigma}(t)$

$$
\begin{aligned}
P(t) \tilde{\rho}(t) & =\left(\sigma(t)-\sum_{\boldsymbol{k}} \frac{\delta \sigma(t)}{\delta\langle n(\boldsymbol{k})\rangle_{t}}\langle n(\boldsymbol{k})\rangle_{t}\right) \operatorname{Tr}[\tilde{\rho}(t)]+\sum_{\boldsymbol{k}} \frac{\delta \sigma(t)}{\delta\langle n(\boldsymbol{k})\rangle_{t}} \operatorname{Tr}[n(\boldsymbol{k}) \tilde{\rho}(t)] \\
& =\left(\sigma(t)-\sum_{\boldsymbol{k}} \frac{\delta \sigma(t)}{\delta\langle n(\boldsymbol{k})\rangle_{t}}\langle n(\boldsymbol{k})\rangle_{t}\right)+\sum_{\boldsymbol{k}} \frac{\delta \sigma(t)}{\delta\langle n(\boldsymbol{k})\rangle_{t}}\langle n(\boldsymbol{k})\rangle_{t} \\
& =\tilde{\sigma}(t) .
\end{aligned}
$$

2. $P(t) \partial_{t} \tilde{\rho}(t)=\partial_{t} \tilde{\sigma}(t)$

$$
\begin{aligned}
P(t) \partial_{t} \tilde{\rho}(t) & =\left(\sigma(t)-\sum_{\boldsymbol{k}} \frac{\delta \sigma(t)}{\delta\langle n(\boldsymbol{k})\rangle_{t}}\langle n(\boldsymbol{k})\rangle_{t}\right) \partial_{t} \operatorname{Tr}[\tilde{\rho}(t)]+\sum_{\boldsymbol{k}} \frac{\delta \sigma(t)}{\delta\langle n(\boldsymbol{k})\rangle_{t}} \partial_{t} \operatorname{Tr}[n(\boldsymbol{k}) \tilde{\rho}(t)] \\
& =\sum_{\boldsymbol{k}} \frac{\delta \sigma(t)}{\delta\langle n(\boldsymbol{k})\rangle_{t}} \partial_{t}\langle n(\boldsymbol{k})\rangle_{t} \\
& =\partial_{t} \tilde{\sigma}(t) .
\end{aligned}
$$


3. $\operatorname{Tr}[n(\boldsymbol{k}) P(t) O]=\operatorname{Tr}[n(\boldsymbol{k}) O]$

$$
\begin{aligned}
\operatorname{Tr}[n(\boldsymbol{k}) P(t) O]= & \left(\operatorname{Tr}[n(\boldsymbol{k}) \sigma(t)]-\sum_{\boldsymbol{k}^{\prime}} \frac{\delta \operatorname{Tr}[n(\boldsymbol{k}) \sigma(t)]}{\delta\left\langle n\left(\boldsymbol{k}^{\prime}\right)\right\rangle_{t}}\left\langle n\left(\boldsymbol{k}^{\prime}\right)\right\rangle_{t}\right) \operatorname{Tr}[O]+ \\
& \sum_{\boldsymbol{k}^{\prime}} \frac{\delta \operatorname{Tr}\left[n\left(\boldsymbol{k}^{\prime}\right) \sigma(t)\right]}{\delta\left\langle n\left(\boldsymbol{k}^{\prime}\right)\right\rangle_{t}} \operatorname{Tr}\left[n\left(\boldsymbol{k}^{\prime}\right) O\right] \\
= & \left(\operatorname{Tr}[n(\boldsymbol{k}) \sigma(t)]-\sum_{\boldsymbol{k}^{\prime}} \delta_{\boldsymbol{k}, \boldsymbol{k}^{\prime}}\left\langle n\left(\boldsymbol{k}^{\prime}\right)\right\rangle_{t}\right) \operatorname{Tr}[O]+\sum_{\boldsymbol{k}^{\prime}} \delta_{\boldsymbol{k}, \boldsymbol{k}^{\prime}} \operatorname{Tr}\left[n\left(\boldsymbol{k}^{\prime}\right) O\right] \\
= & \operatorname{Tr}[n(\boldsymbol{k}) O] .
\end{aligned}
$$

4. $P(t) P\left(t^{\prime}\right) O=P(t) O$

$$
\begin{aligned}
P(t) P\left(t^{\prime}\right) O= & \left(\sigma(t)-\sum_{\boldsymbol{k}^{\prime \prime}} \frac{\delta \sigma(t)}{\delta\left\langle n\left(\boldsymbol{k}^{\prime \prime}\right)\right\rangle_{t}}\left\langle n\left(\boldsymbol{k}^{\prime \prime}\right)\right\rangle_{t}\right) \times \\
& \left(\operatorname{Tr}\left[\sigma\left(t^{\prime}\right)\right]-\sum_{\boldsymbol{k}^{\prime}} \frac{\delta \operatorname{Tr}\left[\sigma\left(t^{\prime}\right)\right]}{\delta\left\langle n\left(\boldsymbol{k}^{\prime}\right)\right\rangle_{t^{\prime}}}\left\langle n\left(\boldsymbol{k}^{\prime}\right)\right\rangle_{t^{\prime}}\right) \operatorname{Tr}[O]+ \\
& \sum_{\boldsymbol{k}^{\prime}, \boldsymbol{k}^{\prime \prime}} \frac{\delta \operatorname{Tr}\left[n\left(\boldsymbol{k}^{\prime \prime}\right) \sigma\left(t^{\prime}\right)\right]}{\delta\left\langle n\left(\boldsymbol{k}^{\prime}\right)\right\rangle_{t^{\prime}}} \operatorname{Tr}\left[n\left(\boldsymbol{k}^{\prime}\right) O\right] \frac{\delta \sigma(t)}{\delta\left\langle n\left(\boldsymbol{k}^{\prime \prime}\right)\right\rangle_{t}} \\
= & \left(\sigma(t)-\sum_{\boldsymbol{k}^{\prime \prime}} \frac{\delta \sigma(t)}{\delta\left\langle n\left(\boldsymbol{k}^{\prime \prime}\right)\right\rangle_{t}}\left\langle n\left(\boldsymbol{k}^{\prime \prime}\right)\right\rangle_{t}\right) \operatorname{Tr}[O]+\sum_{\boldsymbol{k}^{\prime \prime}} \frac{\delta \sigma(t)}{\delta\left\langle n\left(\boldsymbol{k}^{\prime \prime}\right)\right\rangle_{t}} \operatorname{Tr}\left[n\left(\boldsymbol{k}^{\prime \prime}\right) O\right] \\
= & P(t) O .
\end{aligned}
$$

5. $P(t) L(t) P(s) O=0$ para sistemas que conservan momento total,

$$
\begin{aligned}
P(t) L(t) P(s) O= & \left(\sigma(t)-\sum_{\boldsymbol{k}^{\prime \prime}} \frac{\delta \sigma(t)}{\delta\left\langle n\left(\boldsymbol{k}^{\prime \prime}\right)\right\rangle_{t}}\left\langle n\left(\boldsymbol{k}^{\prime \prime}\right)\right\rangle_{t}\right) \times \\
& {\left[\left(\operatorname{Tr}\left\{\left[H_{1}(t), \sigma(s)\right]\right\}-\sum_{\boldsymbol{k}^{\prime}} \frac{\delta \operatorname{Tr}\left\{\left[H_{1}(t), \sigma(s)\right]\right\}}{\delta\left\langle n\left(\boldsymbol{k}^{\prime}\right)\right\rangle_{s}}\left\langle n\left(\boldsymbol{k}^{\prime}\right)\right\rangle_{s}\right) \operatorname{Tr}[O]+\right.} \\
& \left.\sum_{\boldsymbol{k}^{\prime}} \frac{\delta \operatorname{Tr}\left\{\left[H_{1}(t), \sigma(s)\right]\right\}}{\delta\left\langle n\left(\boldsymbol{k}^{\prime}\right)\right\rangle_{s}} \operatorname{Tr}\left[n\left(\boldsymbol{k}^{\prime}\right) O\right]\right]+ \\
& \sum_{\boldsymbol{k}^{\prime \prime}} \frac{\delta \sigma(t)}{\delta\left\langle n\left(\boldsymbol{k}^{\prime \prime}\right)\right\rangle_{t}}\left[\left(\operatorname{Tr}\left\{n\left(\boldsymbol{k}^{\prime \prime}\right)\left[H_{1}(t), \sigma(s)\right]\right\}-\sum_{\boldsymbol{k}^{\prime}} \frac{\delta \operatorname{Tr}\left\{n\left(\boldsymbol{k}^{\prime \prime}\right)\left[H_{1}(t), \sigma(s)\right]\right\}}{\delta\left\langle n\left(\boldsymbol{k}^{\prime}\right)\right\rangle_{s}}\left\langle n\left(\boldsymbol{k}^{\prime}\right)\right\rangle_{s}\right) \operatorname{Tr}[O]+\right. \\
& \left.\sum_{\boldsymbol{k}^{\prime}} \frac{\delta \operatorname{Tr}\left\{n\left(\boldsymbol{k}^{\prime \prime}\right)\left[H_{1}(t), \sigma(s)\right]\right\}}{\delta\left\langle n\left(\boldsymbol{k}^{\prime}\right)\right\rangle_{s}} \operatorname{Tr}\left[n\left(\boldsymbol{k}^{\prime}\right) O\right]\right]=0 .
\end{aligned}
$$

Dado que $\operatorname{Tr}\left\{\left[H_{1}(t), \sigma(s)\right]\right\}=0$ (por la propiedad cíclica de la traza) y que $\operatorname{Tr}\left\{n\left(\boldsymbol{k}^{\prime \prime}\right)\left[H_{1}(t), \sigma(s)\right]\right\}=$ $\operatorname{Tr}\left\{\left[H_{1}(t), n\left(\boldsymbol{k}^{\prime \prime}\right)\right] \sigma(s)\right\}=0$, lo cual puede ser verificado por un cálculo directo:

$$
\left[H_{1}(t), n\left(\boldsymbol{k}^{\prime \prime}\right)\right]=\sum_{\boldsymbol{k}_{1}, \boldsymbol{k}_{2}, \boldsymbol{k}_{3}, \boldsymbol{k}_{4}} W\left(\boldsymbol{k}_{1}, \boldsymbol{k}_{2}, \boldsymbol{k}_{3}, \boldsymbol{k}_{\mathbf{4}} ; \boldsymbol{k}^{\prime \prime}, \boldsymbol{t}\right) c^{\dagger}\left(\boldsymbol{k}_{1}\right) c^{\dagger}\left(\boldsymbol{k}_{2}\right) c\left(\boldsymbol{k}_{3}\right) c\left(\boldsymbol{k}_{4}\right),
$$


en donde $W\left(\boldsymbol{k}_{1}, \boldsymbol{k}_{\mathbf{2}}, \boldsymbol{k}_{\mathbf{3}}, \boldsymbol{k}_{\mathbf{4}} ; \boldsymbol{k}^{\prime \prime}, \boldsymbol{t}\right)=V\left(\boldsymbol{k}_{1}, \boldsymbol{k}_{\mathbf{2}}, \boldsymbol{k}_{\mathbf{3}}, \boldsymbol{k}_{\mathbf{4}}\right)\left(\delta_{\boldsymbol{k}_{1}, \boldsymbol{k}^{\prime \prime}}+\delta_{\boldsymbol{k}_{2}, \boldsymbol{k}^{\prime \prime}}-\delta_{\boldsymbol{k}_{3}, \boldsymbol{k}^{\prime \prime}}-\delta_{\boldsymbol{k}_{4}, \boldsymbol{k}^{\prime \prime}}\right) \exp \left[\operatorname{it}\left(\epsilon\left(\boldsymbol{k}_{1}\right)+\right.\right.$ $\left.\left.\epsilon\left(\boldsymbol{k}_{2}\right)-\epsilon\left(\boldsymbol{k}_{3}\right)-\epsilon\left(\boldsymbol{k}_{4}\right)\right)\right]$. Por otro lado, usando la regla de Wick obtenemos que

$\operatorname{Tr}\left\{c^{\dagger}\left(\boldsymbol{k}_{1}\right) c^{\dagger}\left(\boldsymbol{k}_{2}\right) c\left(\boldsymbol{k}_{3}\right) c\left(\boldsymbol{k}_{4}\right) \sigma(s)\right\}=\delta_{\boldsymbol{k}_{1}, \boldsymbol{k}_{4}} \delta_{\boldsymbol{k}_{2}, \boldsymbol{k}_{3}}\left\langle n\left(\boldsymbol{k}_{1}\right)\right\rangle_{s}\left\langle n\left(\boldsymbol{k}_{2}\right)\right\rangle_{s}-\delta_{\boldsymbol{k}_{2}, \boldsymbol{k}_{4}} \delta_{\boldsymbol{k}_{1}, \boldsymbol{k}_{3}}\left\langle n\left(\boldsymbol{k}_{1}\right)\right\rangle_{s}\left\langle n\left(\boldsymbol{k}_{2}\right)\right\rangle_{s}$.

Ninguno de los términos sobrevive dada la combinación de las dos $\delta$ con las $\delta$ presentes en $W\left(\boldsymbol{k}_{1}, \boldsymbol{k}_{2}, \boldsymbol{k}_{\mathbf{3}}, \boldsymbol{k}_{\mathbf{4}} ; \boldsymbol{k}^{\prime \prime}, \boldsymbol{t}\right)$. Por ejemplo, para el primer término tenemos que $\delta_{\boldsymbol{k}_{1}, \boldsymbol{k}_{4}} \delta_{\boldsymbol{k}_{2}, \boldsymbol{k}_{3}}\left(\delta_{\boldsymbol{k}_{1}, \boldsymbol{k}^{\prime \prime}}+\delta_{\boldsymbol{k}_{2}, \boldsymbol{k}^{\prime \prime}}-\delta_{\boldsymbol{k}_{3}, \boldsymbol{k}^{\prime \prime}}-\delta_{\boldsymbol{k}_{4}, \boldsymbol{k}^{\prime \prime}}\right)=\left(\delta_{\boldsymbol{k}_{1}, \boldsymbol{k}^{\prime \prime}}+\delta_{\boldsymbol{k}_{2}, \boldsymbol{k}^{\prime \prime}}-\delta_{\boldsymbol{k}_{1}, \boldsymbol{k}^{\prime \prime}}-\delta_{\boldsymbol{k}_{2}, \boldsymbol{k}^{\prime \prime}}\right)=0$.

\section{A.2. Exponenciales ordenadas}

En este Apéndice definiremos cuidadosamente las exponenciales ordenadas utilizadas en el texto y mostraremos como surgen naturalmente en el formalismo del operador proyección. Para más detalles pueden consultar las Refs. [45] y [77].

\section{A.2.1. Representación de interacción}

Aquí proveemos las definiciones precisas y propiedades de las exponenciales ordenadas que aparecen en el formalismo. Empezamos con $G(t, s)$, la exponencial ordenada que se utiliza para resolver la ecuación para la parte irrelevante de la matriz densidad (ver Ec. 4.15):

$$
\partial_{t} Q(t) \tilde{\rho}(t)=\alpha Q(t) L(t) P(t) \tilde{\rho}(t)+\alpha Q(t) L(t) Q(t) \tilde{\rho}(t) .
$$

Para resolverla multiplicamos por $G(s, t)$ por la izquierda:

$$
G(s, t) \partial_{t} Q(t) \tilde{\rho}(t)=\alpha G(s, t) Q(t) L(t) P(t) \tilde{\rho}(t)+\alpha G(s, t) Q(t) L(t) Q(t) \tilde{\rho}(t),
$$

e imponemos que

$$
\partial_{t} G(s, t)=-\alpha G(s, t) Q(t) L(t),
$$

de manera que $\alpha G(s, t) Q(t) L(t) Q(t) \tilde{\rho}(t)=-\partial_{t} G(s, t) Q(t) \tilde{\rho}(t)$. Luego completamos la derivada del producto en el miembro derecho de la Ec. A.3 y obtenemos

$$
\partial_{t} G(s, t) Q(t) \tilde{\rho}(t)=\alpha G(s, t) Q(t) L(t) P(t) \tilde{\rho}(t) .
$$

Integrando obtenemos:

$$
G(t, t) Q(t) \tilde{\rho}(t)-G(t, 0) Q(0) \tilde{\rho}(0)=\alpha \int_{0}^{t} d s G(s, t) Q(s) L(s) P(s) \tilde{\rho}(s) .
$$

Imponiendo la condición de contorno

$$
G(t, t)=I,
$$


obtenemos finalmente

$$
Q(t) \tilde{\rho}(t)=G(t, 0) Q(0) \tilde{\rho}(0)+\alpha \int_{0}^{t} d s G(s, t) Q(s) L(s) P(s) \tilde{\rho}(s) .
$$

En resumen, $G(t, s)$ viene definida por

$$
\begin{aligned}
\partial_{t} G(s, t) & =-\alpha G(s, t) Q(t) L(t), \\
G(s, s) & =I .
\end{aligned}
$$

La solución de esta ecuación puede encontrarse por iteración:

$$
G(s, t)=I+\sum_{n=1}^{\infty}(-\alpha)^{n} \int_{s}^{t} d t_{1} \int_{s}^{t_{1}} d t_{2} \ldots \int_{s}^{t_{n-1}} d t_{n} Q\left(t_{n}\right) L\left(t_{n}\right) Q\left(t_{n-1}\right) L\left(t_{n-1}\right) \ldots Q\left(t_{1}\right) L\left(t_{1}\right) .
$$

En símbolos:

$$
G(s, t)=T_{\rightarrow} \exp \left[-\alpha \int_{s}^{t} d s^{\prime} Q\left(s^{\prime}\right) L\left(s^{\prime}\right)\right],
$$

en donde $T_{\rightarrow}$ ordena los operadores de manera que el argumento temporal aumenta de izquierda a derecha (orden anticronológico).

\section{A.2.2. Representación de Heisenberg}

Deseamos resolver la Ec. (4.33):

$$
\partial_{t}\left[e^{i L t} \mathrm{Q}(t)\right]-e^{i L t} \mathrm{Q}(t) i L \mathrm{Q}(t)=e^{i L t} \mathrm{P}(t)[i L-\dot{\mathrm{P}}(t)] \mathrm{Q}(t),
$$

en donde $e^{i L t} \mathrm{Q}(t)$ es la incógnita y $e^{i L t} \mathrm{P}(t)[i L-\dot{\mathrm{P}}(t)] \mathrm{Q}(t)$ la inhomogeneidad. Para ello introducimos un factor integrante $\overline{\mathrm{G}}(s, t)$ que satisfaga

$$
\begin{aligned}
\partial_{t} \overline{\mathrm{G}}(s, t) & =-i L \mathrm{Q}(t) \overline{\mathrm{G}}(s, t), \\
\overline{\mathrm{G}}(s, s) & =I .
\end{aligned}
$$

Multiplicando la Ec. (A.13) por derecha por $\overline{\mathrm{G}}(s, t)$ y completando la derivada del producto obtenemos

$$
\partial_{t}\left[e^{i L t} \mathrm{Q}(t) \overline{\mathrm{G}}(s, t)\right]=e^{i L t} \mathrm{P}(t)[i L-\dot{\mathrm{P}}(t)] \mathrm{Q}(t)
$$


Integrando la última ecuación y usando la condición inicial de $\bar{G}(s, t)$ obtenemos la solución

$$
e^{i L t} \mathrm{Q}(t)=e^{i L s} \mathrm{Q}(s) \overline{\mathrm{G}}(t, s)+\int_{s}^{t} d u e^{i L u} \mathrm{P}(u)[i L-\dot{\mathrm{P}}(u)] \mathrm{Q}(u) \overline{\mathrm{G}}(t, u) .
$$

La forma iterativa de $\overline{\mathrm{G}}(s, t)$ es

$$
\overline{\mathrm{G}}(s, t)=I+\sum_{n=1}^{\infty} \int_{s}^{t} d t_{1} \int_{s}^{t_{1}} d t_{2} \ldots \int_{s}^{t_{n-1}} d t_{n}(-i L) \mathrm{Q}\left(t_{1}\right)(-i L) \mathrm{Q}\left(t_{2}\right) \ldots(-i L) \mathrm{Q}\left(t_{n}\right) .
$$

En símbolos:

$$
\overline{\mathrm{G}}(s, t)=T_{\leftarrow} \exp \left[\int_{s}^{t} d s^{\prime}(-i L) \mathrm{Q}\left(s^{\prime}\right)\right],
$$

en donde $T_{\leftarrow}$ ordena los operadores de manera que el argumento temporal aumenta de derecha a izquierda (orden cronológico). Para seguir la notación de la Ref. [54] en el texto principal se definió otra exponencial ordenada $\mathrm{G}(s, t)$ :

$$
\mathrm{G}(s, t)=T_{\leftarrow} \exp \left[\int_{s}^{t} d s^{\prime} i L Q\left(s^{\prime}\right)\right] .
$$

Es fácil ver que $\mathrm{G}(s, t)=\overline{\mathrm{G}}(t, s)$. 



\section{Bibliografía}

[1] Mehrtash Babadi, Eugene Demler, and Michael Knap. Far-from-equilibrium field theory of many-body quantum spin systems: Prethermalization and relaxation of spin spiral states in three dimensions. unpublished, 2015.

[2] P. Barmettler, D. Poletti, M. Cheneau, and C. Kollath. Propagation front of correlations in an interacting bose gas. Phys. Rev. A, 85:053625, 2012.

[3] T. Barthel and U. Schollwöck. Dephasing and the steady state in quantum many-particle systems. Phys. Rev. Lett., 100:100601, 2008. doi: 10.1103/PhysRevLett.100.100601.

[4] M. C. Bañuls, J. I. Cirac, , and M. B. Hastings. Strong and weak thermalization of infinite nonintegrable quantum systems. Phys. Rev. Lett., 106:050405, 2011.

[5] G. Baym and C. Pethick. Landau Fermi-Liquid Theory. Wiley, 1991.

[6] J. Berges. Introduction to nonequilibrium quantum field. AIP Conf. Proc., 739:3, 2004. doi: 10.1063/1.1843591. URL http://scitation.aip.org/content/aip/proceeding/aipcp/ $10.1063 / 1.1843591$.

[7] J. Berges and S. Borsányi. Range of validity of transport equations. Phys. Rev. D, 74: 045022, 2006.

[8] J. Berges, Sz. Borsányi, and C. Wetterich. Prethermalization. Phys. Rev. Lett., 93:142002, Sep 2004. doi: 10.1103/PhysRevLett.93.142002. URL http://link.aps.org/doi/10.1103/ PhysRevLett.93.142002.

[9] L. Berthier, G. Biroli, J.-P. Bouchaud, L. Cipelletti, and W. van Sarloos. Dynamical Heterogeneities in Glasses, Colloids and Granular Media. Oxford Science Publications, 2011.

[10] Giulio Biroli, Corinna Kollath, and Andreas M. Läuchli. Effect of rare fluctuations on the thermalization of isolated quantum systems. Phys. Rev. Lett., 105:250401, Dec 2010. doi: 10.1103/PhysRevLett.105.250401. URL http://link.aps.org/doi/10.1103/PhysRevLett. 105.250401.

[11] I. Bloch, J. Dalibard, and W. Zwerger. Many-body physics with ultracold gases. Rev. Mod. Phys., 80:885, 2008. 
[12] H.-P. Breuer and F. Petruccione. The Theory of Open Quantum Systems. Oxford University Press, 2002.

[13] C. K. Burrell and T. J. Osborne. Bounds on information propagation in disordered quantum spin chains. Phys. Rev. Lett., 99:167201, 2007.

[14] C. K. Burrell, J. Eisert, and T. J. Osborne. Information propagation through quantum chains with fluctuating disorders. Phys. Rev. A, 80:052319, 2009.

[15] P. Calabrese and J. Cardy. Evolution of entanglement entropy in one-dimensional systems. J. Stat. Mech.: Theor. Exp., page P04010, 2005.

[16] P Calabrese and J. Cardy. Time dependence of correlation functions following a quantum quench. Phys. Rev. Lett., 96:136801, 2006. doi: 10.1103/PhysRevLett.96.136801.

[17] P. Calabrese and J. Cardy. Quantum quenches in extended systems. J. Stat. Mech.: Theor. Exp., page P06008, 2007.

[18] P. Calabrese, F. H. L. Essler, and M. Fagotti. Quantum quench in the transverse-field ising chain. Phys. Rev. Lett., 106:227203, 2011.

[19] E. Calzetta and B.-L. B. Hu. Nonequilibrium Quantum Field Theory. Cambridge University Press, 2008.

[20] G. Carleo, F. Becca, M. Schiró, and M. Fabrizio. Localization and glassy dynamics of many-body quantum systems. Scientific Reports, 2:243, 2012.

[21] Giuseppe Carleo, Federico Becca, Marco Schiró, and Michele Fabrizio. Localization and glassy dynamics of many-body quantum systems. Scientific Reports, 2, 2012.

[22] Giuseppe Carleo, Federico Becca, Laurent Sanchez-Palencia, Sandro Sorella, and Michele Fabrizio. Light-cone effect and supersonic correlations in one- and two-dimensional bosonic superfluids. Phys. Rev. A, 89:031602, Mar 2014. doi: 10.1103/PhysRevA.89. 031602. URL http://link.aps.org/doi/10.1103/PhysRevA.89.031602.

[23] Amy Cassidy, Douglas Mason, Vanja Dunjko, and Maxim Olshanii. Threshold for chaos and thermalization in the one-dimensional mean-field bose-hubbard model. Phys. Rev. Lett., 105:025302, 2009.

[24] T. Castellani and A. Cavagna. Spin-glass theory for pedestrians. J. Stat. Mech.: Theor. Exp., 2005.

[25] A. H. Castro Neto and E. H. Fradkin. Bosonization of fermi liquids. Phys. Rev. B, 49: 10877, 1994. doi: 10.1103/PhysRevB.49.10877.

[26] A. H. Castro Neto and E. H. Fradkin. Exact solution of the low energy excitations of fermi liquids. Phys. Rev. Lett., 72:1393, 1994. doi: 10.1103/PhysRevLett.72.1393. 
[27] A. H. Castro Neto and E. H. Fradkin. Exact solution of the landau fixed point via bosonization. Phys. Rev. B, 51:4084, 1995. doi: 10.1103/PhysRevB.51.4084.

[28] J.-S. Caux and J. Mossel. Remarks on the notion of quantum integrability. J. Stat. Mech.: Theor. Exp., 2011.

[29] A. Cavagna. Supercooled liquids for pedestrians. Phys. Rep., 476:51, 2009.

[30] M. A. Cazalilla. Effect of suddenly turning on interactions in the luttinger model. Phys. Rev. Lett., 97:156403, 2006. doi: 10.1103/PhysRevLett.97.156403.

[31] M. A. Cazalilla, A. Iucci, and M.-C. Chung. Thermalization and quantum correlations in exactly solvable models. Phys. Rev. E, 85:011133, 2012. doi: 10.1103/PhysRevE.85.011133.

[32] D. Chandler and J. P. Garrahan. Dynamics on the way to forming glass: Bubbles in space-time. Annu. Rev. Phys. Chem., 61:191, 2010.

[33] M. Cheneau, P. Barmettler, D. Poletti, M. Endres, Peter S., T. Fukuhara, C. Gross, I. Bloch, C. Kollath, and S. Kuhr. Light-cone-like spreading of correlations in a quantum manybody system. Nature (London), 481:484, 2012.

[34] A. V. Chibukov and D. V. Khveshchenko. The effect of fermi surface curvature on lowenergy properties of fermions with singular interactions. Phys. Rev. Lett., 97:226403, 2006.

[35] Jayson G Cosme and Oleksandr Fialko. Thermalization in closed quantum systems: Semiclassical approach. Phys. Rev. A, 90(5):053602, 2014.

[36] M. Cramer, C. M. Dawson, J. Eisert, , and T. J. Osborne. Exact relaxation in a class of nonequilibrium quantum lattice systems. Phys. Rev. Lett., 100:030602, 2008.

[37] M. Cramer, A. Flesch, I. P. McCulloch, U. Schollwöck, and J. Eisert. Exploring local quantum many-body relaxation by atoms in optical superlattices. Phys. Rev. Lett., 101: 063001, 2008.

[38] J. M. Deutsch. Statistical mechanics in closed systems. Phys. Rev. A, 43:2046, 1991.

[39] Wenxin Ding, Alexander Seidel, and Kun Yang. Entanglement entropy of fermi liquids via multidimensional bosonization. Phys. Rev. X, 2:011012, Mar 2012. doi: 10.1103/ PhysRevX.2.011012. URL http://link.aps.org/doi/10.1103/PhysRevX.2.011012.

[40] M. Eckstein, M. Kollar, and P. Werner. Thermalization after an interaction quench in the hubbard model. Phys. Rev. Lett., 103:056403, 2009. doi: 10.1103/PhysRevLett.103.056403.

[41] J. Eisert and D. Gross. Supersonic quantum communication. Phys. Rev. Lett., 102:240501, 2009.

[42] L. Erdos, M. Salmhofer, and H.-T. Yau. On the quantum boltzmann equation. J. Stat. Phys., 116:367, 2004. 
[43] F. H. L. Essler, S. Kehrein, S. R. Manmana, and N. J. Robinson. Quench dynamics in a model with tuneable integrability breaking. Phys. Rev. B, 89:165104, Apr 2014. doi: 10. 1103/PhysRevB.89.165104. URL http://link.aps.org/doi/10.1103/PhysRevB.89.165104.

[44] D. Fioretto and G. Mussardo. Quantum quenches in interable field theories. 2009.

[45] H. M. Fried. Green's Functions and Ordered Exponentials. Cambridge University Press, 2002.

[46] M. Fürst, C. Mendl, and H. Spohn. Matrix-valued boltzmann equation for the nonintegrable hubbard chain. Phys. Rev. E, 88:012108, 2013.

[47] Martin L. R. Fürst, Christian B. Mendl, and Herbert Spohn. Matrix-valued boltzmann equation for the hubbard chain. Phys. Rev. E, 86:031122, Sep 2012. doi: 10.1103/ PhysRevE.86.031122. URL http://link.aps.org/doi/10.1103/PhysRevE.86.031122.

[48] J. P. Garrahan and I. Lesanovsky. Thermodynamics of quantum jump trajectories. Phys. Rev. Lett., 104:160601, 2010.

[49] J. P. Garrahan, R. L. Jack, V. Lecomte, E. Pitard, K. van Duijvendijk, and F. van Wijland. Dynamical first-order phase transition in kinetically constrained models of glasses. Phys. Rev. Lett., 98:195702, 2007.

[50] J. P. Garrahan, R. L. Jack, V. Lecomte, E. Pitard, K. van Duijvendijk, and F. van Wijland. First-order dynamical phase transition in models of glasses: an approach based on ensembles of histories. J. Phys. A: Math. Theor., 42:075007, 2009.

[51] T. Giamarchi. Quantum Physics in One Dimension. Oxford University Press, Oxford, 2004.

[52] Y. Gindikin and V. A. Sablikov. Dynamic correlations of the spinless coulomb luttinger liquid. Phys. Rev. B, 65:125109, 2002.

[53] A. Gold and A. Ghazali. Analytical results for semiconductor quantum-well wire: Plasmons, shallow impurity states, and mobility. Phys. Rev. B, 41:7626, 1990.

[54] Hermann Grabert. Projection operator techniques in nonequilibrium statistical mechanics. Springer-Verlags, 1982.

[55] M. Greiner, O. Mandel, T.W. Hänsch, and I. Bloch. Collapse and revival of the matter wave field of a bose-einstein condensate. Nature (London), 419:51, 2002.

[56] M. Gring, M. Kuhnert, T. Langen, T. Kitagawa, B. Rauer, M. Schreitl, I. Mazets, D. A. Smith, E. Demler, and J. Schmiedmayer. Relaxation and pre-thermalization in an isolated quantum system. Science, 337:1318, 2012. URL 10.1126/science.1224953.

[57] S. Habib, Y. Kluger, E. Mottola, and J. P. Paz. Dissipation and decoherence in mean field theory. Phys. Rev. Lett., 76:4660, 1996. 
[58] F. D. M. Haldane. Effective harmonic-fluid approach to low-energy properties of onedimensional quantum fluids. Phys. Rev. Lett., 47:1840, 1981.

[59] F. D. M. Haldane. 'luttinger liquid theory' of one-dimensional quantum fluids: I. properties of the luttinger model and their extension to the general $1 \mathrm{~d}$ interacting spinless fermi gas. J. Phys. C: Solid State Phys., 14:2585, 1981.

[60] Simone A. Hamerla and Götz S. Uhrig. One-dimensional fermionic systems after interaction quenches and their description by bosonic field theories. New J. Phys., 15(7): 073012, 2013. URL http://stacks.iop.org/1367-2630/15/i=7/a=073012.

[61] Simone A. Hamerla and Götz S. Uhrig. Interaction quenches in the two-dimensional fermionic hubbard model. Phys. Rev. B, 89:104301, Mar 2014. doi: 10.1103/PhysRevB.89. 104301. URL http://link.aps.org/doi/10.1103/PhysRevB.89.104301.

[62] Lester O Hedges, Robert L Jack, Juan P Garrahan, and David Chandler. Dynamic orderdisorder in atomistic models of structural glass formers. Science, 323(5919):1309-1313, 2009.

[63] A. Houghton and J. B. Marston. Bosonization and fermion liquids in dimension greater than one. Phys. Rev. B, 48:7790, 1993. doi: 10.1103/PhysRevB.48.7790.

[64] A. Houghton, H.-J. Kwon, and J. B. Marston. Stability and single-particle properties of bosonizaed fermi liquids. Phys. Rev. B, 50:1351, 1994. doi: 10.1103/PhysRevB.50.1351.

[65] A. Houghton, H.-J. Kwon, and J. B. Marston. Multidimensional bosonization. Adv. Phys., 149:141, 2000. doi: 10.1080/000187300243363.

[66] Chen-Lung Hung, Xibo Zhang, Nathan Gemelke, and Cheng Chin. Slow mass transport and statistical evolution of an atomic gas across the superfluid-mott-insulator transition. Phys. Rev. Lett., 104:160403, 2010.

[67] A. Iucci and M. A. Cazalilla. Quantum quench dynamics of the luttinger model. Phys. Rev. A, 80:063619, 2009. doi: 10.1103/PhysRevA.80.063619.

[68] A. Iucci and M. A. Cazalilla. Quantum quench dynamics of the sine-gordon model in some solvable limits. New J. Phys., 12:055019, 2010.

[69] A. Iucci and C. Naón. Exact electronic green functions in a luttinger liquid with longrange interactions. Phys. Rev. B, 61:15530, 2000.

[70] E. T. Jaynes. Information theory and statistical mechanics. Phys. Rev., 106:620, 1957.

[71] E. T. Jaynes. Information theory and statistical mechanics ii. Phys. Rev., 108:171, 1957.

[72] G. Jona-Lasinio and C. Presilla. Chaotic properties of quantum many-body systems in the thermodynamic limit. Phys. Rev. Lett., 77:4322, 1996. 
[73] Giovanni Jona-Lasinio and Carlo Presilla. Chaotic properties of quantum many-body systems in the thermodynamic limit. Phys. Rev. Lett., 77(21):4322, 1996.

[74] C. Karrasch, J. Rentrop, D. Schuricht, and V. Meden. Luttinger liquid universality in the time evolution after an interaction quench. Phys. Rev. Lett., 109:126406, 2012. doi: karrasch12_ll_universality_quench.

[75] K. Kawasaki and J. gunton. Theory of nonlinear transport processe: Nonlinear shear viscosity and normal stress effects. Phys. Rev. A, 8:2048, 1973.

[76] T. Kinoshita, T. Wenger, and D. S. Weiss. A quantum newton's cradle. Nature (London), 440:900, 2006. doi: 10.1038/nature04693.

[77] T. Koide and M. Maruyama. A new expansion of the heisenberg equation of motion with projection operator. Prog. Theor. Phys., 104:575, 2000.

[78] M. Kollar, F. A. Wolf, and M. Eckstein. Generalized gibbs ensemble prediction of prethermalization plateaus and their relation to nonthermal steady states in integrable systems. Phys. Rev. B, 84:054304, 2011. doi: 10.1103/PhysRevB.84.054304.

[79] C. Kollath, A. M. Läuchli, and E. Altman. Quench dynamics and non equilibrium phase diagram of the bose-hubbard model. Phys. Rev. Lett., 98:180601, 2007.

[80] P. Kopietz. Bosonization of Interacting Fermions in Arbitary Dimensions. Springer, 1997.

[81] R. Kubo, M. Toda, and N. Hashitsune. Statistical Physics II: Nonequilibrium Statistical Mechanics. Springer-Verlag, 1985.

[82] A. Laeuchli and C. Kollath. Spreading of correlations and entanglement after a quench in the one-dimensional bose-hubbard model. J. Stat. Mech.: Theor. Exp., page P05018, 2008 .

[83] I. Salazar Landea and N. Nessi. Prethermalization and glassiness in the bosonic hubbard model. unpublished, 2015.

[84] Tim Langen, Sebastian Erne, Remi Geiger, Bernhard Rauer, Thomas Schweigler, Maximilian Kuhnert, Wolfgang Rohringer, Igor E. Mazets, Thomas Gasenzer, and Jörg Schmiedmayer. Experimental observation of a generalized gibbs ensemble. unpublished, 2015.

[85] E. H. Lieb and D. W. Robinson. The finite group velocity of quantum spin systems. Commun. Math. Phys., 28:251, 1972.

[86] P. Linz. Analytical and numerical methods for Volterra equations. SIAM, 1985.

[87] Mingwu Lu, Nathaniel Q. Burdick, and Benjamin L. Lev. Quantum degenerate dipolar fermi gas. Phys. Rev. Lett., 108:215301, May 2012. doi: 10.1103/PhysRevLett.108.215301. URL http://link.aps.org/doi/10.1103/PhysRevLett.108.215301. 
[88] J. M. Luttinger. An exact soluble model of a many-fermion system. J. Math. Phys., 4: 1154, 1963.

[89] J. Lux, J. Muller, A. Mitra, and A. Rosch. Hydrodynamic long-time tails after a quantum quench. Phys. Rev. A, 89:053608, 2014. doi: 10.1103/PhysRevA.89.053608.

[90] Jamir Marino and Alessandro Silva. Relaxation, prethermalization, and diffusion in a noisy quantum ising chain. Phys. Rev. B, 86:060408, Aug 2012. doi: 10.1103/PhysRevB. 86.060408. URL http://link.aps.org/doi/10.1103/PhysRevB.86.060408.

[91] Jamir Marino and Alessandro Silva. Nonequilibrium dynamics of a noisy quantum ising chain: Statistics of work and prethermalization after a sudden quench of the transverse field. Phys. Rev. B, 89:024303, Jan 2014. doi: 10.1103/PhysRevB.89.024303. URL http://link.aps.org/doi/10.1103/PhysRevB.89.024303.

[92] D. C. Mattis and E. H. Lieb. Exact solution of a many-fermion system and its associated boson field. J. Math. Phys., 6:304, 1965.

[93] David McKay, Ushnish Ray, Stefan Natu, Philip Russ, David Ceperley, , and Brian DeMarco. Metastable bose-einstein condensation in a strongly correlated optical lattice. Phys. Rev. Lett., 91:023625, 2015.

[94] Aditi Mitra. Correlation functions in the prethermalized regime after a quantum quench of a spin chain. Phys. Rev. B, 87:205109, May 2013. doi: 10.1103/PhysRevB.87.205109. URL http://link.aps.org/doi/10.1103/PhysRevB.87.205109.

[95] M. Moeckel and S. Kehrein. Interaction quench in the hubbard model. Phys. Rev. Lett., 100:175702, 2008. doi: 10.1103/PhysRevLett.100.175702.

[96] M. Moeckel and S. Kehrein. Real-time evolution for weak interaction quenches in quantum systems. Ann. Phys. (N. Y.), 324:2146, 2009. doi: 10.1016/j.aop.2009.03.009. URL http://www.sciencedirect.com/science/article/pii/S0003491609000712.

[97] Michael Moeckel and Stefan Kehrein. Crossover from adiabatic to sudden interaction quenches in the hubbard model: prethermalization and non-equilibrium dynamics. New Journal of Physics, 12(5):055016, 2010. URL http://stacks.iop.org/1367-2630/12/i= $5 / \mathrm{a}=055016$.

[98] G. Mussardo. Infinite-time average of local fields in an integrable quantum field theory after a quantum quench. Phys. Rev. Lett., 111:100401, 2013.

[99] Stefan S. Natu, Kaden R. A. Hazzard, and Erich J. Mueller. Local versus global equilibration near the bosonic mott-insulator-superfluid transition. Phys. Rev. Lett., 106:125301, 2011.

[100] J. W. Negele and H. Orland. Quantum Many-particle Systems. Westview Press, 1998. 
[101] N. Nessi and A. Iucci. Quantum quench dynamics of the coulomb luttinger model. Phys. Rev. B, 87:085137, Feb 2013. doi: 10.1103/PhysRevB.87.085137. URL http://link.aps.org/ doi/10.1103/PhysRevB.87.085137.

[102] N. Nessi and A. Iucci. Equations of motion for the out-of-equilibrium dynamics of isolated quantum systems from the projection operator technique. Jour. Phys.: Conf. Ser., 568:012013, 2014.

[103] N. Nessi and A. Iucci. Glass-like behavior in a system of one dimensional fermions after a quantum quench. unpublished, 2015.

[104] N. Nessi, A. Iucci, and M. A. Cazalilla. Quantum quench and prethermalization dynamics in a two-dimensional fermi gas with long-range interactions. prl, page 210402, 2014.

[105] Zohar Nussinov. Viewpoint on the "theory of the superglass phase" and a proof of principle of quantum critical jamming and related phases. arXiv preprint arXiv:1203.4648, 2012.

[106] Zohar Nussinov, Patrick Johnson, Matthias J Graf, and Alexander V Balatsky. A dynamical relation between dual finite temperature classical and zero temperature quantum systems: quantum critical jamming and quantum dynamical heterogeneities. arXiv preprint arXiv:1209.3823, 2012.

[107] M. K. Olsen and A. S. Bradley. Numerical representation of quantum states in the positive-p and wigner representations. Optics Communications, 282:3924, 2009.

[108] Milosz Panfil, Jacopo De Nardis, and Jean-Sébastien Caux. Metastable criticality and the super tonks-girardeau gas. 2012.

[109] C Pineda, T Prosen, and E Villaseñor. Two dimensional kicked quantum ising model: dynamical phase transitions. New Journal of Physics, 16(12):123044, 2014. URL http: //stacks.iop.org/1367-2630/16/i=12/a=123044.

[110] Dario Poletti, Peter Barmettler, Antoine Georges, and Corinna Kollath. Emergence of glass-like dynamics for dissipative and strongly interacting bosons. Phys. Rev. Lett., 111: 195301, 2013.

[111] A. Polkovnikov, K. Sengupta, A. Silva, and M. Vengalattore. Nonequilibrium dynamics of closed interacting quantum systems. Rev. Mod. Phys., 83:863, 2011. doi: 10.1103/ RevModPhys.83.863.

[112] Anatoli Polkovnikov. Evolution of the macroscopically entangled states in optical lattices. Phys. Rev. A, 68(3):033609, 2003.

[113] Anatoli Polkovnikov. Phase space representation of quantum dynamics. Annals of Physics, 325(8):1790-1852, 2010. 
[114] Anatoli Polkovnikov, Subir Sachdev, and S. M. Girvin. Non-equilibrium gross-pitaevskii dynamics of boson lattice models. Phys. Rev. A, 66:053607, 2002.

[115] Sandu Popescu, Anthony J. Short, and Andreas Winter. Entanglement and the foundations of statistical mechanics. Nat. Phys., 2:754, 2006.

[116] Ilya Prigogina and Isabelle Stengers. Order Out of Chaos: Man's New Dialogue with Nature. Bentam Books, 1984.

[117] T. Prosen. Time evolution of a quantum many-body system: Transition from integrability to ergodicity in the thermodynamic limit. Phys. Rev. Lett., 80:1808, 1998.

[118] T. Prosen. Ergodic properties of a generic nonintegrable quantum many-body system in the thermodynamic limit. Phys. Rev. E, 60:3949, 1999.

[119] J. Rau and B. Müller. From reversible quantum microdynamics to irreversible quantum transport. Phys. Rep., 272:1, 1996.

[120] J. Rentrop, D. Schuricht, and V. Meden. Quench dynamics of the tomonaga-luttinger model with momentum dependent interaction. New J. Phys., 14:075001, 2012. doi: 10.1088/1367-2630/14/7/075001.

[121] M. Rigol. Breakdown of thermalization in finite one-dimensional systems. Phys. Rev. Lett., 103:100403, 2009.

[122] M. Rigol. Quantum quenches in the thermodynamic limit. Phys. Rev. Lett., 112:170601, 2014.

[123] M. Rigol and M. Fitzpatrick. Initial-state dependence of the quench dynamics in integrable quantum systems. Phys. Rev. A, 84:033640, 2011.

[124] M. Rigol, V. Dunjko, V. Yurovsky, and M. Olshanii. Relaxation in a completely integrable many-body quantum system: an ab initio study of the dynamics of the highly excited states of 1d lattice dard-core bosons. Phys. Rev. Lett., 98:050405, 2007. doi: 10.1103/ PhysRevLett.98.050405.

[125] M. Rigol, V. Dunjko, and M. Olshanii. Thermalization and its mechanism for generic isolated quantum systems. Nature (London), 452:854, 2008. doi: doi:10.1038/nature06838.

[126] B. Robertson. Equations of motion in non-equilibrium statistical mechanics. Phys. Rev., 144:151, 1966.

[127] G. Roux. Quenches in quantum many-body systems: One-dimensional bose-hubbard model reexamined. Phys. Rev. A, 79:021608, 2009.

[128] D. Ruelle. Statistical Mechanics. W. A. Benjamin, Inc., 1969.

[129] L. F. Santos and M. Rigol. Onset of quantum chaos in one-dimensional bosonic and fermionic systems and its relation to thermalization. Phys. Rev. E, 81:036206, 2010. 
[130] M. Schiró and M. Fabrizio. Time-dependent mean field theory for quench dynamics in correlated electron systems. Phys. Rev. Lett., 105:076401, 2010.

[131] H. J. Schulz. Wigner crystal in one dimension. Phys. Rev. Lett., 71:1864, 1993.

[132] R. Shankar. Renormalization-group approach to interacting fermions. Rev. Mod. Phys., 66:129, 1994.

[133] S. Sorg, L. Vidmar, L. Pollet, and F. Heidrich-Meisner. Relaxation and thermalization in the one-dimensional bose-hubbard model: A case study for the interaction quantum quench from the atomic limit. Phys. Rev. A, 90:033606, 2014.

[134] M. Srednicki. Chaos and quantum thermalization. Phys. Rev. E, 50:888, 1994.

[135] M. Stark and M. Kollar. Kinetic description of thermalization dynamics in weakly interacting quantum systems. URL http://arxiv.org/abs/1308.1610.

[136] M. Tavora and A. Mitra. Quench dynamics of one-dimensional bosons in a commensurate periodic potential: A quantum kinetic equation approach. Phys. Rev. B, 88:115144, 2013.

[137] S. Tomonaga. Remark on bloch's method of sound waves applied to many-fermion problesm. Prog. Theor. Phys., 5:544, 1950.

[138] S. Trotzky, Y.-A. Chen, A. Flesch, I. P. McCulloch, U. Schollwã ๆck, J. Eisert, and I. Bloch. Probing the relaxation towards equilibrium in an isolated strongly correlated 1d bose gas. Nat. Phys., 8:325, 2012.

[139] S. Trotzky, Y.-A. Chen, A. Flesch, I. P. McCulloch, U. Schollwöck, J. Eisert, and I. Bloch. Probing the relaxation towards equilibrium in an isolated strongly correlated $1 \mathrm{~d}$ bose gas. Nat. Phys., 8:325, 2012.

[140] M. van den Worm, B.C. Sawyer, J.J. Bollinger, and M. Kastner. Relaxation timescales and decay of correlations in a long-range interacting quantum simulator. New J. of Phys, 15: 83007, 2013.

[141] D. W. Wang, A. J. Millis, and S. Das Sarma. Coulom luttinger liquid. Phys. Rev. B, 64: 193307, 2001.

[142] B. Wouters, J. De Nardis, M. Brockmann, D. Fioretto, M. Rigol, and J.-S. Caux. Quenching the anisotropic heisenberg chain: Exact solution and generalized gibbs ensemble predictions. Phys. Rev. Lett., 113:117202, 2014.

[143] T.-Y. Wu. Kinetic Equations of Gases and Plasmas. Addison-Wesley, 1966.

[144] Pablo R. Zangara, Axel D. Dente, E. J. Torres-Herrera, Horacio M. Pastawski, Aníbal Iucci, and Lea F. Santos. Time fluctuations in isolated quantum systems of interacting particles. Phys. Rev. E, 88:032913, Sep 2013. doi: 10.1103/PhysRevE.88.032913. URL http://link.aps.org/doi/10.1103/PhysRevE.88.032913. 